\title{
GAS-PHASE BASICITIES OF POLYFUNCTIONAL MOLECULES. PART 1: THEORY AND METHODS
}

\author{
Guy Bouchoux* \\ Laboratoire des Mécanismes Réactionnels, Département de Chimie, Ecole \\ Polytechnique, 91120 Palaiseau, France
}

Received 11 January 2007; accepted 18 June 2007

Published online 13 September 2007 in Wiley InterScience (www.interscience.wiley.com) DOI 10.1002/mas.20151

\begin{abstract}
The experimental and theoretical methods of determination of gas-phase basicities, proton affinities and protonation entropies are presented in a tutorial form. Particularities and limitations of these methods when applied to polyfunctional molecules are emphasized. Structural effects during the protonation process in the gas-phase and their consequences on the corresponding thermochemistry are reviewed and classified. The role of the nature of the basic site (protonation on non-bonded electron pairs or on $\pi$-electron systems) and of substituent effects (electrostatic and resonance) are first examined. Then, linear correlations observed between gas-phase basicities and ionization energies or substituent constants are recalled. Hydrogen bonding plays a special part in proton transfer reactions and in the protonation characteristics of polyfunctional molecules. A survey of the main properties of intermolecular and intramolecular hydrogen bonding in both neutral and protonated species is proposed. Consequences on the protonation thermochemistry, particularly of polyfunctional molecules are discussed. Finally, chemical reactions which may potentially occur inside protonated clusters during the measurement of gas-phase basicities or inside a protonated polyfunctional molecule is examined. Examples of bond dissociations with hydride or alkyl migrations, proton transport catalysis, tautomerization, cyclization, ring opening and nucleophilic substitution are presented to illustrate the potentially complex chemistry that may accompany the protonation of polyfunctional molecules. (C) 2007 Wiley Periodicals, Inc., Mass Spec Rev 26:775-835, 2007
\end{abstract}

Keywords: gas-phase basicity; proton affinity; protonation entropy; polyfunctional molecules; thermochemistry; hydrogen bond; substituent effects

\section{INTRODUCTION}

The protonic definition of acidity and basicity (Brönsted, 1928) is one of the founding concepts of modern chemistry which still motive active interest and researches in the scientific community. Accordingly, protonation reactions are ubiquitous events in almost all the areas of chemistry and biochemistry. As underlined more than 30 years ago by Bell (1973), proton is the simplest monocharged cation, it is unique in having no electron outside the

*Correspondence to: Guy Bouchoux, Laboratoire des Mécanismes Réactionnels, UMR CNRS 7651, Ecole Polytechnique, 91128 Palaiseau Cedex, France. E-mail: bouchoux@dcmr.polytechnique.fr nucleus and thus the smallest radius as compared to all other ions $\left(10^{-15} \mathrm{~m}\right.$ against $\left.\sim 10^{-10} \mathrm{~m}\right)$. One consequence of this characteristic is a considerable polarizing power on neighbor atoms or molecular entities. This phenomenon is, in particular, at the origin of considerable effects in the strengths of the bonds located in the vicinity of a protonated center, of the existence of hydrogen bonds and of the easiness of proton transfer.

The structural and energetic changes induced by the polarizing power of the proton are expected to be at its maximum when an isolated, non-solvated, species is considered. Thus, the thermochemical concept of gas-phase basicity is certainly the ideal revelator of the structural and energetic characteristics of a single, molecular, protonation process. This remark takes more weight in the case of polyfunctional compounds since, in the absence of solvent, a direct interaction between the different functional groups is allowed to occur, both in the molecule and its protonated form. It is frequently underlined that mass spectrometry, by its capability to handle individual ions, allows the determination of intrinsic molecular properties, separated from solvation effects. Indeed, since the 1980s, the gas-phase basicity of a large variety of organic molecules has been measured. The results of these measurements were integrated in an evaluated tabulation of over 2,000 compounds in 1998 (Hunter \& Lias, 1998) and new data continue to increase the gas-phase thermochemical patrimony. Historically, the first measurements were concerned with simple monofunctional or bifunctional compounds. With the advent of new ionization techniques based on protonation processes, the analysis by mass spectrometry has been extended to larger molecules, mostly polyfunctional. To interpret conveniently the dissociation of the protonated forms of these large molecules, the knowledge of the sites of proton attachment and of the corresponding enthalpic and entropic changes is of considerable interest. Determination of the gasphase basicities of polyfunctional molecules remains (or becomes) consequently an actual question. It is however a challenging task since several difficulties are attached to the nature of these compounds. One crucial point is that these molecules are largely non-volatile and even thermolabile. This peculiarity limits the experimental methods of determination of the gas-phase basicity to the kinetic or the thermokinetic methods. By definition large molecules contain a large number of atoms which and limits the computational modelization of the protonation process by the current quantum chemical procedures. A large number of atoms means also frequently a large number of conformers for the neutral and the protonated species thus complicating the molecular interpretation of the experimental data. Finally, interfunctional interactions may induce structural 
constraints or changes in both the neutral and the protonated forms of the base. These phenomena are at the origin of entropy loss or gain during protonation and sometimes to "internal chemistry" involving covalent bond forming or bond breaking.

The present review will present a general overview of the methods used to determine the protonation thermochemistry of gaseous species together with the underlying theory. Emphasis will be given to the validity of the hypothesis and methods when polyfunctional molecules are considered. The second part will recall the various structural changes induced by the protonation of isolated molecules and the thermochemical consequences. The effects of the nature of the basic site, of the substituents, of intra- or inter-molecular hydrogen bonding and of possible interfunctional chemical reactions on the proton affinity and the protonation entropy are presented. Other sections are planned which will be devoted to homologous series of organic and bio-organic molecules: aliphatic, aromatic, aminoacids and peptides...

\section{BACKGROUND}

\section{A. General}

\section{Definitions of Thermodynamic Quantities}

Ion thermochemical data are usually defined as differences in the relevant thermodynamic function between products and reactants of a specified formal reaction. To handle positive molar enthalpy differences, the considered reactions are always taken in the endothermic direction, that is, generally for a dissociative pathway. In line with this general rule, the thermochemical quantities associated with the concept of gas-phase basicity of a given molecule $\mathrm{M}$ are defined by considering the deprotonation reaction of the $\mathrm{MH}^{+}$ion (1):

$$
\begin{aligned}
& \underset{(\text { gas })}{\mathrm{MH}^{+}} \rightarrow \underset{(\text { gas })}{\mathrm{M}}+\underset{(\text { gas })}{\mathrm{H}^{+}} \\
& \text {Proton affinity, } \mathrm{PA}(\mathrm{M})=\Delta_{1} H^{0} \\
& \text { Gas phase basicity, } \mathrm{GB}(\mathrm{M})=\Delta_{1} G^{0} \\
& \text { Protonation entropy, } \Delta_{p} S^{0}(\mathrm{M})=S^{0}\left(\mathrm{MH}^{+}\right)-S^{0}(\mathrm{M})
\end{aligned}
$$

Proton affinity, PA(M), and gas-phase basicity, GB(M), of the molecule $\mathrm{M}$ are the standard enthalpy and standard Gibbs free energy of reaction (1), respectively. Note thermochemical compilations continue to use a reference pressure of $1 \mathrm{~atm}$. in spite of the IUPAC recommendation which proposes to use 1 bar, that is, $0.987 \mathrm{~atm}$. Proton affinity and gas-phase basicity may be determined at any temperature $\mathrm{T}$ but the tabulated values (Lias, Liebman, \& Levin, 1984; Lias et al., 1988; Hunter \& Lias, 1998, 2005; Lias \& Bartmess, 2000) are generally given for the room temperature of $298.15 \mathrm{~K}$.

Introducing the entropy of the reaction (1), $\Delta_{1} S_{T}^{0}$, the thermodynamic relationship $\Delta G_{T}^{0}=\Delta H_{T}^{0}-T \Delta S_{T}^{0}$ obviously leads to Equation (2) at $298 \mathrm{~K}$ :

$$
\mathrm{GB}_{298}(\mathrm{M})=\mathrm{PA}_{298}(\mathrm{M})-298 \Delta_{1} S_{298}^{0}
$$

Proton affinity, $\mathrm{PA}_{298}(\mathrm{M})$ (i.e., $\Delta_{1} H_{298}^{0}$ ) can be also expressed in terms of individual heats of formation of the species involved in reaction (1):

$$
\mathrm{PA}_{298}(\mathrm{M})=\Delta_{1} H_{298}^{0}=\Delta_{\mathrm{f}} H_{298}^{0}(\mathrm{M})+\Delta_{\mathrm{f}} H_{298}^{0}\left(\mathrm{H}^{+}\right)-\Delta_{\mathrm{f}} H_{298}^{0}\left(\mathrm{MH}^{+}\right)
$$

The $298 \mathrm{~K}$ heat of formation of the isolated proton, $\Delta_{\mathrm{f}} H_{298}^{0}\left(\mathrm{H}^{+}\right)$, appearing in Equation 3 is a well-known quantity, equal to $1,530 \mathrm{~kJ} \mathrm{~mol}^{-1}$ using the so-called "ion convention". Two conventions have been adopted for the treatment of the electron which leads to slightly different heat of formation values of charged species. In the "electron convention", the heat of formation of the electron, $\Delta_{\mathrm{f}} H_{T}^{0}\left(\mathrm{e}^{-}\right)$, is taken as zero at all temperatures, while in the "ion convention" $\Delta_{\mathrm{f}} H_{T}^{0}\left(\mathrm{e}^{-}\right)$, is defined as its integrated heat capacity at temperature $T$, that is, $5 / 2 R T$ in the classical Boltzmann statistics (see Bartmess, 1994). The ion convention has been adopted by all the ion thermochemistry compilations (Lias, Liebman, \& Levin, 1984; Lias et al., 1988; Hunter \& Lias, 1998, 2005; Lias \& Bartmess, 2000) and will be used here along this review.

The entropy of reaction (1), $\Delta_{1} S_{298}^{0}$, is equal to the difference in absolute entropies of the products and reactant, Equation (4):

$$
\Delta_{1} S_{298}^{0}=S_{298}^{0}(\mathrm{M})+S_{298}^{0}\left(\mathrm{H}^{+}\right)-S_{298}^{0}\left(\mathrm{MH}^{+}\right)
$$

It is a custom to define the "protonation entropy" or "half reaction entropy" for a species $\mathrm{M}$, at temperature $T$, by the difference in absolute entropies:

$$
\Delta_{\mathrm{p}} S_{T}^{0}(\mathrm{M})=S_{T}^{0}\left(\mathrm{MH}^{+}\right)-S_{T}^{0}(\mathrm{M})
$$

and to consequently write for the entropy of reaction (1):

$$
\Delta_{1} S_{T}^{0}=S_{T}^{0}\left(\mathrm{H}^{+}\right)-\Delta_{\mathrm{p}} S_{T}^{0}(\mathrm{M})
$$

These notations allow expressing the gas-phase basicity as

$$
\mathrm{GB}_{298}(\mathrm{M})=\mathrm{PA}_{298}(\mathrm{M})-298\left[S_{298}^{0}\left(\mathrm{H}^{+}\right)-\Delta_{\mathrm{p}} S_{298}^{0}(\mathrm{M})\right]
$$

The translational entropy of $1 \mathrm{~mol}$ of protons, considered as an ideal monoatomic gas, at a temperature $T$ may be estimated from statistical thermodynamic using the Sackur-Tetrode equation (Eq. (23) thereafter) to $S_{T}^{0}\left(\mathrm{H}^{+}\right)=20.786 \ln (T)-$ $9.685 \mathrm{~J} \mathrm{~mol}^{-1} \mathrm{~K}^{-1}$. At $298 \mathrm{~K}$, this relationship gives $S_{298}^{0}\left(\mathrm{H}^{+}\right)=108.7 \mathrm{~J} \mathrm{~mol}^{-1} \mathrm{~K}^{-1}$ and consequently the following equality holds:

$$
\mathrm{GB}_{298}(\mathrm{M})=\mathrm{PA}_{298}(\mathrm{M})-298 \Delta_{\mathrm{p}} S_{298}^{0}(\mathrm{M})-32.4 \mathrm{~kJ} \mathrm{~mol}^{-1}
$$

A graphical view of the interrelationship between proton affinity, PA(M), and gas-phase basicity, GB(M) (at $298 \mathrm{~K}$ ), is given in Scheme 1.

As a consequence or their definitions from deprotonation reaction (1), proton affinity and gas-phase basicity are positive quantities. Their values are roughly situated in the 600$1,100 \mathrm{~kJ} \mathrm{~mol}^{-1}$ range for organic molecules (see Gas-Phase Basicity Scale). It is noteworthy that PA (M) and GB (M) increase with increasing the basic strength and are therefore direct measures of the intrinsic basicity of the molecule M. Protonation entropy, $\Delta_{\mathrm{p}} S_{T}^{0}(\mathrm{M})$, the necessary link between PA (M) and 


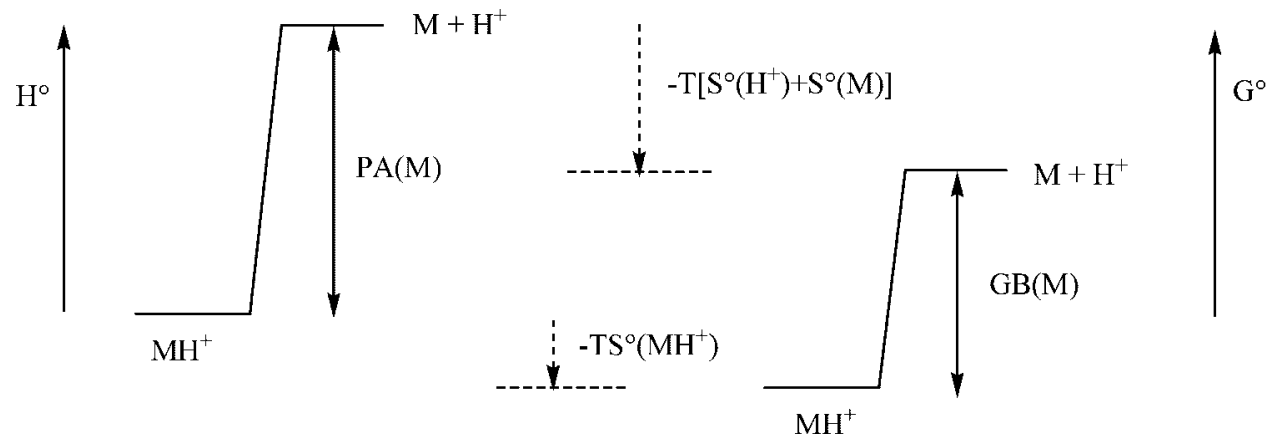

SCHEME 1.

GB (M), may be seen as a measure of the disorder appearing upon protonation of the molecule M. Structural effects and, in particular, substituents interactions in polyfunctional molecules $\mathrm{M}$, or their protonated forms $\mathrm{MH}^{+}$, have a profound influence on the $\Delta_{\mathrm{p}} S_{T}^{0}(\mathrm{M})$ value. Actually, if for a simple monofunctional molecule $\Delta_{\mathrm{p}} S_{T}^{0}(\mathrm{M})$ value rarely exceed $\pm 20 \mathrm{~J} \mathrm{~mol}^{-1} \mathrm{~K}^{-1}$, it may attain large positive or negative values (say $>50 \mathrm{~J} \mathrm{~mol}^{-1} \mathrm{~K}^{-1}$ in absolute value) for polyfunctional molecules. This point will be amply illustrated in the development of this review.

Experimental determinations of the protonation thermochemistry are generally conducted at temperature $\mathrm{T}$ different from $298 \mathrm{~K}$ and the influence of temperature on enthalpy and absolute entropy has to be considered. For reaction (1), for example, enthalpy and entropy changes with temperature are given by Equations (10) and (11):

$$
\begin{aligned}
& \Delta_{1} H_{T 2}^{0}=\Delta_{1} H_{T 1}^{0}+\int_{T 1}^{T 2} \Delta_{1} C_{p} \mathrm{~d} T \\
& \Delta_{1} S_{T 2}^{0}=\Delta_{1} S_{T 1}^{0}+\int_{T 1}^{T 2} \frac{\Delta_{1} C_{p} \mathrm{~d} T}{T}
\end{aligned}
$$

where $\Delta_{1} C_{p}$ is the difference in heat capacities $C_{p}\left(\mathrm{H}^{+}\right)+$ $C_{p}(\mathrm{M})-C_{p}\left(\mathrm{MH}^{+}\right)$. In a first approximation, $C_{p}\left(\mathrm{H}^{+}\right)=5 / 2 R$, that is, $20.8 \mathrm{~J} \mathrm{~mol}^{-1} \mathrm{~K}^{-1}$ and $C_{p}(\mathrm{M})$ is close, or even slightly less than $C_{p}\left(\mathrm{MH}^{+}\right)$. Consequently, maximum values for $\Delta_{1} H_{T 2}^{0}-\Delta_{1} H_{T 1}^{0}$ and $\Delta_{1} S_{T 2}^{0}-\Delta_{1} S_{T 1}^{0}$ are ca. $21\left(T_{2}-T_{1}\right) \mathrm{J} \mathrm{mol}^{-1}$ and $21 \ln \left(T_{2} / T_{1}\right) \mathrm{J} \mathrm{mol}^{-1} \mathrm{~K}^{-1}$. In the temperature range $298-$ $1,000 \mathrm{~K}$ these enthalpy and entropy corrections are consequently equal to, or less than, $15 \mathrm{~kJ} \mathrm{~mol}^{-1}$ and $25 \mathrm{~J} \mathrm{~mol}^{-1} \mathrm{~K}^{-1}$, respectively. These terms are clearly difficult to neglect and care must be taken when handling data at temperature (real of "effective") different from $298 \mathrm{~K}$. It will be seen however, that most of the experiments involve proton transfer reactions leading to an overall difference in heat capacities significantly reduced (see Eq. 47 to 53 and the relevant comments later on).

\section{Statistical Thermodynamic Results}

Statistical thermodynamic (McQuarrie, 1973) is the unique mean to calculate macroscopic thermochemical properties from the characteristics of the individual molecules making up the system. It is an important tool for the understanding and for the treatment of experimental data concerning protonation thermochemistry. Several essential results are given in this section, consequences related to the protonation of polyfunctional molecules will be discussed in Structural and Energetic Aspects of the Protonation.

a. The partition function and the derivation of thermochemical functions. Let us consider a system of $N$ molecules (or ions) of quantified molecular energy levels $\varepsilon_{1}, \ldots, \varepsilon_{i}, \ldots$ with respect to the fundamental $\varepsilon_{0}$ level, in thermal equilibrium at temperature $T$. The number $n_{i}$ of molecules at level $\varepsilon_{i}$ may be deduced from the Boltzmann distribution equation:

$$
\frac{n_{i}}{n_{0}}=g_{i} \exp \left(\frac{-\varepsilon_{i}}{k_{\mathrm{B}} T}\right)
$$

therefore, since $N=\sum n_{i}$, the number of molecules in level $i$ is given by

$$
\frac{n_{i}}{N}=\frac{g_{i} \exp \left(-\varepsilon_{i} / k_{\mathrm{B}} T\right)}{\sum g_{i} \exp \left(-\varepsilon_{i} / k_{\mathrm{B}} T\right)}
$$

where $g_{i}$ is the degeneracy of the $\varepsilon_{i}$ level and $k_{\mathrm{B}}$ the Boltzmann constant. The denominator of Equation (12), that is, the sum of the particle states at temperature $T$, is called the "partition function," $Q$, of the system. It is seen from the preceding equation that $Q=N / n_{0}$, thus when all the molecules are in the zero level $Q=1$ and as more molecules occupy higher levels $Q$ increases. Statistical thermodynamic uses the partition function $Q$ to calculate the thermodynamic functions $U, H, G, S, \ldots$ Calculation is usually done for $1 \mathrm{~mol}$ of an ideal gas at a pressure of 1 atm. (101,325 Pa).

The molar thermochemical functions are expressed with reference to the $0 \mathrm{~K}$ state as follows:

$$
\begin{gathered}
\text { internal energy } U_{T}^{0}=R T^{2}\left(\frac{\partial \ln Q}{\partial T}\right)_{V} \\
\text { enthalpy } H_{T}^{0}=R T^{2}\left(\frac{\partial \ln Q}{\partial T}\right)_{P} \\
\text { entropy } S_{T}^{0}=\operatorname{RT}\left(\frac{\partial \ln Q}{\partial T}\right)_{V}+R \ln Q
\end{gathered}
$$

Gibbs free energy $\quad G_{T}^{0}=H_{T}^{0}-T S_{T}^{0}$ 
heat capacities $C_{P}=\left(\frac{\partial H}{\partial T}\right)_{P}=2 R T\left(\frac{\partial \ln Q}{\partial T}\right)_{P}+R T^{2}\left(\frac{\partial^{2} \ln Q}{\partial T^{2}}\right)_{P}$

$$
C_{V}=\left(\frac{\partial U}{\partial T}\right)_{V}=2 R T\left(\frac{\partial \ln Q}{\partial T}\right)_{V}+R T^{2}\left(\frac{\partial^{2} \ln Q}{\partial T^{2}}\right)_{V}
$$

The calculation of these quantities is simplified by considering the fact that the energy of a polyatomic molecule may be separated into its electronic, translational, rotational, and vibrational components:

$$
\varepsilon_{i}=\varepsilon_{\text {elec }}+\varepsilon_{\text {transl }}+\varepsilon_{\text {rot }}+\varepsilon_{\text {vib }}
$$

Molecules and their protonated forms have generally only one electronic state with zero spin, the electronic term $\varepsilon_{\text {elec }}$ is thus zero. The three remaining terms $\varepsilon_{\text {transl }}, \varepsilon_{\text {rot }}$ and $\varepsilon_{\text {vib }}$ may be conveniently separated into the $3 n$ degrees of freedom of the molecule or ion (with $n=$ number of atoms of the molecular specie). These will be three translational, 3 (or 2 for a linear molecule or 0 for an atom) rotational as a whole and the remainder vibrational degrees of freedom. The separability of the energy terms following Equation (18), implies that the overall partition function $Q$ will be a product of electronic, translational, rotational and vibrational partition functions. Since the thermodynamic functions $U, H, S, G, \ldots$ depends on the logarithm or the logarithmic derivative of $Q$ (see Eqs. 13-17), they will be calculated as sums of contributions for the different types of molecular motions. For example molar entropy may be calculated by $S_{T}^{0}=\left(S_{T}^{0}\right)_{\text {elec }}+\left(S_{T}^{0}\right)_{\text {transl }}+\left(S_{T}^{0}\right)_{\text {rot }}+\left(S_{T}^{0}\right)_{\text {vib }}$. Explicit forms of these contributions are dependent on the nature of the degrees of freedom as it will be now detailed, with emphasis on enthalpy and entropy.

b. Translation. The translational partition function of $1 \mathrm{~mol}$ of ideal gas depends only of $P, T$ and the mass $m$ of the molecule. Its complete expression at $P=1 \mathrm{~atm}$. leads to:

$$
\begin{aligned}
& \left(U_{T}^{0}\right)_{\text {transl }}=\frac{3}{2 R T} \\
& \left(H_{T}^{0}\right)_{\text {transl }}=\frac{5}{2 R T} \\
& \left(S_{\mathrm{T}}^{0}\right)_{\text {transl }}=\frac{5}{2} R+R \ln \left[\left(\frac{2 \pi \mathrm{m}}{h^{2}}\right)^{3 / 2}\left(k_{\mathrm{B}} T\right)^{5 / 2}\right] \\
& \left(C_{P}^{0}\right)_{\text {transl }}=\frac{5}{2 R T} \\
& \left(C_{V}^{0}\right)_{\text {transl }}=\frac{3}{2 R T}
\end{aligned}
$$

Equation (21) is known as the Sackur-Tetrode equation. When $m$ is expressed in atomic mass unit $(\mathrm{u})$ the following expression gives $\left(S_{T}^{0}\right)_{\text {transl }}$ in $\mathrm{J} \mathrm{mol}^{-1} \mathrm{~K}^{-1}$ :

$$
\left(S_{T}^{0}\right)_{\text {transl }}=\left(\frac{3}{2}\right) R \ln [m]+\left(\frac{5}{2}\right) R \ln [T]-9.685
$$

(note that $R=8.314511 \mathrm{~J} \mathrm{~mol}^{-1} \mathrm{~K}^{-1}$ ).

c. Overall rotations. In the general case of a non-linear polyatomic molecule there are three "external" rotational degrees of freedom. For a rigid system, the final expression of the rotational partition function includes the temperature, $T$, the product of the three principal moments of inertia about the center of mass, $I_{\mathrm{A}} I_{\mathrm{B}} I_{\mathrm{C}}$, and the molecular symmetry number $\sigma$. This latter is defined as the total number of independent permutations of identical atoms in a molecule that can be attained by simple rigid rotations of the entire molecule. Typical examples are $\mathrm{H}_{2} \mathrm{O}$ $(\sigma=2), \mathrm{NH}_{3}(\sigma=3), \mathrm{CH}_{4}$ and benzene $(\sigma=12)$. The contributions to thermochemical function of the overall rotations of a nonlinear molecule are given by

$$
\begin{aligned}
& \left(U_{T}^{0}\right)_{\text {rot }}=\frac{3}{2 R T} \\
& \left(H_{T}^{0}\right)_{\text {rot }}=\frac{3}{2 R T} \\
& \left(S_{T}^{0}\right)_{\text {rot }}=\frac{3}{2} R+\mathrm{R} \ln \left[\frac{8 \pi^{2}\left(2 \pi k_{\mathrm{B}} T\right)^{3 / 2}\left(I_{\mathrm{A}} I_{\mathrm{B}} I_{\mathrm{C}}\right)^{1 / 2}}{\sigma h^{3}}\right] \\
& \left(C_{P}^{0}\right)_{\mathrm{rot}}=\frac{3}{2} R \\
& \left(C_{V}^{0}\right)_{\mathrm{rot}}=\frac{3}{2} R
\end{aligned}
$$

After substituting the values of the constants and expressing each moment of inertia in $\mathrm{u} \AA^{2}$, the value of $\left(S_{T}^{0}\right)_{\text {rot }}$ in $\mathrm{J} \mathrm{mol}^{-1} \mathrm{~K}^{-1}$ is obtained from:

$$
\left(S_{T}^{0}\right)_{\mathrm{rot}}=\frac{1}{2} R \ln \left(I_{\mathrm{A}} I_{\mathrm{B}} I_{\mathrm{C}}\right)+\frac{3}{2} R \ln T-R \ln \sigma-22.537
$$

d. Vibrations. A non-linear molecule with $\mathrm{n}$ atoms is characterized by $(3 n-6)$ vibrational degrees of freedom. Among these, a major part can be considered as true valence and deformation vibrations but some degrees of freedom may correspond to large amplitude motions responsible of the floppy character of the molecule. A specific treatment of the latter is particularly important in the context of the present review and will be developed below. Let us first consider a molecule showing up to $(3 n-6)$ vibrational degrees of freedom for which the harmonic oscillator approximation is valid. In this approximation, each vibration is treated as a true harmonic oscillator and is supposed to be independent of the others. The $(3 n-6)$ total partition function may be thus expressed as a product of one mode partition functions. Under these circumstances, the vibrational contributions to $U$ and $H$ are given by

$$
\left(U_{T}^{0}\right)_{\mathrm{vib}}=\left(H_{T}^{0}\right)_{\mathrm{vib}}=R T \sum_{i=1}^{3 n-6} \frac{h v_{i} / k_{\mathrm{B}} T}{\exp \left(h v_{i} / k_{\mathrm{B}} T\right)-1}
$$

to which the zero point vibrational energy, ZPVE, should be added:

$$
\mathrm{ZPVE}=\sum_{i=1}^{3 n-6} \frac{h v_{i}}{2}
$$

Similarly, the contribution to the entropy may be expressed as Equation (31):

$$
\left(S_{T}^{0}\right)_{\text {vib }}=R \sum_{i=1}^{3 n-6}\left[\frac{h v_{i} / k_{\mathrm{B}} T}{\exp \left(h v_{i} / k_{\mathrm{B}} T\right)-1}-\ln \left[1-\exp \left(\frac{-h v_{i}}{k_{\mathrm{B}} T}\right)\right]\right]
$$


which consequently leads to the Gibbs free energy term:

$$
\left(G_{T}^{0}\right)_{\mathrm{vib}}=R T \sum_{i=1}^{3 n-6} \ln \left[1-\exp \left(\frac{-h v_{i}}{k_{\mathrm{B}} T}\right)\right]
$$

The vibrational contribution to $C_{P}$ or $C_{V}$ may be deduced from Equations (17) and (29):

$$
\left(C_{P, T}^{0}\right)_{\mathrm{vib}}=\left(C_{V, T}^{0}\right)_{\mathrm{vib}}=R \sum_{i=1}^{3 n-6}\left(\frac{\left(h v_{i} / k_{\mathrm{B}} T\right)^{2} \exp \left(h v_{i} / k_{\mathrm{B}} T\right)}{\left[\exp \left(h v_{i} / k_{\mathrm{B}} T\right)-1\right]^{2}}\right)
$$

At this stage, it is interesting to visualize the role of the frequency $v_{i}$ on the $\left(H_{T}^{0}\right)_{\mathrm{vib}},\left(S_{T}^{0}\right)_{\mathrm{vib}}$ and $\left(C_{P, T}^{0}\right)_{\mathrm{vib}}$ terms. Figure 1 shows the three contributions brought by one harmonic oscillator as a function of the wave number. It readily appears from examination of Figure 1 that small frequencies contribute more than larger frequencies to these three thermochemical quantities. Note that the limiting values of $\left(H_{T}^{0}\right)_{\mathrm{vib}}$ and $\left(C_{P, T}^{0}\right)_{\mathrm{vib}}$ given by the harmonic oscillator model when $v$ tends to zero are equal to $R T$ (i.e., $2.479 \mathrm{~kJ} \mathrm{~mol}^{-1}$ at $298 \mathrm{~K}$ ) and $R\left(8.3145 \mathrm{~J} \mathrm{~mol}^{-1} \mathrm{~K}^{-1}\right)$ respectively while entropy is expected to tends to infinity. These limits are however unrealistic since low frequencies corresponds generally to degrees of freedom hardly describable by the harmonic oscillator model.

e. Internal rotations. As mentioned above, in a number of organic molecules, one or several of the $(3 n-6)$ internal degrees of freedom may present large amplitude motions where the harmonic approximation to the potential energy profile does not necessarily hold. The most widespread case of this type of situation is, without doubt, internal rotation but other anharmonic motions, such as inversion of a non-planar trivalent center, ring puckering and pseudorotation, enter also in this category. Anharmonic vibrational motion raises two problems, first a specific partition function should be introduced in place of the harmonic oscillator partition function, and, second, the coupling of this oscillator with other degrees of freedom should be considered.

If we consider the simplest case of a rotation of a symmetric group of symmetry number $n$, the potential energy function $V(\phi)$ associated with the internal rotation is given by

$$
V(\phi)=\frac{1}{2} V_{0}(1-\cos n \phi)
$$

with $\phi$ the dihedral angle and $V_{0}$ the barrier height. Change in potential energy during the rotation leads to $n$ minima corresponding to $n$ conformers of identical energy separated by $n$ maxima of height $V_{0}$. According to the available internal energy of the system with respect to $V_{0}$ three situations may be distinguished.

For energies well below the barrier, $V_{0}$, that is, when the internal energy of the molecule is not large enough to pass the potential energy barrier, each conformer is "trapped" at the bottom of the potential energy wells. The motion is that of a simple vibration which may be described by the harmonic oscillator model. Accordingly, for small values of $\phi$ Equation (34) reduces to $V(\phi)=(1 / 2) V_{0}(n \phi)^{2}$ which corresponds to the potential energy of a harmonic oscillator of fundamental frequency:

$$
v_{\text {harm.rot }}=\left(\frac{n}{2 \pi}\right)\left(\frac{V_{0}}{2 I_{\text {red }}}\right)^{1 / 2}
$$

The thermochemical functions can thus be obtained by using Equations (29)-(33) and replacing $v_{i}$ by $v_{\text {harm.rot }}$. Note that in most cases of internal rotations in organic molecules, $V_{0}$ rarely exceed $100 \mathrm{~kJ} \mathrm{~mol}^{-1}$ and the reduced moment $I_{\text {red }}$ is rarely below $3 \mathrm{u} \AA^{2}$. This means that $v_{\text {harm.rot }}$ is classically below $200 \mathrm{~cm}^{-1}$.

At the opposite, if the internal energy is far above $V_{0}$, the rotation may be considered as essentially free and the thermochemical functions can be calculated in the rigid free rotor approximation using Equations (36)-(38):

$$
\begin{aligned}
& \left(U_{T}^{0}\right)_{\text {free.rot }}=\left(H_{T}^{0}\right)_{\text {free.rot }}=\frac{1}{2 R T} \\
& \left(C_{P}^{0}\right)_{\text {free.rot }}=\left(C_{V}^{0}\right)_{\text {free.rot }}=\frac{1}{2} R \\
& \left(S_{T}^{0}\right)_{\text {free.rot }}=\frac{1}{2} R+\frac{1}{2} R \ln \left[\frac{8 \pi^{3} k_{\mathrm{B}} T I_{\text {red }}}{h^{2} n^{2}}\right]
\end{aligned}
$$

where $I_{\text {red }}$ is the reduced moment of inertia (in $\mathrm{u} \AA^{2}$ ) of the two rotating groups around the axis containing the twisting bond and $\mathrm{n}$ the symmetry of the rotation. For practical purpose, when $I_{\text {red }}$ is expressed in $\mathrm{u}^{2}$, Equation (38) reduces to Equation (39):

$$
\left(S_{T}^{0}\right)_{\text {free.rot }}=\frac{1}{2} R \ln \left[\frac{T I_{\text {red }}}{n^{2}}\right]-4.3396\left(\mathrm{in} \mathrm{J} \mathrm{mol}^{-1} \mathrm{~K}^{-1}\right)
$$

Finally, the most general hindered rotation case arises when $V_{0}$ and the internal energy of the molecule are of the same order of magnitude. To get the rotational energy levels used for calculating the hindered rotor partition function, it is necessary to solve the corresponding one-dimensional Schrödinger equation usually approximated, in the case of a single torsion, by

$$
\frac{-\hbar^{2}}{2 I_{i}} \frac{\partial^{2} \Psi_{\kappa}}{\partial \phi^{2}}+V(\phi) \Psi_{\kappa}=\varepsilon_{\kappa} \Psi_{\kappa}
$$

where $V(\phi)$ is given by Equation (34). The partition function of a hindered rotation and the corresponding thermochemical functions cannot be given by simple expressions and must be numerically calculated or approximated. More than 60 years ago, Pitzer and Gwinn (1942) proposed a treatment of internal rotation for a rigid molecule with attached symmetrical top and tabulated the resulting thermodynamic functions. Considering these results, approximate procedures have been proposed to evaluate these thermochemical contributions (Truhlar, 1991; McClurg, Flagan, \& Goddard, 1997; Witschel \& Hartwigsen, 1997; Barker \& Shovlin, 2004). Rigorous formalism of non-symmetrical torsions and the question of the moment of inertia for such internal rotations have been recently presented (Chuang \& Truhlar, 2000). Development of methods of evaluation of the internal rotational entropy of molecules containing several internal rotational degrees of freedom by means of Fourier expansion of the potential energy profiles $V\left(\phi_{1}\right)$ obtained from molecular orbital or molecular dynamic calculations have been recently developed (Van Speybroeck et al., 2000, 2001, 2005; Van Speybroeck, Van Neck, \& Waroquier, 2002; Zhu \& Bozzelli, 

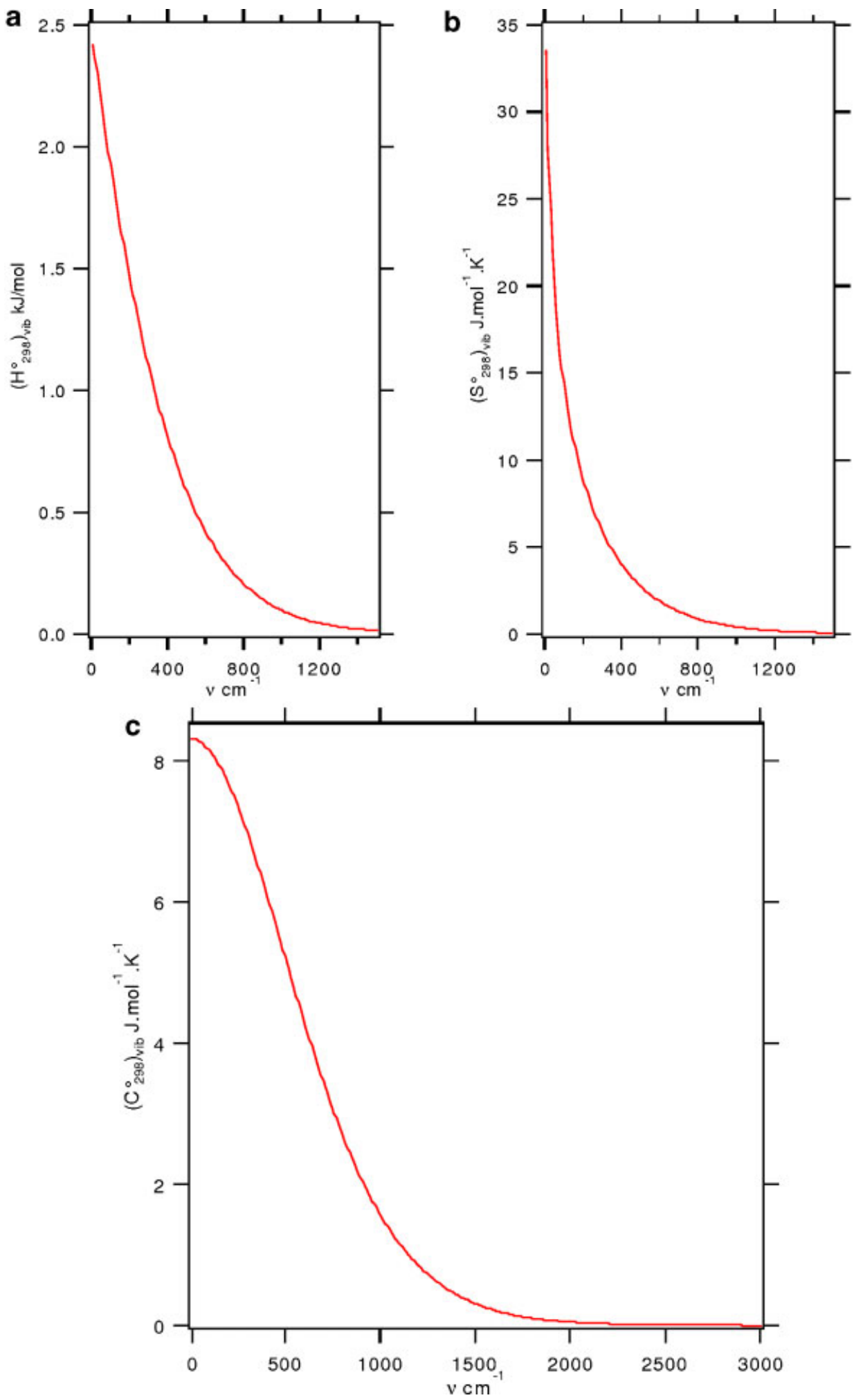

FIGURE 1. Plot of the contributions of a harmonic oscillator to enthalpy (a), entropy (b), and heat capacity (c), as a function of its wave number $v$. [Color figure can be viewed in the online issue, which is available at www.interscience.wiley.com.] 
2002; Chen \& Bozzelli, 2003; Hnizdo et al., 2003; Vansteenkiste et al., 2003, 2006; Sebbar, Bockhorn, \& Bozzelli, 2005; Da Silva, Kim, \& Bozzelli, 2006). However, improvements are still needed in the treatment of systems of coupled internal rotors (Van Speybroeck et al., 2005).

$f$. Role of the $V_{O}$ barrier. Due to the importance of hindered rotations in polyfunctional molecules, some comments concerning the contributions to thermochemical functions due to hindered rotation may be briefly presented. As shown in Figure 1, the vibrational contribution to enthalpy, entropy and heat capacity are more sensitive to low frequencies. It is thus expected that contributions due to hindered internal rotations should be dependent on the barrier height $V_{0}$. To illustrate this effect we report in Figure 2 the results of a statistical thermodynamic calculation on a fictitious hindered rotation of reduced moment $5 \times 10^{-39} \mathrm{~g} \mathrm{~cm}^{2}$ (i.e., $\sim 30 \mathrm{u}^{2}$ ) and of rotational barrier $V_{0}$ up to $100 \mathrm{~kJ} \mathrm{~mol}^{-1}$, at $T=298 \mathrm{~K}$. Exact calculations are indicated by red bold points and are compared with results obtained using the harmonic oscillator model, with the wave number given by Equation (35) (continuous blue lines), and using the free rotor approximation (green squares).

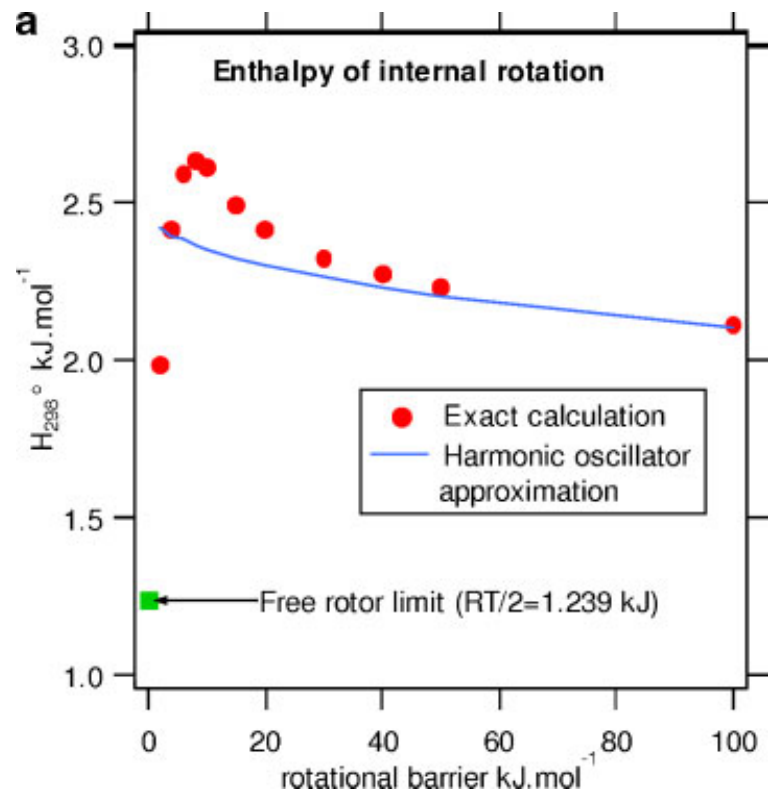

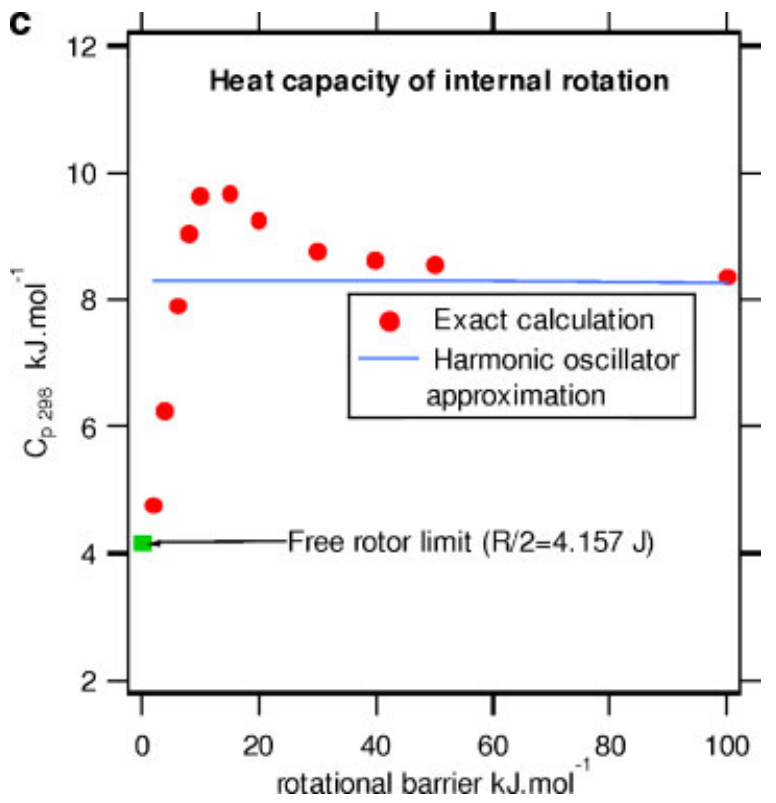

FIGURE 2. Contributions to (a) enthalpy, (b) entropy, and (c) heat capacity of one hindered rotation as a function of the rotational barrier $V_{0}$. [Color figure can be viewed in the online issue, which is available at www.interscience.wiley.com.] 
It is evident that hindered rotation has a more complex effect on the enthalpy and heat capacity than a simple harmonic vibration. It appears on Figure $2 \mathrm{a}$ and $\mathrm{c}$ that $H_{298}^{0}$ and $C_{p 298}$ vary from a minimum value for a free rotation $\left(V_{0}=0\right)$, then reach a maximum (for $V_{0} \sim 10 \mathrm{~kJ} \mathrm{~mol}^{-1}$ ), and decrease toward values given by the harmonic oscillator model at high $V_{0}$ values. The influence of the rotational barrier $V_{0}$ on the enthalpy contribution is limited to less than $0.5 \mathrm{~kJ} \mathrm{~mol}^{-1}$ when $V_{0}$ varies from 4 to $100 \mathrm{~kJ} \mathrm{~mol}^{-1}$ (Fig. 2a), and may be neglected to a first approximation. Such neglect is not advisable when considering entropy. As shown in Figure 2b, a change of 15 entropy unit is observed when the rotational barrier $V_{0}$ value passes from zero to $100 \mathrm{~kJ} \mathrm{~mol}^{-1}$. It appears that, for all $V_{0}$ values, the inequality $S_{298 \text { free.rot }}^{0}>S_{298 \text { hind.rot }}^{0}>S_{298 \text { harm.rot }}^{0}$ holds. Moreover, the limits of applicability of the various approximations may be easily delineated. Indeed Figure $2 b$ shows that, at room temperature, a rotation may be considered as free if $V_{0}$ is less than $\sim 4 \mathrm{~kJ} \mathrm{~mol}^{-1}$ and the harmonic oscillator approximation leads to $S_{298 \text { harm.rot }}^{0}$ which differ by less than $1 \mathrm{~J} \mathrm{~mol}^{-1} \mathrm{~K}^{-1}$ from $S_{298 \text { hind.rot }}^{0}$ if $V_{0}$ is larger than $\sim 20 \mathrm{~kJ} \mathrm{~mol}^{-1}$.

Another view of the influence of the barrier height $V_{0}$ on the entropy of a hindered rotor is presented in Figure 3. The plot is an example taken from the Pitzer and Gwinn tabulation where the two variables are the dimensionless ratio $V_{0} / R T$ and $1 / q_{\text {free }}$, with $q_{\text {free }}$ being the reciprocal of the partition function for free rotation, that is, $q_{\text {free }}=0.36\left(T I_{\text {red }}\right)^{1 / 2} / n$, if $I_{\text {red }}$ is expressed in u $\AA^{2}$ (Pitzer \& Gwinn, 1942). The shaded area presented in Figure 3 is limited by the curves corresponding to the fictive situations where (i) $I_{\text {red }}$ tends to infinity $\left(1 / q_{\text {free }}=0\right)$ and (ii) a light top ( $I_{\text {red }} \sim 1 \mathrm{u} \AA^{2}$ ) of symmetry number $n=3$ is attached to an infinite mass $\left(1 / q_{\text {free }}=0.5\right)$. This illustrates extend of the deviation introduced by the change in reduced moment of inertia on the entropy contribution.

The consequences of the change in rotational barrier height on the protonation entropy will be discussed in Calculation of Protonation Entropy.

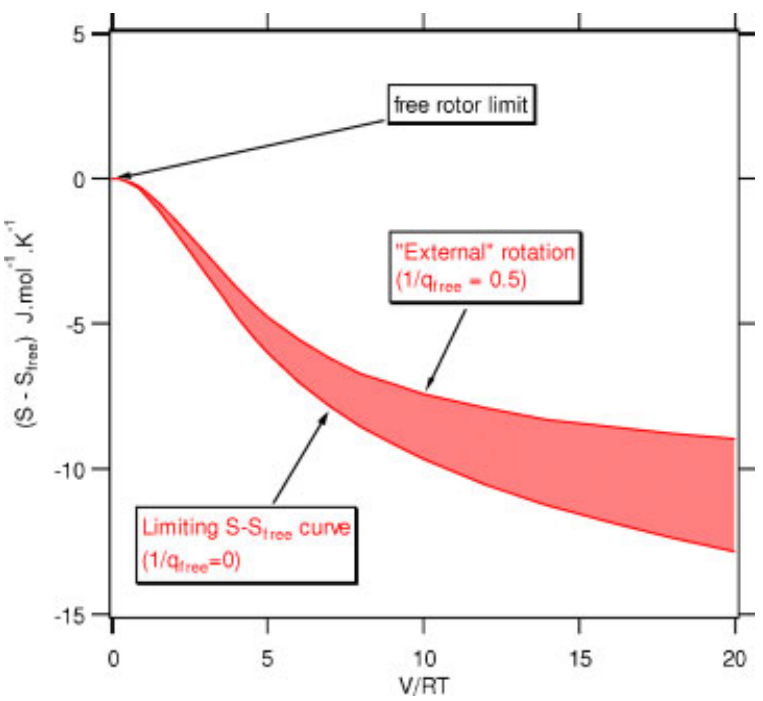

FIGURE 3. Pitzer results, illustrating the role of $1 / q$ on the entropy of a hindered rotation. [Color figure can be viewed in the online issue, which is available at www.interscience.wiley.com.] g. Additional remarks concerning entropy. The different contributions to absolute standard molar entropy and heat capacities are reflecting the molecular energy level densities. The translational energy levels are so dense that the translational energy may be considered as a continuum comprising infinitesimally close quantized levels. Rotational modes are also much close together than vibrational levels. As a consequence, the various contribution to entropies and heat capacities are always in the order: $S_{\text {trans }}^{0}>S_{\text {rot }}^{0}>S_{\text {vib }}^{0}>S_{\text {elec }}^{0}(\sim 0)$ and $C_{\text {trans }}^{0}>C_{\text {rot }}^{0}>$ $C_{\mathrm{vib}}^{0}>C_{\mathrm{elec}}^{0}(\sim 0)$. Some illustrative results are presented in Table 1.

Absolute entropy of $\mathrm{MH}^{+}$ions may be estimated by comparison with the experimental $S^{0}$ value of an isoelectronic neutral. For example $S^{0}\left(\mathrm{NH}_{4}{ }^{+}\right)$may be considered to be identical to $S^{0}\left(\mathrm{CH}_{4}\right), S^{0}\left(\mathrm{H}_{3} \mathrm{O}^{+}\right)$to $S^{0}\left(\mathrm{NH}_{3}\right)$ and so on. This approximation seems to work within $1-3 \mathrm{~J} \mathrm{~mol}^{-1} \mathrm{~K}^{-1}$ for small species without internal rotations, and after including the possible symmetry correction (East, Smith, \& Radom, 1997; Gal, Maria, \& Raczynska, 2001). For systems with internal rotations the approximation may lead to significant errors. This is particularly the case when the protonation on a heteroatom induces a bond lengthening which obviously lower the rotational barrier and increases the entropy of the corresponding species. For example the approximations $S^{0}\left[\left(\mathrm{CH}_{3}\right)_{2} \mathrm{OH}^{+}\right] \sim S^{0}\left[\left(\mathrm{CH}_{3}\right)_{2} \mathrm{NH}\right]$ (East, Smith, \& Radom, 1997) and $S^{0}\left(\mathrm{C}_{2} \mathrm{H}_{5} \mathrm{FH}^{+}\right) \sim S^{0}\left(\mathrm{C}_{2} \mathrm{H}_{5} \mathrm{OH}\right)$ (Bouchoux et al., 2001a) leads to underestimates in the entropy of the charged species of 10 and $20 \mathrm{~J} \mathrm{~mol}^{-1} \mathrm{~K}^{-1}$, respectively.

\section{Thermochemical Properties of Mixtures of Isomers}

For a mixture of $N$ isomers, the molar fractions $x_{i}$ of each component may be estimated assuming a Boltzmann distribution:

$$
x_{i}=\frac{\exp \left(-G_{i} / R T\right)}{\sum_{i}^{n} \exp \left(-G_{i} / R T\right)}
$$

which obviously needs the knowledge of the individual Gibbs free energies $G_{i}$.

Total value of a thermochemical quantity $X$ of $1 \mathrm{~mol}$ of mixture of $N$ components with molar fractions $x_{i}$ may be determined from the thermochemical properties of the separate species according to:

$$
\left(X_{T}^{0}\right)_{\text {total }}=\sum_{i}^{N} x_{i}\left(\mathrm{X}_{T}^{0}\right)_{I}
$$

The only exception is entropy because, if the components of the mixture are physically distinguishable, their permutation would lead to distinguishable configuration which would increase the entropy. This is at the origin of the entropy of mixing which should be added in the calculation of the total molar entropy of such a mixture:

$$
\left(S_{T}^{0}\right)_{\mathrm{total}}=\sum_{1}^{N} x_{i}\left(S_{T}^{0}\right)_{i}-R \sum_{1}^{N} x_{i} \ln x_{i}
$$

Consideration of the entropy of mixing is particularly important when the components of the mixture consist in 
TABLE 1. Contributions of translation, rotation and vibration to entropy $\left(\mathrm{J} \mathrm{mol}^{-1} \mathrm{~K}^{-1}\right)$ for representative molecules

\begin{tabular}{cccc}
\hline $\mathbf{M}$ & $\mathbf{S}_{\text {trans }}^{\circ}$ & $\mathbf{S}_{\text {rot }}^{\circ}$ & $\mathbf{S}_{\text {vib }}^{\circ}$ \\
\hline $\mathrm{Ar}$ & 154.7 & - & - \\
$\mathrm{N}_{2}$ & 150.4 & 41.2 & $\sim 0$ \\
$\mathrm{CS}_{2}$ & 162.1 & 65.9 & 9.7 \\
$\mathrm{C}_{2} \mathrm{H}_{4}$ & 150.4 & 66.5 & 2.6 \\
$\mathrm{CH}_{3} \mathrm{OH}$ & 152.1 & 79.6 & 7.9 \\
$\mathrm{C}_{2} \mathrm{H}_{5} \mathrm{OH}$ & 156.5 & 93.3 & 21.0 \\
$\mathrm{Catechol}$ & 167.4 & 116.6 & 63.9 \\
\hline
\end{tabular}

conformers, a common situation with floppy organic molecules. The magnitude of the entropy of mixing is not very sensitive to the actual proportions of the components (Guthrie, 2001) and the following approximations are generally done. It is readily verified that for $N$ equally populated conformations, $x_{i}=1 / N$ and thus the entropy of mixing reduces to $-R \ln (1 / N)$. It is also generally assumed that the entropy $S_{i}^{0}$ is the same for all conformations. Thus, calling $S_{\text {low }}^{0}$ the entropy of the conformer of lowest energy, the molar entropy may be approximated by

$$
S^{0}(\text { total }) \sim S_{\text {low }}^{0}+R \ln N
$$

Getting the correct number of distinguishable conformations, $N$, remains the only delicate task. First, it should be remembered that rotation around bonds to terminal symmetrical groups (e.g., $\mathrm{CH}_{3}, \mathrm{C}\left(\mathrm{CH}_{3}\right)_{3}$...) does not contribute to the entropy of mixing since the rotation leads to indistinguishable conformers. It is commonly assumed that rotation around a $\mathrm{C}(\mathrm{sp} 3)$ $\mathrm{C}(\mathrm{sp} 3)$ bond would lead to three different conformers. Despite the fact that it is still not clear that such a simplest enumeration law is always valid, even for alkanes (Tasi et al., 1998; Vansteenkiste et al., 2003), functional groups interactions may seriously perturb this expectation for polyfunctional molecules.

Finally, consideration of the entropy of mixing will obviously modify the Gibbs free energy of the mixture since combining $\Delta \mathrm{G}_{\mathrm{T}}{ }^{0}=\Delta \mathrm{H}_{\mathrm{T}}{ }^{0}-\mathrm{T} \Delta \mathrm{S}_{\mathrm{T}}{ }^{0}$ and Equation (43), it follows:

$$
\left(G_{T}^{0}\right)_{\text {total }}=\sum_{1}^{N} x_{i}\left(G_{T}^{0}\right)_{i}-R T \sum_{1}^{N} x_{i} \ln x_{i}
$$

\section{B. Experimental Methods of Determination of Gas-Phase Basicities}

\section{The Proton Transfer Reaction}

A thorough review of the various experimental techniques used in gas-phase ion thermochemistry appears five years ago (Ervin, 2001). Only the three methods relevant to the determination of gas-phase basicity or proton affinity (the "equilibrium" method, the "kinetic" method and the "thermokinetic" method) will be recalled here. A common characteristic of these three experimental procedures is that they are related to the thermochemistry of proton transfer reactions (i) between the molecule of interest, $\mathrm{M}$, and a reference bases, $\mathrm{B}_{i}$ :

$$
\mathrm{MH}^{+}+\mathrm{B}_{i} \rightarrow \mathrm{M}+\mathrm{BH}^{+}
$$

Thus, before describing each of these methods, the thermochemical relationship associated with reaction (i) will be first presented.

By definition, the standard Gibbs free energy of reaction (i) at temperature $T$ is given by

$$
\Delta_{i} G_{T}^{0}=G_{T}^{0}(\mathrm{M})+G_{T}^{0}\left(\mathrm{BH}^{+}\right)-G_{T}^{0}\left(\mathrm{MH}^{+}\right)-\mathrm{G}_{T}^{0}(\mathrm{~B})
$$

an equation which may be expressed in different ways. A first step is to introduce the enthalpy and entropy of reaction (i), $\Delta_{i} G_{T}^{0}=\Delta_{i} H_{T}^{0}-T \Delta_{i} S_{T}^{0}$, and then to develop each terms by reference to the temperature of $298 \mathrm{~K}$ :

$$
\Delta_{i} G_{T}^{0}=\Delta_{i} H_{298}^{0}+\int_{298}^{T} \Delta_{i} C_{p} \mathrm{~d} T-T\left[\Delta_{i} S_{298}^{0}+\int_{298}^{T} \frac{\Delta_{i} C_{p} \mathrm{~d} T}{T}\right]
$$

or, by introducing simplified notations for the integrals:

$$
\Delta_{i} G_{T}^{0}=\Delta_{i} H_{298}^{0}+\Delta_{i} H_{298 \rightarrow T}^{0}-T\left[\Delta_{i} S_{298}^{0}+\Delta_{i} S_{298 \rightarrow T}^{0}\right]
$$

We can now express $\Delta_{i} G_{T}^{0}$ as a function of the $298 \mathrm{~K}$ proton affinities of $\mathrm{M}$ and $\mathrm{B}$ :

$$
\begin{aligned}
\Delta_{i} G_{T}^{0}= & \mathrm{PA}_{298}(\mathrm{M})-\mathrm{PA}_{298}(\mathrm{~B})+\Delta_{i} H_{298 \rightarrow T}^{0} \\
& -T\left[\Delta_{i} S_{298}^{0}+\Delta_{i} S_{298 \rightarrow T}^{0}\right]
\end{aligned}
$$

or as a function of the $298 \mathrm{~K}$ gas-phase basicities:

$$
\begin{aligned}
\Delta_{i} G_{T}^{0}= & \mathrm{GB}_{298}(\mathrm{M})-\mathrm{GB}_{298}(\mathrm{~B})-(T-298) \Delta_{i} S_{298}^{0} \\
& +\Delta_{i} H_{298 \rightarrow T}^{0}-T \Delta_{i} S_{298 \rightarrow T}^{0}
\end{aligned}
$$

since

$\mathrm{GB}_{298}(\mathrm{M})-\mathrm{GB}_{298}(\mathrm{~B})=\mathrm{PA}_{298}(\mathrm{M})-\mathrm{PA}_{298}(\mathrm{~B})-298 \Delta_{i} S_{298}^{0}$ 
It must be recalled that, in the preceding expressions (4750), $\Delta_{i} S_{298}^{0}$ is the $298 \mathrm{~K}$ entropy change of reaction (i), that is:

$$
\begin{aligned}
\Delta_{i} S_{298}^{0}= & S_{298}^{0}(\mathrm{M})+S_{298}^{0}\left(\mathrm{BH}^{+}\right)-S_{298}^{0}\left(\mathrm{MH}^{+}\right) \\
& -S_{298}^{0}(\mathrm{~B})=\Delta_{p} S_{298}^{0}(\mathrm{~B})-\Delta_{p} S_{298}^{0}(\mathrm{M})
\end{aligned}
$$

As defined in Equation (48), the terms $\Delta_{i} H_{298 \rightarrow T}^{0}$ and $\Delta_{i} S_{298 \rightarrow T}^{0}$ are the thermal correction for enthalpy and entropy of reaction (i), respectively. Evaluation of these quantities involves integration of the difference in molar heat capacities at constant pressure $\Delta_{i} C_{p}=C_{p}(\mathrm{M})+C_{p}\left(\mathrm{~B}_{i} \mathrm{H}^{+}\right)-C_{p}\left(\mathrm{MH}^{+}\right)-C_{p}\left(\mathrm{~B}_{i}\right)$ which, because of the structural similarities of $\mathrm{MH}^{+}+\mathrm{B}_{i}$ in one hand and $\mathrm{M}+\mathrm{B}_{i} \mathrm{H}^{+}$in the other, is often assumed to essentially cancel to zero. In fact, this latter point is not always fulfilled when polydentate bases are considered, it is thus of some interest to illustrate this point. Figure 4 presents the evolution of the two $\Delta H_{298 \rightarrow T}^{0}$ and $\Delta S_{298 \rightarrow T}^{0}$ terms as a function of temperature in a $200-1,000 \mathrm{~K}$ range for a model reaction involving the locking of a free rotation. If this free rotor (of reduced moment of inertia equal to $30 \mathrm{u}^{2}$ ) becomes a rotation hindered by a barrier of $100 \mathrm{~kJ} \mathrm{~mol}^{-1}$, the $298 \mathrm{~K}$ variations of heat capacity, enthalpy and entropy are $\Delta_{i} C_{p} 298=4.2 \mathrm{~J} \mathrm{~mol}^{-1} \mathrm{~K}^{-1}, \Delta_{\mathrm{l}} H_{298}^{0}=0.87 \mathrm{~kJ} \mathrm{~mol}^{-1}$ and $\Delta_{i} S_{298}^{0}=-15.8 \mathrm{~J} \mathrm{~mol}^{-1} \mathrm{~K}^{-1}$, respectively. In the considered
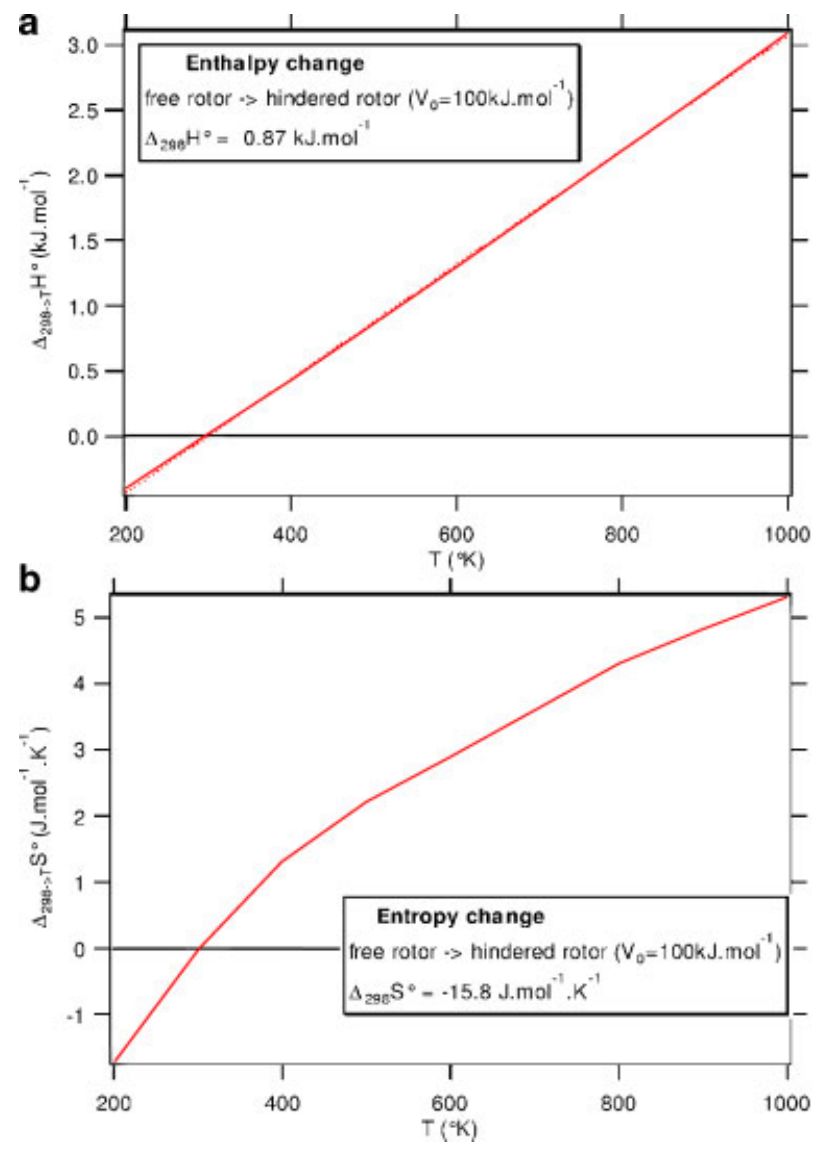

FIGURE 4. Thermal correction terms $\Delta H_{298 \rightarrow T}^{0}$ and $\Delta S_{298 \rightarrow T}^{0}$ calculated for a reaction involving the hindrance of a free rotation (potential energy barrier for the hindered rotor $V_{0}=100 \mathrm{~kJ} \mathrm{~mol}^{-1}$; reduced moment of inertia $=30 \mathrm{u}^{2}$ ). [Color figure can be viewed in the online issue, which is available at www.interscience.wiley.com.] temperature range, $\Delta_{i} C_{p}$ changes by less than $0.5 \mathrm{~J} \mathrm{~mol}^{-1} \mathrm{~K}^{-1}$, consequently $\Delta H_{298 \rightarrow T}^{0}$ is essentially linear as illustrated by Figure 4a while $\Delta S_{298 \rightarrow T}^{0}$ follows a logarithmic curve (Fig. 4b). It is noteworthy that, for this example, $\Delta_{i} H_{298 \rightarrow T}^{0}$ and $\Delta_{i} S_{298 \rightarrow T}^{0}$ are equal to $1.3 \mathrm{~kJ} \mathrm{~mol}^{-1}$ and $2.9 \mathrm{~J} \mathrm{~mol}^{-1} \mathrm{~K}^{-1}$ when $T=600 \mathrm{~K}$ These figures may be generally considered as negligible when compared to the experimental uncertainties, however, their existence may be kept in mind when several rotations are involved in a protonation process.

Assuming that $\Delta_{i} H_{298 \rightarrow T}^{0}$ and $\Delta_{i} S_{298 \rightarrow T}^{0}$ are effectively negligible, simplified expressions of the fundamental equations (49) and (50), may be written:

$$
\begin{gathered}
\Delta_{i} G_{T}^{0} \sim \mathrm{PA}_{298}(\mathrm{M})-\mathrm{PA}_{298}(\mathrm{~B})-T \Delta_{i} S_{298}^{0} \\
\Delta_{i} G_{T}^{0} \sim \mathrm{GB}_{298}(\mathrm{M})-\mathrm{GB}_{298}(\mathrm{~B})-(T-298) \Delta_{i} S_{298}^{0}
\end{gathered}
$$

These equations will be useful in the treatment of the data originating from experiment as it will be shown in the following sections.

\section{Equilibrium Measurements}

One of the most widely used quantitative method of determination of gas-phase basicities is the equilibrium method. This method is based on the determination of the equilibrium constant of the proton exchange reaction (i):

$$
\mathrm{MH}^{+}+\mathrm{B} \rightarrow \mathrm{M}+\mathrm{BH}^{+}
$$

At a given temperature $T$, the standard Gibbs free energy of reaction (i) is equal to:

$$
\Delta_{i} G_{T}^{0}=-R T \ln K_{i}
$$

where $K_{i}$ is the corresponding equilibrium constant:

$$
K_{i}=\frac{[\mathrm{M}]_{\mathrm{eq}}\left[\mathrm{BH}^{+}\right]_{\mathrm{eq}}}{\left[\mathrm{MH}^{+}\right]_{\mathrm{eq}}[\mathrm{B}]_{\mathrm{eq}}}
$$

When the four participants $\mathrm{M}, \mathrm{MH}^{+}, \mathrm{B}$, and $\mathrm{BH}^{+}$are allowed to react in conditions such that thermal equilibrium is attained, it is possible to determine directly the equilibrium constant $K_{i}$ by mass spectrometry techniques. The ratio of ion abundances at equilibrium $\left[\mathrm{BH}^{+}\right]_{\mathrm{eq}} /\left[\mathrm{MH}^{+}\right]_{\mathrm{eq}}$ is measured from peak intensities while the ratio of partial pressure (which are maintained constant) of the neutrals $p_{\mathrm{M}} / p_{\mathrm{B}}$ is separately determined. After determination of the actual temperature $T$ of the reactants, $\Delta_{i} G_{T}^{0}$ is directly obtained from Equation (54). The requirement of a sufficient number of collisions to thermalize the reactants may be accomplished by using high-pressure mass spectrometry (HPMS; McMahon \& Kebarle, 1985; Kebarle \& Chowdhury, 1987; Kebarle, 1988, 2003; McMahon, 1999; Mautner, 2003), ion cyclotron resonance (ICR; McIver, 1970; Bowers et al., 1971; Wolf et al., 1977; Aue \& Bowers, 1979; Larson \& McMahon, 1985; Bartmess, 1989; Kellersberger \& Dearden, 2003) or flow reactors (Bohme, MacKay, \& Schiff, 1980; Adams \& Smith, 1988; Bierbaum, 2003) experiments. Both pulsed HPMS and ICR experiments allows to verify that the equilibrium is reached by showing that $\left[\mathrm{BH}^{+}\right]_{\mathrm{eq}}$ and $\left[\mathrm{MH}^{+}\right]_{\mathrm{eq}}$ ion abundances became stationary at long reaction time. Examples of such behavior are presented in Figure 5 for a reaction clustering in an HPMS 
a
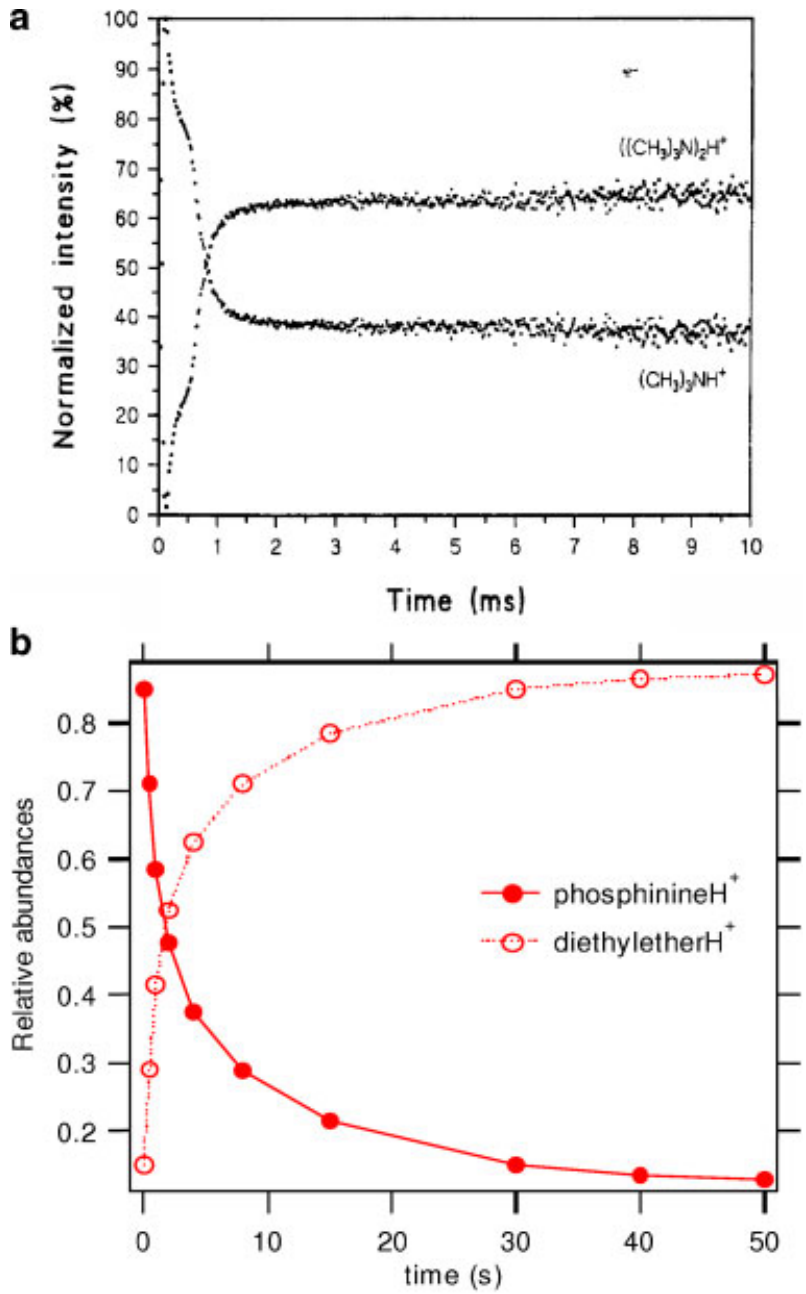

FIGURE 5. Examples of variation of ion intensities versus time showing the establishment of a chemical equilibrium (a) of protonated trimethylamine clustering [HPMS data from McMahon (1999), with permission from Springer, copyright 1999] and (b) proton transfer between phosphinine and diethylether. [Color figure can be viewed in the online issue, which is available at www. interscience.wiley.com.]

experiment and a proton exchange reaction (i) in an FT-ICR experiment.

The equilibrium method is obviously restricted to volatile and pure compounds, moreover the difference in basicity between $\mathrm{M}$ and $\mathrm{B}_{i}$ should be limited since too large (or too low) abundances ratio $\left[\mathrm{BH}^{+}\right]_{\mathrm{eq}} /\left[\mathrm{MH}^{+}\right]_{\mathrm{eq}}$ cannot be accurately determined experimentally due to limitation in the dynamic range of the instruments. Uncertainties on the $\Delta_{i} G_{T}^{0}$ derived from equilibrium measurement may originate from the uncertainties on (i) the measured signal intensities (particularly the smallest signal), (ii) the partial pressure of the neutrals, and (iii) the actual temperature $T$. Moreover, other sources of errors such as incomplete thermalization, isomerization, clustering, pyrolysis, non-equilibrium steady state, impurities and extraneous reactions may affect directly the measured $K$ value. These questions have been discussed by several authors (Mautner \& Sieck, 1991b; Harrison, 1997; Sieck, 1997; Gal, Maria, \& Raczynska, 2001; Mautner, 2003).
If the equilibrium constant for reaction (i) is measured at a fixed temperature $T$, the derived $\Delta_{i} G_{T}^{0}$ allows the determination of the gas-phase basicity and proton affinity of the unknown $\mathrm{M}$ only if an estimate of the entropy term $\Delta_{i} S^{0}$ is available. This is evidenced, for example, by the approximate equations (52) and (53): $\Delta_{i} G_{T}^{0} \sim \mathrm{PA}_{298}(\mathrm{M})-\mathrm{PA}_{298}(\mathrm{~B})-T \Delta_{i} S_{298}^{0} \sim \mathrm{GB}_{298}(\mathrm{M})-$ $\mathrm{GB}_{298}(\mathrm{~B})-(T-298) \Delta_{i} S_{298}^{0}$. As noted above, the protonation entropy of a polyfunctional molecule $\Delta_{p} S^{0}(\mathrm{M})$, and consequently $\Delta_{i} S^{0}$, may be significantly different from zero. In such situation, the measurement of the temperature dependency of the equilibrium constant is essential because it may lead to both the enthalpy and entropy change associated with reaction (i).

Accordingly, if a number of measurements of $K_{i}$ at variable temperature is available, a van't Hoff treatment of the data obtained by plotting $\ln K_{i}$ against $1 / T$ :

$$
\ln K_{i}=\frac{-\Delta_{i} G_{T}^{0}}{R T}=\frac{-\Delta_{i} H_{T}^{0}}{R T}+\frac{\Delta_{i} S_{T}^{0}}{R}
$$

can provide the enthalpic and entropic quantities $\Delta_{i} H_{T}^{0}$ and $\Delta_{i} S_{T}^{0}$ from the slope and the intercept of the fitted line. Two examples of this fitting procedure, applied to a proton transfer reaction, are presented in Figure 6 (Bouchoux et al., 2005).

Obviously, a van't Hoff treatment of reaction (i) assumes that the two thermochemical quantities $\Delta_{i} H_{T}^{0}$ and $\Delta_{i} S_{T}^{0}$ remain constant over the temperature range, a point not necessarily verified if $\mathrm{M}$ (or B) is a polyfunctional molecule since the heats capacities of reactants and products of the reaction not necessarily cancel (see above, Fig. 4).

A number of experimental thermochemical data has been obtained over a range of temperatures $\sim 250-650 \mathrm{~K}$ by the HPMS technique (McMahon, 1999; Mautner, 2003; Mautner, 2005). The accuracy obtained on the derived proton affinities and protonation entropies is still not clearly established. Accordingly, in addition to errors inherent to all equilibrium methods (i.e., the determination of partial pressure and true temperature $T$ ), systematic errors may be associated with van't Hoff data (Krug, Hunter, \& Grieger, 1976; Liu \& Guo, 2001) particularly when the entropy change is significant. If the corresponding deviation is

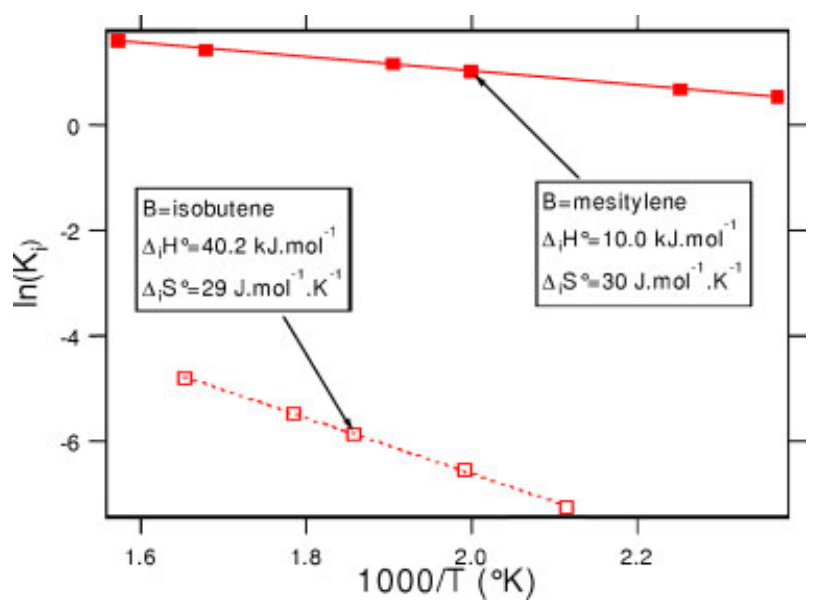

FIGURE 6. Examples of HPMS van't Hoff plots for proton transfer equilibria (i) $\mathrm{MH}^{+}+\mathrm{B} \rightarrow \mathrm{M}+\mathrm{BH}^{+}$involving $\mathrm{M}=3$-hydroxybutanone and two reference bases $\mathrm{B}=$ isobutene and mesitylene. [Color figure can be viewed in the online issue, which is available at www.interscience.wiley.com.] 
generally limited on the proton affinities, this may not be the case for the entropy since it is obtained from an extrapolated intercept, sensitive to a small deviation in the slope of the fitted line. If only the random error is considered, standard deviation may be as low as $1 \mathrm{~kJ} \mathrm{~mol}^{-1}$ for $\Delta_{i} H_{T}^{0}$ and $1 \mathrm{~J} \mathrm{~mol}^{-1} \mathrm{~K}^{-1}$ for $\Delta_{i} S_{T}^{0}$ as exemplified in Figure 6. However when entropy changes determined by different laboratories are compared, a poor agreement is generally observed, this is particularly true for polydentate bases where discrepancies as much as $50 \%$ may be observed (see Table 3 later on and the relevant discussion).

Beside the direct equilibrium method described above an alternative way to attain the equilibrium constant $K_{i}$ is to use the separate measurements of the forward and reverse rate constants for reaction (i) (Bohme, MacKay, \& Schiff, 1980):

$$
\begin{array}{llllll}
\mathrm{MH}^{+} & +\mathrm{B} & \rightarrow \mathrm{M} & +\mathrm{BH}^{+} & k_{\mathrm{f}} \\
\mathrm{M} & +\mathrm{BH}^{+} & \rightarrow \mathrm{MH}^{+} & +\mathrm{B} & k_{\mathrm{r}}
\end{array}
$$

Then, the principle of detailed balancing allows to write:

$$
K_{i}=\frac{k_{\mathrm{f}}}{k_{\mathrm{r}}}
$$

and to consequently estimate $\Delta_{i} G_{T}^{0}$ using Equation (54). This method gives correct results provided the reactants are at thermal equilibrium, and the partial pressure of the neutral is accurately determined, to estimate properly the bimolecular rate constants $k_{\mathrm{f}}$ and $k_{\mathrm{r}}$.

In flowing afterglow, FA, and selected ion flow tube, SIFT, techniques ionized and neutral reactants are entrained by a gas flow at a pressure of $\sim 1$ Torr and the reaction is followed by varying the velocity of the carrier gas, the rate constant is determined from the decay of the reactant ion. As an example, a precision better than $1 \mathrm{~kJ} \mathrm{~mol}^{-1}$ has been obtained on the gasphase basicity of $\mathrm{SiF}_{4}$ derived from SIFT experiments and use of Equations (54) and (57) (Ling et al., 1999). When using rate constants determined from FT-ICR experiments the forward and reverse rates method may also lead to a precision close to $1 \mathrm{~kJ} \mathrm{~mol}^{-1}$ on gas-phase basicities (Bouchoux \& Buisson, 2006).

\section{Kinetic Methods}

The "kinetic method," initiated 30 years ago by Cooks and coworkers (Cooks \& Kruger, 1977; McLuckey, Cameron, \& Cooks, 1981; Cooks et al., 1994; Cooks \& Wong, 1998; Cooks, Koskinen, \& Thomas, 1999; Zheng et al., 2003) is one of the most widely used mass spectrometry technique for the determination of thermochemical quantities in the gas phase. This success mainly lies on the operational simplicity of the method and its adaptability to most of the commercially available tandem mass spectrometers. Accordingly, the kinetic method has been initially developed in magnetic sector and triple quadrupole tandem instruments, it has been further used in quadrupole ion trap (Nourse \& Cooks, 1991) and ion cyclotron resonance (Decouzon et al., 1996) apparatus. By contrast the underlying theory of the method and the significance of the resulting data have been the subject of active discussions during the years. A brief recall is given below since the applicability of the kinetic method to the determination of the basicity of polyfunctional molecules has been hardly questioned.
To determine the protonation thermochemistry of a molecule $\mathrm{M}$, the kinetic method considers the competitive dissociations of a series of proton bound dimers $\left[\mathrm{MHB}_{i}\right]^{+}$, where $\mathrm{B}_{i}$ are reference bases:

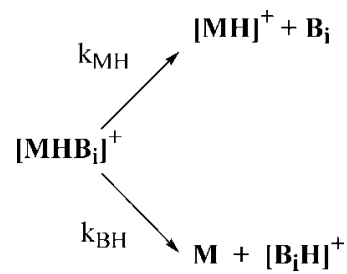

In practice, $\left[\mathrm{MHB}_{i}\right]^{+}$adduct ions are produced in a chemical ionization or an electrospray ion source and their dissociations (spontaneous or induced by collision) are analyzed after selection of the adduct ion by the first mass analyzer of the instrument or in the mass selection step of the sequence of events monitored in a trapping device.

The method generally supposes that the peak intensities $[\mathrm{MH}]^{+}$and $\left[\mathrm{B}_{\mathrm{i}} \mathrm{H}\right]^{+}$reflect the corresponding rate constants, that is, that we can write:

$$
\frac{[\mathrm{MH}]^{+}}{[\mathrm{BiH}]^{+}}=\frac{k_{\mathrm{MH}}}{k_{\mathrm{BH}}}
$$

Clearly, at this stage, a relationship between the rate constants $k_{\mathrm{MH}}$ and $k_{\mathrm{BH}}$ and the thermochemical quantities such as $\mathrm{GB}, \mathrm{PA}$ and $\Delta_{p} \mathrm{~S}^{0}$ is necessary. According to the absolute rate theory, the canonical rate constant $k$ associated with a population of species in thermal equilibrium at a given temperature $T$ is given by

$$
k=\left(\frac{k_{\mathrm{B}} T}{h}\right) \exp \left(\frac{-\Delta G^{\dagger}}{R T}\right)
$$

where $k_{\mathrm{B}}$ is the Boltzmann constant, $h$ the Planck constant, $R$ the gas constant and $\Delta G^{\dagger}$ is the free activation energy of the considered reaction. Then, according to Equation (60), the natural logarithm of the peaks ratio may be expressed by

$$
y_{i}=\ln \left(\frac{[\mathrm{MH}]^{+}}{\left[\mathrm{B}_{i} \mathrm{H}\right]^{+}}\right)=\ln \left(\frac{k_{\mathrm{MH}}}{k_{\mathrm{BH}}}\right)=\frac{\Delta G_{i}^{\ddagger}}{R T}
$$

with $\Delta G_{i}^{\ddagger}$ being the difference in Gibbs free energy between the transition structures of the two dissociation channels of Equation (58) (i.e., $\Delta G_{i}^{\dagger}=G^{0}$ (transition structure) BH $_{\mathrm{BH}}-G^{0}$ (transition structure $)_{\mathrm{MH}}$ ). If these transition structures are close to the corresponding final states, in structure and consequently in energy, Equation (61) may be simplified into:

$$
y_{i}=\ln \left(\frac{[\mathrm{MH}]^{+}}{\left[\mathrm{B}_{i} \mathrm{H}\right]^{+}}\right)=\frac{\Delta G_{i}}{R T}
$$

with

$$
\begin{aligned}
\Delta G_{i} & =G^{0}(\mathrm{M})+G^{0}\left(\mathrm{~B}_{i} \mathrm{H}^{+}\right)-G^{0}\left(\mathrm{MH}^{+}\right)-G^{0}\left(\mathrm{~B}_{i}\right) \\
& =\mathrm{GB}(\mathrm{M})-\mathrm{GB}\left(\mathrm{B}_{i}\right)
\end{aligned}
$$

at the temperature $T$. It is now essential to note that, since the system cannot be considered at thermal equilibrium, the 
temperature $T$ is an "effective temperature" $T_{\text {eff. }}$ Its meaning has been repeatedly commented (Vekey, 1996; Norrman \& McMahon, 1998; Drahos \& Vekey, 1999; Ervin, 2000, 2002). An approximation may be obtained using, for each of the fragmentations of Equation (58), the simplified microcanonical rate constant expression (the classical approximation of Rice, Rampsberger and Kassel):

$$
k(E)=v\left[\frac{E-E_{0}}{E}\right]^{(s-1)}
$$

where $v$ is the frequency factor, $E$ the internal energy of the dissociating species, $E_{0}$ the critical energy of the reaction and $s$ is the number of oscillators of the system (Ervin, 2000). If the internal energy $E$ of the adduct $\mathrm{MHB}_{i}^{+}$is much larger than the difference in critical energy between both processes, and if their frequency factors are identical, then the effective temperature of the system is given by

$$
T_{\text {eff }}=\frac{E-\left\langle E_{0}\right\rangle}{R(s-1)}
$$

with $\left\langle E_{0}\right\rangle$ being the mean critical energy of the two competitive channels (Eq. (58))

For our purpose, it is interesting to note that $T_{\text {eff }}$ (which will be noted simply $T$ here for the sake of brevity) is an empirical parameter directly related to the internal energy $E$ of the dissociating species $\mathrm{MHB}_{i}^{+}$. Its value can thus be changed by changing the activation conditions of the selected $\mathrm{MHB}_{i}^{+}$ ions.

Returning now to Equation (63), it clearly appears that the $\Delta G_{i}$ term is nothing else than the Gibbs free energy of reaction (i), $\Delta_{i} G_{T}^{0}$, which has been expressed by Equations (45)-(53). Consequently, by using Equation (49), $y_{i}$ may also be written:

$$
\begin{aligned}
y_{i}= & \frac{1}{R T}\left[\mathrm{PA}_{298}(\mathrm{M})-\mathrm{PA}_{298}\left(\mathrm{~B}_{i}\right)+\Delta_{i} H_{298 \rightarrow T}^{0}\right. \\
& \left.-T\left[\Delta_{i} S_{298}^{0}+\Delta_{i} S_{298 \rightarrow T}^{0}\right]\right]
\end{aligned}
$$

or, to a first approximation (from Eq. (52)):

$$
y_{i}=\frac{1}{R T}\left[\mathrm{PA}_{298}(\mathrm{M})-\mathrm{PA}_{298}\left(\mathrm{~B}_{i}\right)-T \Delta_{i} S_{298}^{0}\right]
$$

Under these circumstances, for a series of experiments using several bases $\mathrm{B}_{i}$ at temperature $T$, the correlation line $y_{i}=f\left(\mathrm{PA}_{298}\left(\mathrm{~B}_{i}\right)\right)$ would have a slope $1 / R T$ and an intercept with the $\mathrm{PA}_{298}$ scale (i.e., an apparent proton affinity $\mathrm{PA}_{\text {app }}$ ) equal to:

$$
\mathrm{PA}_{\text {app }}=\mathrm{PA}_{298}(\mathrm{M})+T\left\langle\Delta S_{i}^{0}\right\rangle
$$

where $\left\langle\Delta S_{i}^{0}\right\rangle$ is the mean value of the $\Delta S_{i}^{0}$ terms (which may be also written $\left.\Delta_{p} S^{0}(\mathrm{M})-\left\langle\Delta_{p} S^{0}\left(\mathrm{~B}_{i}\right)\right\rangle\right)$. A consequence of this later point is that a choice of a series of bases $\mathrm{B}_{i}$ of comparable $\Delta_{p} S^{0}\left(\mathrm{~B}_{i}\right)$ is desirable to reduce the mean deviation on the $\left\langle\Delta_{p} S^{0}\left(\mathrm{~B}_{i}\right)\right\rangle$ term. Alternatively, a variable change taking into account each individual $\Delta_{p} S^{0}\left(\mathrm{~B}_{i}\right)$ term:

$$
y_{i}^{\prime}=\ln \left(\frac{[\mathrm{MH}]^{+}}{\left[\mathrm{B}_{i} \mathrm{H}\right]^{+}}\right)-\Delta_{p} S^{0}\left(\mathrm{~B}_{i}\right)
$$

may obviate this requirement as suggested by Zheng and Cooks (2002). Under these circumstances, the $x$-axis intercept of the plot of $y^{\prime}$ versus $\mathrm{PA}\left(\mathrm{B}_{i}\right)$ becomes

$$
\mathrm{PA}_{\text {app }}^{\prime}=\mathrm{PA}_{298}(\mathrm{M})+T \Delta_{p} S^{0}(\mathrm{M})
$$

It has been noted however that the tabulated $\Delta_{p} S^{0}\left(\mathrm{~B}_{i}\right)$ values are often only estimated by comparison with similar compounds and are thus subjected to unexpected errors (Bouchoux et al., $2003 \mathrm{~b}$ ). It is sufficient to note that an uncertainty of $8 \mathrm{~J} \mathrm{~mol}^{-1} \mathrm{~K}^{-1}$ on $\Delta_{p} S^{0}\left(\mathrm{~B}_{i}\right)$ introduces an error of one unit in $y_{i}^{\prime}$, to consider carefully the use of this method.

In the original version of the kinetic method (also called the "standard" or "simple" kinetic method), it is assumed that $\mathrm{PA}_{298}(\mathrm{M})$ can be identified to the intercept $\mathrm{PA}_{\mathrm{app}}$. As Equation (68) shows, this approximation is valid only if $\left\langle\Delta S_{i}^{0}\right\rangle$ is negligible, that is, when $\left\langle\Delta_{p} S^{0}\left(\mathrm{~B}_{i}\right)\right\rangle$ and $\Delta_{p} S^{0}(\mathrm{M})$ fortunately cancel, a suggested mean to fulfill this condition is to consider for both $\mathrm{M}$ and $\mathrm{B}_{i}$, molecules pertaining to the same chemical class. Notwithstanding the fact that this latter concept is vague, such a procedure is practically limited to monofunctional bases $\mathrm{M}$ and $\mathrm{B}_{i}$ for which the individual $\Delta_{p} S^{0}$ terms are small. It cannot be applied to a polydentate base where the $\Delta_{p} S^{0}(\mathrm{M})$ term may be significant (of the order of several tens of $\mathrm{J} \mathrm{mol}^{-1} \mathrm{~K}^{-1}$ ) and of unexpected extent.

In general situations, it is necessary to treat both $\mathrm{PA}_{298}(\mathrm{M})$ and $\Delta_{p} S^{0}(\mathrm{M})$ as unknown and thus to use a more complete method employing the master equation (66), or its approximate form (67). The two parameters $\mathrm{PA}_{298}(\mathrm{M})$ and $\left\langle\Delta S^{0}\right\rangle$ can be derived from Equation (67) by using several sets of experiments corresponding to different effective temperatures $T_{j}$ (i.e., different ion activation conditions). Let us define $y_{i j}$ the $\ln \left([\mathrm{MH}]^{+} /\left[\mathrm{B}_{i} \mathrm{H}\right]^{+}\right)$values obtained from $n_{j}$ experiments differing in the adduct ion activation conditions and, for each $j$, the $n_{i}$ points corresponding to the number of reference bases $B_{i}$ :

$$
y_{i j}=\ln \left(\frac{[\mathrm{MH}]^{+}}{\left[\mathrm{B}_{i} \mathrm{H}\right]^{+}}\right)_{j}=\frac{\Delta S_{i}^{0}}{R}+\frac{\mathrm{PA}_{298}(\mathrm{M})-\mathrm{PA}_{298}\left(\mathrm{~B}_{i}\right)}{R T_{j}}
$$

This generalized form of Equation (67) constitutes the basis of the "extended" or "full entropy" kinetic method (Wu \& Fenselau, 1994; Cerda \& Wesdemiotis, 1996).

Data analysis in the extended kinetic method was initially done by considering two plots: first, the $y_{i}$ versus $\mathrm{PA}\left(\mathrm{B}_{i}\right)$ plots from which the slopes $1 / R T_{j}$ and the intercept $\left(\mathrm{PA}_{\mathrm{app}}\right)_{j}$ were deduced and, second, a pseudo-van't Hoff plot of $\left(\mathrm{PA}_{\mathrm{app}}\right)_{j} / R T_{j}$ versus $1 / R T_{j}$ which leads to $\mathrm{PA}_{298}(\mathrm{M})$ and $\left\langle\Delta S_{i}{ }^{0}\right\rangle / R$. A rigorous statistical procedure has been proposed to remove the correlation existing between the two variables of the second plot (Armentrout, 2000). Another, more straightforward, method for extracting thermochemical informations from the extended kinetic method consists to use directly the totality of the experimental observables $y_{i j}$ (Eq. (71)). Accordingly, the $y_{i j}$ versus $\mathrm{PA}_{298}\left(\mathrm{~B}_{i}\right)$ points may be fitted by a set of regression lines $\left(y_{i j}\right)_{\mathrm{calc}}=y_{0}+b_{j}\left(x_{0}-x_{i}\right)$ intersecting in a common point of coordinate $x_{0}=\mathrm{PA}_{\text {iso }}(\mathrm{M})$ and $y_{0}=\Delta S_{\text {iso }}^{0} / R$, called the "isothermal" point (Bouchoux et al., 2003a,b) or the "isoequilibrium" point (Ervin \& Armentrout, 2004) which is directly related to 
$\mathrm{PA}_{298}(\mathrm{M})$ and $\left\langle\Delta S_{i}{ }^{0}\right\rangle$ in the framework of the approximate equation (67):

$$
\begin{gathered}
x_{0}=\mathrm{PA}_{\text {iso }}(\mathrm{M})=\mathrm{PA}_{298}(\mathrm{M}) \\
y_{0}=\frac{\Delta S_{\text {iso }}^{0}}{R}=\frac{\left\langle\Delta S_{i}^{0}\right\rangle}{R}
\end{gathered}
$$

A statistical treatment of Equation (71), leading to $\mathrm{PA}_{\text {iso }}(\mathrm{M})$, $\Delta S_{\text {iso }}^{0} / R$ and the values of the $n_{j}$ effective temperatures $T_{j}$, has been proposed by Ervin and Armentrout (2004). The method is based on the orthogonal distance regression (ODR) method (Boggs et al., 1992), a least-square regression analysis which takes into account simultaneously all the $\left[n_{i}, n_{j}\right]$ data points.

Figure 7 illustrates the application of the kinetic method to $\mathrm{M}=$ butyronitrile, a simple monodentate base, for which a gasphase basicity of $767.8 \mathrm{~kJ} \mathrm{~mol}^{-1}$ has been obtained by the equilibrium method (Hunter \& Lias, 1998, 2005). Assuming a protonation entropy $\Delta_{p} S^{0}(\mathrm{M})$ of $6 \mathrm{~J} \mathrm{~mol}^{-1} \mathrm{~K}^{-1}$, Hunter and Lias $(1998,2005)$ proposed a proton affinity values PA(M) equal to $798.4 \mathrm{~kJ} \mathrm{~mol}^{-1}$. The data presented in Figure 7 were obtained from spontaneous (MIKE) and collision induced (CID-MIKE) dissociations of proton bound dimers occurring in the second field free of a B-E mass spectrometer (Cao \& Holmes, 1999). Reference bases $\mathrm{B}_{i}$ were also aliphatic cyanides for which the average entropic term $\left\langle\Delta_{p} S^{0}\left(\mathrm{~B}_{i}\right)\right\rangle$ amount to $5.2 \mathrm{~J} \mathrm{~mol}^{-1} \mathrm{~K}^{-1}$.

It appears consequently on this example that $\Delta_{p} S^{0}(\mathrm{M})$ and $\left\langle\Delta_{p} S^{0}\left(\mathrm{~B}_{i}\right)\right\rangle$ are practically identical. In such circumstances, $\left\langle\Delta S_{i}{ }^{0}\right\rangle$ is close to zero and the simple kinetic method, that is, the approximation $\mathrm{PA}_{298}(\mathrm{M})=\mathrm{PA}_{\mathrm{app}}$, is expected to be correct. Accordingly, as it is immediately visible in Figure 7, the MIKE and CID-MIKE experiments lead to essentially the same value. Moreover, the extended kinetic method, that is, the determination of the coordinates of the intercept of the two fitting lines, leads to a $\mathrm{PA}_{\text {iso }}$ value comparable to $\mathrm{PA}_{\mathrm{app}}$ and confirms that $\left\langle\Delta S_{i}{ }^{0}\right\rangle$ is negligible since $\Delta S_{\text {iso }}^{0} \sim 0$.

The situation is completely different when a bidentate base is considered. Figure 8 shows the kinetic method plots obtained

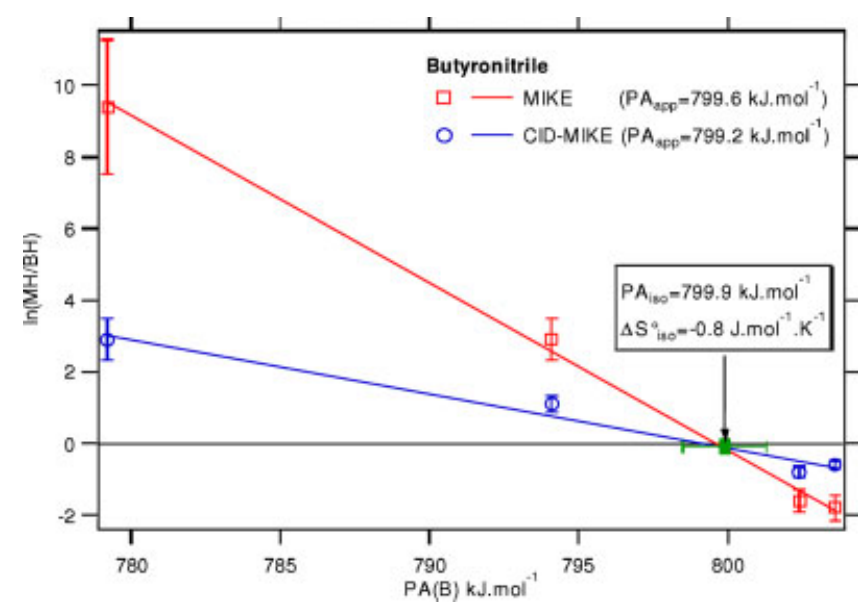

FIGURE 7. The "simple" $\left(\mathrm{PA}(\mathrm{M})=\mathrm{PA}_{\mathrm{app}}\right)$ and "extended" $\left(\mathrm{PA}(\mathrm{M})=\mathrm{PA}_{\text {iso }}\right)$ kinetic methods applied to a monodentate base $\mathrm{M}=$ butyronitrile. [Color figure can be viewed in the online issue, which is available at www.interscience.wiley.com.] for $\mathrm{M}=3$-aminopropanol. The protonation thermochemistry of this molecule has been examined by HPMS at variable temperature (Mautner et al., 1980). The authors reported a proton affinity, PA(3-aminopropanol) $=963.5 \mathrm{~kJ} \mathrm{~mol}^{-1}$, enhanced with respect to a monodentate homologue (e.g. PA(butylamine) $=$ $\left.921.5 \mathrm{~kJ} \mathrm{~mol}^{-1}\right)$, and a noticeable negative protonation entropy $\Delta_{p} S^{0}$ (3-aminopropanol) $=-43 \quad \mathrm{~J} \mathrm{~mol}^{-1} \mathrm{~K}^{-1}$, unambiguously showing a strong bifunctional interaction in the protonated form.

Experimental data used to construct Figure 8 were obtained with a triple quadrupole mass spectrometer equipped with an electrospray source and three series of experiments, corresponding to three different center of mass collision energies, $E_{\mathrm{cm}}$. It is evident from Figure 8, that the point of intercept of the three $y_{i j}=\ln \left(k_{\mathrm{MH}} / k_{\mathrm{BH}}\right)_{j}=\ln \left(\mathrm{MH} / \mathrm{B}_{i} \mathrm{H}\right)_{j}$ versus $\mathrm{PA}\left(\mathrm{B}_{i}\right)$ fitting lines is far from the $x$-axis and that the "simple" and "extended" kinetic methods cannot give the same results. Clearly, the apparent proton affinities given by the simple kinetic method, $\mathrm{PA}_{\mathrm{app}}$, is dependent on the amount of internal energy imparted to the adducts, that is, on the "effective" temperature $T$ as expected from Equation (68) when a significant $\left\langle\Delta S_{i}^{0}\right\rangle$ term exists. Thus the three $\mathrm{PA}_{\mathrm{app}}$ are wrong and obviously constitute underestimates of the true proton affinity. The isothermal point located using the ODR method, lead to $\mathrm{PA}_{\text {iso }}=954.0 \pm 3.4 \mathrm{~kJ} \mathrm{~mol}^{-1}$ in much closer agreement with the Mautner result. The protonation entropy derived from $\Delta S_{\text {iso }}^{0}$ is equal to $\Delta_{p} S^{0}$ (3-aminopropanol) $=-22 \pm 6 \mathrm{~J} \mathrm{~mol}^{-1} \mathrm{~K}^{-1}$ (for the set of reference bases considered $\left.\left\langle\Delta_{p} S^{0}\left(\mathrm{~B}_{i}\right)\right\rangle=-1.7 \mathrm{~J} \mathrm{~mol}^{-1} \mathrm{~K}^{-1}\right)$. As this example shown, the extended kinetic method is superior to the simple kinetic method in predicting the thermochemistry of polyfunctional molecules but it still gives PA and absolute values of $\Delta_{p} S^{0}$ lower than the equilibrium method. This point will be discussed below but one may immediately observe that the gas-phase basicities given by the equilibrium method (GB(3-aminopropanol $)=918.3 \mathrm{~kJ} \mathrm{~mol}^{-1}$ ) and the extended kinetic method $\left(\mathrm{GB}(3\right.$-aminopropanol $\left.)=915.0 \pm 3.8 \mathrm{~kJ} \mathrm{~mol}^{-1}\right)$ are fortunately in close agreement.

The difficulties of handling proton affinities and protonation entropies by the kinetic methods, particularly for polyfunctional molecules, have been the subject of active researches and discussions in the recent years. To test the validity of the hypothesis underlying the kinetic method and to delineate the limits of its applicability, two kinds of studies were envisaged, (i) microcanonical modelizations (Bojesen \& Breindahl, 1994; Nielsen \& Bojesen, 1995; Craig et al., 1997; Ervin, 2000, 2002; Laskin \& Futrell, 2000; Drahos \& Vekey, 2003; Bouchoux, Sablier, \& Berruyer-Penaud, 2004; Drahos, Pelt, \& Veckey, 2004; Grabowy \& Mayer, 2004) and (ii) experimental assessments (Wang et al., 1999; Cao, Aubry, \& Holmes, 2000; Hahn \& Wesdemiotis, 2003; Bouchoux et al., 2003a,b; Wesdemiotis, 2004; Bouchoux, 2006b)

Since an ensemble of isolated ions dissociating in a mass spectrometer cannot be characterized by a thermal distribution of energies but rather constitutes a microcanonical ensemble, it seems more appropriate to express the rate constants appearing in Equation (58) by:

$$
k\left(E, E_{0}, J\right)=\frac{\sum P^{\ddagger}\left(E-E_{0}, J\right)}{h N(E, J)}
$$




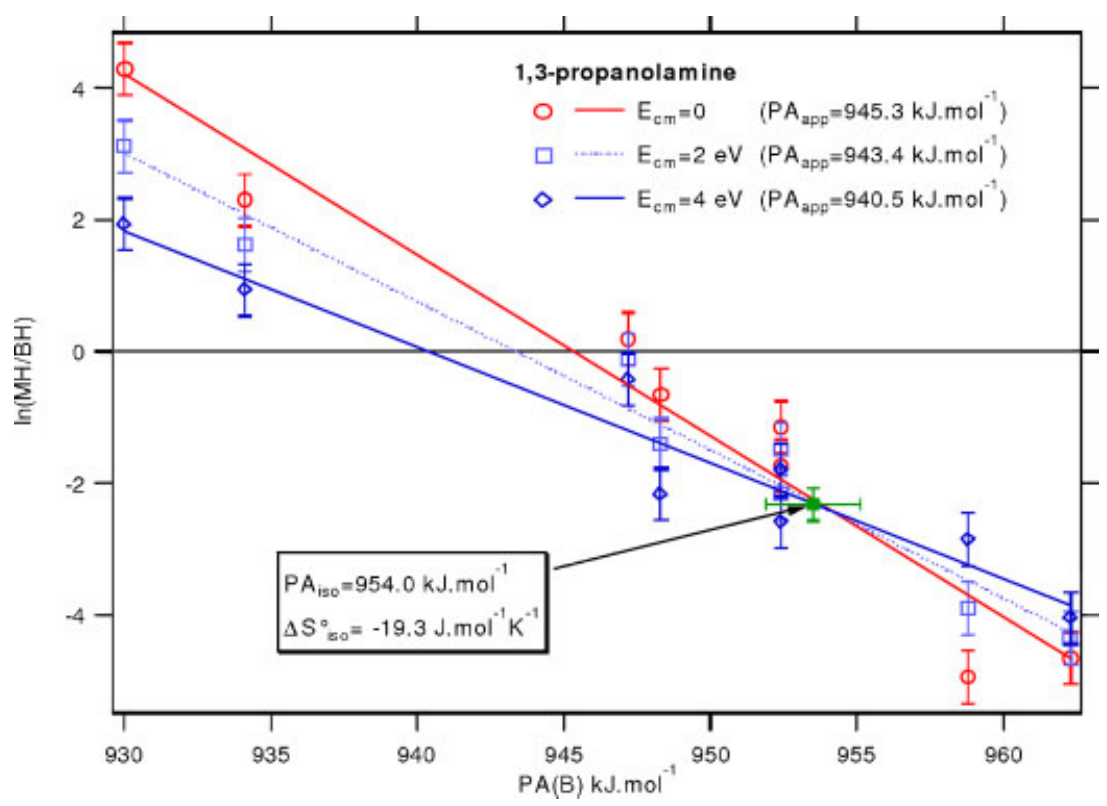

FIGURE 8. The "simple" $\left(\mathrm{PA}(\mathrm{M})=\mathrm{PA}_{\mathrm{app}}\right)$ and "extended" $\left(\mathrm{PA}(\mathrm{M})=\mathrm{PA}_{\text {iso }}\right)$ kinetic methods applied to a bidentate base $\mathrm{M}=1,3$-aminopropanol. [Color figure can be viewed in the online issue, which is available at www.interscience.wiley.com.]

where $\sum P^{\star}\left(E-E_{0}, J\right)$ is the sum of states of the transition structure with a total energy $E-E_{0}$ and with an angular momentum $J, N(E, J)$ is the density of states of the proton bound dimer at an energy $E$ and with angular momentum $J$ and $h$ is the Planck's constant (Steinfeld, Francisco, \& Hase, 1989; Gilbert \& Smith, 1990; Baer \& Hase, 1996). Calculation of the ratio of ion abundances $[\mathrm{MH}]^{+} /\left[\mathrm{B}_{i} \mathrm{H}\right]^{+}$rigorously needs the integration of kinetic expressions including the experimental time window over the internal energy and angular momentum distribution of the ions (Bojesen \& Breindahl, 1994; Nielsen \& Bojesen, 1995; Ervin, 2000, 2002; Drahos \& Vekey, 2003; Drahos, Pelt, \& Vekey, 2004). Other approaches used the approximation $[\mathrm{MH}]^{+} /$ $\left[\mathrm{B}_{i} \mathrm{H}\right]^{+}=k_{\mathrm{MH}} / k_{\mathrm{BH}}$ (Eq. (59)) and evaluated the rate constant at a single value corresponding to the most probable internal energy (Craig et al., 1997; Laskin \& Futrell, 2000; Bouchoux, Sablier, $\&$ Berruyer-Penaud, 2004). Extensive simulations have been made aiming to reproduce dissociation of metastable ions and species activated by single or multiple collisions of up to $100 \mathrm{eV}$ laboratory energies. All these various microcanonical approaches reveal that the kinetic method plots of $\ln \left([\mathrm{MH}]^{+} /\right.$ $\left[\mathrm{B}_{i} \mathrm{H}\right]^{+}$) versus $\Delta H^{0}$, where $\Delta H^{0}$ is the difference in enthalpy of the two sets of dissociation products, are nearly linear but can exhibit significant curvature. Figure 9 shows an example of kinetic plots obtained for a system characterized by a $\Delta S_{298}^{0}=-34 \mathrm{~J} \mathrm{~mol}^{-1} \mathrm{~K}^{-1}$ and three different excitation energies of the proton bond dimer ( $E^{*}$ denotes the internal energy $\mathrm{E}$ corresponding to a rate constant $k$ equal to $10^{5} \mathrm{~s}^{-1}$ ) (Bouchoux, Sablier, \& Berruyer-Penaud, 2004). The $\ln \left([\mathrm{MH}]^{+} /\left[\mathrm{B}_{i} \mathrm{H}\right]^{+}\right)$, which is approximated here by $\ln \left(k_{\mathrm{MH}} / k_{\mathrm{BH}}\right)$, demonstrates indeed curvatures as evidenced by a polynomial fitting. Note that the intercept of the three curves arises close to $\Delta H^{0}=0$ and for a $y$-value close to -4 , that is, for a $\Delta S_{\text {iso }}^{0} \sim-33 \mathrm{~J} \mathrm{~mol}^{-1} \mathrm{~K}^{-1}$ as expected from the model. This agreement is however not general and extensive simulations (Drahos \& Vekey, 2003; Drahos, Pelt,
\& Vekey, 2004; Ervin \& Armentrout, 2004) established that the proton affinities and protonation entropies determined by the extended kinetic method would present noticeable systematic errors particularly when $\Delta S^{0}$ is larger than $\sim 30 \mathrm{~J} \mathrm{~mol}^{-1} \mathrm{~K}^{-1}$.

Using model systems characterized by entropy differences $\Delta S^{0}$ situated between -36 to $+68 \mathrm{~J} \mathrm{~mol}^{-1} \mathrm{~K}^{-1}$ in a microcanonical simulation of the extended kinetic method plot, Ervin and Armentrout (2004) were able to demonstrate errors up to $12 \mathrm{~kJ} \mathrm{~mol}^{-1}$ for ion affinities and up to $30 \mathrm{~J} \mathrm{~mol}^{-1} \mathrm{~K}^{-1}$ for entropy by using the extended kinetic method. Moreover, the authors show that these systematic errors are larger for systems with large entropy differences as shown in Figure 10.

Similar findings were also reported by Drahos, Pelt, and Vekey (2004) in a simulation of the kinetic method based on 3,000 different model systems. Again, as illustrated by Figure 11, systematic errors on $\Delta H^{0}$ (i.e., $\left.\mathrm{PA}(\mathrm{M})_{\text {exact }}-\mathrm{PA}(\mathrm{M})_{\text {kinetic }}\right)$ and $\Delta S^{0}$ (i.e., $\left.\Delta S^{0}(\mathrm{M})_{\text {exact }}-\Delta S^{0}(\mathrm{M})_{\text {kinetic }}\right)$ are observed and shown to be dependent on the value of $\Delta S^{0}$.

In the same study Drahos et al. also shown that a strong correlation exists between $\Delta H_{\text {error }}^{0}$ and $\Delta S_{\text {error }}^{0}$ with the consequence that errors of less than $4 \mathrm{~kJ} \mathrm{~mol}^{-1}$ were predicted on the corresponding Gibbs free energy difference $\Delta G$ (Fig. 12).

These theoretical expectations were confirmed by considering experimental thermochemical data concerning a series of $\sim 30$ polyfunctional molecules for which negative protonation entropies in the range 0 to $-97 \mathrm{~J} \mathrm{~mol}^{-1} \mathrm{~K}^{-1}$ were reported (Bouchoux, 2006b). A comparison of the proton affinities and protonation entropies determined by the extended kinetic method using the isothermal point $\left(\mathrm{PA}_{\text {iso }}\right.$ and $\left.\Delta_{p} S_{\text {iso }}^{0}\right)$ and by the equilibrium method at variable temperature $\left(\mathrm{PA}_{\text {equ }}\right.$ and $\left.\Delta_{p} S_{\text {equ }}^{0}\right)$ shows systematic underestimates on $\mathrm{PA}_{\text {iso }}$ and on the absolute value of $\Delta_{p} S_{\text {iso }}^{0}$. Moreover, the differences $\left(\mathrm{PA}_{\text {iso }}-\mathrm{PA}_{\text {equ }}\right)$ and $\left(\Delta_{p} S_{\text {iso }}^{0}-\Delta_{p} S_{\text {equ }}^{0}\right)$ are clearly related to the extent of $\Delta_{p} S_{\text {equ }}^{0}$ as illustrated by Figure 13 . 


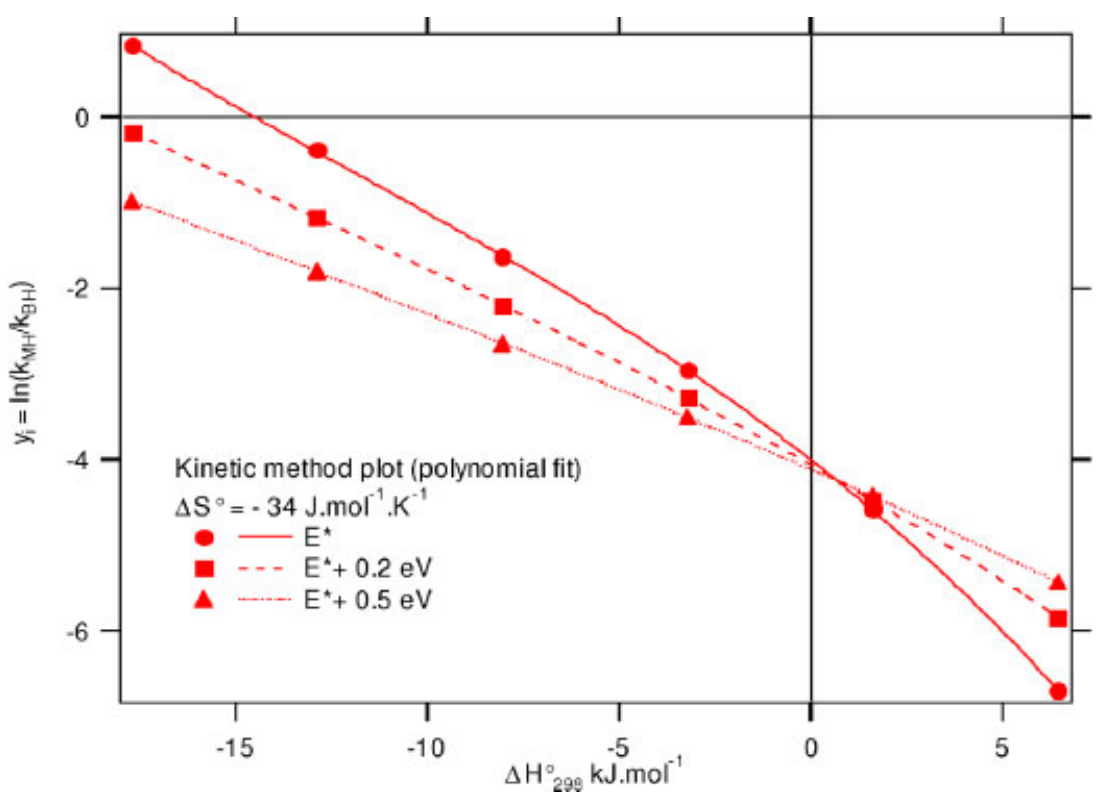

FIGURE 9. Kinetic method plots for a model system corresponding to a $\Delta S_{298}^{0}$ term equal to $-34 \mathrm{~J} \mathrm{~mol}^{-1} \mathrm{~K}^{-1}$. [Color figure can be viewed in the online issue, which is available at www.interscience. wiley.com.]

As shown above in Figure 12 for model systems, another mean to present these systematic deviations is to correlate the difference in enthalpy with the entropy difference. This is exemplified in Figure 14a for the set of real experiments (Bouchoux et al., 2006b). This approximately linear enthalpyentropy relationship is in fact an illustration of an enthalpyentropy compensation effect, a well-known propagation of error effect occurring in the analysis of van't Hoof and Arrhenius data (Krug, Hunter, \& Grieger, 1976; Exner, 1988; Liu \& Guo, 2001).

Fortunately, and as also predicted from Figure 12, because $\Delta G=\Delta H-T \Delta S$ it is expected that gas-phase basicities would exhibit lower discrepancies than the individual $\Delta H$ and $\Delta S$ terms. Indeed, when considering the same set of $\sim 30$ experiments, the gas-phase basicity given by the extended kinetic method, calculated from $\mathrm{GB}_{\text {iso }}=\mathrm{PA}_{\text {iso }}-298 \times 10^{-3}\left[108.8-\Delta_{p} S_{\text {iso }}^{0}\right]$ is close to the "true" $\mathrm{GB}_{\text {equ }}$ values obtained from equilibrium measurements at variable temperature (Fig. 14b). It is noteworthy that the overall mean deviation, as obtained from the difference $\left(\mathrm{GB}_{\text {iso }}-\mathrm{GB}_{\text {equ }}\right)$, represents only $2.4 \mathrm{~kJ} \mathrm{~mol}^{-1}$ (Bouchoux, 2006b).

In conclusion, the present status of our knowledge suggests that the kinetic method should be carefully used when polyfunctional bases are considered. First of all, the extended method should be imperatively used with a correct statistical treatment of a correct set of experiments. Nevertheless, underestimates of $\mathrm{PA}_{\text {iso }}(\mathrm{M})$ and $\Delta_{p} S_{\text {iso }}^{0}(\mathrm{M})$ may be obtained if the latter term is significantly negative. Only the $\mathrm{GB}_{\text {iso }}(\mathrm{M})$ obtained by the combination of both $\mathrm{PA}_{\mathrm{iso}}(\mathrm{M})$ and $\Delta_{p} S_{\text {iso }}^{0}(\mathrm{M})$ is expected to be accurate within a few $\mathrm{kJ} \mathrm{mol}^{-1}$.

\section{Thermokinetic Method}

A qualitative approach of gas-phase basicities is given by the "bracketing" technique (Bartmess, 2003). This method is based on the occurrence or no-occurrence of an ion-molecule reaction and the assumption that the reaction will be observed only if its Gibbs free energy change is $<0$. Thus, for a proton transfer reaction such as (if), it is concluded that $\mathrm{GB}(\mathrm{M})<\mathrm{GB}(\mathrm{B})$ if $\mathrm{BH}^{+}$ is formed when $\mathrm{MH}^{+}$is allowed to react with a base $\mathrm{B}$. Conversely, $\mathrm{GB}(\mathrm{M})$ is assumed to be higher than $\mathrm{GB}(\mathrm{B})$ if no reaction at all occurs:

$$
\begin{aligned}
& \mathrm{MH}^{+}+\mathrm{B} \rightarrow \mathrm{M}+\mathrm{BH}^{+} \\
& \mathrm{YES}=\mathrm{GB}(\mathrm{M})<\mathrm{GB}(\mathrm{B}) \\
& \mathrm{NO}=\mathrm{GB}(\mathrm{M})>\mathrm{GB}(\mathrm{B})
\end{aligned}
$$

This rule neglects the effects of the end- or ex-ergonic character of the reaction and the influence of the temperature on the reaction rate. Another limitation is the choice of the threshold of reaction efficiency chosen to decide whether a reaction is occurring or not. The bracketing technique should be consequently considered as an approximate method. It may be however useful when equilibrium or kinetic methods are not practicable, for example when $M$ is an unstable or nonvolatile compound. Tentative of quantification of the bracketing method have been proposed in the 1980-1990 (Bohme, MacKay, \& Schiff, 1980; Mautner, 1982; Gorman \& Amster, 1993; Wu \& Lebrilla, 1993; Zhang et al., 1993) and refined more recently in an operational formalism called the "thermokinetic" method (Bouchoux, Salpin, \& Leblanc, 1996; Bouchoux, 2006a).

The thermokinetic method is based on a correlation between the bimolecular rate of the above reaction (if) and the corresponding standard free energy change $\Delta_{i} G^{0}$. The basis of the method is to consider that a proton transfer from $\mathrm{MH}^{+}$to $\mathrm{B}_{i}$ (reaction if) occurs via a single reaction intermediate 

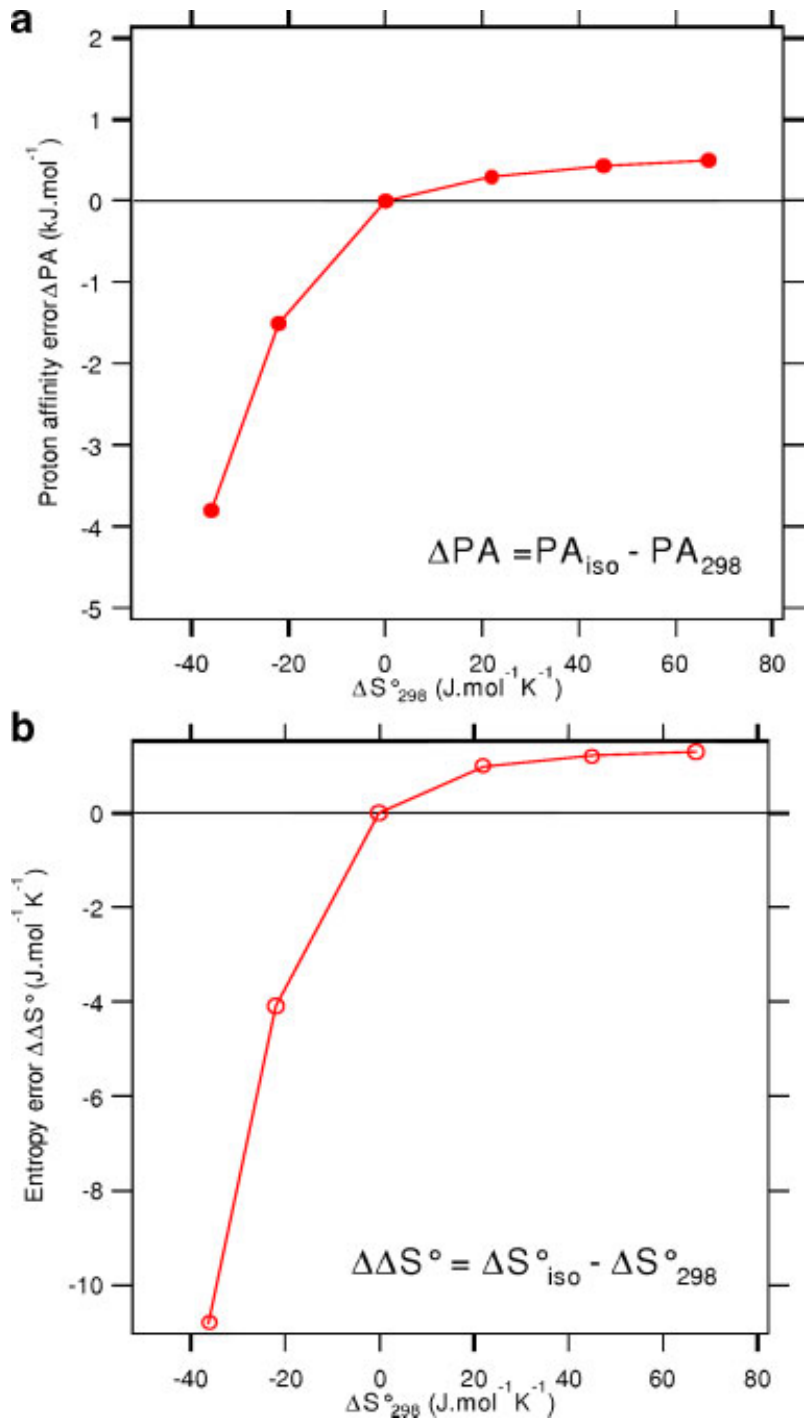

FIGURE 10. Calculated systematic errors in (a) proton affinity $(\triangle \mathrm{PA})$ and (b) protonation entropy $\left(\Delta \Delta S^{0}\right)$, determined by the isothermal point method as a function of the true protonation entropy difference $\Delta S_{298}^{0}$. Reproduced from Ervin 2004, with permission from John Wiley, Ltd., copyright 2004. [Color figure can be viewed in the online issue, which is available at www.interscience.wiley.com.]

$\left[\mathrm{MHB}_{i}\right]^{+}$:

$$
\mathrm{MH}^{+}+\mathrm{B}_{i} \stackrel{k_{\text {coll }}}{\longrightarrow}\left[\mathrm{MHB}_{i}\right]^{+} \stackrel{k_{1}}{\longrightarrow} \mathrm{M}+\mathrm{B}_{i} \mathrm{H}^{+}
$$

Applying the steady-state approximation to this intermediate, the reaction efficiency RE, that is, the ratio of the experimental rate constant, $k_{\text {exp }}$ determined from the $\mathrm{MH}^{+}$decay, to the collision rate constant, $k_{\mathrm{coll}}$, can be expressed by Equation (75):

$$
\mathrm{RE}=\frac{k_{\text {exp }}}{k_{\text {coll }}}=\left[1+\frac{k_{-1}}{k_{1}}\right]^{-1}
$$

where $k_{-1}$ and $k_{1}$ are unimolecular rate constants for the backward and forward dissociations of the intermediate ion $\left[\mathrm{MHB}_{i}\right]^{+}$.

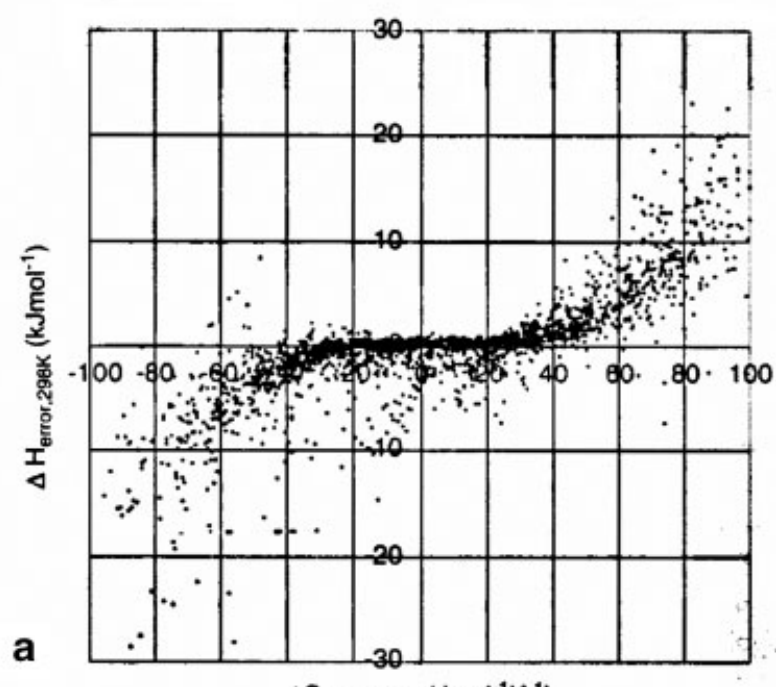

$\Delta \mathrm{S}_{\text {theor,298K }}\left(\mathrm{Jmol}^{-1} \mathrm{~K}^{-1}\right)$

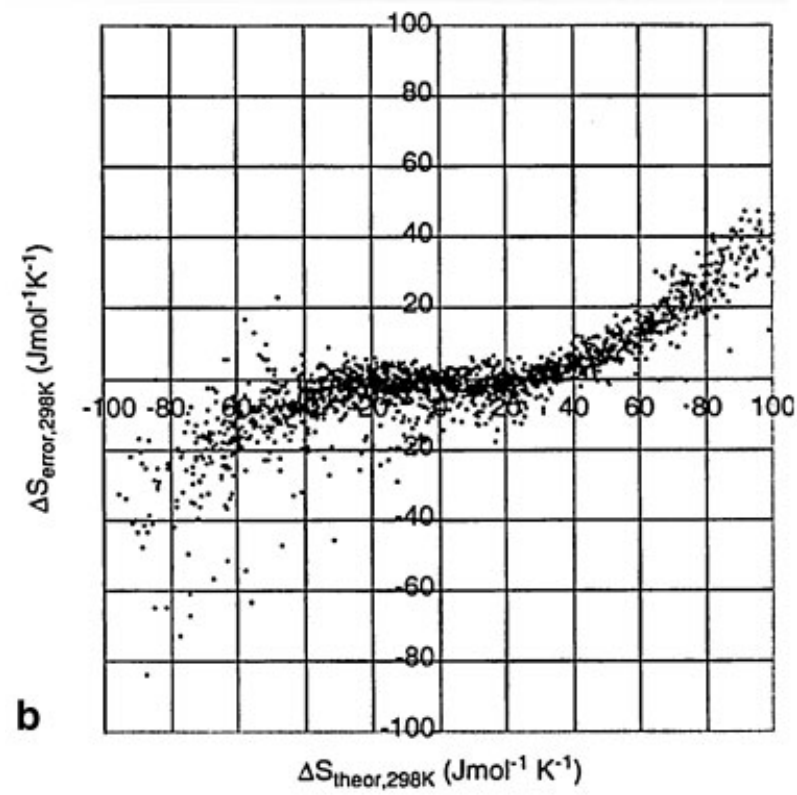

FIGURE 11. Random modeling of the errors on (a) enthalpy $\Delta H_{\mathrm{error}}^{0}=$ $\mathrm{PA}(\mathrm{M})_{\text {exact }}-\mathrm{PA}(\mathrm{M})_{\text {kinetic }}$ and (b) entropy $\Delta S_{\text {error }}^{0}=\Delta S^{0}(\mathrm{M})_{\text {exact }}-$ $\Delta S^{0}(\mathrm{M})_{\text {kinetic }}$ as a function of the exact entropy term called $\Delta S^{0}(\mathrm{M})_{\text {theor }}$ in the graphs. Reproduced from Drahos, Pelt, and Vekey (2004), with permission from John Wiley \& Sons Ltd., copyright 2004.

By using the canonical thermodynamic formulation of the transition state theory (Eq. (60)), the reaction efficiency becomes

$$
\mathrm{RE}=\left[1+\exp \left(\frac{\Delta_{i} G_{T}^{\ddagger}}{R T}\right)\right]^{-1}
$$

with $\Delta_{i} G_{T}^{\ddagger}$ being the difference in Gibbs free energy between the transition state of the two dissociation channels, that is, $\Delta_{i} G_{T}^{\ddagger}=$ $\left.\left.G_{0}^{\ddagger}\left(\mathrm{MHB}_{i}\right]^{+} \rightarrow \mathrm{M}+\mathrm{B}_{i} \mathrm{H}^{+}\right)-G_{0}^{\ddagger} \quad\left(\mathrm{MHB}_{i}\right]^{+} \rightarrow \mathrm{MH}^{+}+\mathrm{B}_{i}\right)$ at temperature $T$. If these transition states are close in energy and 


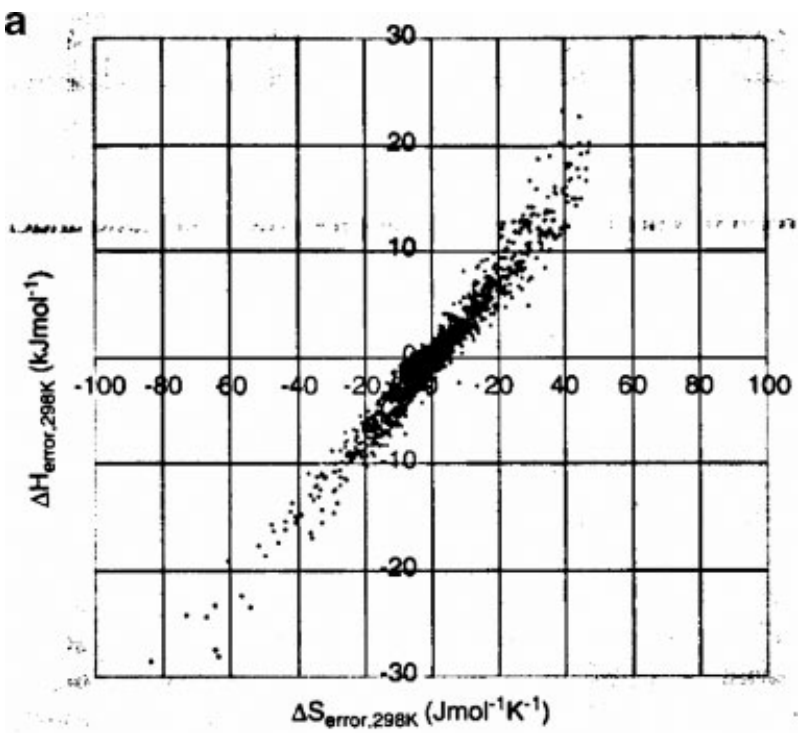

b

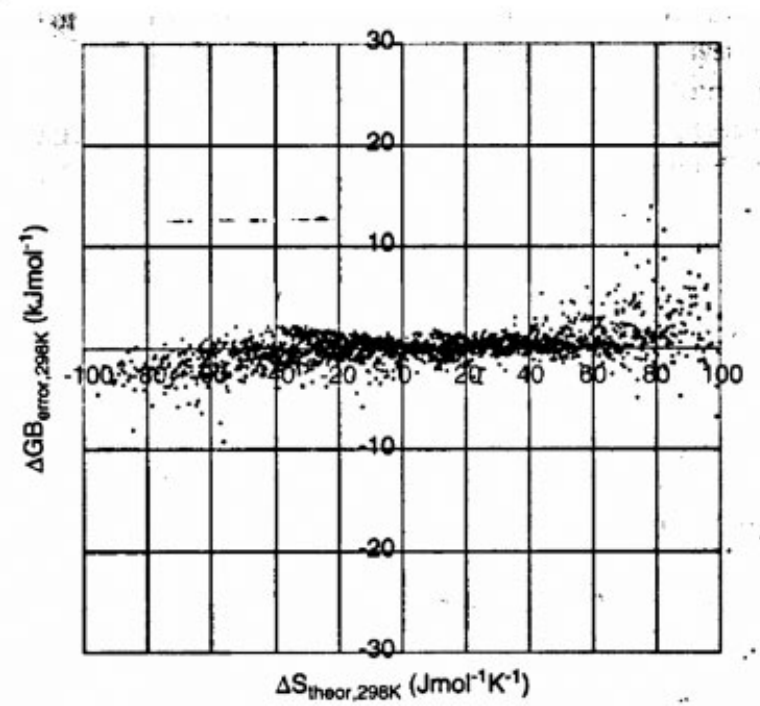

FIGURE 12. Cross correlation between $\Delta H_{\mathrm{error}}^{0}$ and $\Delta S_{\mathrm{error}}^{0}$ and random modeling of the errors on Gibbs free energy $\Delta \mathrm{GB}_{\text {error }}^{0}=\mathrm{GBA}(\mathrm{M})_{\text {exact }}-$ $\mathrm{GB}(\mathrm{M})_{\text {kinetic }}$ as a function of the exact entropy term called $\Delta S^{0}(\mathrm{M})_{\text {theor }}$ in the graphs. Reproduced from Drahos, Pelt, and Vekey (2004), with permission from John Wiley \& Sons Ltd., copyright 2004.

in structure to the corresponding final states, Equation (76) may be simplified to:

$$
\mathrm{RE}=\left[1+\exp \left(\frac{\Delta_{i} G_{T}^{0}}{R T}\right)\right]^{-1}
$$

with

$$
\Delta_{i} G_{T}^{0}=G_{T}^{0}(\mathrm{M})+G_{T}^{0}\left(\mathrm{~B}_{\mathrm{i}} \mathrm{H}^{+}\right)-G_{T}^{0}\left(\mathrm{MH}^{+}\right)-G_{T}^{0}\left(\mathrm{~B}_{\mathrm{i}}\right)
$$

which, again, corresponds to the Gibbs free energy change of reaction (i), that is, $\Delta_{i} G_{T}^{0}$ (see Eqs. 45-53). We can thus use Equations (49) and (50) (or their simplified versions 52 and 53) to establish a link between the reaction efficiency RE and the

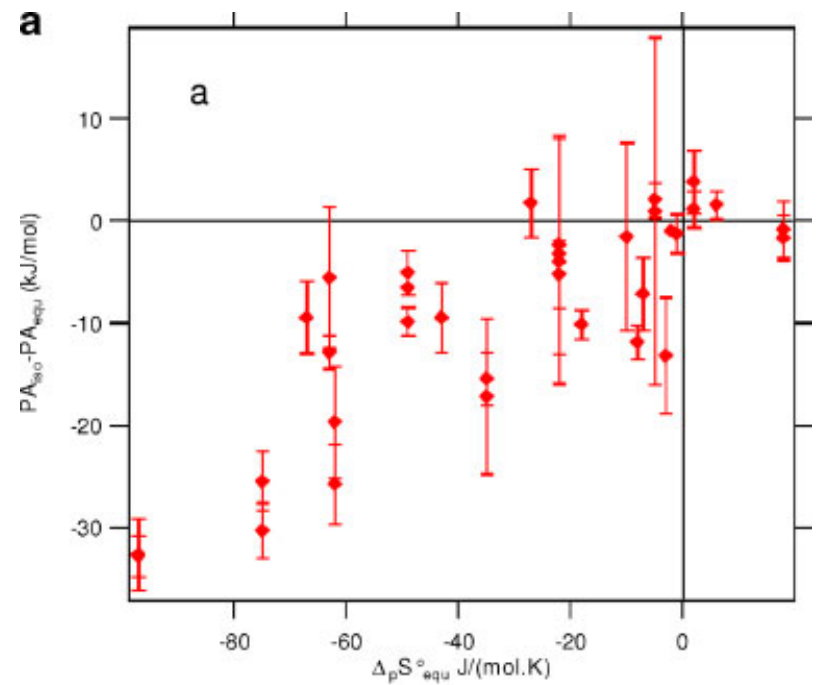

b

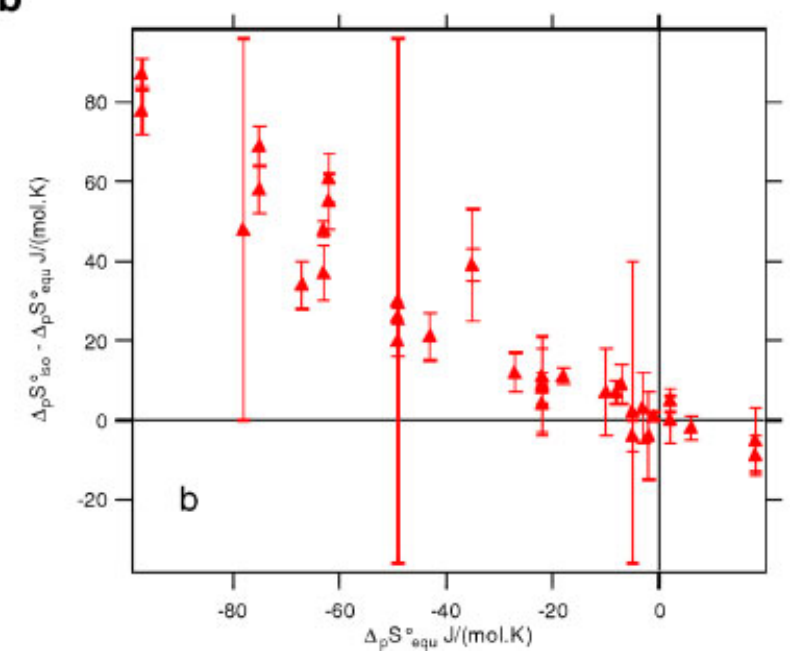

FIGURE 13. Experimental proton affinity deviation (a) and protonation entropy deviation (b) versus protonation entropy $\Delta_{p} S_{\text {equ. }}^{0}$. [Color figure can be viewed in the online issue, which is available at www.interscience. wiley.com.]

thermochemical quantities of interest, $\mathrm{PA}_{298}(\mathrm{M}), \mathrm{GB}_{298}(\mathrm{M})$ and $\Delta_{p} S^{0}(\mathrm{M})$. It may be underlined here that, since the transient intermediate $\left[\mathrm{MHB}_{i}\right]^{+}$is not necessarily in thermal equilibrium with its surroundings, the temperature $T$ may be considered as an effective temperature, exactly as with the "kinetic" method.

Using the thermokinetic method consists to deduce the gas-phase basicity $\mathrm{GB}_{298}(\mathrm{M})$ by plotting experimental $\mathrm{RE}$ values obtained for a series of reaction (if) involving bases $\mathrm{B}_{i}$ of known basicities, as a function of $\mathrm{GB}_{298}\left(\mathrm{~B}_{i}\right)$ and by fitting the data with a parametric function of the type:

$$
\mathrm{RE}=\frac{a}{1+\exp \left(b\left(c-\mathrm{GB}_{298}\left(\mathrm{~B}_{i}\right)\right)\right.}
$$

where $a$ is a normalizing factor, $b$ the slope of the curve at $\mathrm{RE}=0.5$ and $c$ is the position of the point $\mathrm{RE}=0.5$ on a GB scale. Then, comparing Equations (50) and (79), it appears that the two latter correlation parameters may be theoretically equated to 

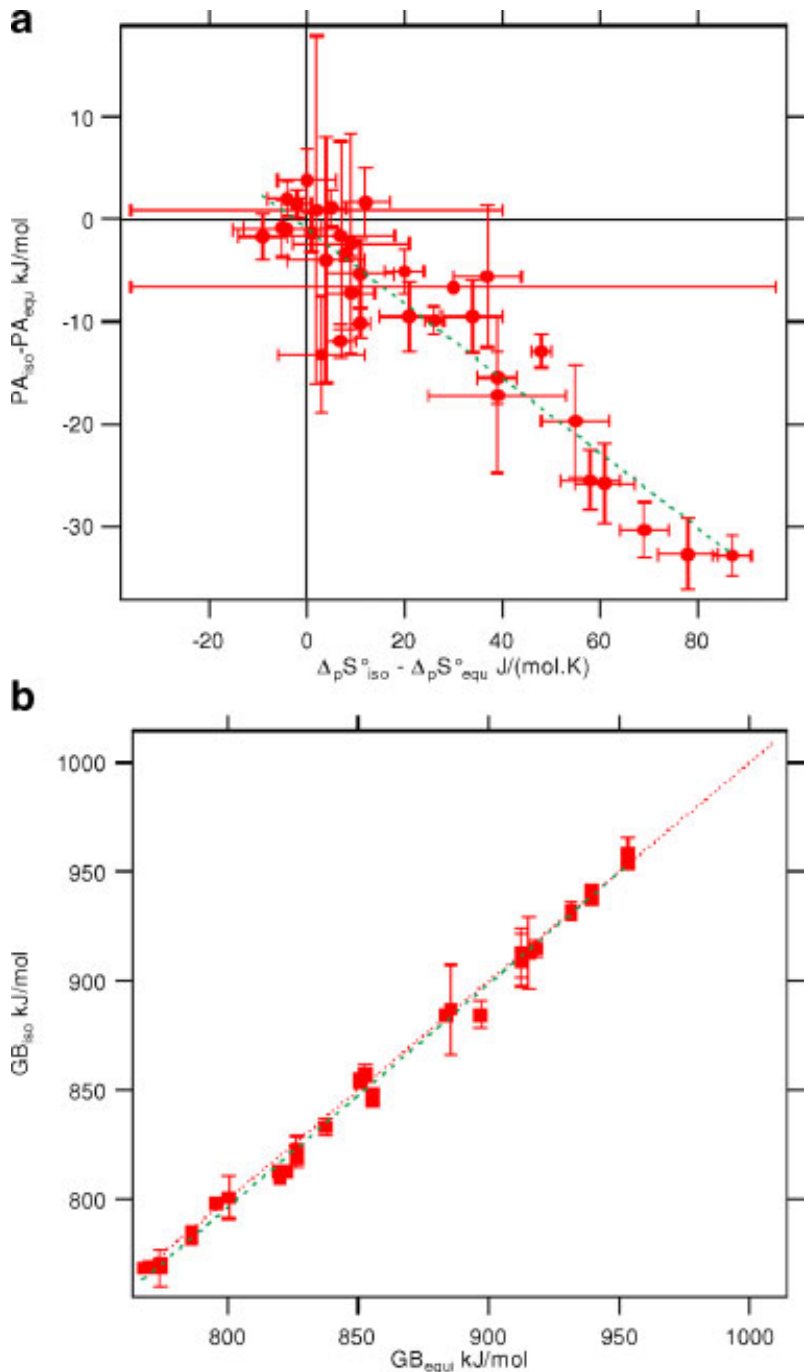

FIGURE 14. Experimental correlation between (a) enthalpy and entropy deviations and (b) gas-phase basicities obtained from the extended kinetic method as a function of its value from equilibrium method. [Color figure can be viewed in the online issue, which is available at www.interscience.wiley.com.]

$b=1 / R T$ and:

$$
c=\mathrm{GB}_{298}(\mathrm{M})-(T-298) \Delta_{i} S_{298}^{0}+\Delta_{i} H_{298 \rightarrow T}^{0}-T \Delta_{i} S_{298 \rightarrow T}^{0}
$$

In other word, when fitting the experimental RE versus $\mathrm{GB}_{298}\left(\mathrm{~B}_{i}\right)$ points by Equation $(79)$, the $\mathrm{RE}=0.5$ value corresponds to the parameter $c$, that is, to $\mathrm{GB}_{298}(\mathrm{M})$ plus a correction term $\Delta G_{\mathrm{a}}^{0}=-(T-298) \Delta_{i} S_{298}^{0}+\Delta_{i} H_{298 \rightarrow T}^{0}-T \Delta_{i} S_{298 \rightarrow T}^{0}$ (rigorously, average values over $i$ of the various terms). As discussed above, it is often assumed that the thermal corrections $\Delta H_{298 \rightarrow T}^{0}$ and $\Delta S_{298 \rightarrow T}^{0}$ may be neglected. In such circumstances, Equation (80) may be approximated by

$$
c \sim \mathrm{GB}_{298}(\mathrm{M})-(T-298)\left\langle\Delta_{i} S_{298}^{0}\right\rangle
$$

It may be noted that one may similarly consider the relationship between $\Delta_{i} G_{T}^{0}$ and the proton affinities $\mathrm{PA}_{298}\left(\mathrm{~B}_{i}\right)$ (Eq. (49)) and fit the experimental RE data by the function:

$$
\mathrm{RE}=\frac{a}{1+\exp \left(b\left(c^{\prime}-\mathrm{PA}_{298}\left(\mathrm{~B}_{i}\right)\right)\right.}
$$

Then, the $\mathrm{RE}=0.5$ value will correspond to a parameter $c^{\prime}$ equal to:

$$
c^{\prime}=\mathrm{PA}_{298}(\mathrm{M})-T\left\langle\Delta_{i} S_{298}^{0}\right\rangle+\Delta_{i} H_{298 \rightarrow T}^{0}-T \Delta_{i} S_{298 \rightarrow T}^{0}
$$

which may be simplified to:

$$
c^{\prime} \sim \mathrm{PA}_{298}(\mathrm{M})-T\left\langle\Delta_{i} S_{298}^{0}\right\rangle
$$

It is apparent from Equations (81) and (84) that if experiments are done at only one effective temperature, as typically operated, the thermokinetic method can provide $\mathrm{GB}_{298}(\mathrm{M})$ and $\mathrm{PA}_{298}(\mathrm{M})$ only if $\left\langle\Delta_{i} S_{298}^{0}\right\rangle$ is close to zero or known by another mean. A second observation is that $\mathrm{GB}_{298}(\mathrm{M})$ may be directly equated to the parameter $c$ if the effective temperature $T$ is close to $298 \mathrm{~K}$ (Eq. (81)). This may be illustrated by calculations of reaction efficiencies, RE, for model systems. As shown in Figure $15 \mathrm{a}$, on a set of reactions involving no entropy change, the sigmoid curves corresponding to the three considered temperatures intercept at $\mathrm{RE}=0.5$ for a difference in Gibbs free energy of reactant and products equal to zero. Similarly, when the effective temperature is precisely equal to $298 \mathrm{~K}$, the calculated $\mathrm{RE}$ are always equal to 0.5 when $c=\mathrm{GB}(\mathrm{M})$ whatever the entropy term $\left\langle\Delta_{i} S_{298}^{0}\right\rangle$ may be (Fig. 15b; Bouchoux, 2006a).

Practically, the thermokinetic method has been applied to rate constants determined in ion cyclotron resonance experiments (Witt \& Grutzmacher, 1997; Mormann, Salpin, \& Kuck, 1999; Chiavarino, Crestoni, \& Fornarini, 2003; Pepi, Rici, \& Rosi, 2003; Davalos et al., 2006) and in SIFT experiments (Decker, Adams, \& Babcock, 1999), mostly for basicity determinations but measurements of ionization energies (Bouchoux, Leblanc, \& Sablier, 2001), acidities (Mormann et al., 2000) and halide ion attachment (Brown et al., 2002) have been also reported.

One example of application of the thermokinetic method to the determination of the basicity of a monodentate base is illustrated by Figure 16. The gas-phase basicity of ethyl iodide tabulated in the compilation of Hunter and Lias (1998) was estimated by bracketing between that of propene and $\mathrm{H}_{2} \mathrm{~S}$. The suggested value, $\mathrm{GB}\left(\mathrm{C}_{2} \mathrm{H}_{5} \mathrm{I}\right)=698.3 \mathrm{~kJ} \mathrm{~mol}^{-1}$, was consequently attached with a considerable error of $\pm 24.5 \mathrm{~kJ} \mathrm{~mol}^{-1}$. In 2001, FT-ICR experiments involving the measurement of the deprotonation rate of protonated ethyl iodide by a set of eight bases were treated by the thermokinetic method (Bouchoux et al., 2001a). The fitting of the data leads to parameters $a, b$ and $c$ (Eq. (79)) equal to $1.00 \pm 0.04,0.30 \pm 0.05 \mathrm{~kJ}^{-1} \mathrm{~mol}$ and $689.0 \pm 0.8 \mathrm{~kJ} \mathrm{~mol}^{-1}$, respectively. Using a calculated protonation entropy of $29 \mathrm{~J} \mathrm{~mol}^{-1} \mathrm{~K}^{-1}$ for ethyl iodide and the tabulated $\Delta_{p} S^{0}\left(\mathrm{~B}_{i}\right)$ values, the mean $\left\langle\Delta_{i} S_{298}^{0}\right\rangle$ term is estimated to $-21 \mathrm{~J} \mathrm{~mol}^{-1} \mathrm{~K}^{-1}$. These results allows consequently to estimate $\mathrm{GB}\left(\mathrm{C}_{2} \mathrm{H}_{5} \mathrm{I}\right)=685.7 \pm 2.5 \mathrm{~kJ} \mathrm{~mol}^{-1}$ and $\mathrm{PA}\left(\mathrm{C}_{2} \mathrm{H}_{5} \mathrm{I}\right)=709.2 \pm$ $2.5 \mathrm{~kJ} \mathrm{~mol}^{-1}$. The later value has been confirmed by high level molecular orbital calculations, indicating that tabulated $\mathrm{PA}\left(\mathrm{C}_{2} \mathrm{H}_{5} \mathrm{I}\right)$ and $\mathrm{GB}\left(\mathrm{C}_{2} \mathrm{H}_{5} \mathrm{I}\right)$ values should be revised downward. 


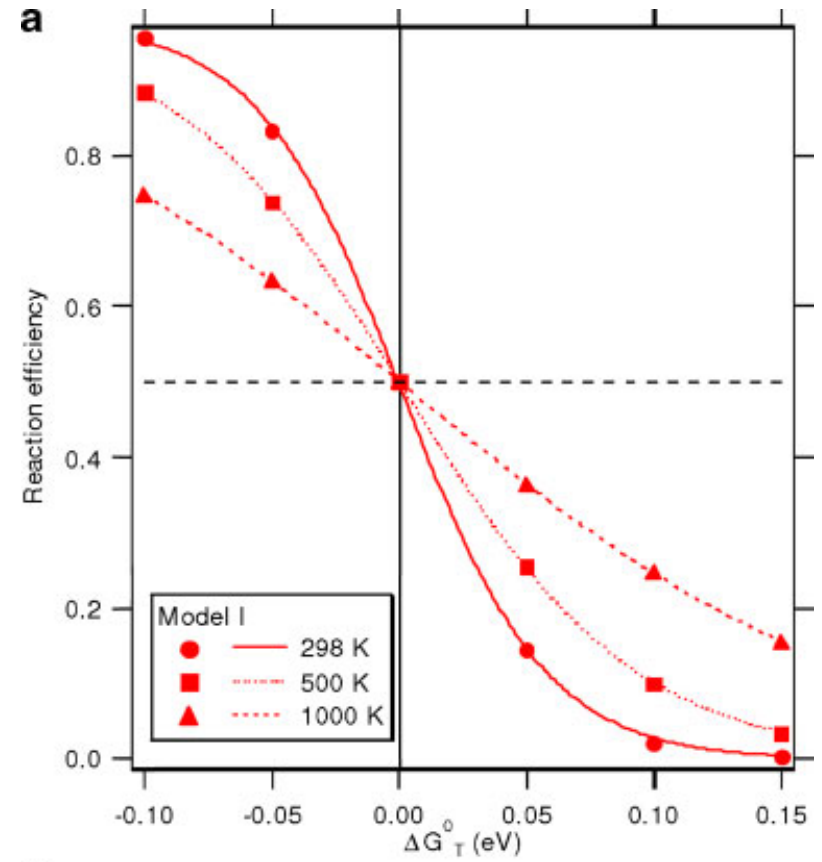

b

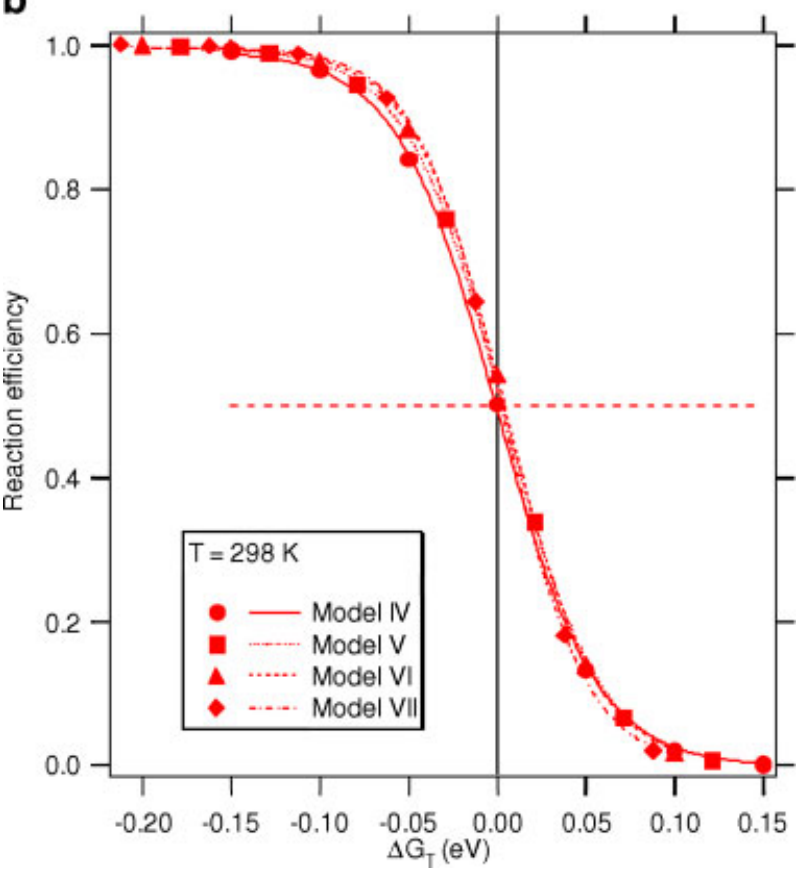

FIGURE 15. Thermokinetic plot (a) for a reaction assumed to proceed without entropy change at variable temperature of the reactants and (b) for reactions involving entropy changes in the range 0 to $34 \mathrm{~J} \mathrm{~mol}^{-1} \mathrm{~K}^{-1}$ at fixed temperature of $298 \mathrm{~K}$. [Color figure can be viewed in the online issue, which is available at www.interscience.wiley.com.]

Another favorable example, which show that the thermokinetic method may provide accurate $298 \mathrm{~K}$ gas-phase basicity if the effective temperature is in the vicinity of $298 \mathrm{~K}$, is given below for a bidentate base: the 1,3-propanediamine (Fig. 17; Bouchoux \& Buisson, 2006).

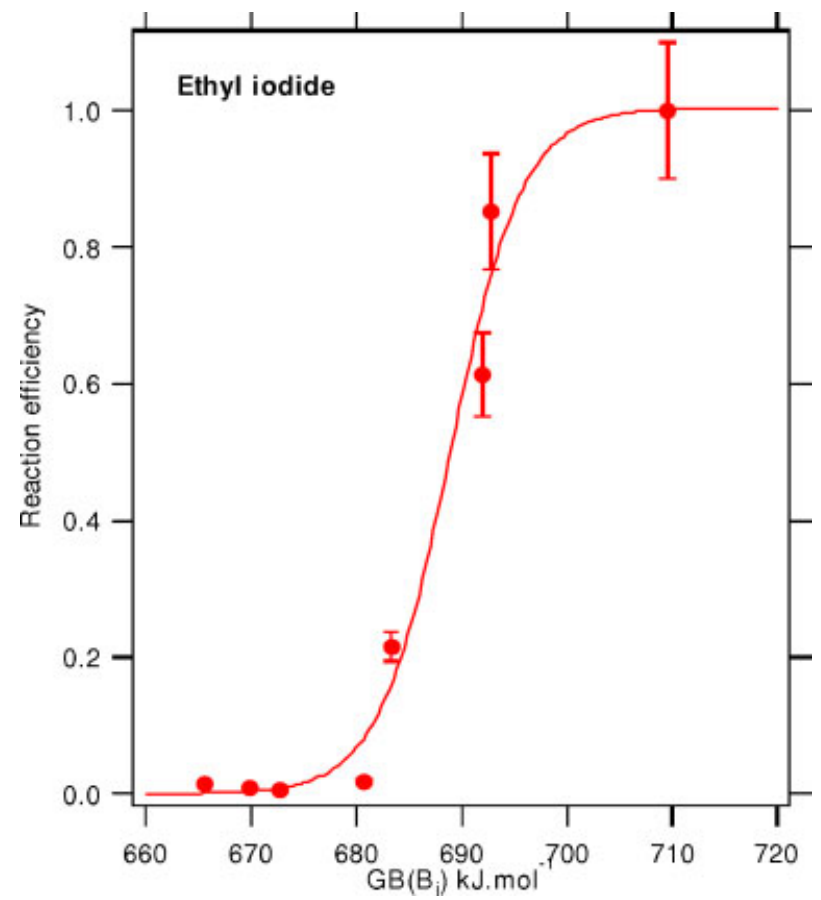

FIGURE 16. Normalized reaction efficiency as a function of $\mathrm{GB}\left(\mathrm{B}_{i}\right)$ for the deprotonation reactions of ethyl iodide: $\mathrm{C}_{2} \mathrm{H}_{5} \mathrm{IH}^{+}+\mathrm{B}_{i} \rightarrow$ $\mathrm{C}_{2} \mathrm{H}_{5} \mathrm{I}+\mathrm{B}_{i} \mathrm{H}^{+}$. [Color figure can be viewed in the online issue, which is available at www.interscience.wiley.com.]

This molecule has been extensively examined by equilibrium methods and by theory. The experimental gas-phase basicity is $939.0 \pm 2.0 \mathrm{~kJ} \mathrm{~mol}^{-1}$ and the reported protonation entropy values were situated in a very large range, between -81 and $-23 \mathrm{~J} \mathrm{~mol}^{-1} \mathrm{~K}^{-1}$. As indicated in Figure 17, the thermokinetic method applied to this molecule leads to a parameter $b$ equal to $0.3792 \mathrm{~kJ}^{-1}$ mol which corresponds to an effective temperature $T$ of $320 \mathrm{~K}$. On the other hand, the parameter $c$ is equal to $936.3 \mathrm{~kJ} \mathrm{~mol}^{-1}$ which, using Equation (81) and the tabulated $\Delta_{p} S^{0}\left(\mathrm{~B}_{i}\right)$ and $\Delta_{p} S^{0}(1,3$-propanediamine $)=-81$ or $-23 \mathrm{~J} \mathrm{~mol}^{-1} \mathrm{~K}^{-1}$ leads to a $\mathrm{GB}(1,3$-propanediol) values equal to 938.2 and $936.9 \mathrm{~kJ} \mathrm{~mol}^{-1}$. Clearly, the impact of the uncertainty on the entropic term is largely erased by fact that the effective temperature $T$ is close to $298 \mathrm{~K}$. It is important to underline the excellent agreement of the present thermokinetic gas-phase basicity with the values obtained by the equilibrium method which confirm the validity of the method. However, in situation generally observed for polyfunctional molecules, that is, when the entropy of protonation is significant and unknown, and the effective temperature different from $298 \mathrm{~K}$, the thermokinetic method should be used carefully (Bouchoux, 2006a).

In summary, the thermokinetic method used at a fixed effective temperature $T$ may give (i) $\mathrm{GB}_{298}(\mathrm{M})$ or $\mathrm{PA}_{298}(\mathrm{M})$ using Equations (81) or (84) if $\left\langle\Delta_{i} S_{298}^{0}\right\rangle$ is negligible or evaluated by another means, and (ii) $\mathrm{GB}_{298}(\mathrm{M})$ through Equation (81) if $T \sim 298 \mathrm{~K}$, even if the entropy variation of the proton transfer reaction is significant. To obtain both $\mathrm{GB}_{298}(\mathrm{M})$ (or $\mathrm{PA}_{298}(\mathrm{M})$ ) and $\left\langle\Delta_{i} S_{298}^{0}\right\rangle$, the use of the thermokinetic method at several 


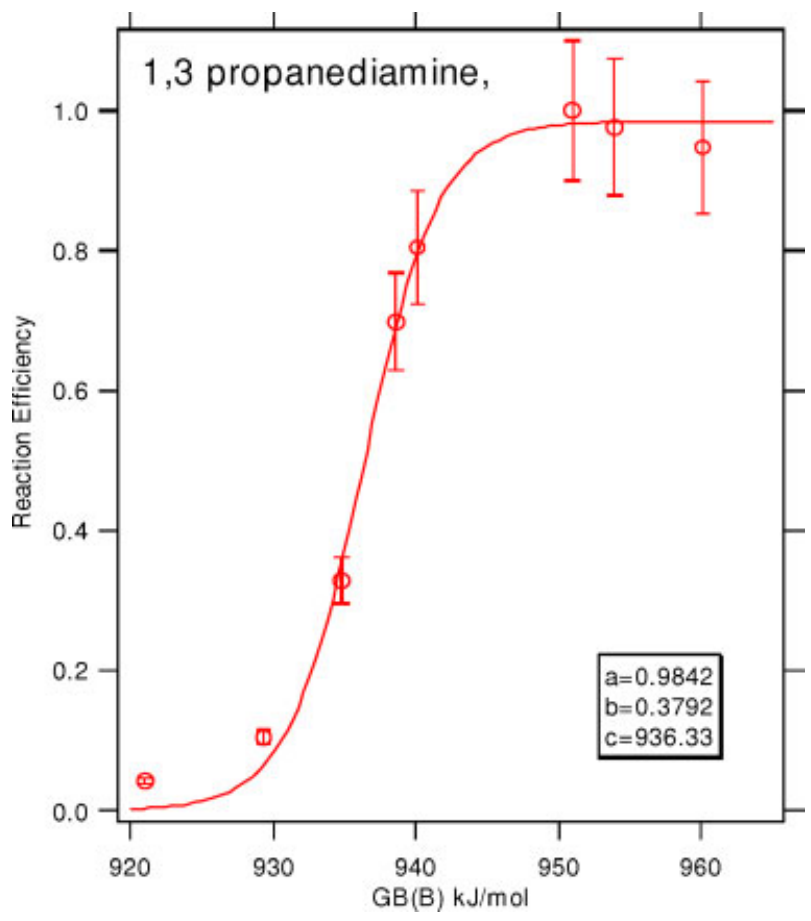

FIGURE 17. Normalized reaction efficiency as a function of $\mathrm{GB}\left(\mathrm{B}_{i}\right)$ for deprotonation reactions of 1,3-propanediamine ${ }^{+}$. [Color figure can be viewed in the online issue, which is available at www.interscience. wiley.com.]

excitation energies (i.e., effective temperatures) is necessary. This situation is comparable to that encountered with the kinetic method presented above.

\section{Theoretical Approaches}

Theoretical chemistry allows in principle the estimate of thermochemical quantities such as proton affinity and protonation entropy. Overviews of the computational methods utilized to evaluate proton affinities and gas-phase basicities have been presented recently (Irikura, 1999; Alcami, Mo, \& Yanez, 2002; Deakyne, 2003; Gordon, 2003; Yanez, 2003), only a summary will be given here. Examples which will be presented here concern only the large sets of molecules considered to test the validity of the methods, more specific theoretical results will be dispatched in the forthcoming Parts of this review.

\section{Calculation of Proton Affinity}

Molecular orbital theory or density functional theory, DFT, provide, as a first result, the potential energy $E^{0}$ also called "total energy" (in fact the potential energy of the system relative to infinitely separated electrons and nuclei) of the molecular system, generally after an iterative "geometry optimization" cycle locating the geometry of lowest $E^{0}$. Statistical thermodynamic is necessary to complete the calculation at any temperature $T$, even at $0 \mathrm{~K}$. For example, the internal energy $U_{T}$ will be obtained by adding to $E^{0}$ the $\left(U_{T}\right)$ contributions for translation, rotation, and vibration, not forgetting the zero point vibrational energy term ZPVE, according to the equations given in General:

$$
U_{T}=E^{0}+\left(U_{T}^{0}\right)_{\text {transl }}+\left(U_{T}^{0}\right)_{\mathrm{rot}}+\mathrm{ZPVE}+\left(U_{T}^{0}\right)_{\mathrm{vib}}
$$

Further, enthalpy and Gibbs free energy are obtained from the standard equations $H_{T}^{0}=U_{T}^{0}+\mathrm{PV}$ and $G_{T}^{0}=H_{T}^{0}-T S_{T}^{0}$. If we remember that the $\left(U_{T}^{0}\right)$ contributions for translation and rotation are $1 / 2 R T$ for each degree of freedom it may be written that, for a non-linear molecule:

$$
\begin{aligned}
& U_{T}^{0}=E^{0}+\mathrm{ZPVE}+\left(U_{T}^{0}\right)_{\mathrm{vib}}+3 R T \\
& H_{T}^{0}=E^{0}+\mathrm{ZPVE}+\left(U_{T}^{0}\right)_{\mathrm{vib}}+4 R T
\end{aligned}
$$

and, for a monoatomic specie:

$$
\begin{aligned}
& U_{T}^{0}=E^{0}+\frac{3}{2 R T} \\
& H_{T}^{0}=E^{0}+\frac{5}{2 R T}
\end{aligned}
$$

which, for the proton, reduces to $U_{T}^{0}=3 / 2 R T$ and $H_{T}^{0}\left(\mathrm{H}^{+}\right)=$ $5 / 2 R T$.

It is now simple to evaluate the theoretical proton affinity of a molecule $\mathrm{M}$, that is, the standard enthalpy of reaction (1). At a given temperature $T$ this quantity is given by

$$
\begin{aligned}
\mathrm{PA}_{T}(\mathrm{M}) & =\Delta_{1} H_{T}^{0}=H_{T}^{0}(\mathrm{M})+H_{T}^{0}\left(\mathrm{H}^{+}\right)-H_{T}^{0}\left(\mathrm{MH}^{+}\right) \\
& =H_{T}^{0}(\mathrm{M})-H_{T}^{0}\left(\mathrm{MH}^{+}\right)+\frac{5}{2 R T}
\end{aligned}
$$

or, if we define $\Delta X_{T}(\mathrm{M})=X_{T}(\mathrm{M})-X_{T}\left(\mathrm{MH}^{+}\right)$:

$$
\mathrm{PA}_{T}(\mathrm{M})=\Delta E^{0}(\mathrm{M})+\Delta \mathrm{ZPVE}(\mathrm{M})+\Delta U_{T}^{0}(\mathrm{M})_{\mathrm{vib}}+\frac{5}{2 R T}
$$

Reliable computation of proton affinity thus needs a high accuracy on $\Delta E^{0}(\mathrm{M})$ and on the vibrational contribution to energy $\Delta \mathrm{ZPVE}(\mathrm{M})+\Delta U_{T}^{0}(\mathrm{M})_{\mathrm{vib}}$. Quantum chemistry methods which may approach the so-called "chemical accuracy" (i.e., precision on energetic properties of ca. $\pm 5 \mathrm{~kJ} \mathrm{~mol}^{-1}$ ) are essentially "composite" and DFT methods.

a. Composite methods. The first widely used composite methods which were constructed to reproduce enthalpy of formation, ionization energy, electron affinity and proton affinity were the Gn methods (Curtiss et al., 1991, 2000, 2002 and references cited in Alcami, Mo, \& Yanez, 2001; Deakyne, 2003). In these methods, equilibrium geometry and harmonic vibrational frequencies are computed at a rather low level of theory and, using this reference geometry, single point energies are computed using more elaborated basis sets and electron correlation levels. The final energy is calculated assuming additivity of the various contributions and adjusted, to reproduce experimental data obtained along with a test set of molecules, by an empirical high level correction (HLC). The latest formulation of the Gn composites is the G3 method which was designed to correct some of the deficiencies of the G2 theory. Among the improvements, the empirical HLC term was fitted to a set of ca. 300 experimentally determined quantities and may also introduces scaled 
HLC factors or improved equilibrium geometries and vibrational frequencies (Curtiss \& Raghavachari, 2002). It may be noted that reaction (1) $\mathrm{MH}^{+} \rightarrow \mathrm{M}+\mathrm{H}^{+}$is an isogyric process, that is, a reaction where the number of electron pairs is conserved. As a corollary, the HLC terms used in the computation of the total energies of the molecule and its protonated form in the $\mathrm{Gn}$ methods exactly cancel and do not affect the quality of the proton affinity estimates using Equation (91).

Beside the Gn methods, other composite approaches have been introduced which are able to provide accurate proton affinities. Some of these notable composite methods are (i) the complete basis set (CBS-n) approach of Petersson et al. (Ochterski, Petersson, \& Montgomery, 1996), (ii) the multicoefficient correlation method of Truhlar and co-workers (Lynch \& Truhlar, 2003), (iii) the W1 and W2 methods of Martin and de Oliveira (1999), and (iv) the correlation consistent Composite Approach (ccCA) of DeYonker et al. (DeYonker et al., 2006; DeYonker, Cundari, \& Wilson, 2006). Like the Gn methods, the two former procedures are based on the inclusion of an empirical parameter to adjust the computational results to a set of experimental data. By contrast, no empirical parameter is introduced in the $\mathrm{W} 1, \mathrm{~W} 2$, and ccCA methods. The rigorous character of these approaches is based on the estimate of several contributions by extrapolation to infinite basis sets (W1, W2) or by the use of correlation consistent polarized valence basis sets (ccCA).

The vibrational frequencies calculated in all the aforementioned methods are based on the harmonic oscillator approximation. Most of the ab initio methods, from Hartree-Fock to highly correlated levels, give systematically too large fundamental frequencies. Density functional theory is subject to similar observation, though hybrid methods such as B3LYP or B3PW91/6-31G(d) seem to provide better results. In view of these drawbacks, several empirical scaling factors have been proposed in the literature (Irikura, Johnson, \& Kacker, 2005). The use of different scaling factors has been proposed to evaluate fundamental vibrational frequencies, low-frequencies vibrations or frequencies contributing to zero point energies, enthalpy and entropy corrections (Scott \& Radom, 1996).

Only eight proton affinities have been taken as experimental benchmarks in the parameterization of the Gn (Curtiss et al., 2000) and CBS-n (Ochterski, Petersson, \& Montgomery, 1996) methods, namely that of $\mathrm{NH}_{3}, \mathrm{H}_{2} \mathrm{O}, \mathrm{C}_{2} \mathrm{H}_{2}, \mathrm{SiH}_{4}, \mathrm{PH}_{3}, \mathrm{H}_{2} \mathrm{~S}, \mathrm{HCl}$, and $\mathrm{H}_{2}$. The $\mathrm{W} 1$ and $\mathrm{W} 2$ methods are shown to reproduce these experimental quantities to $\pm 2 \mathrm{~kJ} \mathrm{~mol}^{-1}$ (Parthiban \& Martin, 2001) while the absolute error attains $\pm 5 \mathrm{~kJ} \mathrm{~mol}^{-1}$ with the ccCA methods (DeYonker et al., 2006). Further, the accuracy of various composite methods has been tested on larger sets of proton affinities. Smith and Radom (1993) calculate at the G2 level the proton affinity of a total of 31 small molecules bearing up to four heavy atoms. This study revealed that $\mathrm{G} 2$ is able to predict proton affinities within $\pm 5 \mathrm{~kJ} \mathrm{~mol}^{-1}$. Moreover, the authors confirm that the proton affinity of isobutene, used as absolute standard at this period, should be adjusted downwards by $\sim 20 \mathrm{~kJ} \mathrm{~mol}^{-1}$. The same authors show that the G2 method and its variants G2MP2 and G2(MP2,SVP) lead to proton affinities with an accuracy of ca. $10 \mathrm{~kJ} \mathrm{~mol}^{-1}$ (Smith \& Radom, 1994, 1995). Proton affinities given by the G3 method has been tested on a series of 29 simple organic molecules (Hammerum, 1999; Hammerum \& Solling,
1999). A good agreement is generally observed between proton affinities calculated using Equation (91) and experiment since the maximum deviation is close to $3 \mathrm{~kJ} \mathrm{~mol}^{-1}$. G2(MP2) and CBS-Q methods lead to comparable results. It is noted however that the HLC term used in the G3 method introduces a systematic underestimate of $3.4 \mathrm{~kJ} \mathrm{~mol}^{-1}$ when the proton affinities are deduced from calculated heats of formation of $\mathrm{M}$ and $\mathrm{MH}^{+}$. Recently, the proton affinities of a number of five-membered ring heterocycles have been derived from G3 or G3B3calculations (Kabli et al., 2006; Rao \& Sastry, 2006; van Beleen et al., 2004). Both methods give essentially the same results, moreover an excellent agreement is observed with experiment. As an illustration, Figure 18 shows the correlation observed between these $\mathrm{G} 3$ calculated proton affinities and the experimental values (Hunter \& Lias, 1998). The correlation coefficient of the fitting line is excellent, $r=0.996$, the mean value and the standard deviation of the residual $\left(-2.4\right.$ and $4.2 \mathrm{~kJ} \mathrm{~mol}^{-1}$, respectively) are of the same order as the experimental error.

It must be emphasized that theoretical calculation may also provide local proton affinities which, by comparison with the experimental value, allow the determination of the favored protonation site. For example in the heterocyclic series discussed above (Kabli et al., 2006; Rao \& Sastry, 2006), the G3 calculations demonstrate that the favored site of protonation is a carbon when there is only one heteroatom in the ring, and nitrogen when there are two or more heteroatoms. Moreover, in certain cases, calculation predicts ring opening This is for example expected when protonating $1 \mathrm{H}-1,2,3$-triazole in positions 1 or 4 (Kabli et al., 2006) or cyclic anhydrides (Bouchoux et al., 1998).

b. Density functional theory. DFT procedures offer practical alternative to composite procedures to a lower cost in computation time and in memory requirement. The choice of

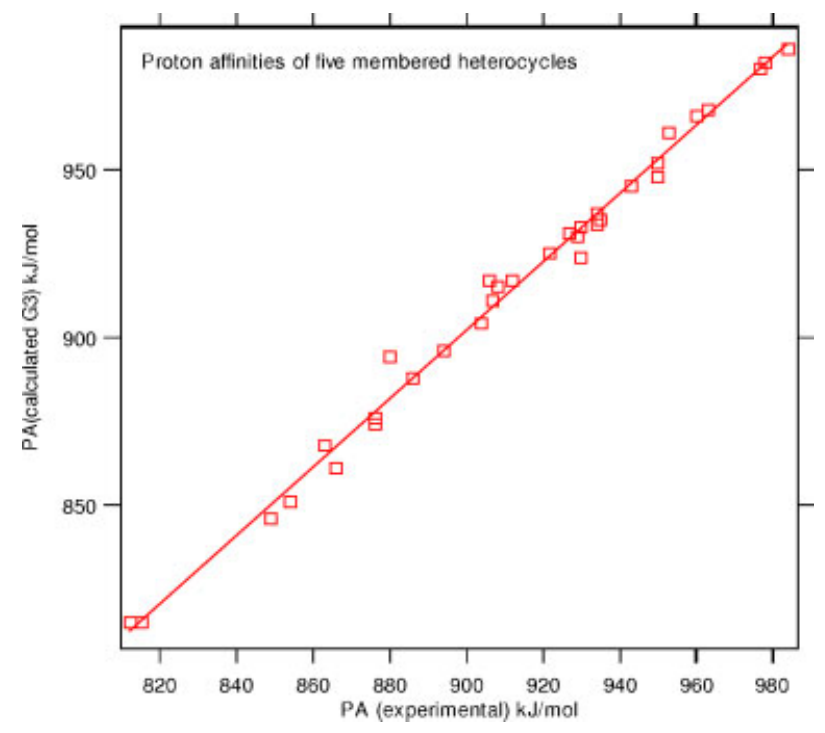

FIGURE 18. Correlation of calculated and experimental proton affinities (G3 and G3B3 calculations from Kabli et al., 2006 and Rao \& Sastry, 2006, experiment from Hunter \& Lias, 1998). [Color figure can be viewed in the online issue, which is available at www.interscience. wiley.com.] 
the exchange and correlation functionals and of the basis set size is however crucial. The most popular B3 hybrid exchange and LYP correlation functional combination appears to reproduce proton affinities to within $5 \mathrm{~kJ} \mathrm{~mol}^{-1}$ when using the $6-311+\mathrm{G}(\mathrm{d}, \mathrm{p})$ basis set (Burk et al., 2000) or the 6-311++G(d,p) basis set (Range et al., 2006; Rao \& Sastry, 2006). However, the latter authors note that, for the set of molecules they examined, the best performance was obtained for the functionals B1B95 and MPW1B95 which both use the Becke's B95 correlation functional (Range et al., 2006).

c. Other methods. A number of calculations of proton affinities (but also of other thermochemical properties) are based on the use of isodesmic reactions in which a calculated reaction enthalpy is combined with experimental enthalpy of a suitable reference reaction. Isodesmic reactions are formal reactions that conserve the number and the types of chemical bonds. It is expected that, in the calculated reaction enthalpy, the computational errors arising from deficiencies in the basis set and in the electron correlation, that are specific of a particular bond type, will cancel on both sides of the chemical equation. The advantage of this type of reaction is obviously to obtain satisfactory enthalpy from calculations done at a rather modest level of theory. Proton transfer reaction (i) $\mathrm{MH}^{+}+\mathrm{B}_{i} \rightarrow \mathrm{M}+\mathrm{BH}^{+}$pertain to this category of reactions provided the protonated sites are identical in $\mathrm{MH}^{+}$and $\mathrm{BH}^{+}$. Estimate of the proton affinity of an unknown $\mathrm{M}, \mathrm{PA}(\mathrm{M})=\mathrm{PA}\left(\mathrm{B}_{i}\right)+\Delta_{i} H^{0}$, may be done by combining a computed $\Delta_{i} H^{0}$ value with an experimental value of $\operatorname{PA}\left(\mathrm{B}_{i}\right)$. The results are generally better when using reactions where the number of atoms in a given hybridization state are the same in both sides of the reaction (Irikura, 1999).

Finally, a proton affinity may also be calculated using the standard heats of formations of reactant and products of reaction (1): $\mathrm{PA}_{298}(\mathrm{M})=\Delta_{1} H_{298}^{0}=\Delta_{\mathrm{f}} H_{298}^{0}(\mathrm{M})+\Delta_{\mathrm{f}} H_{298}^{0}\left(\mathrm{H}^{+}\right)-$ $\Delta_{\mathrm{f}} H_{298}^{0}\left(\mathrm{MH}^{+}\right)$(Eq. (3)). To obtain individual heats of formation, a recommended procedure is to calculate heats of formation at $0 \mathrm{~K}$, for example from a $\mathrm{Gn}$ type total energy, in conjunction with experimental gas-phase heats of formation of individual atoms. Then, the correction to give $298 \mathrm{~K}$ value is obtained from theory for the specie of interest and from experiment for the constituent elements (Nicolaides et al., 1996; Hammerum, 1997).

\section{Calculation of Protonation Entropy, $\Delta_{p} S^{O}$}

As discussed in General, the entropy of a particular species can be separated into electronic, translational, rotational and vibrational components and consequently the protonation entropy $\Delta_{p} S^{0}(\mathrm{M})=S^{0}\left(\mathrm{MH}^{+}\right)-S^{0}(\mathrm{M})($ Eq. (5)) may be expressed by the sum:

$$
\Delta_{p} S^{0}=\Delta_{p} S_{\mathrm{el}}^{0}+\Delta_{p} S_{\mathrm{trans}}^{0}+\Delta_{p} S_{\mathrm{rot}}^{0}+\Delta_{p} S_{\mathrm{vib}}^{0}
$$

The first term, $\Delta_{p} S_{\mathrm{el}}^{00}=S_{\mathrm{el}}^{0}\left(\mathrm{MH}^{+}\right)-S_{\mathrm{el}}^{0}(\mathrm{M})$, is generally equal to zero since the base $\mathrm{M}$ and its protonated form $\mathrm{MH}^{+}$are even electron species in their fundamental singlet electronic states.

The term $\Delta_{p} S_{\text {trans }}^{0}=S_{\text {trans }}^{0}\left(\mathrm{MH}^{+}\right)-S_{\text {trans }}^{0}(\mathrm{M})$, which following Equation (21) is equal to $(3 / 2) R \ln \left(m_{\mathrm{MH}} / m_{\mathrm{M}}\right)$, is also negligible for a molecule containing several heavy atoms, it represents less than $1 \mathrm{~J} \mathrm{~mol}^{-1} \mathrm{~K}^{-1}$ when the mass of the molecule is larger than $12 \mathrm{u}$.
The term $\Delta_{p} S_{\text {rot }}^{0}=S_{\text {rot }}^{0}\left(\mathrm{MH}^{+}\right)-S_{\text {rot }}^{0}(\mathrm{M})$ concerns the overall rotations of $\mathrm{M}$ and $\mathrm{MH}^{+}$. According to Equation (26), it depends on the product of the principal moments of inertia $D=I_{x} I_{y} I_{z}$ and on the symmetry number $\sigma$ of each species:

$$
\Delta_{p} S_{\mathrm{rot}}^{0}=\frac{1}{2} R \ln \left(\frac{D_{\mathrm{MH}}}{D_{\mathrm{M}}}\right)-R \ln \left(\frac{\sigma_{\mathrm{MH}}}{\sigma_{\mathrm{M}}}\right)
$$

Often, if the molecule is large enough, the change in moment of inertia may be neglected and $\Delta_{p} S_{\text {rot }}^{0}$ reduces to the symmetry correction $-R \ln \left(\sigma_{\mathrm{MH}} / \sigma_{\mathrm{M}}\right)$.

The vibrational contribution $\Delta_{p} S_{\mathrm{vib}}^{0}=S_{\mathrm{vib}}^{0}\left(\mathrm{MH}^{+}\right)-S_{\mathrm{vib}}^{0}(\mathrm{M})$ constitutes the most delicate and the most important point to consider. First of all, the protonated structure $\mathrm{MH}^{+}$presents three internal degrees of freedom more than $\mathrm{M}$, which contributes to $\Delta_{p} S_{\mathrm{vib}}^{0}$. If we consider only this perturbation, protonation of a basic center, said $\mathrm{X}$, is expected to generate three new vibrational modes: one $\mathrm{X}-\mathrm{H}$ valence vibration and two deformations involving bond angle and dihedral angle. As discussed in General, only low frequencies contribute significantly to the overall entropy (a frequency higher than $1,000 \mathrm{~cm}^{-1}$ corresponds to a $S^{0}$ less than $0.5 \mathrm{~J} \mathrm{~mol}^{-1} \mathrm{~K}^{-1}$ ) thus only the two deformations are expected to participate to an increase of $\Delta_{p} S_{\mathrm{vib}}^{0}$. This participation is rather limited and does not generally exceed $10 \mathrm{~J} \mathrm{~mol}^{-1} \mathrm{~K}^{-1}$. This kind of behavior is generally presented by monofunctional base where the perturbation brought by protonation is only local.

The situation may be significantly changed when the molecule presents more than one basic site since protonation may induce drastic structural changes between $\mathrm{M}$ and $\mathrm{MH}^{+}$. As a consequence, in addition to the three new internal degrees of freedom, several other modes may be also affected and so the corresponding entropy term $\Delta_{p} S_{\mathrm{vib}}^{0}$. Most of the time the source of entropy change during protonation of a polyfunctional molecule is the formation of an intramolecular $\mathrm{H}$ bond or, in the contrary, the opening of a cyclic part of the molecule. The vibrational modes associated with these structural changes are hindered internal rotations and ring deformations. The theoretical treatment of these peculiar, low frequency, vibrations has been commented in General and the difficulties to treat accurately systems containing a number of coupled internal rotations have been underlined. The approximation usually considered in the practical evaluation of the protonation entropy of polyfunctional molecules is to treat each internal rotation as a symmetrical rotor with a potential energy function given by Equation (34) (see General). Theoretical calculation of torsional potentials $V(\phi)$ can be obtained by quantum calculation at various levels of sophistications. In the simplest approach, after a total geometry optimization, the angle $\phi$ is varied while the remaining internal coordinates are kept frozen. This "rigid rotation approach" gives generally poor results particularly in the case of polyfunctional molecules. The results are significantly improved in the "relaxed rotation approach," where the internal coordinates are all optimized for each value of $\phi$. Finally, a more sophisticated method, the "reaction path approach," consists in locating exactly the transition structures connecting the various rotamers. Despite several shortcomings which may be identified (Cioslowsky, Scott, \& Radom, 1997) the relaxed rotation approach offers a good compromise between accuracy and computation time for 
large molecules. According to the $V_{0}$ value as compared to RT, several approximations may be done (refer to Figs. $2 \mathrm{~b}$ and 3, part A). If $V_{0}$ is less than $\sim 2 R T$, the rotation may be considered as free and $S^{0}$ evaluated by Equations (38) and (39). At the opposite if $V_{0}$ is larger than $\sim 20 R T S^{0}$ may be calculated within the harmonic oscillator approximation using the frequency given by Equation (35). Between these two extreme situations, the entropy of the hindered rotor should be calculated exactly or evaluated from tabulated models (Pitzer \& Gwinn, 1942) or using analytical approximations (Truhlar, 1991; McClurg, Flagan, \& Goddard, 1997; Barker \& Shovlin, 2004).

As a first approximation, East and Radom (1997) suggest to treat each rotor with $V_{0}<1.4 R T$ as free and the other as harmonic oscillators (procedure named E1 by the authors). In the E2 method proposed by East and Radom each internal rotation is treated as hindered with a simple cosine potential calculated, at the MP2/6-311 + G(2df,p)//MP2/6-31G(d) level of theory, using the relaxed rotation mode. Finally, the E3 method introduces a rotor-rotor coupling by calculating the true, two-dimensional, potential energy surface. These three levels of sophistication were applied to evaluate the protonation entropy $\Delta_{p} S^{0}(\mathrm{M})$ of set of 21 small monofunctional bases containing zero, one or two internal rotations (East, Smith, \& Radom, 1997). By comparison with experimental $\Delta_{p} S^{0}$ values (ranging from -5 to $40 \mathrm{~J} \mathrm{~mol}^{-1} \mathrm{~K}^{-1}$ ), the $\mathrm{E} 3$ procedure leads to protonation entropies accurate to approximaterly $5 \mathrm{~J} \mathrm{~mol}^{-1} \mathrm{~K}^{-1}$ (standard deviation, with a max deviation $\sim 15 \mathrm{~J}$ ). It is also noted by Radom and co-workers that the accuracy on $\Delta_{p} S^{0}$ is only reduced by $\sim 1 \mathrm{~J} \mathrm{~mol}^{-1} \mathrm{~K}^{-1}$ by using the simpler E1 or E2 procedures (Table 2).

Application of a similar model to polyfunctional molecules in which more than one rotor is altered by the protonation process has been done for diols (Bouchoux \& Berruyer-Penaud, 2003c), diamines (Bouchoux, Choret, \& Berruyer-Penaud, 2001c), aminoalcohols (Bouchoux et al., 2002, 2005) and diketones (Akrour et al., 2007). In these approaches, each internal rotation is treated as hindered rotation with a simple cosine potential, averaged if minor barriers appear in the torsional potential energy profile, if necessary. All the other vibrational modes are considered as harmonic oscillators. The method appears to reproduce a large part of the protonation entropy but significant deviations are also observed when compared with experimental values (Table 3). Part of the discrepancies may be due to an underestimate of the entropy of mixing, particularly for the neutral molecule. This is suggested by the excellent agreement observed between theory and experiment for 2,5-hexanedione where the contributions of several conformers of the neutral molecule were introduced in the calculation (Table 3).

As underlined at the end of the paragraph A.2, new methods of calculation of the entropy of molecules containing several internal rotations are in development and remain to be applied to the protonation entropy problem.

It may be also emphasized that the comparison with experiment is generally delicate for large protonation entropy values since the experimental error is, most of the time, unknown and probably not negligible.

Finally, it has been established by theoretical calculations of the rotational barriers $V_{0}$ associated with internal rotations in aliphatic bifunctional molecules that $V_{0}$ are rarely less than
$10 \mathrm{~kJ} \mathrm{~mol}^{-1}$ in the neutral molecules $\mathrm{M}$ and are generally lower than $100 \mathrm{~kJ} \mathrm{~mol}^{-1}$ in their protonated forms $\mathrm{MH}^{+}$. The entropy loss corresponding to this change in barrier height does not exceed $-15 \mathrm{~J} \mathrm{~mol}^{-1} \mathrm{~K}^{-1}$ (see Fig. 3). This may provide an approximation for the estimate of an upper limit to the corresponding entropy of protonation when no other information is available.

\section{STRUCTURAL AND ENERGETIC ASPECTS OF THE PROTONATION}

By definition, the proton affinity reflects the difference in enthalpy between a molecule $\mathrm{M}$ plus a proton and the protonated form $\mathrm{MH}^{+}$(Eq. (1)). Experimental values of proton affinities refer to the most stable forms of $\mathrm{M}$ and $\mathrm{MH}^{+}$present in the experimental conditions. It is consequently necessary, when discussing proton affinity values, to consider the factors that stabilize both the neutral molecule $\mathrm{M}$ and the positively charged species $\mathrm{MH}^{+}$. Moreover, since proton affinities are molar quantities, possibilities of equilibrium mixtures of conformers should be taken into account. Discussion concerning the gasphase basicity must, in addition, include entropy terms in particular by their molecule dependent part, that is, the so-called protonation entropy, $\Delta_{p} S^{0}(\mathrm{M})=S^{0}\left(\mathrm{MH}^{+}\right)-S^{0}(\mathrm{M})$.

The consequences of the protonation process on both structure and thermochemistry, depend on characteristics related to the nature of the proton acceptor (non-bonding or $\pi$-bonding basic electrons pair, electronegativity of the concerned atoms) and on factors related with the remaining part of the molecule such as substituent effects in a general sense (electrostatic effects including polarizability, permanent polarization, in particular intramolecular hydrogen bond, and resonance effects) and on the possibility of interfunctional interactions. The following section will present the range of gas-phase basicities and proton affinities of the most common organic molecules (Gas-Phase Basicity Scale) and the main factors that influence the stability of neutral and ionized molecular systems and their consequences on the protonation energetics: types of protonation sites and substituent effects (Characteristics Related to the Nature of the Basic Site), hydrogen bonding (Hydrogen Bonding and Protonation). A last paragraph (Covalent Bonding and Protonation) is devoted to a rapid survey of the possible consequences of interfunctional interactions in protonated clusters (necessary intermediates during the measurement of gas-phase basicity) or in protonated polyfunctional molecules.

\section{A. Gas-Phase Basicity Scale}

\section{The Absolute Scale of Gas-Phase Basicities and Proton Affinities}

The study of proton transfer reactions by the experimental methods presented in Background provides relative gas-phase basicities or proton affinities. To obtain absolute values of these quantities, anchoring to some references data is indispensable. Absolute proton affinity scale has been constructed 
TABLE 2. Comparison of experimental and theoretical protonation entropies $\left(\mathrm{J} \mathrm{mol}^{-1} \mathrm{~K}^{-1}\right)$ of some monofunctional bases ${ }^{\mathrm{a}}$

\begin{tabular}{|c|c|c|c|c|c|}
\hline \multirow[b]{2}{*}{ Base } & \multicolumn{2}{|c|}{ experimental $\Delta_{p} \mathrm{~S}^{\circ}$} & \multicolumn{3}{|c|}{ theoretical $\Delta_{\nu} \mathrm{S}^{\circ \mathrm{c}}$} \\
\hline & $600 \mathrm{~K}^{\mathrm{a}}$ & $500 \mathrm{~K}^{\mathrm{b}}$ & $600 \mathrm{~K}$ & $500 \mathrm{~K}$ & $298 \mathrm{~K}$ \\
\hline dimethylamine & -2.5 & -6.3 & 0.5 & -0.6 & -2.4 \\
\hline ethylamine & -6.7 & 2.1 & -2.3 & - & -5.4 \\
\hline methylamine & -9.6 & -12.5 & -3.5 & -4.7 & -6.6 \\
\hline pyrrole & 4.6 & 4.2 & 11.6 & 10.4 & 8.8 \\
\hline $\mathrm{NH}_{3}$ & -5.4 & -6.3 & -6.0 & -5.3 & -6.4 \\
\hline $\mathrm{CH}_{3} \mathrm{COOCH}_{3}$ & 15.4 & 16.7 & - & - & - \\
\hline acetone & 10.2 & 18.8 & 14.0 & 12.1 & 8.7 \\
\hline isobutene & 13.3 & 23.0 & 16.3 & 15.7 & 16.2 \\
\hline $\mathrm{C}_{2} \mathrm{H}_{5} \mathrm{CN}$ & 12.1 & 10.5 & 14.0 & 11.5 & 5.6 \\
\hline $\mathrm{CH}_{3} \mathrm{OCH}_{3}$ & 23.0 & 18.8 & 22.7 & 20.8 & 16.5 \\
\hline $\mathrm{HCOOCH}_{3}$ & 17.3 & 2.1 & 11.4 & 9.3 & 5.1 \\
\hline toluene & 24.0 & 12.6 & 18.8 & - & 11.3 \\
\hline $\mathrm{CH}_{3} \mathrm{CN}$ & 12.7 & - & 12.6 & 10.0 & 4.3 \\
\hline $\mathrm{CH}_{3} \mathrm{OH}$ & 22.6 & -2.1 & 14.8 & 12.5 & 7.3 \\
\hline benzene & 30.1 & 27.2 & 33.5 & - & 26.3 \\
\hline $\mathrm{CH}_{3} \mathrm{CHO}$ & 15.3 & - & 7.8 & 5.7 & 1.3 \\
\hline propene & 27.6 & 8.4 & 15.5 & 13.8 & 10.2 \\
\hline $\mathrm{H}_{2} \mathrm{~S}$ & - & 6.3 & 7.1 & 5.6 & 3.4 \\
\hline $\mathrm{H}_{2} \mathrm{O}$ & - & 6.3 & 8.7 & 7.2 & 4.8 \\
\hline ethylene & - & 16.7 & 18.0 & 16.0 & 12.2 \\
\hline $\mathrm{CO}$ & $\cdot$ & 12.6 & 9.5 & 7.5 & 3.8 \\
\hline $\mathrm{CO}_{2}$ & $\cdot$ & 33.5 & 33.0 & 31.1 & 26.4 \\
\hline $\mathrm{CS}_{2}$ & - & 46.0 & 37.6 & 35.7 & 31.3 \\
\hline $\cos$ & - & 31.4 & 33.7 & 31.7 & 26.6 \\
\hline $\mathrm{CH}_{4}$ & - & 33.5 & 33.5 & - & 26.4 \\
\hline $\mathrm{N}_{2}$ & - & 20.9 & 17.7 & 15.4 & 10.5 \\
\hline
\end{tabular}

${ }^{\mathrm{a}}$ Mautner (2003).

${ }^{\mathrm{b}}$ Szulejko and McMahon (1993).

${ }^{\mathrm{c}}$ East, Smith, and Radom (1997).

using Equation (3) $\left[\mathrm{PA}_{298}(\mathrm{M})=\Delta_{\mathrm{f}} H_{298}^{0}(\mathrm{M})+\Delta_{\mathrm{f}} H_{298}^{0}\left(\mathrm{H}^{+}\right)-\right.$ $\left.\Delta_{\mathrm{f}} H_{298}^{0}\left(\mathrm{MH}^{+}\right)\right]$for a set of reference molecules $\mathrm{M}$ of which heats of formation of the neutral and protonated forms were accurately known. Most of the time the latter term, $\Delta_{\mathrm{f}} H_{298}^{0}\left(\mathrm{MH}^{+}\right)$, is coming from the determination of a suitable appearance energy of the considered ion by a mass spectrometry technique (for a review of the methods see Ervin, 2000). A first database of proton affinities and gas-phase basicities, anchored to a number of absolute reference bases including ammonia, ketene, isobutene, propylene, formaldehyde, water, ethylene, carbon monoxide, carbon dioxide, oxygen atom and molecular oxygen, was produced in 1984 (Lias, Liebman, \& Levin, 1984). In the following years, Mautner and Sieck (Mautner \& Sieck, 1991a,b; Sieck, 1997) on the one hand, Szulejko and McMahon (1993) on the other, present two thermochemical ladders based on the measurement of equilibrium constants at variable temperature in a high pressure ion source for a set of overlapping proton transfer reactions. These authors show, inter alia, that the proton affinity of isobutene, one of the standards in the 1984 database, was in error by $\sim 17 \mathrm{~kJ} \mathrm{~mol}^{-1}$. The evaluation procedure used in the most recent compilation of protonation thermochemical data (Hunter \& Lias, 1998, 2005) takes into account these two ladders and introduce temperature corrections on several sets of experiments. More recent threshold appearance energy measurements and theoretical calculations of protonation entropies and proton affinities (Smith \& Radom, 1993) are also taken into account. The thermochemical data of the primary standard considered in this compilation are reported in Table 4 . Since a long time, ammonia has been used as a reference base in a large number of studies, this traditional standard is also listed in Table 4. The data discussed in 
TABLE 3. Comparison of experimental and theoretical protonation entropies of some bifunctional bases

\begin{tabular}{ccc}
\hline Base & experimental $\Delta_{p} \mathbf{S}^{\circ}(500-600 \mathrm{~K})$ & theoretical $\Delta_{p} \mathbf{S}^{\circ}(300 \mathrm{~K})$ \\
\hline 1,2-diaminoethane & $-22^{\mathrm{a}}$ & $-17^{\mathrm{d}}$ \\
1,3-diaminopropane & $-49^{\mathrm{a}}$ & $-29^{\mathrm{d}}$ \\
1,4-diaminobutane & $-63^{\mathrm{a}}$ & $-46^{\mathrm{d}}$ \\
4-hydroxy-2-butanone & $-45^{\mathrm{b}}$ & $-20^{\mathrm{b}}$ \\
methoxyacetone & $-18^{\mathrm{b}}$ & $-6^{\mathrm{b}}$ \\
4-methoxybutanone & $-37^{\mathrm{b}}$ & $-26^{\mathrm{b}}$ \\
5-methoxy-2-pentanone & $-48^{\mathrm{b}}$ & $-43^{\mathrm{b}}$ \\
2,5-hexanedione & $-27^{\mathrm{c}}$ & $-25^{\mathrm{c}, \mathrm{c}}$ \\
& & $-33^{\mathrm{c}, \mathrm{f}}$ \\
\hline
\end{tabular}

\footnotetext{
${ }^{\mathrm{a}}$ Mautner et al. (1980).

${ }^{\mathrm{b}}$ Bouchoux et al. (2005).

${ }^{\mathrm{c}}$ Akrour et al. (2007).

${ }^{\mathrm{d} B o u c h o u x}$, Choret, and Berruyer-Penaud (2001c).

${ }^{\mathrm{e}}$ Entropy calculation including the entropy of mixing and assuming formation of an internal ionic hydrogen bond in $\mathrm{MH}^{+}$.

${ }^{\mathrm{f}}$ Entropy calculation including the entropy of mixing and assuming cyclization to form $\mathrm{MH}^{+}$ions of structure: c- $\left(\mathrm{CH}_{3}\right) \mathrm{COC}\left(\mathrm{OH}, \mathrm{CH}_{3}\right) \mathrm{CH}_{2} \mathrm{CH}_{2}$.
}

the present review were, when necessary, corrected to this proton affinity/gas-phase basicity scale.

Recently, Mautner (2003) proposes slight readjustments of these proton affinity and gas-phase basicity scales by carefully reconsidering possible sources of error in the measurements obtained from low pressure ion cyclotron resonance mass spectrometry and pulsed high-pressure mass spectrometry. Most of the discrepancies are originating from uncertainties in temperature measurement which leads to low $(<10 \%)$ but systematic expansion or contractions of the energy scale. A global adjustment for these effects is done by using adequate scaling factors which provide proton affinities and gas-phase basicities with a residual error of ca. $3 \mathrm{~kJ} \mathrm{~mol}^{-1}$.

\section{Overview of the Data}

The present proton affinity database (Hunter \& Lias, 1998, 2005) extends from ca. 180 to $1,400 \mathrm{~kJ} \mathrm{~mol}^{-1}$. It starts from rare or inert gases: $\mathrm{He}\left(\mathrm{PA}=178 \mathrm{~kJ} \mathrm{~mol}^{-1}\right), \mathrm{Ne}\left(\mathrm{PA}=199 \mathrm{~kJ} \mathrm{~mol}^{-1}\right)$, $\operatorname{Ar}\left(\mathrm{PA}=369 \mathrm{~kJ} \mathrm{~mol}^{-1}\right), \mathrm{H}_{2}\left(\mathrm{PA}=422 \mathrm{~kJ} \mathrm{~mol}^{-1}\right), \mathrm{Kr}(\mathrm{PA}=$ $\left.425 \mathrm{~kJ} \mathrm{~mol}^{-1}\right), \mathrm{N}_{2}\left(\mathrm{PA}=494 \mathrm{~kJ} \mathrm{~mol}^{-1}\right), \mathrm{Xe}\left(\mathrm{PA}=499 \mathrm{~kJ} \mathrm{~mol}^{-1}\right)$, and extends up to metal oxides: $\mathrm{Na}_{2} \mathrm{O}\left(\mathrm{PA}=1,376 \mathrm{~kJ} \mathrm{~mol}^{-1}\right)$, $\mathrm{Cs}_{2} \mathrm{O}\left(\mathrm{PA}=1,443 \mathrm{~kJ} \mathrm{~mol}^{-1}\right)$. Most of the organic compounds fall within a more restricted range, extending from ca. 600 to $1,100 \mathrm{~kJ} \mathrm{~mol}^{-1}$. An illustration of this part of the proton affinity scale is presented in Figure 19. Emphasis is given in this Figure to the most common monofunctional bases containing as heavy atoms: carbon, halogens, oxygen, nitrogen, sulfur, and phosphorus. To complete this correlation chart, the gas-phase basicity and proton affinity of a selection of representative organic molecules is given in Table 5 .

A rapid examination of Figure 19 shows clearly the progressive shift of proton affinity toward large values when the electronegativity of the atoms responsible of the proton attachment increases. Roughly, halides are less basic than oxygenated compound, themselves less basic than nitrogen containing molecules. The low basicity of alkanes was also expected, note however that the $540-680 \mathrm{~kJ} \mathrm{~mol}^{-1}$ range corresponds to the only four reported PA values of methane $\left(543 \mathrm{~kJ} \mathrm{~mol}^{-1}\right)$, ethane $\left(596 \mathrm{~kJ} \mathrm{~mol}^{-1}\right)$, propane $\left(626 \mathrm{~kJ} \mathrm{~mol}^{-1}\right)$, and isobutane $\left(678 \mathrm{~kJ} \mathrm{~mol}^{-1}\right)$. The aromatic range extends from benzene $\left(\mathrm{PA}=750 \mathrm{~kJ} \mathrm{~mol}^{-1}\right)$ to azulene $\left(\mathrm{PA}=930 \mathrm{~kJ} \mathrm{~mol}^{-1}\right)$.

As underlined above, Figure 19 contains only data relevant to $\mathrm{C}, \mathrm{O}, \mathrm{N}, \mathrm{S}, \mathrm{P}$, and halogen monofunctional molecules. It may be indicated that polyfunctional organic bases fall also in the $600-1,100 \mathrm{~kJ} \mathrm{~mol}^{-1}$ proton affinity range. For example, proton affinities of unsubstituted heteroaromatic molecules lie between $800 \mathrm{~kJ} \mathrm{~mol}^{-1}$ (e.g., furan, $\mathrm{PA}=812 \mathrm{~kJ} \mathrm{~mol}^{-1}$ ) and $900 \mathrm{~kJ} \mathrm{~mol}^{-1}$ (e.g., thiazole, $\mathrm{PA}=904 \mathrm{~kJ} \mathrm{~mol}^{-1}$ ). Similarly, monosubstituted benzenes have proton affinities situated between 750 and $950 \mathrm{~kJ} \mathrm{~mol}^{-1}$ (e.g., $\mathrm{PA}\left(\mathrm{C}_{6} \mathrm{H}_{5} \mathrm{~F}\right)=756 \mathrm{~kJ} \mathrm{~mol}^{-1}$ and $\mathrm{PA}\left(\mathrm{C}_{6} \mathrm{H}_{5} \mathrm{~N}\left(\mathrm{CH}_{3}\right)_{2}\right)=941 \mathrm{~kJ} \mathrm{~mol}^{-1}$. A careful examination of the details of the protonation of polyfunctional molecules reveals in fact very different and contrasting situations even in a same family of compounds. To take one example in the aromatic series, protonation of fluorobenzene occurs preferentially on the ring (Dopfer et al., 2005) while for aniline both the substituent and the cycle in ortho and para positions are likely candidates (Russo et al., 2000). Comparison of the protonation thermochemistry of some bifunctional aliphatic molecules such as diamines, diols, aminoalcohols with their monofunctional homologues also reveals surprising behavior. For example, the proton affinity of a primary amine is near $920 \mathrm{~kJ} \mathrm{~mol}^{-1}$ while from examination of Table 5 it appears that the proton affinities of diamines (1,2-ethanediamine, 1,3-propane diamine and 1,4butanediamine) are higher by ca. 30,70 , and $85 \mathrm{~kJ} \mathrm{~mol}^{-1}$ with respect to this value! This spectacular increase of basicity is also 
TABLE 4. Gas-phase protonation thermochemistry of primary standard bases ${ }^{j}$

\begin{tabular}{|c|c|c|c|c|c|}
\hline B & $\Delta_{\mathrm{f}} \mathrm{H}^{\circ}\left(\mathrm{BH}^{+}\right)^{\mathrm{a}}$ & $\Delta_{\mathrm{f}} \mathrm{H}^{\circ}(\mathrm{B})^{\mathrm{a,c}, \mathrm{c}}$ & PA(B $)^{2, d}$ & $\Delta \mathrm{S}_{\mathrm{p}}{ }^{\circ}(\mathrm{B})^{\mathrm{b}}$ & $\mathbf{G B}(\mathbf{B})^{a, e}$ \\
\hline Ketene & $657.0 \pm 1.5$ & $-47.7 \pm 2.5$ & $\underline{825.3} \pm 2.9$ & $2.4^{f}$ & $793.6 \pm 2.9$ \\
\hline \multirow[t]{2}{*}{ Isobutene } & $711.2 \pm 1.1$ & $-16.9 \pm 0.9$ & $\underline{802,1} \pm 1,4$ & $16.2^{\mathrm{f}}$ & $774.5 \pm 1.4$ \\
\hline & & & & $\underline{20}$ & $\underline{775.6} \pm 1.4$ \\
\hline Acetaldehyde & $595.4 \pm 0.4$ & $-166.1 \pm 0.5$ & $\underline{768.5} \pm 1.6$ & $1.3^{\mathrm{f}}, \underline{1.5}$ & $\underline{736.5} \pm 1.6$ \\
\hline \multirow[t]{2}{*}{ Propene } & $798.5 \pm 3.0$ & $20.1 \pm 0.8$ & $\underline{751.6} \pm 3.0$ & $10.2^{\mathrm{f}}$ & $722.2 \pm 3.0$ \\
\hline & & & & $\underline{12}$ & $\underline{722.7} \pm 3.0$ \\
\hline Formaldehyde & $708.5 \pm 0.8$ & $-108.8 \pm 0.8$ & $\underline{712.9} \pm 1.1$ & $9.5^{\mathrm{f}}$ & $\underline{683.3} \pm 1.1$ \\
\hline $\mathrm{H}_{2} \mathrm{~S}$ & $804.4 \pm 5.2$ & $-20.6 \pm 0.8$ & $\underline{705.0} \pm 5.3$ & $3.4^{\mathrm{f}},{\underline{4.3^{8}}}^{\mathrm{s}}$ & $\underline{673.8} \pm 5.3$ \\
\hline \multirow[t]{2}{*}{$\mathrm{H}_{2} \mathrm{O}$} & $592.6 \pm 5.0$ & $-241.8 \pm 0.04$ & $695.6 \pm 3.0$ & $3.9^{g}$ & $664.3 \pm 3.0$ \\
\hline & & & $\underline{691.0} \pm 3.0$ & $\underline{5}^{\mathrm{j}}, 4.8^{\mathrm{f}}$ & $\underline{660.0} \pm 3.0$ \\
\hline Ethylene & $901.8 \pm 1.5$ & $52.3 \pm 0.8$ & $\underline{680.5} \pm 1.7$ & $12.2^{\mathrm{f}},{\underline{11.5^{\mathrm{b}}}}^{\mathrm{b}}$ & $\underline{651.5} \pm 1.7$ \\
\hline $\mathrm{CO}$ & $825.6 \pm 2.7$ & $-110.5 \pm 0.2$ & $\underline{594.0} \pm 3.0$ & $3.8^{\mathrm{f}}, \underline{4.2^{\mathrm{i}}}$ & $\underline{562.8} \pm 2.8$ \\
\hline $\mathrm{CO}_{2}$ & $596.0 \pm 2.0$ & $-395.5 \pm 0.1$ & $\underline{540.5} \pm 2.0$ & $\underline{26.4^{\mathrm{f}}}$ & $\underline{515.8} \pm 2.0$ \\
\hline $\mathrm{NH}_{3}$ & 630.5 & $-45.9 \pm 0.4$ & $\underline{853.6}$ & $\underline{-6.4^{\mathrm{f}}},-6.6^{\mathrm{g}}$ & $\underline{819.0}$ \\
\hline \multicolumn{6}{|l|}{$\begin{array}{r}\text { electron between } \\
{ }^{\mathrm{d}} \mathrm{PA}(\mathrm{B})=\Delta_{\mathrm{f}} \\
{ }^{\mathrm{e}} \mathrm{GB}(\mathrm{B})=\mathrm{PA} \\
{ }^{\mathrm{f}} \mathrm{Theoretical} \\
{ }^{\mathrm{g}} \text { Estimated b } \\
{ }^{\mathrm{h}} \text { Mean value } \\
{ }^{\mathrm{i}} \text { From experi }\end{array}$} \\
\hline
\end{tabular}

observed with other diamino "superbases" or "proton sponges" (Gal, Maria, \& Raczynska, 2001) and finds its origin in interfunctional interactions.

\section{B. Characteristics Related to the Nature of the Basic Site}

The structural and energetic consequences of the protonation of species containing one or several basic sites, either a non-bonding electrons pair or a $\pi$-electrons system, has been extensively studied both experimentally and computationally during the years (Uggerud, 1992). It is out of the scope of the present review to completely detail this important domain. However, knowledge of the most significant structural changes induced by the protonation of currently encountered elementary basic sites may be useful to the understanding of the behavior of polyfunctional molecules. An overview of the structural changes induced by protonation on simple basic sites and the corresponding energetical consequences will be given here.

\section{Protonation of a Non-Bonding Electron Pair}

Protonation of a molecule at a non-bonding electron pair concerns species bearing elements of the groups 15-17 of the periodic system. The protonation process is accompanied by a considerable electron transfer from the lone pair donor, $\mathrm{X}$, to the incoming proton, which results in the formation of a covalent $\mathrm{X}-\mathrm{H}$ bond. This covalent bond formation is clearly attested by calculation of the electron density in the bonding region. Obviously, the electron density reorganization has also repercussions on the neighboring since the positive charge (or electron depletion) should be assumed by the totality of the molecular framework.

a. Hydrides. The role of the electronegativity of the protonated atom $\mathrm{X}$ on the proton affinity may be examined by considering the series of hydrides of groups $15-17$ of the periodic system together with the rare gas group 18. Table 6 gathers the relevant 


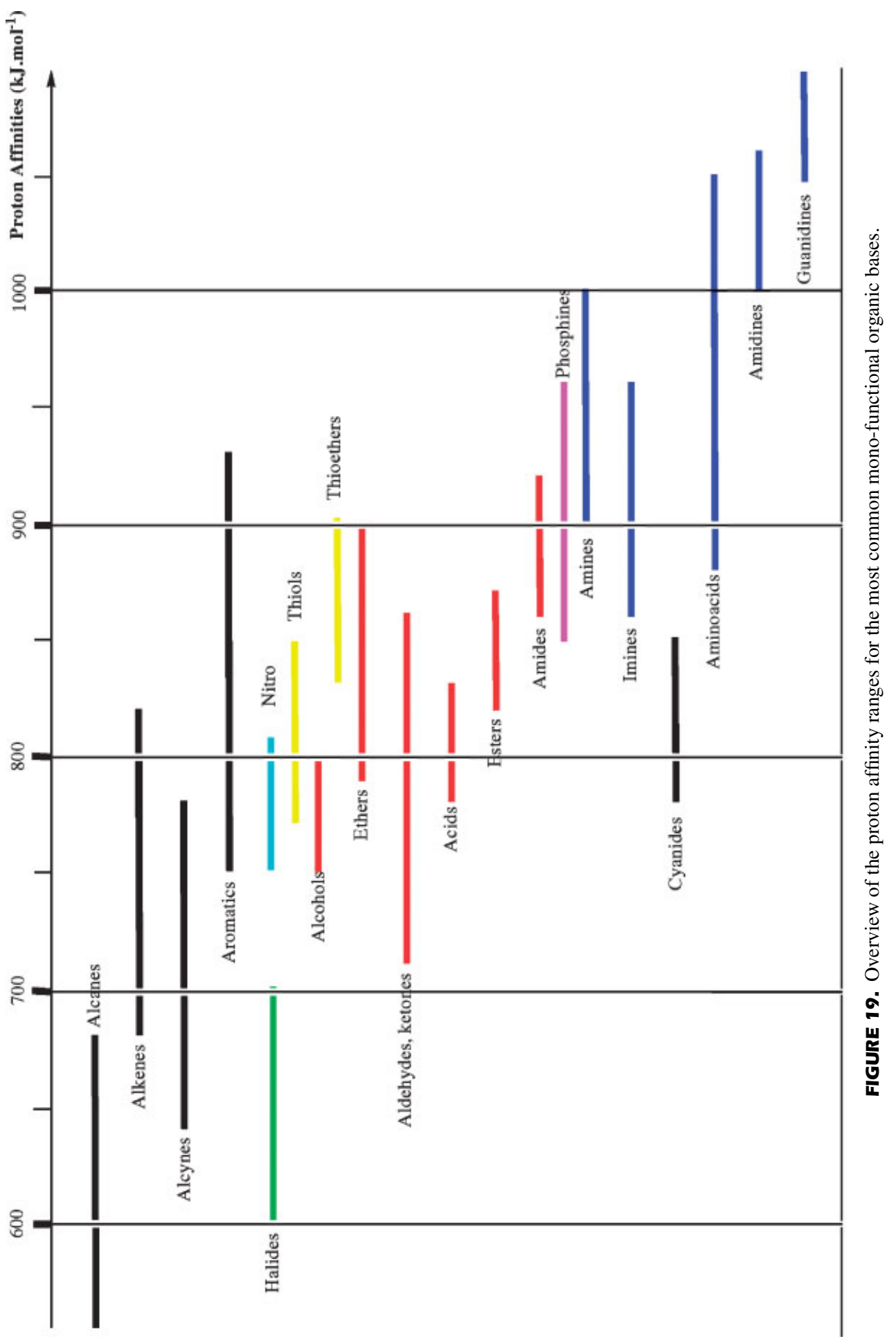


TABLE 5. Gas-phase basicities and proton affinities $\left(\mathrm{kJ} \mathrm{mol}^{-1}\right)^{\mathrm{a}}$ of selected organic molecules

\begin{tabular}{|c|c|c|}
\hline $\mathbf{M}$ & GB(M) & $\mathbf{P A}(\mathbf{M})$ \\
\hline methane & 520.6 & 543.5 \\
\hline ethane & 569.9 & 596.3 \\
\hline ethylene & 651.5 & 680.5 \\
\hline isobutene & 775.6 & 802.1 \\
\hline tetramethyl ethylene & 786 & 814 \\
\hline cyclohexane & 667 & 687 \\
\hline 1,3-butadiene & 757.6 & 783.4 \\
\hline acetylene & 616.7 & 641.4 \\
\hline allene & 745.8 & 775.3 \\
\hline propyne & 723.0 & 748.0 \\
\hline 2-butyne & 745.1 & 775.8 \\
\hline benzene & 725.4 & 750.4 \\
\hline 1,3,5-trimethyl benzene & 808.6 & 836.2 \\
\hline anthracene & 846.6 & 877.3 \\
\hline azulene & 928.0 & 958.7 \\
\hline methanol & 724.5 & 754.3 \\
\hline t-butanol & 772.2 & 802.6 \\
\hline dimethyl ether & 764.5 & 792.0 \\
\hline di-t-butyl ether & 860.0 & 887.4 \\
\hline ethylene oxide & 745.3 & 774.2 \\
\hline oxetane & 773.9 & 801.3 \\
\hline tetrahydrofurane & 794.7 & 822.1 \\
\hline tetrahydropyrane & 795.4 & 822.8 \\
\hline methanethiol & 742 & 773 \\
\hline i-propylthiol & 772 & 804 \\
\hline dimethylsulfide & 801 & 831 \\
\hline dimethyl-disulfide & 783 & 815 \\
\hline methyl-ethylsulfide & 815 & 846 \\
\hline ethylene sulfide & 778 & 807 \\
\hline trimethylene sulfide & 805 & 835 \\
\hline tetrahydrothiophene & 819 & 849 \\
\hline methylamine & 864.5 & 899 \\
\hline t-butylamine & 899.9 & 934.1 \\
\hline dimethylamine & 896.5 & 929.5 \\
\hline di-t-butylamine & 954.7 & 987.9 \\
\hline
\end{tabular}


TABLE 5. (Continued)

\begin{tabular}{|c|c|c|}
\hline trimethylamine & 918.1 & 948.9 \\
\hline tri-n-butylamine & 967.6 & 998.5 \\
\hline $\mathrm{N}$-methylpyrrolidine & 934.8 & 965.6 \\
\hline methylphosphine & 817.6 & 851.5 \\
\hline dimethylphosphine & 877.9 & 912.0 \\
\hline trimethylphosphine & 926.3 & 958.8 \\
\hline formaldehyde & 683.3 & 712.9 \\
\hline acetone & 782.1 & 812 \\
\hline 2,2,4,4-tetramethyl-3-pentanone & 831.5 & 861.3 \\
\hline cyclobutanone & 772.7 & 802.5 \\
\hline cyclopentanone & 794.0 & 823.7 \\
\hline cyclohexanone & 811.2 & 841.0 \\
\hline cycloheptanone & 815.9 & 845.6 \\
\hline acetic acid & 752.8 & 783.7 \\
\hline acetamide & 832.6 & 863.6 \\
\hline ethyl acetate & 804.7 & 835.7 \\
\hline dimethyl sulfoxide & 854 & 884 \\
\hline $\mathrm{CH}_{3} \mathrm{CSOCH}_{3}$ & 816 & 846 \\
\hline $\mathrm{CH}_{3} \mathrm{COSCH}_{3}$ & 798 & 829 \\
\hline formaldimine & 827 & 860 \\
\hline$\left(\mathrm{CH}_{3}\right)_{2}=\mathrm{NH}$ & 898.2 & 932.3 \\
\hline pyridine & 898.1 & 930.0 \\
\hline $\mathrm{HCN}$ & 681 & 712.9 \\
\hline $\mathrm{CH}_{3} \mathrm{CN}$ & 748 & 779.2 \\
\hline $\mathrm{CH}_{3} \mathrm{~F}$ & 571.5 & 598.9 \\
\hline $\mathrm{CH}_{3} \mathrm{Cl}$ & 621.1 & 647.3 \\
\hline $\mathrm{CH}_{3} \mathrm{Br}$ & 638.0 & 664.2 \\
\hline $\mathrm{CH}_{3} \mathrm{I}$ & 665.5 & 691.7 \\
\hline $\mathrm{CH}_{3} \mathrm{NO}_{2}$ & 721.6 & 754.6 \\
\hline $\mathrm{DBN}^{\mathrm{b}}$ & 1005.9 & 1038.3 \\
\hline $\mathrm{TBD}^{\mathrm{b}}$ & 1022.1 & 1054.6 \\
\hline 1,2-diaminoethane & 912.5 & 951.6 \\
\hline 1,3-diaminopropane & 940.0 & 987.0 \\
\hline 1,4-diaminobutane & 954.3 & 1005.6 \\
\hline 4-hydroxy-2-butanone & 832.6 & 878.5 \\
\hline
\end{tabular}


TABLE 5. (Continued)

\begin{tabular}{|c|c|c|}
\hline methoxyacetone $^{c}$ & 820 & 858 \\
\hline 4-methoxybutanone $e^{c}$ & 848.1 & 891.6 \\
\hline 5-methoxy-2-pentanone ${ }^{c}$ & 872 & 922.6 \\
\hline 2,5 -hexanedione ${ }^{d}$ & 852.7 & 893.2 \\
\hline 1,2-aminoethanol & 896.8 & 930.3 \\
\hline 1,3-aminopropanol & 917.3 & 962.5 \\
\hline 1,4-aminobutanol & 932.1 & 984.5 \\
\hline glycine $\left(\mathrm{NH}_{2} \mathrm{CH}_{2} \mathrm{COOH}\right)$ & 852.2 & 886.5 \\
\hline sarcosine $\left(\mathrm{NH}_{2} \mathrm{CH}_{2} \mathrm{CH}_{2} \mathrm{COOH}\right)$ & 888.7 & 921.2 \\
\hline
\end{tabular}

${ }^{\mathrm{a}}$ From Hunter and Lias (1998) unless otherwise indicated.

${ }^{\mathrm{b}} \mathrm{DBN}=1,5$-diazabicyclo[4,3,0]non-5-ene; TBD $=1,5,7$-triazabicyclo[4,4,0]dec-5-ene.

${ }^{\mathrm{c}}$ From Bouchoux et al. (2005).

${ }^{\mathrm{d}}$ From Akrour et al. (2007).

PA's values and the corresponding electronegativity coefficients, an illustration of the data is given in Figure 20.

It appears from Figure 20 that along the periods the proton affinity decreases as expected from the increase of electronegativity of the atoms (Table 6). The decrease in proton affinity is more pronounced for the elements of period 2 where the electronegativity rise is larger (1.5 units is the Mulliken scale from $\mathrm{NH}_{3}$ to $\mathrm{Ne}$ ). Note that the apparent exception of the two first elements of period 5 is perhaps not significant since the computed proton affinities may be too large for $\mathrm{TeH}_{2}$ and too low for $\mathrm{SbH}_{3}$ if we refer to the period 4 homologues (see the comparison between experimental and computed PA values for $\mathrm{AsH}_{3}$ and $\mathrm{SeH}_{2}$ in Table 6; Swart, Rösler, \& Bickelhaupt, 2006). The influence of electronegativity is also visible when looking at the evolution in a group. When descending the groups 16, 17, and 18, the proton affinity increases as expected from the decrease in electronegativity. However, the situation is opposite for the group 15 , the proton affinity decreases when passing from $\mathrm{NH}_{3}$ to $\mathrm{SbH}_{3}$. It should be observed that, if we except $\mathrm{N}$, the first member of this group, the electronegativity is close to, or even lower than, that of the hydrogen. It may be supposed that, for these limiting cases, the electronegativity is probably not the only property to take into account in the understanding of the behavior of these elements during the protonation.

b. Effects of the protonation on the $X$ bonding. Protonation of simple alkyl derivatives $\mathrm{RX}(\mathrm{X}=$ elements of groups 15,16 , and 17) reveals several structural and energetic features which were explored by computational chemistry (Alcami et al., 1990; Boyd et al., 1995; Boyd \& Boyd, 1997, 1999; Mayer et al., 1997). Alcami et al. (1990) investigate the bonding perturbation caused by the protonation on small bases by the atoms in molecule (AIM) theory (Bader, 1990). They observed that, when protonation takes place at an electronegative center $\mathrm{X}$, the $\mathrm{C}-\mathrm{X}$ bond becomes weakened. This bond weakening is reflected by a lower value of the electron density within the bonding region and by an increase of the $\mathrm{C}-\mathrm{X}$ bond length. Figure 21 shows the optimized geometries of neutral and protonated ethylamine, ethanol and ethyl fluoride which clearly illustrate this $\mathrm{C}-\mathrm{X}$ bond elongation in the protonated species.

The $\mathrm{C}-\mathrm{X}$ bond weakening induced by the protonation may be qualitatively explained by the tendency of $\mathrm{X}$, after the creation

TABLE 6. Proton affinities $\left(\mathrm{kJ} \mathrm{mol}^{-1}\right)^{\mathrm{a}}$ of hydrides and noble gases (electronegativity coefficients $\chi$ of the atoms)

\begin{tabular}{cccccccc}
\hline $\mathrm{NH}_{3}$ & 854 & $\mathrm{OH}_{2}$ & 691 & $\mathrm{FH}$ & 484 & $\mathrm{Ne}$ & 199 \\
& $3.04^{\mathrm{b}}$ & & 3.44 & & 3.98 & & - \\
& $3.08^{\circ}$ & & 3.22 & & 4.43 & & 4.6 \\
$\mathrm{PH}_{3}$ & 785 & $\mathrm{SH}_{2}$ & 705 & $\mathrm{ClH}$ & 557 & $\mathrm{Ar}$ & 369 \\
& 2.19 & & 2.58 & & 3.16 & & - \\
& 2.39 & & 2.65 & & 3.54 & & 3.36 \\
$\mathrm{AsH}_{3}$ & $748(739)$ & $\mathrm{SeH}_{2}$ & $708(721)$ & $\mathrm{BrH}$ & 584 & $\mathrm{Kr}$ & 425 \\
& 2.18 & & 2.55 & & 2.96 & & 3.00 \\
& 2.26 & & 2.51 & & 3.24 & & 2.98 \\
$\mathrm{SbH}_{3}$ & $(734)$ & $\mathrm{TeH}_{2}$ & $(746)$ & $\mathrm{IH}$ & 627 & $\mathrm{Xe}$ & 499 \\
& 2.05 & & 2.10 & & 2.66 & & 2.60 \\
& 2.06 & & 2.34 & & 2.88 & & 2.59 \\
\hline
\end{tabular}

${ }^{a}$ Experimental proton affinity values from Hunter and Lias (1998), into parentheses calculated values from Swart et al. (2006).

${ }^{\mathrm{b}}$ Pauling electronegativity, in this scale $\chi_{\mathrm{C}}=2.55$ and $\chi_{\mathrm{H}}=2.20$.

${ }^{\mathrm{c}}$ Mulliken electronegativity, in this scale $\chi_{\mathrm{C}}=2.67$ and $\chi_{\mathrm{H}}=3.06$. 


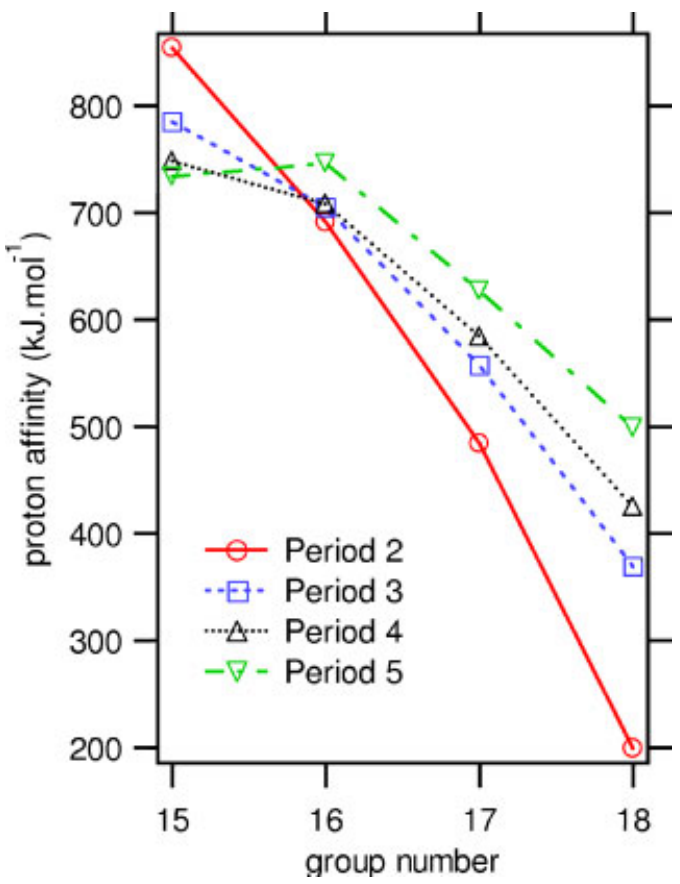

FIGURE 20. Evolution of the proton affinities of hydrides of atoms of groups 15, 16, and 17 and of noble gases (group 18) along the periods. [Color figure can be viewed in the online issue, which is available at www.interscience.wiley.com.]

of a covalent $\mathrm{X}-\mathrm{H}$ bond during the protonation process, to recover its electroneutrality by lowering the electron density along bonds with less electronegative atoms. The obvious consequence is that the $\mathrm{C}-\mathrm{X}$ bond becomes depopulated and therefore lengthened. Another equivalent mean to interpret this structural effect is to describe the $\mathrm{C}-\mathrm{X}$ bond by two limiting structures (Mayer et al., 1997), a single-bond covalent structure 1c, and an ionic structure $\mathbf{1 i}$ for the neutral, and, similarly $\mathbf{1 H c}$ and $\mathbf{1 H i}$ for the protonated form (Scheme 2).

In the neutral molecule, the bonded structure $1 \mathrm{c}$ dominates, but in its $\mathrm{X}$ protonated form the non-bonding structure $\mathbf{1 H i}$ becomes significant resulting in a longer $\mathrm{C}-\mathrm{X}$ bond. Following this reasoning, it is expected that the participation of $\mathbf{1 H i}$ in the description of the protonated molecule should be more important for electronegative atoms X. This is indeed confirmed by the calculations. As illustrated in Figure 21 for the elements of the second period the increase in $\mathrm{C}-\mathrm{X}$ bond length is equal to $4 \%$, $10 \%$, and $29 \%$ for $\mathrm{X}=\mathrm{N}, \mathrm{O}$, and F, respectively. Data presented in Table 7, obtained at a different theoretical level for a largest part of the periodic system shows a systematic bond elongation in each period when passing from group 15 to group 17 elements (Boyd et al., 1995; Boyd \& Boyd, 1997, 1999). It is evident that the increase in $\mathrm{C}-\mathrm{X}$ bond length when passing from $\mathrm{C}_{2} \mathrm{H}_{5} \mathrm{X}$ to its protonated form $\mathrm{C}_{2} \mathrm{H}_{5} \mathrm{XH}^{+}$is maximum for the halogen derivatives which results in a dramatic situation for the fluoride derivatives. This is clearly exemplified with ethyl fluoride in Figure 21 where the conservation of a C-FH covalent bond in protonated fluoride may be questioned. In fact, the corresponding species may be more properly viewed as complexes between a carbocation and a molecule of hydrogen fluorine. This situation is

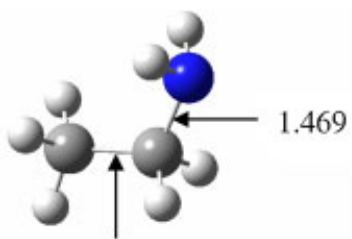

1.527
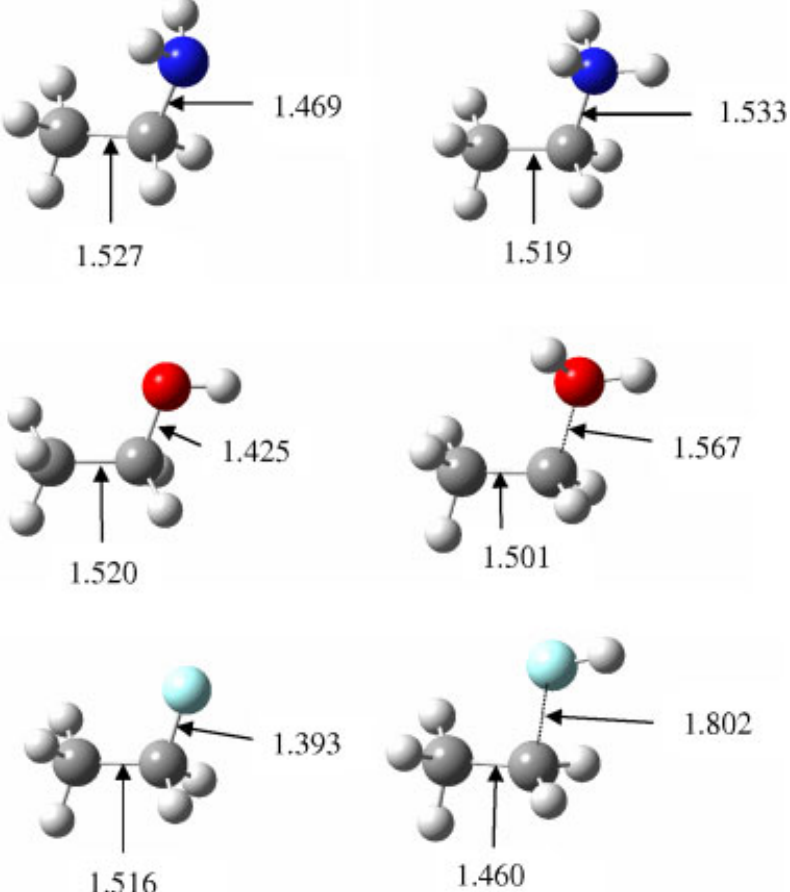

FIGURE 21. B3LYP/6-31G(d) optimized geometries of neutral and protonated ethylamine, ethanol and fluoroethane. [Color figure can be viewed in the online issue, which is available at www.interscience. wiley.com.]

even more accentuated when the cation is particularly stable, as for example tertiary carbocation (Abboud et al., 1994).

The weakening of a bond may be also estimated from the corresponding dissociation energy. A direct comparison between bond dissociation energies of neutral and protonated species is however not easy since, most of the time, homolytic dissociation is favored for the neutral but heterolytic dissociation is preferred in the protonated molecules. Nevertheless, a comparison between the two types of bond dissociation energies is instructive. Table 7 contains the relevant information for the above-mentioned $\mathrm{C}_{2} \mathrm{H}_{5} \mathrm{X} / \mathrm{C}_{2} \mathrm{H}_{5} \mathrm{XH}^{+}$system. It is a well-known result that homolytic $\mathrm{C}-\mathrm{X}$ bond dissociation energies increases with the electronegativity difference between $X$ and $C$ (Coote et al., 2003). Thus the behavior of $\mathrm{C}_{2} \mathrm{H}_{5} \mathrm{X}$ molecules is conform to the expectations. By contrast, what is evident from Table 7 is that the heterolytic $\mathrm{C}-\mathrm{X}$ bond dissociation energies of $\mathrm{C}_{2} \mathrm{H}_{5} \mathrm{XH}^{+}$decrease dramatically with increasing the electronegativity difference. This is

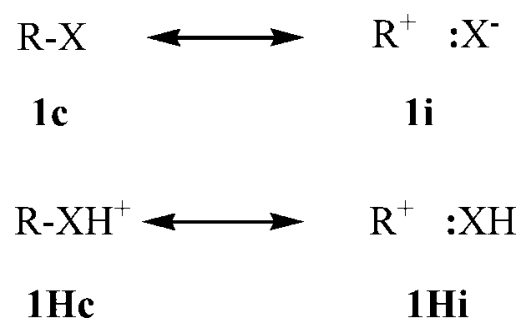

SCHEME 2. 
TABLE 7. Effect of the $\mathrm{X}$ protonation of $\mathrm{CH}_{3} \mathrm{CH}_{2} \mathrm{X}$ molecules on the $\mathrm{C}-\mathrm{X}$ bond length $\left(d_{\mathrm{C}-\mathrm{X}}\right)$ and dissociation enthalpy

\begin{tabular}{lllllll}
\hline $\mathrm{X}$ & $\begin{array}{l}\mathrm{d}_{\mathrm{C}-\mathrm{X}}(\AA)^{\mathrm{a}} \\
\text { neutral }\end{array}$ & $\begin{array}{l}\mathrm{d}_{\mathrm{C}-\mathrm{xH}}(\AA)^{\mathrm{a}} \\
\text { protonated }\end{array}$ & $\Delta \mathrm{d}_{\mathrm{CX}}(\AA)$ & $\Delta \chi_{\mathrm{X}}^{\mathrm{b}}$ & $\begin{array}{l}\Delta_{\mathrm{CXH}} \mathrm{H}^{\circ}(\mathrm{kJ} / \mathrm{mol})^{\mathrm{c}} \\
\text { heterolytic }\end{array}$ & $\begin{array}{l}\Delta_{\mathrm{CX}} \mathrm{H}^{\circ}(\mathrm{kJ} / \mathrm{mol})^{\mathrm{c}} \\
\text { homolytic }\end{array}$ \\
\hline $\mathrm{NH}_{2}$ & 1.462 & 1.506 & 0.044 & 0.49 & $278(302)$ & $357(365)$ \\
$\mathrm{OH}$ & 1.426 & 1.513 & 0.087 & 0.89 & $165(147)$ & $392(400)$ \\
$\mathrm{F}$ & 1.403 & 1.627 & 0.224 & 1.43 & $16(36)$ & $460(479)$ \\
& & & & & & \\
$\mathrm{PH}_{2}$ & 1.858 & 1.807 & -0.051 & -0.36 & $(293)$ & $(286)$ \\
$\mathrm{SH}$ & 1.823 & 1.835 & 0.012 & 0.03 & $187(198)$ & $303(301)$ \\
$\mathrm{Cl}$ & 1.788 & 1.906 & 0.118 & 0.61 & $141(64)$ & $351(341)$ \\
& & & & & & \\
$\mathrm{AsH}$ & 1.958 & 1.899 & -0.059 & -0.37 & $(294)$ & $(290)$ \\
$\mathrm{SeH}$ & 1.947 & 1.964 & 0.017 & 0.00 & $(231)$ & $(301)$ \\
$\mathrm{Br}$ & 1.958 & 2.057 & 0.089 & 0.41 & $82(-11)$ & $292(317)$ \\
\hline
\end{tabular}

${ }^{\mathrm{a}} d_{\mathrm{C}-\mathrm{X}}$ and $d_{\mathrm{C}-\mathrm{XH}}$ are the $\mathrm{C}-\mathrm{X}$ bond lengths in the neutral and protonated species, respectively. MP2/6-31G(d) calculations from (Boyd et al., 1995; Boyd \& Boyd, 1995, 1997).

${ }^{\mathrm{b}}$ Pauling electronegativity difference between $\mathrm{X}$ and $\mathrm{C}$.

${ }^{\mathrm{c}}$ Bond dissociation energies, heterolytic for the protonated molecules, homolytic for the neutral. Experimental values obtained using heats of formation and proton affinities from Lias and Bartmess (2000) except the proton affinities oh ethyl halides from (Bouchoux et al., 2001a). Into parentheses calculated values from (Boyd \& Boyd, 1997, 1999).

clearly in line with the corresponding bond elongation described above and with a significant participation of structure 1 Hi in the description of these protonated molecules (Mayer et al., 1997). Finally, it may be noted that the $\mathrm{C}-\mathrm{X}$ bond elongation is generally accompanied by a slight shortening of the adjacent $\mathrm{CH}$ or $\mathrm{CC}$ bonds. This is for example apparent in Figure 21 for the ethyl derivatives. This bond reinforcement, which closely parallels the $\mathrm{C}-\mathrm{X}$ bond weakening, is a consequence of the polarization and hyperconjugative effects inside the ethyl moiety in $\mathrm{C}_{2} \mathrm{H}_{5} \mathrm{XH}^{+}$ ions (Mayer et al., 1997).

At the opposite, direct $\mathrm{C}-\mathrm{X}$ bond shortening is also a possible result of the protonation. This is for example apparent in Table 7 for $\mathrm{X}=\mathrm{PH}_{2}$ or $\mathrm{AsH}_{2}$ where the $\mathrm{C}-\mathrm{X}$ bond is shortened by $0.05 \AA$ in $\mathrm{C}_{2} \mathrm{H}_{5} \mathrm{XH}^{+}$with respect to $\mathrm{C}_{2} \mathrm{H}_{5} \mathrm{X}$. Also apparent is the fact that the electronegativity of $\mathrm{X}$ is that time less than that of the carbon. This has been rationalized in the following way (Alcami et al., 1990), if protonation occur on an atom X less electronegative than its surrounding, there is no trend for it to recover its neutrality and the protonation has in fact the only consequence to polarize the $\mathrm{C}-\mathrm{X}$ bond and thus to reinforce it. In other words, structure 1Hc becomes prominent and the corresponding covalent bond is reinforced by the polarization created by the charge.

Examples of bases, in which basic sites are in vicinal position, were presented by Alcami et al. (1990), an interesting case is hydroxylamine which may protonate either on the nitrogen or on the oxygen atom. In line with the preceding discussion, the $\mathrm{N}-\mathrm{O}$ bond is elongated (by $0.047 \AA$ ) when protonation occurs on the oxygen atom, and shortened (by $0.039 \AA$ ) when protonation occurs on the less electronegative nitrogen atom.

c. Carbonyl group. Protonation of aldehydes, ketones, acids, esters and amides preferentially occurs on the carbonyl oxygen. During this process, the $\mathrm{C}=\mathrm{O}$ bond length is slightly elongated, as expected from the electron depopulation in this bonding region due to the formation of the $\mathrm{O}-\mathrm{H}$ bond. Several examples are presented in Figure 22 (B3LYP/6-31G(d) calculation).

For example, the $\mathrm{C}=\mathrm{O}$ bond of acetone passes from $1.216 \AA$ in the molecule to $1.281 \AA$ in the $\left(\mathrm{CH}_{3}\right)_{2} \mathrm{COH}^{+}$ion (Fig. 22a). Interestingly enough, the vicinal bonds are shortened during the protonation of the carbonyl group. Considering again the acetone molecule, the $\mathrm{CH}_{3}-\mathrm{CO}$ bond passes from $1.521 \AA$ in the neutral to 1.474 and $1.478 \AA$ in the ion. This CC bond reinforcement is partly due to the hyperconjugative electron donation of the alkyl groups to the $\pi^{*}$ orbital of the $\mathrm{COH}^{+}$moiety, and partly to the polarization of the $\mathrm{C}-\mathrm{C}$ bond resulting from the positive charge located on the carbon of the $\mathrm{COH}^{+}$moiety (Mayer et al., 1997).

These changes in bond lengths are also accompanied by a change in bond angle at the carbonyl carbon. The effect of the CCC angle variation between $80^{\circ}$ and $140^{\circ}$ upon the total energies of the neutral and the protonated acetone is illustrated by Figure 23. As expected, the energy of both species pass trough a minimum close to $\mathrm{CCC}=120^{\circ}$. However, the precise bond angle value is lower than $120^{\circ}$ for the neutral and larger than $120^{\circ}$ for the protonated form. As shown in Figure 22a, the CCC angle in acetone is equal to $116.5^{\circ}$ but is increased to $122.9^{\circ}$ in protonated acetone. This angle opening may be explained by a larger electrostatic repulsion between the two methyl groups in the protonated form with respect to its neutral counterpart. Another remark emerging from examination of Figure 23 is that both neutral and protonated species exhibit similar amplitude in the variation of their total energies with the CCC angle. This simple shift of the curves along the $\mathrm{CCC}$ angle axis gives rise to a quasi-linear relationship between the "vertical" proton affinity of acetone with the CCC angle as illustrated by the upper part of Figure 23

The increase in proton affinity of the carbonyl group with the $\mathrm{CCC}$ angle is experimentally verified for the cyclanone series. As indicated in Table 5, the proton affinity of cyclobutanone is equal to $802 \mathrm{~kJ} \mathrm{~mol}^{-1}$ and progressively increases with the size of the cycle to attain $845 \mathrm{~kJ} \mathrm{~mol}^{-1}$ with cycloheptanone (Bouchoux \& 

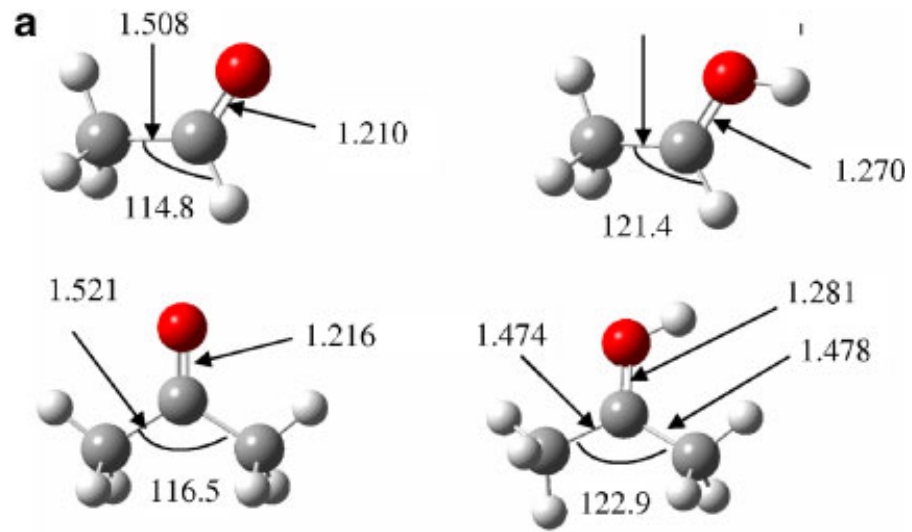

b

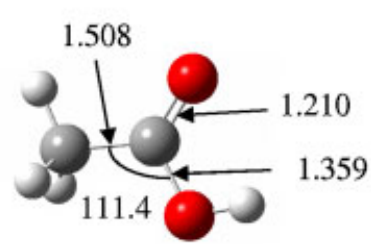

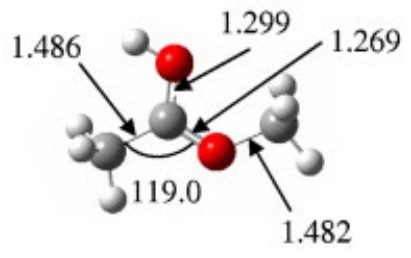

as

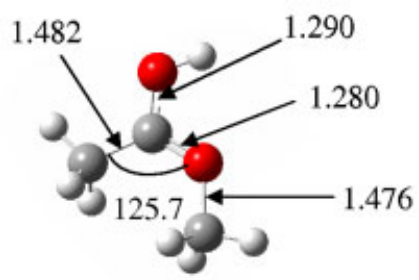

sa

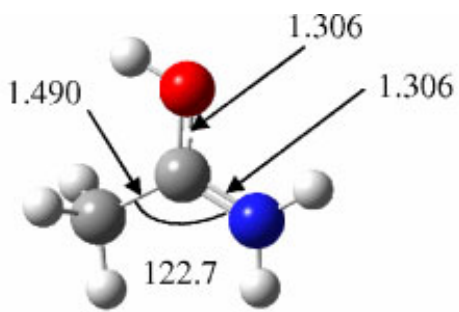

$a$
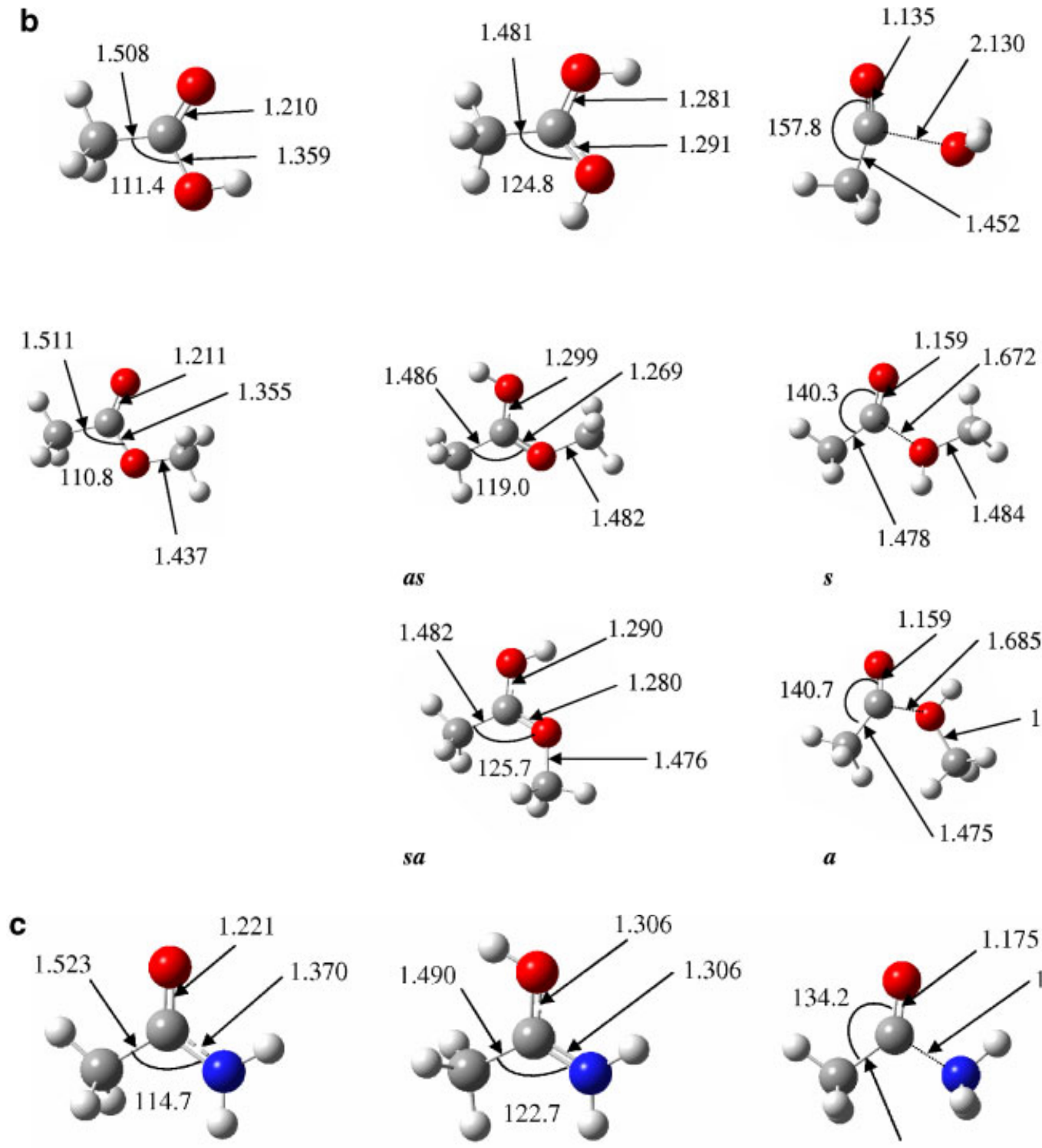

$\boldsymbol{s}$

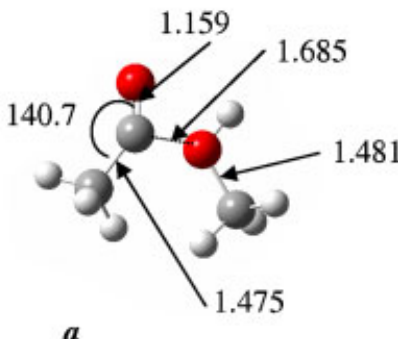

$a$

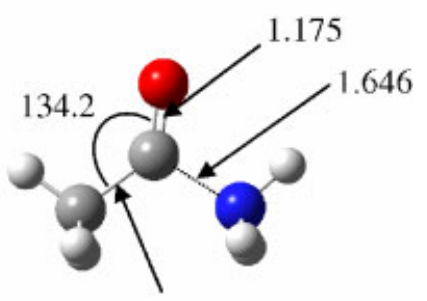

1.487

FIGURE 22. B3LYP/6-31G(d) optimized geometries of neutral and protonated (a) acetaldehyde and acetone, (b) acetic acid and methyl acetate, and (c) acetamide. [Color figure can be viewed in the online issue, which is available at www.interscience.wiley.com.] 

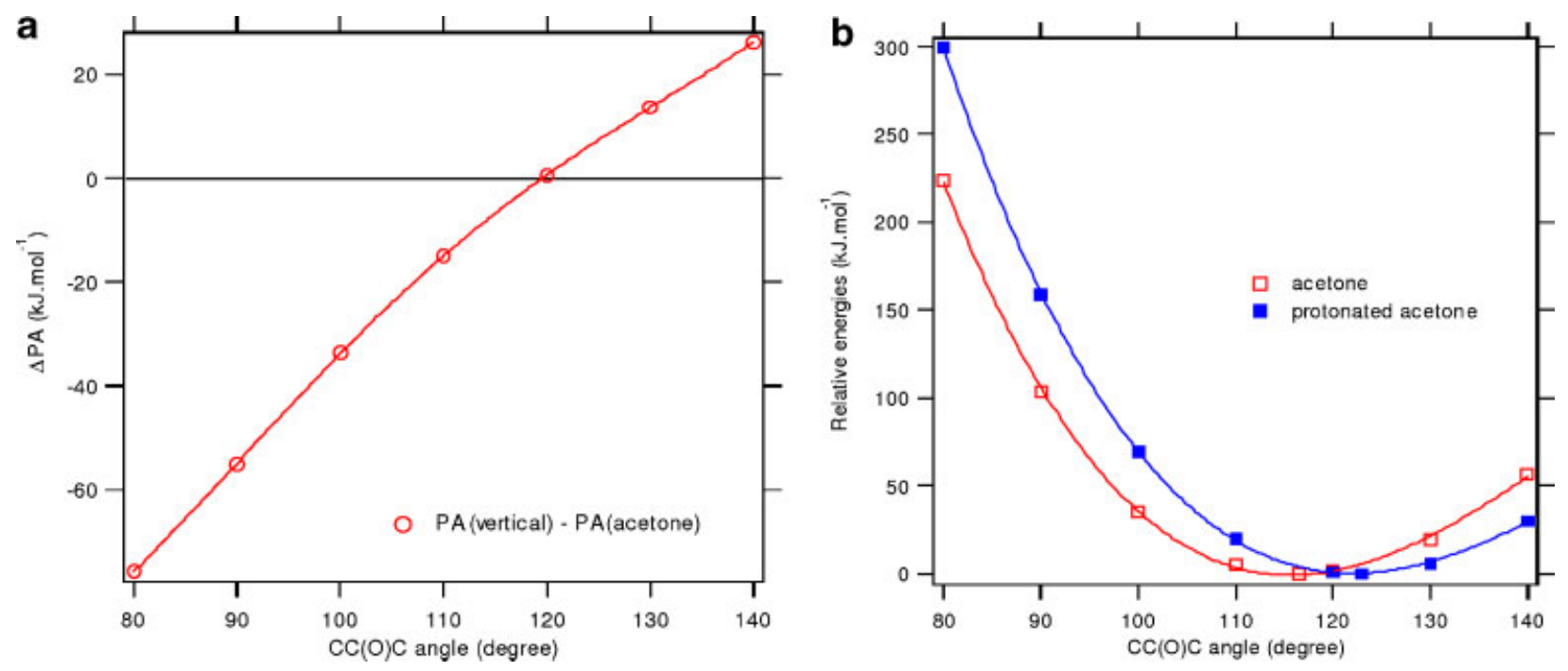

FIGURE 23. Variations of the proton affinity of acetone and the corresponding relative energies of acetone and protonated acetone as a function of the CCC bond angle (B3LYP/6-31G(d) calculations). [Color figure can be viewed in the online issue, which is available at www.interscience.wiley.com.]

Houriet, 1984). Similar observations were made for lactones (Bordeje et al., 1993; Bouchoux et al., 1995) and lactams (Abboud et al., 1992).

Protonation of carboxylic acids on the carbonyl oxygen may give rise to three conformers which differ by the syn or antiHOCO arrangements. The most stable form corresponds to a syn-anti conformation (Fig. 22b). In the case of protonated acetic acid, the two other conformers anti-anti and syn-syn are less stable by 15 and $19 \mathrm{~kJ} \mathrm{~mol}^{-1}$, respectively, at the B3LYP/6$31 \mathrm{G}(\mathrm{d})$ level. It is noteworthy that protonation on the hydroxyl oxygen of carboxylic acids leads to a highly energetic species characterized by a considerable $\mathrm{C}-\mathrm{OH}_{2}$ bond elongation. As shown in Figure $22 \mathrm{~b}$ the $\mathrm{CO}$ distance is equal to $2.13 \AA$ in the hydroxyl protonated acetic acid and, in fact, the final structure may be better considered as a complex between an acylium ion (note the $\mathrm{CCO}$ bond angle of $157.8^{\circ}$ which tends toward a linear arrangement of the heavy atoms and the $\mathrm{CO}$ bond shortening) and a water molecule. The relative energy of this structure is $77 \mathrm{~kJ} \mathrm{~mol}^{-1}$ above the syn-anti carbonyl protonated form in the case of acetic acid (B3LYP/6-31G(d) level calculations).

Esters show behavior comparable to that presented above for carboxylic acids. The case of methyl acetate will illustrate this statement. If the neutral molecule is clearly more stable in its syn OCOC conformation (see Fig. 22b), upon protonation, the two structures anti-HOCO-syn-OCOC and syn-HOCO-anti-OCOC (as and sa, Fig. 22b) are of comparable stabilities (the former is $3 \mathrm{~kJ} \mathrm{~mol}^{-1}$ below the latter at the B3LYP/6-31G(d) level). These two protonated structures are characterized by a significant shortening of the $\mathrm{C}(\mathrm{O})-\mathrm{OCH}_{3}$ bond. By contrast, when protonation occurs on the ether like oxygen, this $\mathrm{C}-\mathrm{O}$ bond is dramatically elongated (structures $s$ and $a$, Fig. 22b). It is observed however that the $\mathrm{C}-\mathrm{O}$ bond elongation is less pronounced for ester than for the carboxylic acid itself. This may be explained by the fact that the alkyl group of the ether moiety share a part of the electron depletion thus leading to a less dramatic depopulation of the $\mathrm{C}(\mathrm{O})-\mathrm{O}$ bond. As a corollary, the acyl moiety is less comparable in structure to an acylium ion (the CCO angle is close to $140^{\circ}$, not $180^{\circ}$ for example). The energies of structure $s$ and $a$, relative to $a s$, are 88 and $92 \mathrm{~kJ} \mathrm{~mol}^{-1}$ respectively.

Protonation of acetamide at the carbonyl may give rise to two conformers, the anti-HOCN structure $a$ presented in Figure $22 \mathrm{c}$ is more stable than its syn homologue by $61 \mathrm{~kJ} \mathrm{~mol}^{-1}$. The nitrogen protonated structure $n$, characterized, as expected, by an elongated C-N bond, lies $61 \mathrm{~kJ} \mathrm{~mol}^{-1}$ above $a$ (Fig. 22c).

d. Imines, cyanides, nitro. Protonation of imino nitrogen presents the same characteristics as that observed for the carbonyl group. As exemplified with acetaldimine $\mathrm{CH}_{3} \mathrm{CHNH}$ in Figure 24, protonation is accompanied by a lengthening of the $\mathrm{C}=\mathrm{N}$ bond and a shortening of the $\mathrm{C}-\mathrm{C}$ bond, simultaneously, the $\mathrm{HC}(\mathrm{N}) \mathrm{C}$ angle is increased. This is comparable to the changes observed for acetaldehyde, though to a rather modest level.

Cyanides offer a specific behavior since protonation on the nitrogen is accompanied by a shortening of the $\mathrm{CN}$ bond (Fig. 24), a result at variance with that observed with imine or carbonyl bases. More classical is the shortening of the $\mathrm{CC}$ bond, illustrating again the hyperconjugative and polarizability effects of the methyl group (Mayer et al., 1997).

During protonation of nitromethane, a modest shortening of the $\mathrm{CN}$ bond is noted (Fig. 24; Mayer et al., 1997). The most spectacular changes are located on the ONO moiety where one NO bond is elongated by $0.1 \AA$ upon protonation while the vicinal NO bond is shortened by $\sim 0.05 \AA$.

e. Hybridization effect. Since $s$ atomic orbitals are much lower in energy than the $p$ atomic orbitals of a given atom, the electronegativity of hybridized orbital increases with their $s$ character. For example, in the Pauling scale, the electronegativity of the carbon hybrid orbital is $2.48,2.75$, and 3.29 for $s p^{3}$ to $s p^{2}$ 

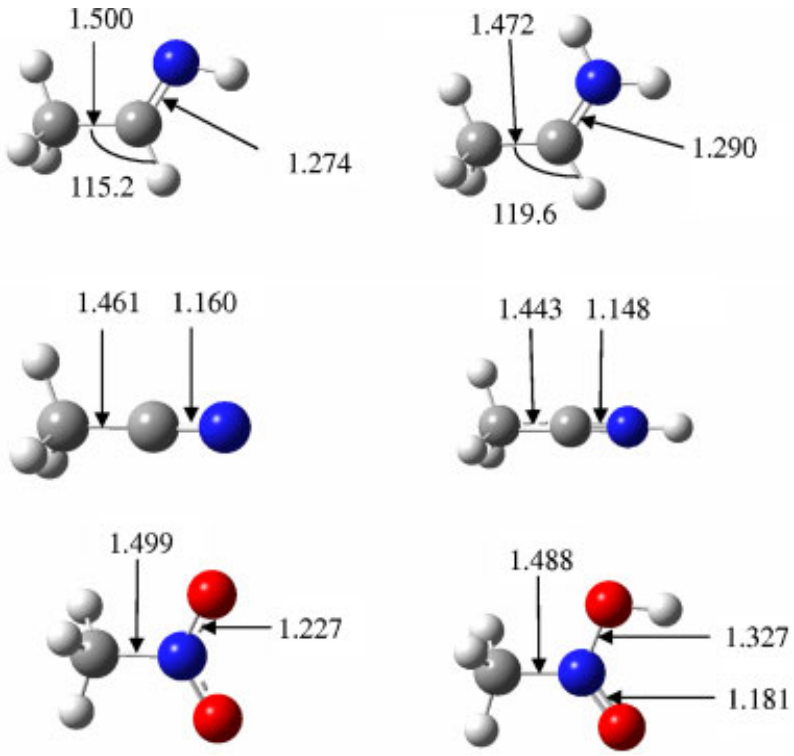

FIGURE 24. B $3 L Y P / 6-31 \mathrm{G}(\mathrm{d})$ optimized geometries of neutral and protonated acetaldimine, methyl cyanide and nitromethane. [Color figure can be viewed in the online issue, which is available at www. interscience.wiley.com.]

and $s p$ hybridization. When the lone pair responsible of the basicity is located in a hybrid orbital which presents a large $s$ character, this lone pair is more tightly bound and therefore the basicity of the molecule decreases. It is consequently expected that the proton affinity of molecules containing a basic atom $\mathrm{X}$ will decrease when passing from $s p^{3}$ to $s p^{2}$ and $s p$ hybridization state of $\mathrm{X}$.

It is indeed observed that the proton affinities of methylamine $\left(899 \mathrm{~kJ} \mathrm{~mol}^{-1}\right)$, methyleneimine $\left(863 \mathrm{~kJ} \mathrm{~mol}^{-1}\right)$, and hydrogen cyanide $\left(713 \mathrm{~kJ} \mathrm{~mol}^{-1}\right)$ decreases in that order. Similarly the proton affinity of formaldehyde $\left(713 \mathrm{~kJ} \mathrm{~mol}^{-1}\right)$ is lower than that of methanol $\left(754 \mathrm{~kJ} \mathrm{~mol}^{-1}\right)$. This reasoning should be however completed by the observation that resonance stabilization occurs for the protonated species resulting from $s p$ and $s p^{2}$ hybridization, not for $s p^{3}$ (Scheme 3).

Resonance offers an extra stabilization of the protonated forms which tends to increases the proton affinity and thus acts in the opposite direction of the hybridization-electronegativity effect. The observed decreases in proton affinities are consequently underestimates of the latter effect.
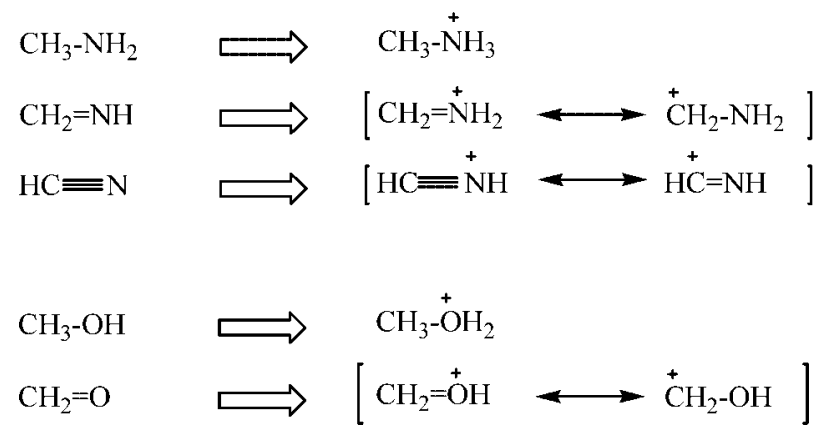

SCHEME 3.

\section{Protonation on $\pi$-Electron Systems}

Protonation of an alkene with an unsymetrical $\pi$-bond is expected to produce two different alkyl ions (Scheme 4).

Quantum chemistry computation and experiment show however that hydride ion migrations are facile processes on alkyl cations thus leading rapidly to a mixture of structures when alcenes (or polyenes) are protonated. Moreover there are convincing evidences that primary carbocations are not stable species and rearrange spontaneously by 1,2-hydride (or alkyl and aryl) ion shift to secondary or tertiary cations (Koch \& Liu, 1989; Sieber et al., 1993; Aubry \& Holmes, 1998).

A similar situation is expected for acetylenic compounds, protonation at both sides of the triple bond may give rise to two alkenyl ions (Scheme 5). Alkenyl cations may rearrange readily to more stable allylic cations although a non-negligible activation barrier seems to separate efficiently the two kinds of isomers (van der Rest et al., 1997).

The case of protonated ethylene is peculiar since, to avoid the formation of a primary carbocation, only a partial 1,2-hydride ion shift is possible which lead to the bridged structure $\boldsymbol{a}$ (Scheme 6). Note that the classical structure $\boldsymbol{b}$ is a transition structure interconnecting two structures $\boldsymbol{a}$ for the scrambling of the proton and is situated $27 \mathrm{~kJ} \mathrm{~mol}^{-1}$ above $\boldsymbol{a}$ (Ruscic, Berkowitz, \& Pople, 1989). Protonation of acetylene is also particular in that sense that the bridged structure $\boldsymbol{a}^{\prime}$ (Scheme 6) is more stable than the classical vinyl cation $\boldsymbol{b}^{\prime}$ by ca. $18 \mathrm{~kJ} \mathrm{~mol}^{-1}$ (G2 calculation at 298 K, Glukhovtsev \& Bach, 1998) and that there is little or no barrier separating the bridged and open structures (Lindh, Roos, \& Kraemer, 1987; Curtiss \& Pople, 1988).

a. $\pi$-Electron resonance. It is well known that a carbocation is stabilized if the positive charge is delocalized by conjugation with a $\pi$-electron system. Protonation of polyenes is consequently expected to give increasingly stabilized $\mathrm{MH}^{+}$forms when the number of conjugated double bond increases. This leads to a parallel increase in proton affinity as attested for example by the PA values of propene $\left(751.6 \mathrm{~kJ} \mathrm{~mol}^{-1}\right.$, Table 4), 1,3-butadiene (783.4 $\mathrm{kJ} \mathrm{mol}^{-1}$, Table 5) and 1,3,5-cycloheptatriene (833.0 $\mathrm{kJ} \mathrm{mol}^{-1}$, Salpin et al., 2003).

There is no doubt that benzene is preferentially protonated at the ring to give benzenium ion $\boldsymbol{a}^{\prime \prime}$ (Scheme 7; Kuck, 1990a, 2005). Detailed quantum chemical computation shows that the face protonated $\pi$-complex structure $\boldsymbol{b}^{\prime \prime}$ is not a minimum in the potential energy surface and that it corresponds to an energy level situated $\sim 200 \mathrm{~kJ} \mathrm{~mol}^{-1}$ above $\boldsymbol{a}^{\prime \prime}$ (Glukhovtsev et al., 1995). The non-existence of $\boldsymbol{b}^{\prime \prime}$ as a stable specie in the gas-phase is also supported by experimental studies (Schröeder et al., 2004). The proton affinity of benzene $\left(750 \mathrm{~kJ} \mathrm{~mol}^{-1}\right.$, Table 5$)$ is close to that of propene $\left(751.6 \mathrm{~kJ} \mathrm{~mol}^{-1}\right.$, Table 4$)$, that is, significantly lower

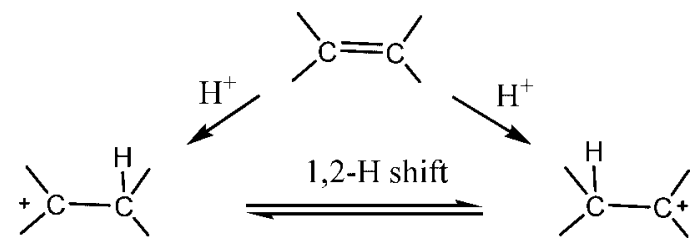

SCHEME 4. 


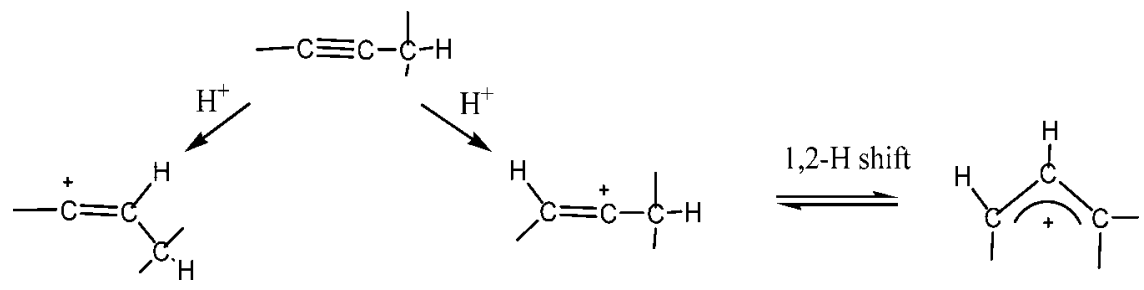

SCHEME 5.

than that of conjugated polyenes containing the same number of $\pi$-electrons. This is a clear demonstration that the neutral molecule benefits of a strong stabilization, obviously due to the aromaticity of the $\pi$-electrons system, thus leading to a decrease in proton affinity.

Isomerization of benzenium ions $\boldsymbol{a}^{\prime \prime}$ involve 1,2-hydride ion migration leading to a complete exchange of hydrogen positions in the ring. The transition structure for this "hydrogen ring walking", $\boldsymbol{c}^{\prime \prime}$ (Scheme 7) is a edge protonated structure situated $\sim 35 \mathrm{~kJ} \mathrm{~mol}^{-1}$ above $\boldsymbol{a}^{\prime \prime}$ (Glukhovtsev et al., 1995). Other possibilities of isomerization of the benzenium ion $\boldsymbol{a}^{\prime \prime}$ involving ring contraction into the various forms of protonated fulvene have been also explored by molecular ab initio calculations (Bouchoux, Yanez, \& Mo, 1999).

Alkyl substituted benzenes (Kuck, 1990a,b, 2005), electrondonor substituted benzenes or polynuclear aromatic hydrocarbons (Aue, Guidoni, \& Betowski, 2000) demonstrate also preferential protonation on a carbon of the ring(s). The role of the substitutent is to orient protonation on specific sites, for example in para and ortho positions when only a phenyl ring is present in the molecule.

A substituent bearing a lone-pair placed on a CC double or triple bond provide also a possibility of resonance effect but may also offer a preferential basic site. This is observed for electronattractor substituents such as $\mathrm{CN}, \mathrm{CHO}$ or $\mathrm{NO}_{2}$ but also for some electron-donor groups. The simplest cases are $\mathrm{CH}_{2}=\mathrm{CHX}$ and HCCX (X=elements of groups 15-17). Molecular orbital calculations demonstrate that the favored protonation sites of these molecules are the $\beta$-carbon atom for $X=N, O, S$ and the heteroatom when $\mathrm{X}=\mathrm{P}$; intermediate situation is established for $\mathbf{X}=$ As (Mo et al., 1999). At the G2 level, structures $\mathbf{e C}$ and $\mathbf{y C}$ are favored over $\mathbf{e X}$ and $\mathbf{y X}$ (Scheme 8) by 15 and $24 \mathrm{~kJ} \mathrm{~mol}^{-1}$ for $\mathrm{X}=\mathrm{N}$ (Mo et al., 1999; see also Smith \& Radom, 1992 for another theoretical level), by 96 and $173 \mathrm{~kJ} \mathrm{~mol}^{-1}$ for $\mathrm{X}=\mathrm{OH}$ and by 54 and $100 \mathrm{~kJ} \mathrm{~mol}^{-1}$ for $\mathrm{X}=\mathrm{SH}$ (this work; see also Fairley et al., 1996 and Petrie, 2005 for similar calculations on $\boldsymbol{e C}$ and $\boldsymbol{e} \boldsymbol{X}$ ions with $\mathrm{X}=\mathrm{OH}$ ), while the situation is reversed for
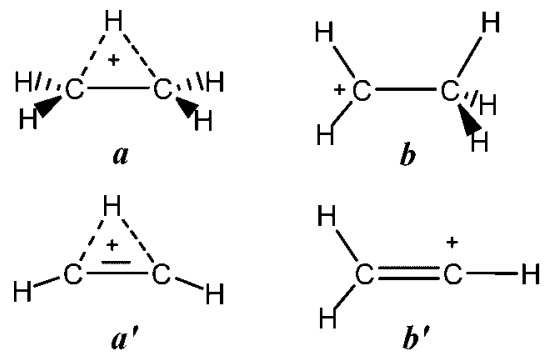

SCHEME 6.
$\mathrm{X}=\mathrm{P}$ with $\mathbf{e X}$ and $\mathbf{y X}$ being more stable than $\mathbf{e C}$ and $\mathbf{y C}$ by 11 and $7 \mathrm{~kJ} \mathrm{~mol}^{-1}$ (Mo et al., 1999).

Similar effects are also present in heteroaromatic molecules which may be protonated either on the $\pi$-electron system or on a non-bonding electron pair. To take two examples, furan and pyrrole show a clearcut preference for protonation at the carbon in position 2, the decreasing order of preference of the other possible protonation sites is the carbon 3 and the heteroatom. The preference for protonation at position 2 can be rationalized in term of resonance structures. Accordingly, the carbon 2 protonated structures have three resonance structures whereas the carbon 3 protonated form has only two. Protonation at the heteroatom is least favorable because it results in a formal localization of the charge on the heteroatom without possibility of its resonance delocalization. Relative $298 \mathrm{~K}$ enthalpies, in $\mathrm{kJ} \mathrm{mol}^{-1}$, of the various protonated forms of furan and pyrrole, calculated at the G3 level, are summarized in Scheme 9 (Kabli et al., 2006; see also Chan et al., 2005 for complementary calculations at a lower level of theory).

By contrast, protonation of pyridine unambiguously occurs on the nitrogen atom since its lone pair is not involved in the $\pi$-electron delocalization. Relative energies of the various protonated forms of pyridine indicated in Scheme 9 results from B3LYP/6-311G(2d,p) enthalpy calculations at 298 K (Nguyen \& Turecek, 1997; Pham-Tran et al.).

\section{Substituents Effects on Protonation}

At variance with solution chemistry where it is often difficult to separate solvent and substituent effects, gas-phase chemistry allows the understanding of intrinsic reactivity. Substituent effect on thermochemical properties of gaseous cations and anions has been consequently a subject of intense interest for a long time (Taft \& Topsom, 1987; Gal \& Maria, 1990; Chuchani et al., 1999). The substituent effects are generally interpreted by the combination of terms taking into account (i) the electrostatic effects (field/inductive, polarizability) and (ii) the resonance of $\pi$ electrons. The first effects occur without transfer of electron from or to the substituent whereas resonance obviously involves this
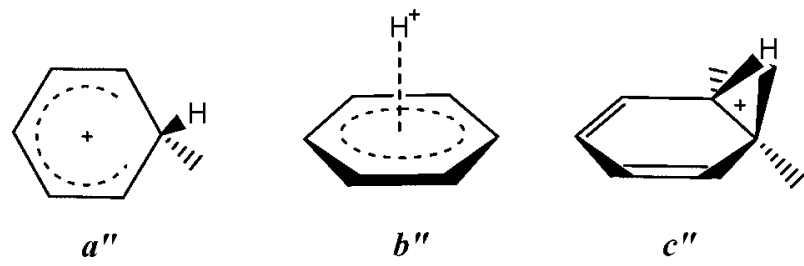

SCHEME 7. 
<smiles>[X]C=C</smiles>

$e$

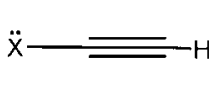

$y$

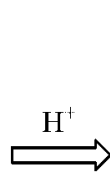<smiles>[X]CC</smiles><smiles>[Y1]C=C</smiles><smiles>[X]C#C[3H]</smiles>

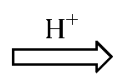

$$
x
$$

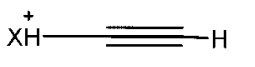

$e C$

$\mathrm{X}=\mathrm{N}, \mathrm{O}, \mathrm{S}(\mathrm{As})$

eX $\quad \mathrm{X}=\mathrm{P}(\mathrm{As})$

$y C$

$\mathrm{X}=\mathrm{N}, \mathrm{O}, \mathrm{S}(\mathrm{As})$

$\boldsymbol{y} \boldsymbol{X}$

$\mathrm{X}=\mathrm{P}(\mathrm{As})$

\section{SCHEME 8.}

kind of transfer. Finally, steric effects may be invoqued in certain circumstances.

The general treatment of substituent effects supposes that it is possible to separate the various types of interactions between a substituent (a small part of the molecule which quantitatively influences its properties but does not change its general characteristics) and a reaction site (a functional group, for example a basic site) separated from the substituent by the remaining framework of the molecule. As far as protonation thermochemistry is concerned (i.e., the thermodynamic of reaction (1): $\mathrm{MH}^{+} \rightarrow \mathrm{M}+\mathrm{H}^{+}$), substituent effects on both the neutral molecule and its protonated form should be envisaged. Due to the exacerbation of intramolecular electrostatic interaction in positively charged species and to the possibility of charge stabilization by $\pi$-electrons resonance, stabilization due to the various forms of the substituent effects is generally greater for the protonated molecule $\mathrm{MH}^{+}$than for the neutral $\mathrm{M}$. This is why the contribution of the neutral is often neglected to qualitatively estimate the substituent effect on gas-phase basicity and proton affinity. Obviously, a quantitative treatment of protonation thermochemical data should include consideration of electrostatic interactions and resonance effects in both the neutral and the protonated base. A brief recall of the definitions relevant to the electrostatic effects expected to be produced by substituents bearing a permanent dipole or a polarizable moiety on a positive charge or on a permanent dipole is first presented.

a. Electrostatic effects. If we consider a point charge $q$ situated at a distance $r$ from the center of a locked dipole $\mu$, the interaction
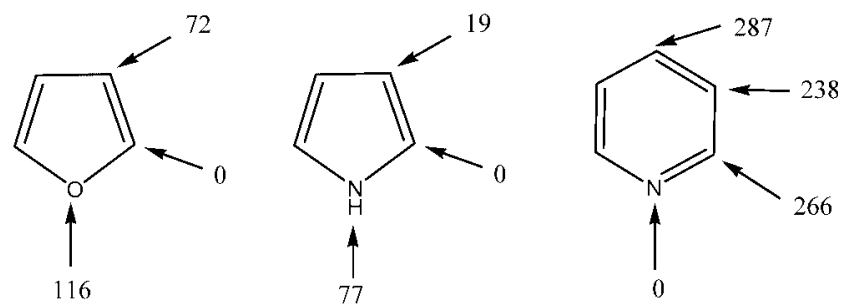

SCHEME 9. energy due to charge-dipole electrostatic forces is given by

$$
E_{\mathrm{Fcd}}=\frac{q \mu \cos \theta}{4 \pi \varepsilon r^{2}}
$$

where $\theta$ is the angle between the dipole and the point charge and $\varepsilon$ is the effective permittivity (Scheme 10). This through space (or "field") interaction may schematically describes the effect of a polar substituent on the charge of a protonated molecule. This effect operates at relatively long range via the $1 / r^{2}$ relationship and, according to Equation (94), its sign (it may be either attractive or repulsive) and intensity depends drastically on the orientation $\theta$ of the dipole, the maximum stabilization being $\left(E_{\mathrm{Fcd}}\right)_{\max }=q \mu / 4 \pi \varepsilon r^{2}$.

The influence of a polar substituent $\left(\mu_{\mathrm{A}}\right)$ on a second dipole $\left(\mu_{\mathrm{B}}\right)$ may be similarly described (Scheme 10$)$. In that case, the potential energy of interaction takes the form:

$$
E_{\mathrm{Fdd}}=\left(\frac{\mu_{\mathrm{A}} \mu_{\mathrm{B}}}{4 \pi \varepsilon r^{3}}\right)\left(2 \cos \theta_{\mathrm{A}} \cos \theta_{\mathrm{B}}-\sin \theta_{\mathrm{A}} \sin \theta_{\mathrm{B}} \cos \phi\right)
$$

where $\phi$ represents the dihedral angle between the two planes containing each dipole and the $r$ line. Equation (95) represents a medium range interaction which takes its maximum value, $\left(E_{\mathrm{Fdd}}\right)_{\max }=-2 \mu_{\mathrm{A}} \mu_{\mathrm{B}} / 4 \pi \varepsilon r^{3}$, for $\theta_{\mathrm{A}}=0^{\circ}$ and $\theta_{\mathrm{B}}=180^{\circ}$, that is, when the dipoles are collinear. Another particular configuration is the antiparallel arrangement $\left(\theta_{\mathrm{A}}=\theta_{\mathrm{B}}=90^{\circ}\right)$ which leads to a stabilization energy $E_{\mathrm{Fdd}}$ of $-\mu_{\mathrm{A}} \mu_{\mathrm{B}} / 4 \pi \varepsilon r^{3}$, that is, $\left(E_{\mathrm{Fdd}}\right)_{\max } / 2$.

If we replace the polar substituent by a polarizable entity of polarizability $\alpha$ (Scheme 11), the point charge will be at the origin of an induced dipole $\mu^{\prime}$ and consequently to an attractive force. In this simplest view the interaction energy takes the form:

$$
E_{\alpha^{c p}}=\frac{-\alpha q^{2}}{2(4 \pi \varepsilon)^{2} r^{4}}
$$

Formation of an induced dipole $\mu^{\prime}$ is also possible from the interaction between the polarizable substituent and a dipolar functional group (Scheme 11). The resulting energy gain is then given by Equation (97):

$$
E_{\alpha \mathrm{dp}}=-\left(\frac{\alpha \mu^{2}}{2(4 \pi \varepsilon)^{2} r^{6}}\right)\left(1+3 \cos ^{2} \theta\right)
$$



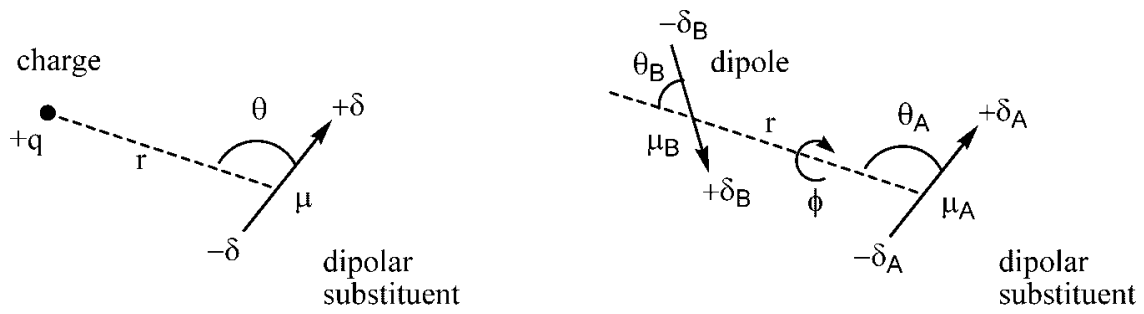

(a) field effect

SCHEME 10.

Obviously, the maximum stabilization is expected for $\theta=0$ which leads to $\left(E_{\alpha^{\mathrm{dp}}}\right)_{\max }=-4 \alpha \mu^{2} / 2(4 \pi \varepsilon)^{2} r^{6}$.

Considering the role of $r$ in Equations (94)-(97), it appears that the polarizability effect is efficient at a shorter distance than the field effect. Illustration of the role of the distance $r$ on the various contributions to electrostatic interactions is given in Figure 25. For these examples, the dipole moments $\mu$ and the polarizability $\alpha$ have been taken equal to 5 Debye and $5 \AA^{3}$, respectively, and the angles $\theta$ chosen to obtain the maximum stabilization energies. The effective permittivity of the medium $\varepsilon$ has to be taken equal to $\varepsilon_{0}$ the permittivity of the vacuum $\left(\varepsilon_{0}=8.854 \times 10^{-12} \mathrm{C}^{2} \mathrm{~J}^{-1} \mathrm{~m}^{-1}\right)$. It is interesting also to compare the absolute stabilization energy values at fixed distance $r$. For example $\left(E_{\mathrm{Fcd}}\right)_{\max }=-90.4,\left(E_{\mathrm{Fdd}}\right)_{\max }=-47.1,\left(E_{\alpha^{\mathrm{dp}}}\right)=-13.6$ and $\left(E_{\alpha \mathrm{dp}}\right)_{\max }=-7.4 \mathrm{~kJ} \mathrm{~mol}^{-1}$ for $r=4 \AA$, showing a decreasing order of importance of the various contribution.

The two electrostatic effects, field and polarizability, are dependent on the permittivity of the surrounding and it is therefore apparent that in solvent of high dielectric constant the incidence of $E_{\mathrm{F}}$ and $E_{\alpha}$ is reduced, while it is amplified in the gas phase.

If the importance of electrostatic interactions on the stability and reactivity of isolated ions should be emphasized, their influence on the properties of the neutral counterpart should not be forgotten either. It may be simply recalled that the dissociation energy of a heteronuclear bond $\mathrm{AB}$ is generally larger than the average of the bond dissociation energies of AA and BB. Since Pauling (1960), this extra stabilization is described as resonance between the covalent and ionic structures $\boldsymbol{a}-\boldsymbol{c}$ (Scheme 12).

The extent of resonance depends on the stability of the ionic configurations which is related to the ability of A or B to attract electron. As it is well known, this idea forms the basis of the concept of electronegativity and appears to explain most of the chemical bonds characteristic in the periodic system (Mo et al., 2005).

The situation is more delicate when polarizable substituents (mostly hydrocarbon groups) are involved. Effect of alkyl substituents $\mathrm{R}\left(\mathrm{R}=\mathrm{CH}_{3}, \mathrm{C}_{2} \mathrm{H}_{5}, i-\mathrm{C}_{3} \mathrm{H}_{7}\right.$ and $\left.t-\mathrm{C}_{4} \mathrm{H}_{9}\right)$ on homolytic bond dissociation energies of RX molecules has been shown to depend strongly on $\mathrm{X}$ (Coote et al., 2003). For $\mathrm{X}=\mathrm{H}$ and, to a less extend, $\mathrm{X}=\mathrm{CH}_{3}$, a decrease of bond dissociation energies is noted with increasing alkylation (i.e., when going from $\mathrm{R}=\mathrm{CH}_{3}$ to $\mathrm{R}=t-\mathrm{C}_{4} \mathrm{H}_{9}$ ). For $\mathrm{X}=\mathrm{F}, \mathrm{OH}$, and $\mathrm{OCH}_{3}$, the reverse is observed: an increase of the bond dissociation energies accompanies the increasing alkylation. Interestingly, the $\mathrm{R}-\mathrm{X}$ bond length always increases, whatever the nature of $\mathrm{X}$, when the size of the alkyl group increases. A simple explanation is provided by a simplified valence bond description involving structures $\boldsymbol{a}^{\prime}$ and $\boldsymbol{b}^{\prime}$ (Scheme 13).

It must be first underlined that, in this conceptual view, the mixing between the ionic and covalent limiting structures $\mathbf{a}^{\prime}$ and $\mathbf{b}^{\prime}$ leads to a stabilization of the resonance hybrid. It is consequently expected that the $\mathrm{R}-\mathrm{X}$ bond dissociation energy will increases when the mixing between $\mathbf{a}^{\prime}$ and $\mathbf{b}^{\prime}$ increases. The second point is that the participation of the limiting structure $\mathbf{a}^{\prime}$ is heavier for electronegative $\mathrm{X}$, thus the mixing is more important for $\mathrm{X}=\mathrm{F}$ and is decreasing for $\mathrm{X}=\mathrm{OH}$ and $\mathrm{OCH}_{3}$. The consequence is the well-established results that, for a given $\mathrm{R}$, the $\mathrm{R}-\mathrm{X}$ bond dissociation energy increases with increasing $\mathrm{X}$ electronegativity. The third point to consider, is that the limiting structure $\mathbf{a}^{\prime}$ will be stabilized by an alkyl group R to the extend of the polarizability of this latter. Consequently, we expect the stability of $\mathbf{a}^{\prime}$, and thus that of the resonance hybrid, to increase in
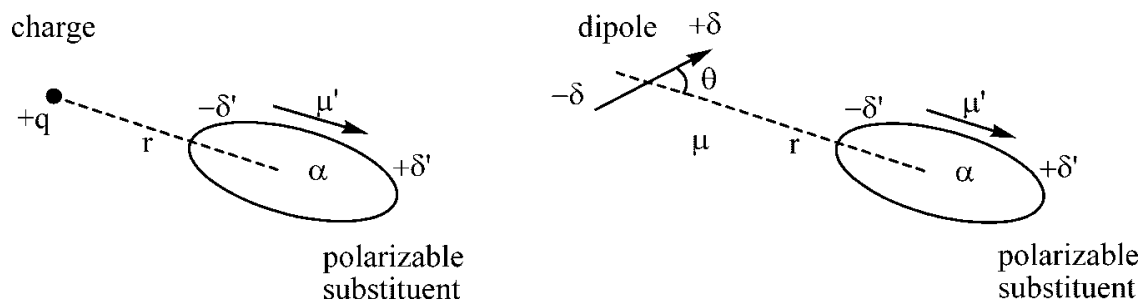

(b) polarizability effect

SCHEME 11. 


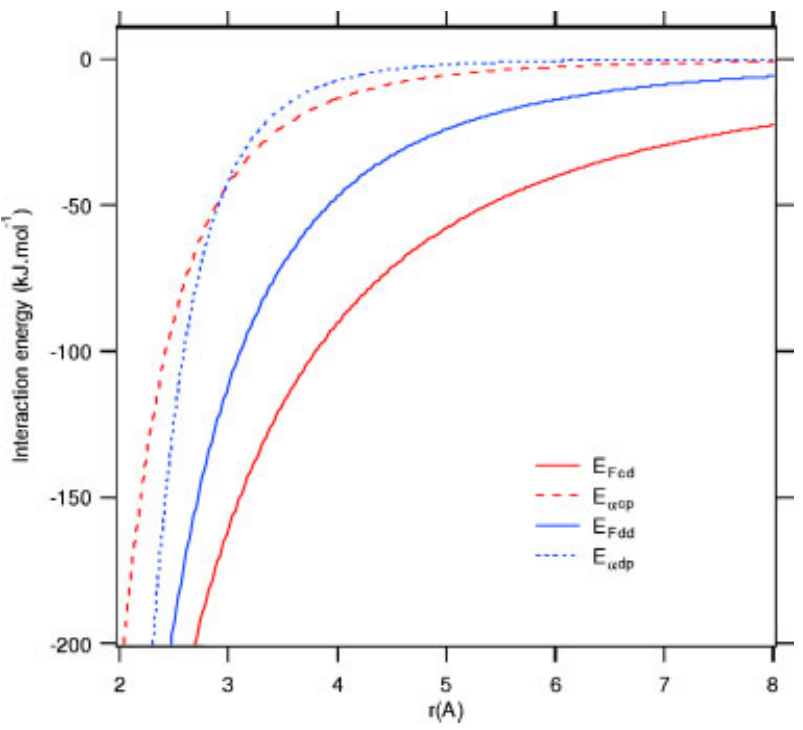

FIGURE 25. Stabilization energies afforded by charge-dipole $\left(E_{\mathrm{Fcd}}\right)$, charge-polarizable moiety $\left(E_{\alpha^{\text {cp }}}\right)$, dipole-dipole $\left(\mathrm{E}_{\mathrm{Fdd}}\right)$ and dipolepolarizable $\left(E_{\alpha^{\mathrm{dp}}}\right)$ moiety interactions as a function of the distance $r$, for a typical situation where $\mu=5$ Debye and $\alpha=5 \AA^{3}$. [Color figure can be viewed in the online issue, which is available at www.interscience. wiley.com.]

the order $\mathrm{CH}_{3}<\mathrm{C}_{2} \mathrm{H}_{5}<i-\mathrm{C}_{3} \mathrm{H}_{7}<t-\mathrm{C}_{4} \mathrm{H}_{9}$. The combined stabilizing effects experienced by the RX molecules obviously account for the increase in the $\mathrm{R}-\mathrm{X}$ bond dissociation energies with increasing both alkylation and $\mathrm{X}$ electronegativity. It may be finally noted that, in addition to electrostatic effects, alkyl groups may also participate to the bonding properties of neutral and ionized species by hyperconjugative electron donation (Mayer et al., 1997). This does not change the above reasoning.

b. Resonance effect. The substituent resonance effect arises when the substituent participates to the transfer of $\pi$ electrons from, or to, the probe center (Scheme 14).

It is a well known effect, familiar to chemists who separate substituents containing an atom or group of atoms with a lone pair: the $\pi$-electron donors substituents, and substituents containing unsaturated groups which are generally $\pi$ electron acceptors. Transmission of the effect in an extended $\pi$ system may occur at very long range without significant alteration. As underlined in a preceding paragraph, resonance is responsible of considerable stabilization of the protonated molecules by charge delocalization which results in an increase in proton affinities. The resonance effect acts also in the orientation of the protonation on the site leading to the largest delocalization of the charge (see for example Schemes 7-9).

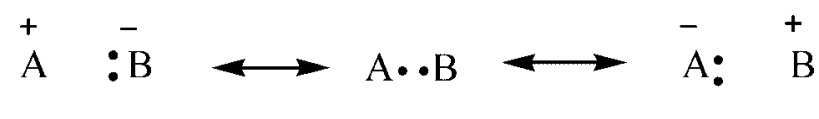
$a$

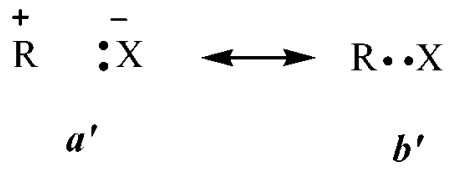

SCHEME 13.

c. Linear free energy relationship. A quantitative, but empirical, measure of substituent effects has been originally stated by Hammett 70 years ago (Hammett, 1937) who shows that the effects of a substituent $X$ on the rate or equilibrium constants of reactions of benzene derivatives takes the form:

$$
\ln \left(\frac{K_{\mathrm{X}}}{K_{\mathrm{H}}}\right)=\rho \sigma_{\mathrm{X}}
$$

where $\rho$ is specific of a given reaction and $\sigma_{\mathrm{X}}$ a constant expressing the substituent $X$ effect. Linear free energy relationship given by Equation (98) has been successful in treating the substituent effects of a large variety of solution and gas-phase reactions (Harrison, 1999). The generalized treatment proposed by Taft and Topsom (1987) depasses the frame of aromatic compounds to describe the case of alphatic molecules. The authors introduce a summation of three distinct contributions, including field, polarizability and resonance effects, such as

$$
\Delta G^{0}=\rho_{\mathrm{F}} \sigma_{\mathrm{F}}+\rho_{\alpha} \sigma_{\alpha}+\rho_{\mathrm{R}} \sigma_{\mathrm{R}}
$$

to express the difference in free energy between the considered reaction occurring with a substituent $X$ and the corresponding reaction with unsubstituted reactant.

Applicability of Equation (99) to protonation thermochemistry may be illustrated by the correlation observed between the gas-phase basicity of monodentate aliphatic bases with the polarizability index $\sigma_{\alpha}$ of Taft (Fig. 26). A linear relationship is indeed observed between $\Delta \mathrm{GB}=\mathrm{GB}(\mathrm{XH})-\mathrm{GB}(\mathrm{XR})\left(\mathrm{X}=\mathrm{NH}_{2}\right.$ and $\mathrm{OH})$ and $\sigma_{\alpha}$. The significant increase of the gas-phase basicity with the size of the alkyl group $\mathrm{R}$ means that $\mathrm{R}$ stabilizes more efficiently the protonated forms than the neutral bases. This is clearly in line with the (charge/induced dipole) stabilizing effect of an alkyl group $\mathrm{R}$ on a positive charge presented above. A comparison between the two correlation lines corresponding to $\mathrm{RNH}_{2}$ and $\mathrm{ROH}$ series in Figure 26 reveals different slopes $\rho_{\alpha}$ (Eq. (99)). The stronger increase in basicity observed for the later series may be explained by the larger electron demand from an oxonium ion compared to an immonium center, or, to return to

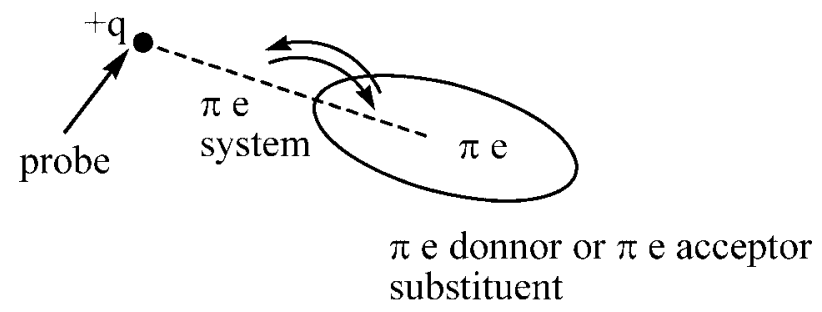

(c) resonance effect SCHEME 14. 


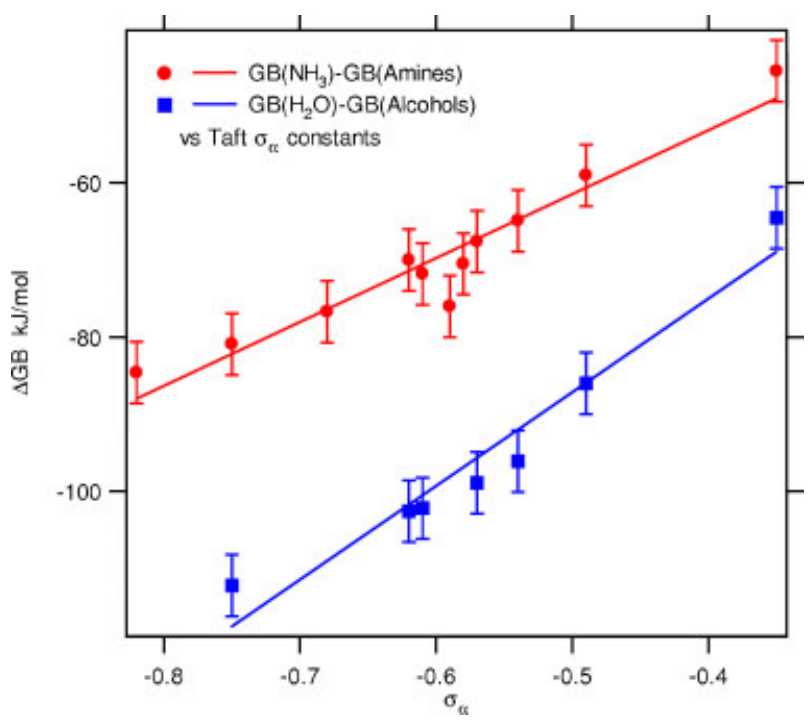

FIGURE 26. Correlation between proton affinity of primary amines $\mathrm{RNH}_{2}$ and alcohols ROH with the Taft $\sigma_{\alpha}$ constant of substituents $R$. [Color figure can be viewed in the online issue, which is available at www.interscience.wiley.com.]

Scheme 2, by a larger participation of limiting structure $\mathbf{1 H i}$ in the description of the oxonium ions.

The role of field and resonance effect may be illustrated by the protonation of monosubstituted benzenes. The correlation of the proton affinities of $\mathrm{C}_{6} \mathrm{H}_{5} \mathrm{X}$ molecules with the Brown constant $\sigma_{\text {para }}^{+}$is presented in Figure 27. Points corresponding to electron withdrawing substituents $\mathrm{CO}_{2} \mathrm{CH}_{3}, \mathrm{CN}$, and $\mathrm{NO}_{2}$ have clearly different behavior that the other substituents. An excellent linear correlation is found $(\mathrm{Pr}=0.98)$ if we exclude the three abovementioned substituents of the correlation. The explanation in that, except for $\mathrm{CO}_{2} \mathrm{CH}_{3}, \mathrm{CN}$ and $\mathrm{NO}_{2}$, protonation occurs in para position of the aromatic ring and the constant $\sigma_{\text {para }}^{+}$is well suited

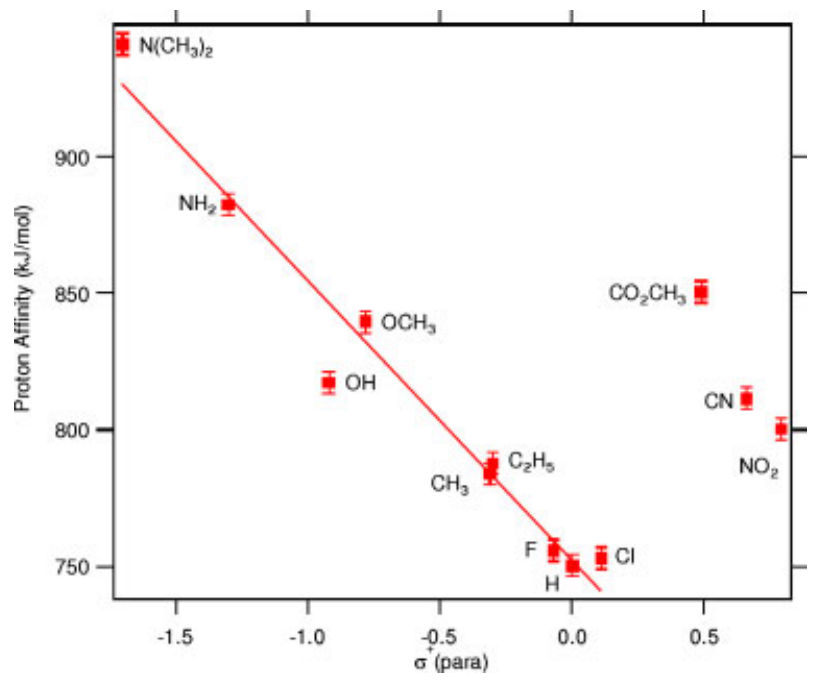

FIGURE 27. Correlation of proton affinities of monosubstituted benzenes with $\sigma_{\text {para }}^{+}\left(\mathrm{CO}_{2} \mathrm{CH}_{3}, \mathrm{CN}\right.$, and $\mathrm{NO}_{2}$ are omitted from the correlation). [Color figure can be viewed in the online issue, which is available at www.interscience.wiley.com.] to describe the stabilization of a positive charge by $\pi$-electron donor effect. Deviation from linear free energy relationship may be related to a change in reactivity, or of structure, in the series. In the present case, the deviation observed for the $\pi$-electron attractor substituents $\mathrm{CO}_{2} \mathrm{CH}_{3}, \mathrm{CN}$, and $\mathrm{NO}_{2}$ is due to the fact that protonation occurs on the substituent as described in Introduction.

Note however that protonation on the substituent may accidentally correspond to a proton affinity similar to the para position. This seems to be the case for aniline as indicated by recent molecular orbital calculations which point to very similar energies for the $N$ and para protonated aniline (Russo et al., 2000).

d. Correlating proton affinities with ionization energies. In assessing protonation thermochemistry in the gas phase, it has proven useful to establish empirical correlations between proton affinities and some other properties of the considered molecules. In line with this goal, several approaches have been developed for relating proton affinity with valence electron (Staley \& Beauchamp, 1974; Aue, Webb, \& Bowers, 1976; Staley, Kleckner, \& Beauchamp, 1976; Campbell et al., 1992) or core electron (Davis \& Rabalais, 1974; Martin \& Shirley, 1974; Caroll et al., 1974; Mills, Martin, \& Shirley, 1976; Benoit \& Harrison, 1977; Harrison, 1999) ionization energies. The basic idea starts from the simple observation that proton affinity, PA, and ionization energy IE, in both cases depend on differences in energy between species which essentially differ in charge. Moreover, to remove an electron and to add a proton are electrically analogous processes. Trends in both quantities, PA and IE, are related to the interplay of inductive and polarization effects in the neutral and in the positively charged state (see Characteristics Related to the Nature of the Basic Site). Introduction of a positive charge, either by protonation of by removal of one electron, is accompanied by an energy difference that varies mainly through the ability to stabilize the charge. Based on a simple model two groups (Davis \& Rabalais, 1974; Martin \& Shirley, 1974) independently showed that a linear correlation of unit slope should exist between proton affinity and inner-shell electron binding energies within a homologous series of molecules. This model was expected to interpret the alkyl group effect mainly due to its polarizability which stabilizes more efficiently the charged species. Linear correlations between PA and core-electron energies, $\mathrm{IE}_{\mathrm{core}}$, were indeed observed for series of alcohols, ethers, amines, and carbonyl molecules (Davis \& Rabalais, 1974; Martin \& Shirley, 1974; Mills, Martin, \& Shirley, 1976; Benoit \& Harrison, 1977; Slaughter \& Banna, 1988), however, separate correlation were observed for different functionalities (e.g., alcohols and ethers; primary, secondary or tertiary amines). Similar results have been finally obtained by using valence shell electron ionization energy, $\mathrm{IE}_{\mathrm{valence}}$, rather than $\mathrm{IE}_{\text {core }}$ (Staley \& Beauchamp, 1974; Aue, Webb, \& Bowers, 1976; Staley, Kleckner, \& Beauchamp, 1976; Benoit \& Harrison, 1977). For example, nitriles proton affinities are found to be linearly correlated to the adiabatic $\mathrm{N}$ lone pair ionization energy and not to the first ionization energy which is related to a $\pi_{\mathrm{CN}}$ orbital (Staley \& Beauchamp, 1974). One interest of this kind of correlation is its ability to predict, or verify, the protonation site of a given molecule or set of molecules. As an example, the linear 
correlation between the PA of primary amines of general formula $\mathrm{RCH}_{2} \mathrm{NH}_{2}\left(\mathrm{R}=\mathrm{H}, \mathrm{CH}_{3}, \mathrm{C}_{2} \mathrm{H}_{5}, \mathrm{C}_{3} \mathrm{H}_{7}, \mathrm{C}_{4} \mathrm{H}_{9}, \mathrm{C}_{5} \mathrm{H}_{11}\right.$, and $\left.\mathrm{C}_{7} \mathrm{H}_{15}\right)$ is presented in Figure 28. All energetic values are taken from the available compilations: (Hunter \& Lias, 1998) for PA and (Lias et al., 1988) for IE, the assumed errors bars correspond to $\pm 4 \mathrm{~kJ} \mathrm{~mol}^{-1}$ and $0.02 \mathrm{eV}$ for PA and IE, respectively. From least square analysis, the fitting line corresponds to the equation $\mathrm{PA}\left(\mathrm{kJ} \mathrm{mol}^{-1}\right)=1477.7-64.03 \mathrm{EI}(\mathrm{eV})$ with a correct correlation coefficient $r=0.956$. The points, PA versus $\mathrm{IE}_{\mathrm{valence}}$, of three bifunctional molecules are also presented in the same graph. The large discrepancies observed undoubtly show that the stabilization of the charged species are completely different for these molecules than for the primary amines. Accordingly, the formation of a strong internal hydrogen bond in protonated diamine and aminoalcohols overshadow the polarizability effect responsible of the linear behavior of primary amines.

In a similar way, using also primary amines as reference species, Beauchamp and co-workers (Campbell et al., 1992) find a linear correlation between the PA and the nitrogen lone pair ionization energies of most of the aminoacids which confirms the amine group as the most favorable protonation site. Deviation from the correlation is observed for aminoacids able to form strong intramolecular hydrogen bonds (e.g., tryptophane) or which protonate preferentially on the side chain (e.g., arginine).

The recently proposed dissection of the protonation process into ionization of the base, formation of the $\mathrm{H}$ atom with the incoming proton and homolytic bond formation between $\mathrm{H}$ and the radical cation of the base, may be seen as an extension of the above discussed correlations (Maksic \& Vianello, 2002).

e. Correlating proton affinity with the size of the molecule. A number of thermochemical properties follow additivity rules and

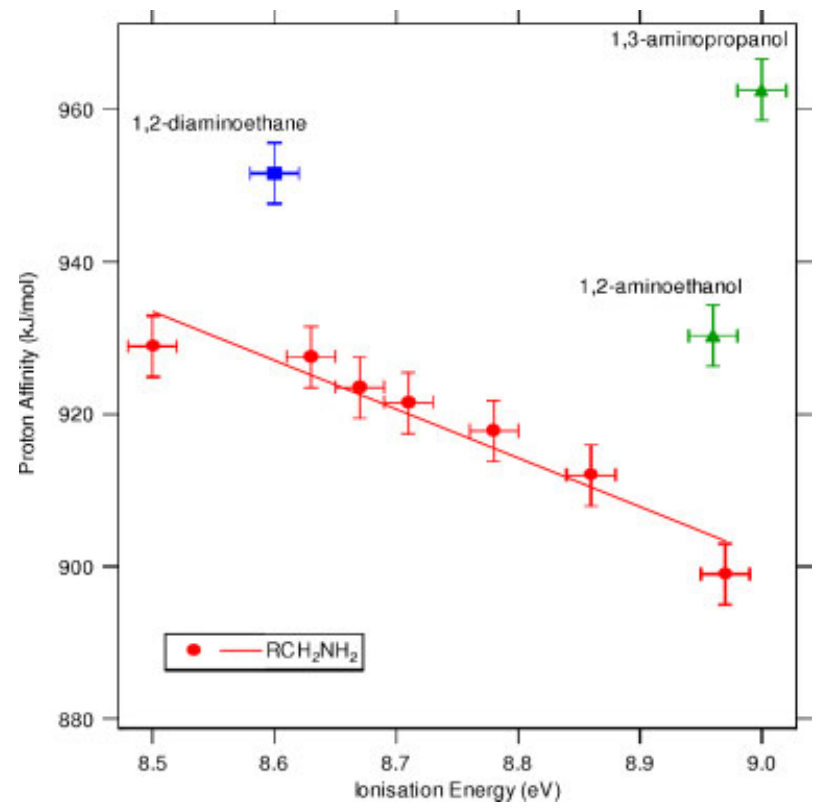

FIGURE 28. Example of linear correlation between proton affinity and valence shell ionization energy: primary amines of general formula $\mathrm{RCH}_{2} \mathrm{NH}_{2}$ and several substituted amines. [Color figure can be viewed in the online issue, which is available at www.interscience.wiley.com.] thus presents linear dependence with the number of groups, or bonds or atoms of the molecule. Among these quantities are standard heats of formation, heat capacities and third law entropies of neutral species (Benson, 1976). By contrast, this is not the case for thermochemical quantities involving charged species: heats of formation of ions, ionization energies and proton affinities show evolutions decreasing in rate along a homologous series defined by the sequential change in methylene groups. Clearly, the ability to stabilize a charge does not follow a linear relationship with the size of the considered species but rather, an asymptotic law. Holmes and co-workers show that ionization energies and proton affinities follow a linear relationship with $1 / n$, where $\mathrm{n}$ is the number of atoms of the molecule (Holmes \& Lossing, 1991; Aubry \& Holmes, 2000; Holmes, 2003). Heats of formation of cations may be also expressed via a $1 / n$ law (Holmes, Fingas, \& Lossing, 1981) or a $\ln (n)$ relationship (Holmes \& Lossing, 1982). Different correlation lines are obtained for different functional groups and the slope of the IE (or $\Delta_{\mathrm{f}} H^{0}$ (ion)) vs. $1 / n$ (or $\ln (n)$ ) lines seems to decrease in the order alkane $>$ alcohol-ether $>$ chloride $>$ bromide $>$ iodide.

Figure 29 presents an example of linear correlation between proton affinities and $1 / n$ for a series of primary amines $\mathrm{RCH}_{2} \mathrm{NH}_{2}$. Al the energetic values were taken from the Hunter \& Lias compilation (Hunter \& Lias, 1998) except for $n$-heptylamine for which the proton affinity value has been readjusted to $928.0 \mathrm{~kJ} \mathrm{~mol}^{-1}$. The linear fitting is excellent $(\operatorname{Pr}=0.998)$ and the decrease of proton affinity with decreasing the size of the molecule is in line with the parallel decrease in polarizability of the substituent R. In connection with this remark, it should be underlined that polarizability is linearly related to the number of atoms or electrons of the considered species (Miller, 1990). A clear illustration is this relationship is given in Figure 30 where the polarizability of a series of alkane and of some amines is plotted against the number of atoms (solid line) or the number of electrons (dashed line).

In Figure 29 are also presented data for two series of bifunctional bases bearing also an amino group: the diamines

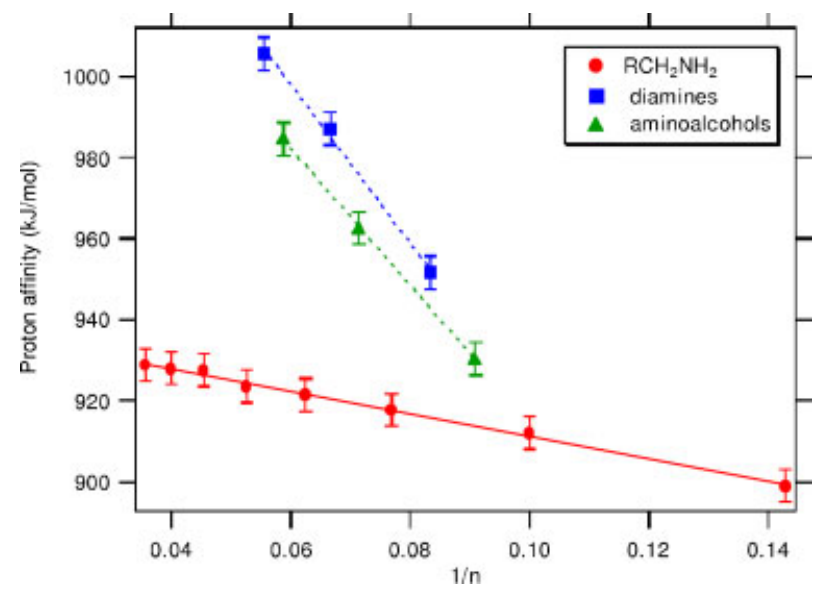

FIGURE 29. Example of linear correlation between proton affinity and the size of the molecule: primary amines of general formula $\mathrm{RCH}_{2} \mathrm{NH}_{2}$ $\left(\mathrm{R}=\mathrm{H}, \mathrm{CH}_{3}, \mathrm{C}_{2} \mathrm{H}_{5}, \mathrm{C}_{3} \mathrm{H}_{7}, \mathrm{C}_{4} \mathrm{H}_{9}, \mathrm{C}_{5} \mathrm{H}_{11}, \mathrm{C}_{6} \mathrm{H}_{13}\right.$, and $\left.\mathrm{C}_{7} \mathrm{H}_{15}\right)$, diamines $\mathrm{NH}_{2}\left[\mathrm{CH}_{2}\right]_{p} \mathrm{NH}_{2}$ and aminoalcohols $\mathrm{NH}_{2}\left[\mathrm{CH}_{2}\right]_{p} \mathrm{OH}(p=2,3$, and 4). [Color figure can be viewed in the online issue, which is available at www.interscience.wiley.com.] 


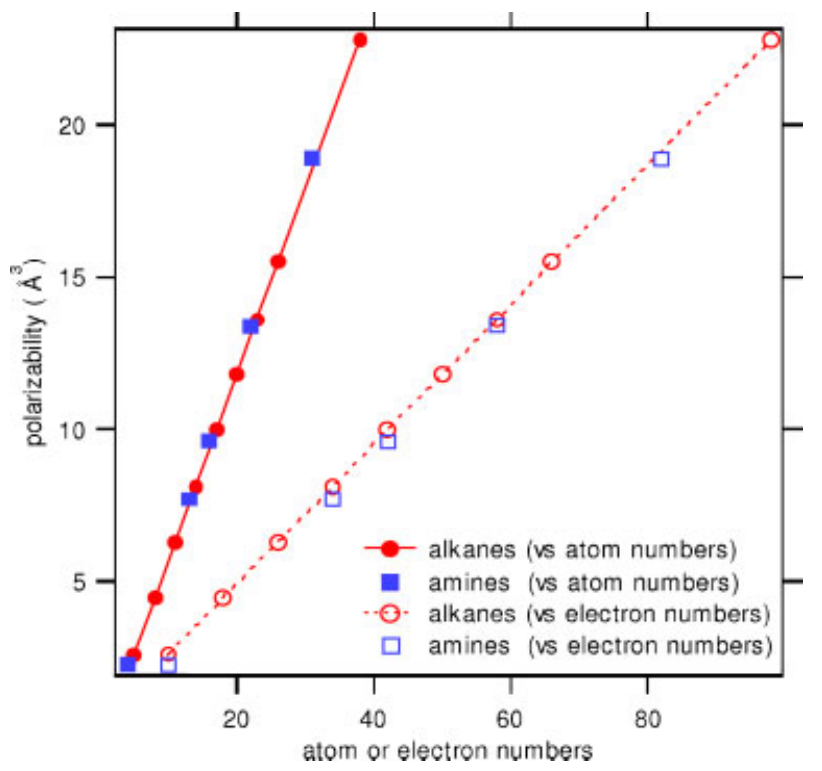

FIGURE 30. Correlation between molecular polarizabilities and the number of electrons or atoms in the molecule. [Color figure can be viewed in the online issue, which is available at www.interscience.wiley.com.]

$\mathrm{NH}_{2}\left[\mathrm{CH}_{2}\right]_{p} \mathrm{NH}_{2}$ and aminoalcohols $\mathrm{NH}_{2}\left[\mathrm{CH}_{2}\right]_{p} \mathrm{OH}(p=2,3$, and 4). It is remarkable that, even for these species, a linear fit is also observed. The correlation lines are however clearly different from that of the monofunctional bases indicating that the change in the number of methylene groups is associated with a different stabilizing effects on the protonated bases. Accordingly, the $1 / n$ term is also related to the number of carbon atoms separating the functional groups, a major parameter in the stabilization of protonated bifunctional molecules by intramolecular hydrogen bonds as it will be emphasized in a next section (see Intramolecular Hydrogen Bonding). The observed linear behavior is thus not related to polarizability but to another electrostatic interaction which also correlate with $1 / n$.

\section{Hydrogen Bonding and Protonation}

Hydrogen bonding is an important intermolecular and intramolecular interaction encountered in neutral or ionized systems, in gas, liquid, and solid phases. It plays a very crucial role in many chemical and biochemical processes and in the protonation events since it may be considered as the first step of a proton transfer. In a large sense, a hydrogen bond involves a hydrogen atom covalently bound to an electron deficient center, A-H (or $\mathrm{A}-\mathrm{H}^{+}$), and an acceptor center $\mathrm{B}$ (or $\mathrm{B}^{-}$) including a nonbonding lone pair or a $\pi$-electrons system (Jeffrey, 1997; Scheiner, 1997; Gilli \& Gilli, 2000; Sobczyk, Grabowski \&, Krygowski, 2005). The proton donor part A-H contains classically an electronegative atom but weak donors systems, such as certain $\mathrm{C}-\mathrm{H}, \mathrm{P}-\mathrm{H}, \ldots$ bonds, have been also identified and theoretically characterized (Scheiner, 1997; Desiraju \& Steiner, 1999; Hartmann, Westmore, \& Radom, 2001; Wetmore et al., 2001; El Firdoussi et al., 2005). To understand the consequences of hydrogen bonding in the basicity of polyfunc- tional compounds it is of interest to recall some results pertaining to bimolecular complexes, both neutral and protonated, bounded together by a hydrogen bond.

\section{Intermolecular Hydrogen Bonding}

a. Neutral hydrogen bonded dimers. A large number of neutral hydrogen bonded dimers $\mathrm{A}-\mathrm{H} \cdots \mathrm{B}$ have been investigated by rotational spectroscopy (Legon \& Millen, 1987; Jeffrey, 1997). Because this experimental method is conducted in the gas-phase under low pressure, the derived structural and energetic informations refer to isolated complexes. On the basis of these experimental data, rules concerning the geometries of hydrogen bonded complexes have been enunciated (Legon \& Millen, 1987). These rules state that the axis of the AH molecule (i) coincides with the axis of the non-bonding electron pairs of $\mathrm{B}$, or, if the interaction involve $\pi$-electrons rather than non-bonding electrons, (ii) is perpendicular to the plane of the $\pi$-electrons system. Following Jeffrey in his large compilation involving both condensed and gas-phase data (Jeffrey, 1997), weak to moderate hydrogen bonds between $\mathrm{AH}$ and $\mathrm{B}$ are essentially electrostatic and the $\mathrm{A}-\mathrm{H} \cdots \mathrm{B}$ complexes are characterized by $\mathrm{H} \cdots \mathrm{B}$ distances in the 2-3 $\AA$ range and by AHB angle situated between $90^{\circ}$ and $150^{\circ}$. Quantum chemical calculation on isolated dimers completes these structural information as illustrated in Figure 31 for several simple systems.

Directional interaction of the $\mathrm{AH}$ donor toward a nonbonding electron pair of $\mathrm{B}$ (the first rule of Legon and Millen (1987)) is apparent for the $(\mathrm{HF})_{2}\left(\mathrm{OH}_{2}\right)_{2}$ and $\left(\mathrm{NH}_{3}\right)_{2}$ complexes, the AHB angle of $\sim 170^{\circ}$ corresponds probably to the minimum repulsion between the hydrogen atoms not involved in the hydrogen bond. The second rule of Legon and Millen (1987) (point (ii) above) is clearly illustrated by the structure of the $\mathrm{C}_{2} \mathrm{H}_{4} \cdots \mathrm{HF}$ complex.

For more complex molecules however the prediction of the probable structure of hydrogen bonded complexes is not straightforward. In fact, as established by theory (Chan et al.,

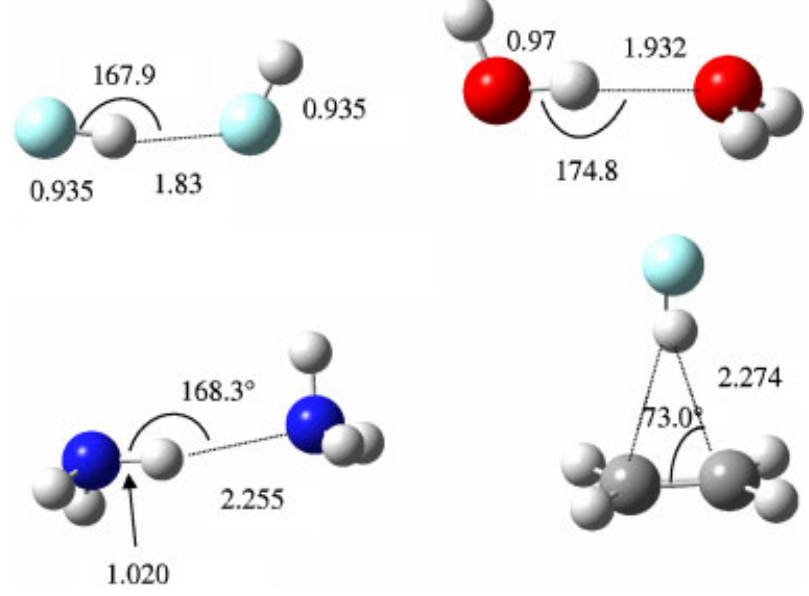

FIGURE 31. Optimized (B3LYP/6-311+ G(d,p) level) geometries of selected hydrogen bonded neutral dimers $(\mathrm{HF})_{2},\left(\mathrm{OH}_{2}\right)_{2},\left(\mathrm{NH}_{3}\right)_{2}$, $\mathrm{C}_{2} \mathrm{H}_{4} \cdots \mathrm{HF}$. [Color figure can be viewed in the online issue, which is available at www.interscience.wiley.com.] 


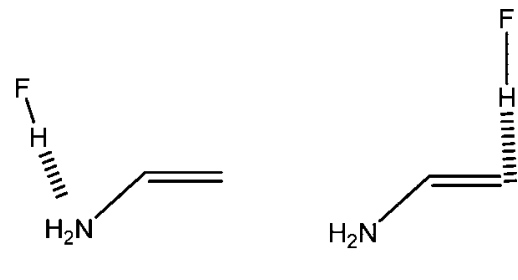

$a$

$b$

SCHEME 15.

2005), hydrogen bond formation is essentially influenced by electrostatic interactions: the preferred site for hydrogen bonding corresponds to the more localized negative charge. It does not necessarily correspond to the most basic site. For example FH leads to a preferred complex with vinylamine at the nitrogen atom rather than at the carbon $\mathrm{C}(2)$ which is the preferred site of protonation. The energy difference calculated between the two complexes $\boldsymbol{a}$ and $\boldsymbol{b}$ is equal to $12 \mathrm{~kJ} \mathrm{~mol}^{-1}$ (Scheme 15 ).

Other examples of molecules for which hydrogen bond and protonation occur at different sites are given in Scheme 16. 1HAzirine and azete form hydrogen bond complexes (indicated by a dotted arrow) preferentially on the nitrogen atom rather than on their most basic site (indicated by a large arrow). In pyrrole, the electron pair of the nitrogen atom is too largely delocalized to act as hydrogen bond acceptor, in that regard, position $\mathrm{C}(3)$ is favored but as already mentioned the most basic site is carbon $\mathrm{C}(2)$. Finally furan gives more favorable hydrogen bonding at oxygen and position $\mathrm{C}(3)$ whereas the preferential protonation site is again $\mathrm{C}(2)$.

In a similar vein, the most stable water/hydrogen cyanide cluster is $\mathrm{H}_{2} \mathrm{O} \cdots \mathrm{HCN}$, not $\mathrm{HCN} \cdots \mathrm{H}_{2} \mathrm{O}$ which lies $6 \mathrm{~kJ} \mathrm{~mol}^{-1}$ above, although the proton affinity of $\mathrm{HCN}$ is higher than that of $\mathrm{H}_{2} \mathrm{O}$ by ca. $20 \mathrm{~kJ} \mathrm{~mol}^{-1}$ (Malaspina et al., 2006).

The energetic aspect of the hydrogen bonded gas-phase neutral clusters has been also explored both experimentally and theoretically. Several experimental enthalpies of dissociation of symmetrical neutral dimers $\mathrm{A}-\mathrm{H} \cdots \mathrm{A}$ are listed in Table 8 (Curtiss \& Blander, 1988). It clearly appears that, in the majority of cases, the dissociation enthalpy falls in the limited range of values $15-20 \mathrm{~kJ} \mathrm{~mol}^{-1}$. The maximum value, $63 \mathrm{~kJ} \mathrm{~mol}^{-1}$, corresponds to the particular case of the formic acid dimer which contains two hydrogen bonds reinforced by a favorable, cooperative, dipole-dipole interaction. Another important point to note is that the dimers of acetonitrile, pyridine and acetone exhibits similar binding energies whereas the hydrogen bonds involve participation of a $\mathrm{C}-\mathrm{H}$ donor moiety. For these three molecules however, the carbon of the $\mathrm{C}-\mathrm{H}$ group is substituted by an electron attractor group which gives an acidic character of the $\mathrm{H}$ atom involved in the hydrogen bond. Accordingly, when the $\mathrm{C}-\mathrm{H}$ donor group is unsubstituted, such as in acetylene, ethylene or ethane the binding energy with, for example water, is limited to 7, 1.5 , and $0.3 \mathrm{~kJ} \mathrm{~mol}^{-1}$, respectively (Hartmann, Westmore, \& Radom, 2001).

b. Protonated hydrogen bonded dimers. Information concerning isolated proton bonded complexes $\mathrm{AH}^{+} \mathrm{B}$ and originating from experiment and theory, are also numerous (Jeffrey, 1997; Scheiner, 1997; Mautner, 2005). An important characteristic of hydrogen bonding is that it is considerably reinforced when a net electric charge is involved since electrostatic and polarization interactions are dramatically enhanced. Accordingly, since hydrogen bond energy between two neutral molecules is typically equal to ca. $15-20 \mathrm{~kJ} \mathrm{~mol}^{-1}$ as shown above in Table 8 , this quantity attains value in the range $50-150 \mathrm{~kJ} \mathrm{~mol}^{-1}$ if one partner is an ionic specie. This is established from the experimental determination of intermolecular ionic hydrogen bond energies, that is, the enthalpy of the clustering reaction (c), $\Delta_{\mathrm{c}} H^{0}$ :

$$
\mathrm{AH}^{+}+\mathrm{B} \rightarrow \mathrm{AH}^{+} \cdots \mathrm{B}
$$

obtained, for a large number of couples of bases A and B, during the last thirty years (Davidson, Sunner, \& Kebarle, 1979; Larson \& McMahon, 1982; Mautner, 1984; Mautner \& Sieck, 1985; Speller \& Mautner, 1985) and for which representative examples are reported in Table 9.

Hydrogen bonding properties of $\mathrm{AH}^{+} \mathrm{B}$ complexes and protonation energetics of $\mathrm{A}$ and $\mathrm{B}$ are intuitively suspected to be related. When a protonated species $\mathrm{AH}^{+}$encounters a base $\mathrm{B}$, the attractive electrostatic interaction would lead to an $\mathrm{AH}^{+} \ldots \mathrm{B}$ complex. Moreover, it is possible that a proton transfer occurs inside the complex resulting in another complex $\mathrm{A} \cdots \mathrm{HB}^{+}$. For gas-phase proton transfer reaction occurring without particular constraint, the proton donor and the acceptor base orient themselves to find the geometrical arrangement minimizing the energy. A simple view of the formation of the $\mathrm{AH}^{+} \mathrm{B}$ complexes is that the proton will be closer to the base of the larger proton affinity (Scheme 17). Thus an $\mathrm{AH}^{+} \ldots \mathrm{B}$ complex is formed when the proton affinity of $\mathrm{A}$ is larger than that of $\mathrm{B}$. The hydrogen bond in $\mathrm{AH}^{+} \ldots \mathrm{B}$ is then due to a partial electron donation of the less basic species $\mathrm{B}$ to the proton donor group. This is expected to be more efficient when the base $B$ is as stronger as possible with the constraint that $\mathrm{PA}(\mathrm{B})$ should be lower than $\mathrm{PA}(\mathrm{A})$. Consequently, a strong hydrogen bond should be observed when $\mathrm{A}$ and $\mathrm{B}$ have similar proton affinities.
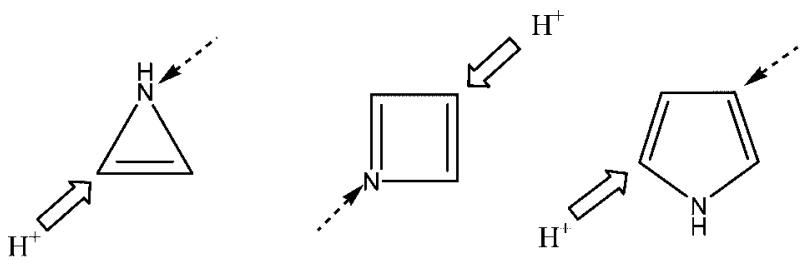

SCHEME 16.

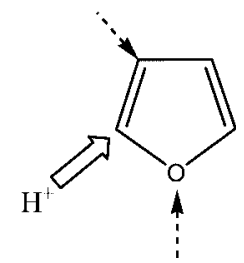


TABLE 8. Experimental ${ }^{\mathrm{a}}$ enthalpies of dissociation of $\mathrm{AH} \cdots \mathrm{A}$ neutral dimers $\left(\mathrm{kJ} \mathrm{mol}^{-1}\right)^{\mathrm{a}}$

\begin{tabular}{cc}
\hline $\mathrm{A}$ & $\Delta_{\mathrm{d}} \mathrm{H}^{\circ}$ \\
\hline $\mathrm{H}_{2} \mathrm{O}$ & 15 \\
$\mathrm{CH}_{3} \mathrm{OH}$ & 15 \\
$\left(\mathrm{CH}_{3}\right)_{3} \mathrm{COH}$ & 19 \\
$\mathrm{HF}$ & 12 \\
$\mathrm{HCl}$ & 10 \\
$\mathrm{NH}_{3}$ & 18 \\
$\mathrm{HCOOH}^{\mathrm{CH}_{3} \mathrm{CN}}$ & 63 \\
pyridine & 21 \\
$\left(\mathrm{CH}_{3}\right)_{2} \mathrm{CO}$ & 21 \\
\hline
\end{tabular}

${ }^{\mathrm{a}}$ Experimental values from Curtiss and Blander (1988).

This is completely confirmed by the experimental results presented in Table 9: the symmetric proton bound dimers $\mathrm{AH}^{+} \ldots$ A present the largest $\Delta_{\mathrm{c}} H^{0}$ values. For example the $\Delta_{\mathrm{c}} H^{0}$ of $\mathrm{AH}^{+} \ldots \mathrm{NH}_{3}$ clusters is equal to $104 \mathrm{~kJ} \mathrm{~mol}^{-1}$ when $\mathrm{A}=\mathrm{NH}_{3}$ and it progressively decreases to 90,86 , and 72 when A becomes methylamine, dimethylamine and trimethylamine, respectively (Mautner, 1984; Table 9). Another point that emerges from the experimental data is that the binding energy of symmetric protonated clusters, $\Delta_{\mathrm{c}} H_{\mathrm{sym}}^{0}$, increases when the proton affinity of the base A decreases. This may be readily explained by the fact that when $\mathrm{A}$ is a weak base it has a limited tendency to give electrons to the proton donor $\mathrm{AH}^{+}$and thus the $\mathrm{AH}^{+} \cdots \mathrm{A}$ distance is shortened resulting in an increase of the electrostatic attraction.

The observation that unsymetrical complexes are less stabilized than the symmetrical systems has been expressed in the form of a relationship involving the difference in proton affinities of $\mathrm{A}$ and $\mathrm{B}$ :

$$
\Delta_{\mathrm{c}} H^{0}=\Delta_{\mathrm{c}} H_{\mathrm{sym}}^{0}-\alpha[\mathrm{PA}(\mathrm{A})-\mathrm{PA}(\mathrm{B})]
$$

where $\Delta_{\mathrm{c}} H_{\mathrm{sym}}^{0}$ is the hydrogen bond energy of the symmetrical protonated dimer $\mathrm{AH}^{+} \ldots \mathrm{A}$ and $\alpha$ a correlation parameter specific of a given series of couples $\mathrm{A}$ and $\mathrm{B}$. For example $\Delta_{\mathrm{c}} H_{\mathrm{sym}}^{0}=$ $129 \pm 8 \mathrm{~kJ} \mathrm{~mol}^{-1}$ and $\alpha=0.46$ for oxygen bases (Larson \& McMahon, 1982) while these two quantities are $97 \pm 3 \mathrm{~kJ} \mathrm{~mol}^{-1}$ and $0.26 \pm 0.03$, respectively, for nitrogen bases, except cyanides (Mautner, 1984). Extension of the correlation of $\Delta_{\mathrm{c}} H^{0}$ with $[\mathrm{PA}(\mathrm{A})-\mathrm{PA}(\mathrm{B})]$ to mixed protonated dimers has been proposed via the use of the general equation:

$$
\Delta_{\mathrm{c}} H^{0}=\Delta_{\mathrm{c}} H_{\mathrm{AB}}^{0}-\alpha[\mathrm{PA}(\mathrm{A})-\mathrm{PA}(\mathrm{B})]
$$

the parameters of which are given in Table 10 for oxygen nitrogen and sulfur bases (Mautner, 1984; Mautner \& Sieck, 1985).

The structures of the complexes, and particularly the length of the ionic hydrogen bond, as revealed by quantum chemical calculations, reflect the above energetic considerations as shown in Figure 32 for simple systems.

Considering a larger amount of data, Jeffrey noticed that strong (ionic) hydrogen bonds are mostly covalent and are characterized by comparable $\mathrm{AH}$ and $\mathrm{HB}$ distances (the "hesitating proton") in the 1.2-1.5 ^ range and by AHB angles close to $180^{\circ}$. Accordingly, Figure 32 shows that the hydrogen bonding atom is situated at the center of the $\mathrm{AB}$ distance for $(\mathrm{HF})_{2} \mathrm{H}^{+}$and $\left(\mathrm{OH}_{2}\right)_{2} \mathrm{H}^{+}$. In the case of $\left(\mathrm{NH}_{3}\right)_{2} \mathrm{H}^{+}$however, the most stable structure (at the B3LYP/6-311+G(d,p) level) is somewhat asymmetric. In fact, the shift of the central hydrogen from one nitrogen to the other is associated with a negligible activation barrier of $1.1 \mathrm{~kJ} \mathrm{~mol}^{-1}$ (in the transition structure the two NH bonds are equal to $1.31 \AA$ ). For these three examples the $\mathrm{AHB}$ angle is very close to $180^{\circ}$. By contrast, the complex formed between formaldehyde and protonated ammonia exhibits an angular structure (Fig. 32): the directional effect of the lone pair situated on the oxygen atom is combined with the repulsion between the remaining three $\mathrm{H}$ of the ammonia and the methylene group.

It has been found rapidly that the correlation of $\Delta_{\mathrm{c}} H^{0}$ with $[\mathrm{PA}(\mathrm{A})-\mathrm{PA}(\mathrm{B})]$ given by Equation (101) was not sufficient to precisely predict the $\Delta_{\mathrm{c}} H^{0}$ value in all situations. Accordingly, Speller and Mautner (1985) show that the experimental $\Delta_{\mathrm{c}} H^{0}$ of clusters involving cyanides are substantially stronger than predicted by Equation (101) with the parameters $\Delta_{\mathrm{c}} H_{\text {sym }}^{0}$ and $\alpha$ of $\mathrm{NH}^{+} \ldots \mathrm{N}$ bonds (see also Table 9). This anomaly was interpreted by the change in the hydrogen bond strength due to the large dipole moment of the cyanides.

In fact, the details of the interaction that occurs during the formation of a hydrogen bond between $\mathrm{AH}^{+}$and $\mathrm{B}$ can be influenced by characteristics of the products $\mathrm{A}$ and $\mathrm{B}$ other than exclusively that related to the Bronsted basicity. As shown above (see Characteristics Related to the Nature of the Basic Site) the preferred site for protonation of a base $\mathrm{A}$ is mainly determined by the stability of the resulting cation $\mathrm{AH}^{+}$. It consequently depends on the ability of the ion $\mathrm{AH}^{+}$to stabilize the positive charge by electrostatic and, often mostly, resonance effects. On the other hand, it as been also mentioned (see Scheme 15 and the corresponding discussion) that the preferred site for hydrogen bonding in neutral systems AHB is primarily determined by local electron densities. This latter characteristic may be also extended to protonated $\mathrm{AHB}^{+}$clusters (Chan et al., 2005). Consequently, in polyfunctional molecules, the two criteria may not be fulfilled by the same functional group and protonation or proton bonding may occur at different sites.

Complexes formed between $\mathrm{NH}_{4}{ }^{+}$and vinylamine or furan, for example, present an ionic hydrogen bond pointing on atoms which are not the preferred sites of protonation (Scheme 18). In the former case one $\mathrm{H}$ of the ammonium ion points toward the 
TABLE 9. Experimental ionic hydrogen bond energies $\Delta_{\mathrm{c}} H$ of $\mathrm{AH}^{+} \ldots \mathrm{B}$ clusters $\left(\mathrm{kJ} \mathrm{mol}^{-1}\right)$

\begin{tabular}{|c|c|c|}
\hline $\mathbf{A}$ & B & $\Delta_{c} H$ \\
\hline $\mathrm{H}_{2} \mathrm{O}$ & $\mathrm{H}_{2} \mathrm{O}$ & $132^{a}$ \\
\hline $\mathrm{CH}_{3} \mathrm{OH}$ & $\mathrm{CH}_{3} \mathrm{OH}$ & $131^{\mathrm{a}}$ \\
\hline$\left(\mathrm{CH}_{3}\right)_{2} \mathrm{O}$ & $\left(\mathrm{CH}_{3}\right)_{2} \mathrm{O}$ & $128^{\mathrm{a}}, 123^{\mathrm{b}}$ \\
\hline $\mathrm{C}_{2} \mathrm{H}_{5} \mathrm{OC}_{2} \mathrm{H}_{5}$ & $\mathrm{C}_{2} \mathrm{H}_{5} \mathrm{OC}_{2} \mathrm{H}_{5}$ & $125^{\mathrm{b}}$ \\
\hline $\mathrm{CH}_{3} \mathrm{CHO}$ & $\mathrm{CH}_{3} \mathrm{CHO}$ & $132^{\mathrm{a}}$ \\
\hline$\left(\mathrm{CH}_{3}\right)_{2} \mathrm{CO}$ & $\left(\mathrm{CH}_{3}\right)_{2} \mathrm{CO}$ & $134^{\mathrm{a}}$ \\
\hline $\mathrm{CH}_{3} \mathrm{COOH}$ & $\mathrm{CH}_{3} \mathrm{COOH}$ & $124^{\mathrm{a}}$ \\
\hline $\mathrm{HCOO} \mathrm{C}_{2} \mathrm{H}_{5}$ & $\mathrm{HCOO} \mathrm{C}_{2} \mathrm{H}_{5}$ & $123^{\mathrm{a}}$ \\
\hline tetrahydrofuran & tetrahydrofuran & $138^{a}, 136^{b}$ \\
\hline $\mathrm{NH}_{3}$ & $\mathrm{NH}_{3}$ & $104^{b}$ \\
\hline $\mathrm{CH}_{3} \mathrm{NH}_{2}$ & $\mathrm{CH}_{3} \mathrm{NH}_{2}$ & $91^{\mathrm{b}}$ \\
\hline$\left(\mathrm{CH}_{3}\right)_{3} \mathrm{~N}$ & $\left(\mathrm{CH}_{3}\right)_{3} \mathrm{~N}$ & $95^{\mathrm{b}}$ \\
\hline pyridine & pyridine & $103^{\mathrm{b}}$ \\
\hline $\mathrm{CH}_{3} \mathrm{CN}$ & $\mathrm{CH}_{3} \mathrm{CN}$ & $126^{b}$ \\
\hline $\mathrm{H}_{2} \mathrm{~S}$ & $\mathrm{H}_{2} \mathrm{~S}$ & $55-65^{d}$ \\
\hline$\left(\mathrm{CH}_{3}\right)_{2} \mathrm{~S}$ & $\left(\mathrm{CH}_{3}\right)_{2} \mathrm{~S}$ & $110^{\mathrm{d}}$ \\
\hline $\mathrm{H}_{2} \mathrm{O}$ & $\mathrm{CH}_{2} \mathrm{O}$ & $135^{\mathrm{a}}$ \\
\hline $\mathrm{CH}_{3} \mathrm{OH}$ & $\mathrm{CH}_{3} \mathrm{CHO}$ & $135^{\mathrm{a}}$ \\
\hline $\mathrm{CH}_{3} \mathrm{OH}$ & $\mathrm{H}_{2} \mathrm{O}$ & $114^{\mathrm{b}}$ \\
\hline $\mathrm{CH}_{3} \mathrm{CHO}$ & $\mathrm{C}_{2} \mathrm{H}_{5} \mathrm{OH}$ & $135^{\mathrm{a}}$ \\
\hline $\mathrm{CH}_{3} \mathrm{CHO}$ & $\mathrm{CH}_{3} \mathrm{COOH}$ & $134^{a}$ \\
\hline $\mathrm{CH}_{3} \mathrm{CHO}$ & $\mathrm{H}_{2} \mathrm{O}$ & $105^{\mathrm{b}}$ \\
\hline $\mathrm{C}_{2} \mathrm{H}_{5} \mathrm{OH}$ & $\mathrm{CH}_{3} \mathrm{COOH}$ & $128^{\mathrm{a}}$ \\
\hline $\mathrm{CH}_{3} \mathrm{COOH}$ & $\left(\mathrm{CH}_{3}\right)_{2} \mathrm{O}$ & $13^{\mathrm{a}}$ \\
\hline $\mathrm{CH}_{3} \mathrm{COOH}$ & $\mathrm{H}_{2} \mathrm{O}$ & $84^{b}$ \\
\hline$\left(\mathrm{CH}_{3}\right)_{2} \mathrm{O}$ & $\mathrm{HCOO} \mathrm{C}_{2} \mathrm{H}_{5}$ & $131^{\mathrm{a}}$ \\
\hline$\left(\mathrm{CH}_{3}\right)_{2} \mathrm{O}$ & $\mathrm{H}_{2} \mathrm{O}$ & $100^{\mathrm{b}}$ \\
\hline$\left(\mathrm{CH}_{3}\right)_{2} \mathrm{CO}$ & $\mathrm{CH}_{3} \mathrm{COOCH}_{3}$ & $134^{\mathrm{a}}$ \\
\hline$\left(\mathrm{CH}_{3}\right)_{2} \mathrm{CO}$ & $\mathrm{H}_{2} \mathrm{O}$ & $88^{\mathrm{b}}$ \\
\hline$\left(\mathrm{CH}_{3}\right)_{2} \mathrm{CO}$ & tetrahydrofuran & $142^{\mathrm{a}}$ \\
\hline $\mathrm{NH}_{3}$ & $\mathrm{H}_{2} \mathrm{O}$ & $86^{\mathrm{b}}$ \\
\hline $\mathrm{NH}_{3}$ & $\mathrm{HCN}$ & $86^{\circ}$ \\
\hline $\mathrm{NH}_{3}$ & $\mathrm{H}_{2} \mathrm{~S}$ & $48^{d}$ \\
\hline $\mathrm{NH}_{3}$ & $\mathrm{CH}_{3} \mathrm{CN}$ & $115^{\mathrm{c}}$ \\
\hline $\mathrm{CH}_{3} \mathrm{NH}_{2}$ & $\mathrm{H}_{2} \mathrm{O}$ & $77^{\mathrm{b}}$ \\
\hline $\mathrm{CH}_{3} \mathrm{NH}_{2}$ & $\mathrm{CH}_{3} \mathrm{~F}$ & $49^{b}$ \\
\hline $\mathrm{CH}_{3} \mathrm{NH}_{2}$ & $\mathrm{NH}_{3}$ & $90^{b}$ \\
\hline $\mathrm{CH}_{3} \mathrm{NH}_{2}$ & $\mathrm{HCN}$ & $87^{c}$ \\
\hline $\mathrm{CH}_{3} \mathrm{NH}_{2}$ & $\mathrm{CH}_{3} \mathrm{CN}$ & $103^{b}, 110^{c}$ \\
\hline $\mathrm{CH}_{3} \mathrm{NH}_{2}$ & $\mathrm{H}_{2} \mathrm{~S}$ & $45^{\mathrm{d}}$ \\
\hline $\mathrm{CH}_{3} \mathrm{NH}_{2}$ & $\mathrm{CH}_{3} \mathrm{SH}$ & $56^{\mathrm{d}}$ \\
\hline$\left(\mathrm{CH}_{3}\right)_{2} \mathrm{NH}$ & $\mathrm{H}_{2} \mathrm{O}$ & $68^{b}$ \\
\hline$\left(\mathrm{CH}_{3}\right)_{2} \mathrm{NH}$ & $\mathrm{NH}_{3}$ & $86^{\mathrm{b}}$ \\
\hline$\left(\mathrm{CH}_{3}\right)_{2} \mathrm{NH}$ & $\mathrm{CH}_{3} \mathrm{NH}_{2}$ & $94^{\mathrm{b}}$ \\
\hline$\left(\mathrm{CH}_{3}\right)_{3} \mathrm{~N}$ & $\mathrm{H}_{2} \mathrm{O}$ & $64^{b}$ \\
\hline$\left(\mathrm{CH}_{3}\right)_{3} \mathrm{~N}$ & $\mathrm{HCN}$ & $70^{\mathrm{c}}$ \\
\hline$\left(\mathrm{CH}_{3}\right)_{3} \mathrm{~N}$ & $\left(\mathrm{CH}_{3}\right)_{2} \mathrm{NH}$ & $86^{\mathrm{b}}$ \\
\hline$\left(\mathrm{CH}_{3}\right)_{3} \mathrm{~N}$ & $\mathrm{CH}_{3} \mathrm{NH}_{2}$ & $94^{\mathrm{b}}$ \\
\hline Pyridine & $\mathrm{NH}_{3}$ & $72^{b}$ \\
\hline Pyridine & $\mathrm{H}_{2} \mathrm{O}$ & $67^{b}$ \\
\hline pyridine & $\left(\mathrm{n}-\mathrm{C}_{3} \mathrm{H}_{7}\right)_{2} \mathrm{O}$ & $98^{b}$ \\
\hline $\mathrm{CH}_{3} \mathrm{CN}$ & $\mathrm{H}_{2} \mathrm{O}$ & $95^{\mathrm{c}}$ \\
\hline $\mathrm{CH}_{3} \mathrm{CN}$ & $\mathrm{HCN}$ & $92^{\mathrm{c}}$ \\
\hline $\mathrm{H}_{2} \mathrm{~S}$ & $\mathrm{H}_{2} \mathrm{O}$ & $71-88^{d}$ \\
\hline $\mathrm{CH}_{3} \mathrm{SH}$ & $\mathrm{H}_{2} \mathrm{O}$ & $65^{\mathrm{d}}$ \\
\hline$\left(\mathrm{CH}_{3}\right)_{2} \mathrm{~S}$ & $\mathrm{H}_{2} \mathrm{O}$ & $55^{\mathrm{d}}$ \\
\hline
\end{tabular}




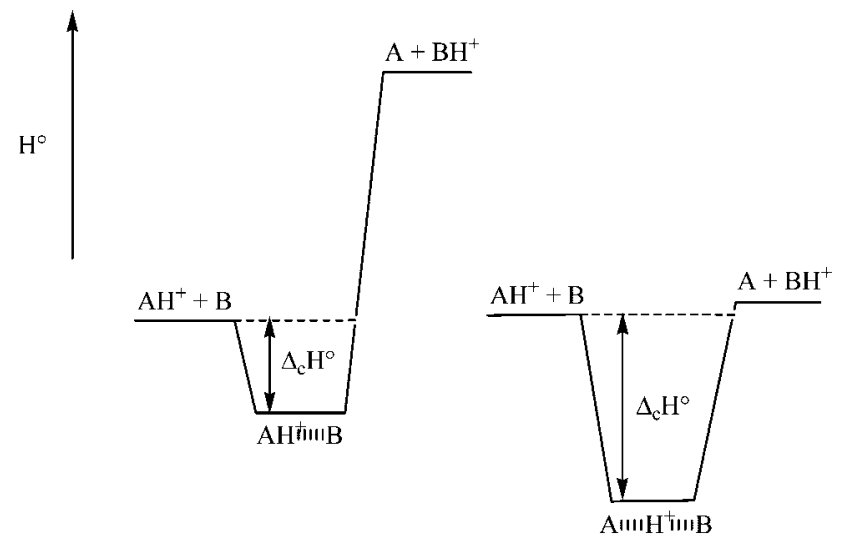

(a) $\quad \mathrm{PA}(\mathrm{A})>\mathrm{PA}(\mathrm{B}) \quad$ (b) $\quad \mathrm{PA}(\mathrm{A}) \sim \mathrm{PA}$ (B)

SCHEME 17.

nitrogen of the vinylamine while the complex between furan and $\mathrm{NH}_{4}{ }^{+}$involves two hydrogen bonds with the $\pi$-electron system at $\mathrm{C}(3)$ and $\mathrm{O}$.

Similarly, to return to the cyanide case, the structure of the adducts of the type $\mathrm{RCNH}^{+}+\mathrm{O}(\mathrm{H}) \mathrm{R}^{\prime}$ does not conform to what may be expected from the proton affinities. Figure 34 shows the example of $\mathrm{R}=\mathrm{R}^{\prime}=\mathrm{CH}_{3}$, the proton is calculated to be closer to the oxygen atom of the methanol molecule $(d=1.117 \AA)$ than to the nitrogen of methyl cyanide $(d=1.365 \AA)$ whereas the proton affinity of methanol, $\mathrm{PA}\left(\mathrm{CH}_{3} \mathrm{OH}\right)=754 \mathrm{~kJ} \mathrm{~mol}^{-1}$, is lower than that of methyl cyanide, $\mathrm{PA}\left(\mathrm{CH}_{3} \mathrm{CN}\right)=779 \mathrm{~kJ} \mathrm{~mol}^{-1}$ (Mayer, 1999; Fridgen, Keller, \& McMahon, 2001).

It is generally predicted by theory that there is no particular barrier for the proton transfer reaction if no geometrical constraint is imposed between the two basic sites A and B. The potential energy surface presents only one minimum corresponding to the optimum, $\mathrm{AH}^{+} \cdots \mathrm{B}$ or $\mathrm{A} \cdots \mathrm{HB}^{+}$, arrangement as described in Scheme 17. Formally, the proton transfer potential energy surface may be decomposed in two potential wells corresponding to the $\mathrm{A}-\mathrm{H}$ and $\mathrm{H}-\mathrm{B}$ elongations. When there is no constraint imposed between the two basic sites $\mathrm{A}$ and $\mathrm{B}$, the optimum distance separating the two minima is reduced to its minimum value which generally corresponds to the absence of a central barrier (or to a barrier lower than the zero point vibrational energy). At the opposite, when the distance between the two minima is sufficiently large, the intercept of the two curves occurs at high potential energy and an energy barrier may be present. This behavior is expected when steric hindrance imposes a A $\cdots$ B distance larger than the optimum value (Scheiner, 1985).

c. Protonation of solvated species. Protonation thermochemistry of solvated molecules has been the subject of an important number of studies (Ng, Baer, \& Powis, 1993; Stace, 1995; Mautner, 2005) which may be considered out of the scope of the present review. However interesting observation may be extracted from the behavior of molecules or ions in chemically inactive medium such as noble gas environment. A recent investigation on $\mathrm{NH}_{3}(\mathrm{Ar})_{n}$ and $\mathrm{NH}_{4}{ }^{+}(\mathrm{Ar})_{n}$ clusters shows that, due to the better stabilization energies of the latter, the proton affinity of ammonia increases with the number of ligands (Szymczak et al., 2006). The increase in proton affinity is ca. $10 \mathrm{~kJ} \mathrm{~mol}^{-1}$ when adding the first molecule of Argon and slowly decreases with increasing $n$. This shows indeed again that the energetic of the protonation is sensitive to the stabilization of the protonated form by charge/polarizable interactions.

\section{Intramolecular Hydrogen Bonding}

The importance of intramolecular hydrogen bonds in the structures and functions of biological macromolecules is now evidence. The first proposal that helical or sheet structures of proteins and desoxynucleic acids are dictated by hydrogen bonds is presently widely extrapolated to every aspect of biochemical and biological processes (Jeffrey \& Saenger, 1994). When we are dealing with isolated molecules or ions, not subjected to interactions with solvent molecules, intramolecular hydrogen bonds may freely intervene and contribute to the intrinsic stability of the considered species. Consequently, their role in the gas-phase basicity of polyfunctional molecules is essential.

a. Intramolecular hydrogen bonds in neutral molecules. Intramolecular hydrogen bonding is expected if the considered molecule contains both the donor group $\mathrm{X}-\mathrm{H}$ and the acceptor part $\mathrm{Y}$ in such a way that the $\mathrm{X}-\mathrm{H}$. . Y system may be formed without insurmountable constraint. Consequence of intramolecular hydrogen bond formation is a gain in stability which will favor particular conformations of the (necessarily polyfunctional) molecule.

TABLE 10. Correlation coefficients $\Delta_{\mathrm{c}} H_{\mathrm{AB}}^{0}\left(\mathrm{~kJ} \mathrm{~mol}^{-1}\right)$ and $\alpha$ for $\mathrm{AH}^{+} \cdots \mathrm{B}$ clusters

\begin{tabular}{cccc}
\hline $\mathrm{A}$ & $\mathrm{B}$ & $\Delta_{\mathrm{c}} \mathrm{H}_{\mathrm{AB}}{ }^{\circ}$ & $\alpha$ \\
\hline $\mathrm{O}$ & $\mathrm{O}$ & $129 \pm 8^{\mathrm{a}}$ & 0.46 \\
$\mathrm{~N}$ & $\mathrm{O}$ & $126 \pm 6^{\mathrm{b}}$ & $0.26 \pm 0.03$ \\
$\mathrm{~N}$ & $\mathrm{~N}$ & $97 \pm 3^{\mathrm{b}}$ & $0.25 \pm 0.05$ \\
$\mathrm{~N}$ & $\mathrm{~S}$ & $94 \pm 3^{\mathrm{b}}$ & $0.26 \pm 0.03$ \\
$\mathrm{~S}$ & $\mathrm{O}$ & $78 \pm 1^{\mathrm{c}}$ & $0.16 \pm 0.01$ \\
\hline
\end{tabular}

${ }^{\mathrm{a}}$ Larson and McMahon (1982).

${ }^{\mathrm{b}}$ Mautner (1984).

${ }^{\mathrm{c}}$ Mautner and Sieck (1985). 

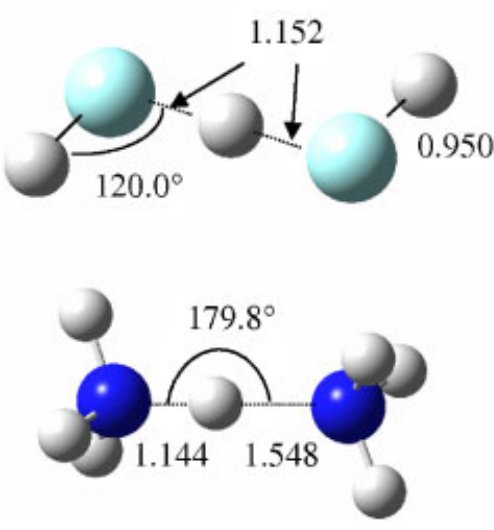

1.197

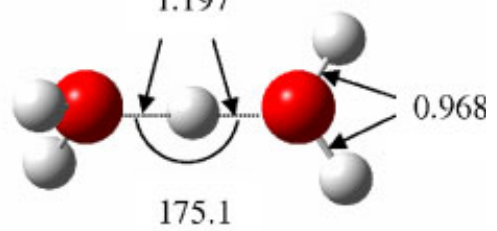

1.659

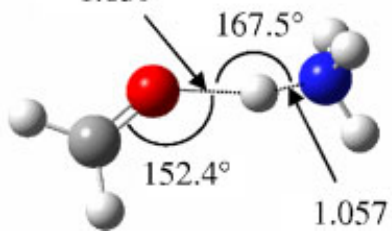

FIGURE 32. Optimized (B3LYP/6-311+ G(d,p) level) geometries of selected protonated dimers $(\mathrm{HF})_{2} \mathrm{H}^{+},\left(\mathrm{OH}_{2}\right)_{2} \mathrm{H}^{+},\left(\mathrm{NH}_{3}\right)_{2} \mathrm{H}^{+}, \mathrm{CH}_{2} \mathrm{O} \cdots \mathrm{HNH}_{3}+$. [Color figure can be viewed in the online issue, which is available at www.interscience.wiley.com.]

A direct estimation of intramolecular hydrogen bond energy is however not an easy task because of the difficulties to separate its own characteristics from the other bonding properties. Approaches based on the comparison between conformers and isomers of simplified homologues (Deshmukh et al., 2006), where the hydrogen bond is either retained or non-existent, are invariably limited by the fact that (at least) some dipole orientations are different in the compared systems. It remains however a simple mean to have an idea of the gain in stability provided by the internal hydrogen bonding. $\alpha, \omega$-Aminoalcohols may serve to illustrate the role of intramolecular hydrogen bond in the structure and the stability of neutral bifunctional molecules. For 1,2-aminoethanol, 1, 1,3-aminopropanol, 2, and 1,4-aminobutanol, $\mathbf{3}$, the most stable conformations are the pseudo-chair $\mathbf{1 a}-\mathbf{3 a}$ depicted in Figure 34 (Bouchoux et al., 2002). These conformations are characterized by the existence of an internal $\mathrm{OH}$. . N hydrogen bond, evidenced by a dotted line in Figure 34. The calculated $\mathrm{OH} \cdots \mathrm{N}$ distance is decreasing from 2.175 to $1.848 \AA$ when passing from $\mathbf{1 a}$ to $\mathbf{3 a}$ (Table 11). Simultaneously, the OHN angle tends toward the $160-170^{\circ}$ $\mathrm{AH} \cdots \mathrm{B}$ angle observed for neutral alcohol-amines dimers (see for example Fig. 31). It is expected that the $\mathrm{OH}$. . . N shortening and the $\mathrm{OHN}$ angle opening should be associated with an increase of the hydrogen bond energy. As underlined above, the energy difference between two conformers may be taken as a rough estimate of the intramolecular hydrogen bond energy. This argument may be used here by considering the energy difference $\Delta E$ between the cyclized conformations $\mathbf{1} \boldsymbol{a}-\mathbf{3 a}$ and the fully unfolded structures $\boldsymbol{1} \boldsymbol{b}-\mathbf{3 b}$ (Table 11). In fact, this energy
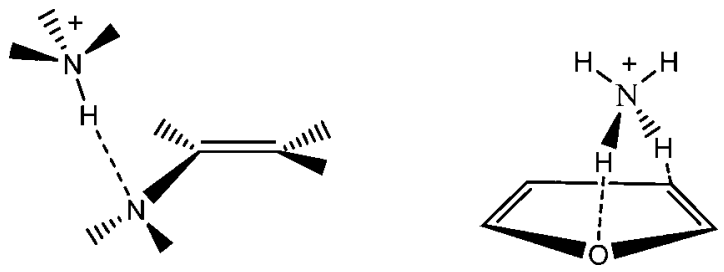

SCHEME 18. difference reflects the balance between the stabilization afforded by the internal hydrogen bond and the destabilization due to the ring strain. As shown in Table 11, the three conformations $\mathbf{1 a}-\mathbf{3 a}$ are increasingly more stable than the fully unfolded structures $\mathbf{1 b}-\mathbf{3 b}$, this result is in line with the decreasing ring strain energy expected when passing from 1 to 3 .

Estimation of the energy of intramolecular hydrogen bond is perhaps more difficult when the counterparts $\mathrm{AH}$ and $\mathrm{B}$ participate to a $\pi$-electron system. The concept of resonance assisted hydrogen bonding (Jeffrey, 1997; Gilli \& Gilli, 2000; Sobczyk, Grabowski, \& Krygowski, 2005), even still a matter of debate (Alkorta et al., 2005), corresponds to situation where the hydrogen bonding may be viewed as an extension of a $\pi$-electron conjugation. This is for example the case for the keto-enol forms of 1,3-dicarbonyls $\boldsymbol{c}$ and 3-amino-2-carbonyl derivatives $\boldsymbol{d}$ (Scheme 19).

A comparison between energies of conformers and di- and trimers allows to suggest that the intramolecular hydrogen bond participate to ca. $20-30 \mathrm{~kJ} \mathrm{~mol}^{-1}$ in the stabilization of the 3aminopropenal and 3-aminopropenthial molecules (structure $\boldsymbol{d}$ in Scheme 19; Jablonsky, Kaszmarek, \& Sadlej, 2006). Such intramolecular hydrogen bond, combined with the energy gain provided by the $\pi$-electron resonance, may assume an excellent

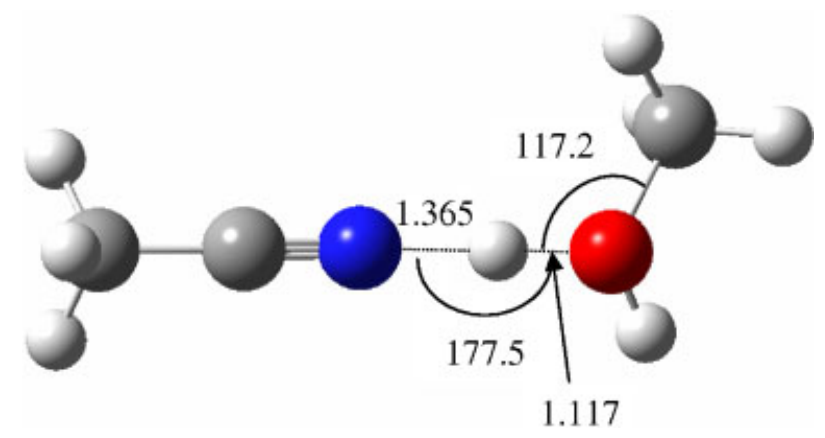

FIGURE 33. Optimized (B3LYP/6-311+ G(d,p) level) geometry of $\mathrm{CH}_{3} \mathrm{CN} \cdots \mathrm{H}^{+} \mathrm{OHCH}_{3}$. [Color figure can be viewed in the online issue, which is available at www.interscience.wiley.com.] 


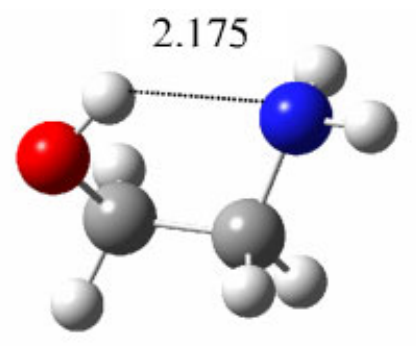

$1 a$

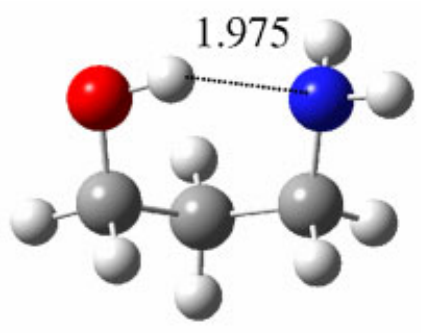

$2 a$

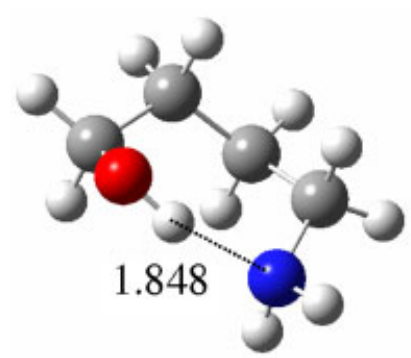

$3 a$

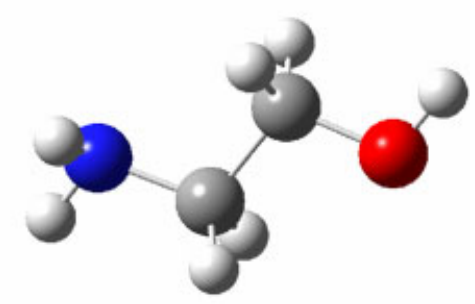

$1 b$

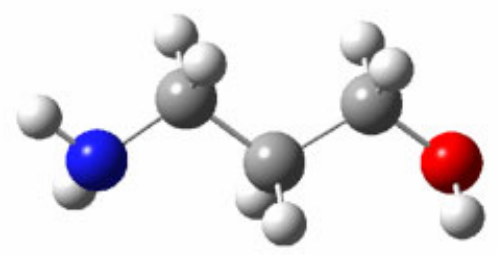

$2 b$

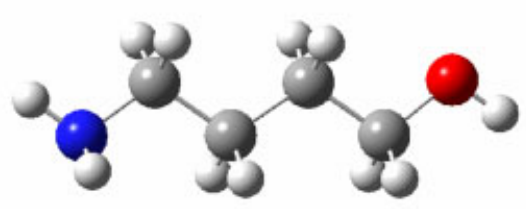

FIGURE 34. B3LYP/6-31G(d) optimized geometries of neutral 1,2-aminoethanol, 1, 1,3-aminopropanol, 2, and 1,4-aminobutanol, 3. [Color figure can be viewed in the online issue, which is available at www.interscience.wiley.com.]

stability to keto-enol or keto-imine molecules in the gasphase, but not necessarily in solution. It appears for example that 1,4-hexanedione is, to more than $99 \%$, in its enol form (4-ol3-penten-2-one) in the gas-phase while the diketo tautomer is major in the tautomeric equilibrium in water solution. b. Intramolecular hydrogen bonds in protonated systems. As mentioned above during the examination of bimolecular systems, the hydrogen bond energies are considerably enhanced when the hydrogen donor is a protonated species. A stronger stabilization is consequently expected during the formation of intramolecular

TABLE 11. Geometrical and energetic characteristics of $\alpha, \omega$-aminoalcohols and their protonated forms ${ }^{\mathrm{a}}$

\begin{tabular}{cccccccc}
\hline $\mathrm{M}$ & $\mathrm{OH} . . . \mathrm{N}(\AA)$ & $\mathrm{OHN}$ angle & $\Delta \mathrm{E}^{\mathrm{b}}$ & $\mathrm{MH}^{+}$ & $\mathrm{O} \ldots \mathrm{HN}(\AA)$ & $\mathrm{OHN}$ angle & $\Delta \mathrm{E}^{\mathrm{b}}$ \\
\hline $\boldsymbol{I}$ & 2.175 & 117.9 & 18 & $\boldsymbol{I H}^{+}$ & 1.981 & 113.7 & 39 \\
$\mathbf{2}$ & 1.975 & 141.2 & 23 & $\mathbf{2 H}^{+}$ & 1.739 & 139.6 & 60 \\
$\mathbf{3}$ & 1.848 & 159.5 & 27 & $\mathbf{3} \boldsymbol{H}^{+}$ & 1.623 & 159.9 & 67 \\
\hline
\end{tabular}

${ }^{\mathrm{a} B} 3 \mathrm{LYP} / 6-31 \mathrm{G}(\mathrm{d})$ optimized geometries.

${ }^{\mathrm{b}} \Delta E$ is the energy difference between the cyclized and linear forms of the system (see Figs. 34 and 35). 


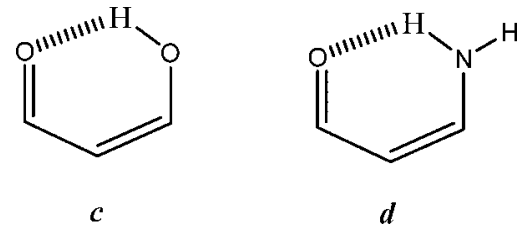

SCHEME 19.

hydrogen bonds in the protonated molecule $\mathrm{MH}^{+}$than in the neutral $\mathrm{M}$. The first consequence is an increase of the proton affinity of the corresponding molecule. This explains why floppy polyfunctional molecules present higher proton affinities than their monofunctional counterparts. Simultaneously, the formation of this strong intramolecular hydrogen bond leads to higher rotational barriers in $\mathrm{MH}^{+}$than in $\mathrm{M}$. The second consequence is that the entropy of the protonated structure becomes lower than that of the neutral base (the protonation entropy $\Delta_{p} S^{0}(\mathrm{M})$ is negative): an entropy loss has occurred during the protonation process.

It has been recognized since the 1970s that the formation of an internal hydrogen bond during protonation of a polydentate base has a considerable impact on the corresponding thermochemistry (Aue, Webb, \& Bowers, 1973). It may be illustrated here by considering again the series of $\alpha, \omega$-aminoalcohols $\mathbf{1 - 3}$. Proton affinities and protonation entropies of these molecules have been determined from measurement of proton transfer equilibrium constant at variable temperature (Mautner et al., 1980). It was found that the proton affinities of $\mathbf{1 - 3}$ are situated between 930 and $980 \mathrm{~kJ} \mathrm{~mol}^{-1}$, that is, significantly higher than that of related primary amines. Accordingly, the proton affinities of propyl to pentylamine are situated in the very narrow range $918-925 \mathrm{~kJ} \mathrm{~mol}^{-1}$. Moreover protonation is accompanied by an important entropy loss particularly for $\mathbf{2}$ and $\mathbf{3}$. These observations were interpreted by the formation of a strong internal hydrogen bond in the protonated form, of increasing strength from 1 to 3 . A quantification of these effects has been done by means of molecular orbital calculations (Bouchoux et al., 2002). Theory predicts that the most stable conformations of the protonated molecules are the cyclized forms $\boldsymbol{I H}^{+}-\mathbf{3} \boldsymbol{H}^{+}$ presented in Figure 35. The three conformers present indeed an internal hydrogen bond between one hydrogen of the protonated amino group and the oxygen of the hydroxyl group. As observed previously for the neutral molecule (Fig. 34) the hydrogen bond length decreases when the size of the molecule increase because the attractive forces of the hydrogen bond are less and less balanced by the ring strain constraints. The global stabilization of the protonated forms thus increases from $\mathbf{H H}^{+}$to $\mathbf{3} \boldsymbol{H}^{+}$. Another indication is the energy difference $\Delta E_{\mathrm{H}}$ calculated between the cyclized conformers $\mathbf{1} \boldsymbol{H}^{+}-\mathbf{3} \boldsymbol{H}^{+}$and their corresponding linear forms. As indicated in Table $11, \Delta E_{\mathrm{H}}$ is (i) increasing with the size of the molecule, and most importantly (ii) higher than $\Delta E$, the equivalent quantity associated to the neutral molecules. This latter point is not surprising but it may be completed by the observation that the maximum value expected for $\Delta E_{\mathrm{H}}$ is the stabilization energy of an equivalent bimolecular system. This latter may be estimated, using Equation (101), to ca. $90 \mathrm{~kJ} \mathrm{~mol}^{-1}$, a value which looks likely for a limiting value of $\Delta E_{\mathrm{H}}$ in Table 11. The net result of the inequality $\Delta E_{\mathrm{H}}>\Delta E$ is that the proton affinity of the molecules increases when passing from 1 to 3 . Theoretical calculations predict $\operatorname{PA}(2)-\operatorname{PA}(\boldsymbol{I})=28 \mathrm{~kJ} \mathrm{~mol}^{-1}$ and $\mathrm{PA}(3)-\mathrm{PA}(2)=14 \mathrm{~kJ} \mathrm{~mol}^{-1}$ while experiment gives slightly higher differences of 34 and $20 \mathrm{~kJ} \mathrm{~mol}^{-1}$, respectively.

Confirmation of the better stabilization of the protonated form with respect to the neutral base is given by the measurement of protonation entropy. Negative protonation entropies of -3 , -44 , and $-65 \mathrm{~J} \mathrm{~mol}^{-1} \mathrm{~K}^{-1}$ were determined for $1-3$ (Mautner et al., 1980). Calculations of the absolute entropy of $\mathbf{1 - 3}$ and their protonated forms reveal also a decrease in entropy after protonation, for reasons that remains to be established, the absolute values however are less than that experimentally determined (Bouchoux et al., 2002).

Examples of polyfunctional molecules supporting intramolecular hydrogen bonds will constitute the major part of the review: $\alpha, \omega$-disubstituted aliphatics, aromatics, aminoacids and derivatives. . . Moreover, it will be seen that when the molecules become more complex, the number of intramolecular hydrogen bond possibilities obviously increases and new phenomenon such as cooperative $\mathrm{H}$ bonds or more complex chemistry involving covalent bond breaking and bond forming may appear.

\section{Covalent Bonding and Protonation}

Direct structural effect of the protonation on covalent bonding has been described for simple systems in Protonation on $\pi$ Electron Systems and will not be repeated here. The consequences of these structural changes, particularly the $\mathrm{C}-\mathrm{XH}$ bond elongation, are more or less complex $\mathrm{C}-\mathrm{X}$ bond dissociations as it

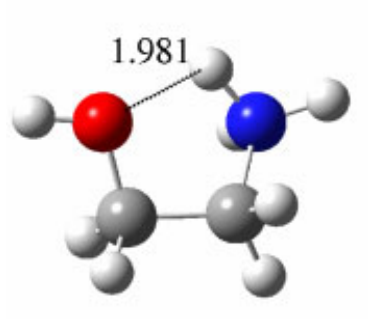

$1 H^{+}$

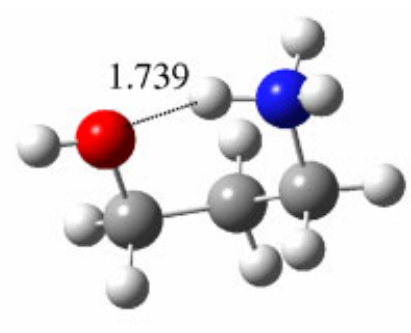

$2 \mathrm{H}^{+}$

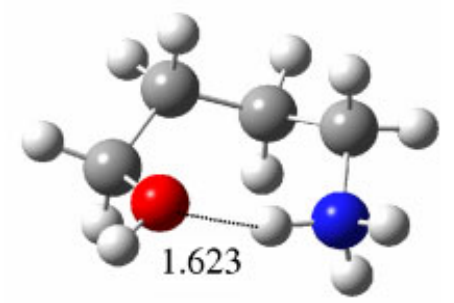

$3 \mathrm{H}^{+}$

FIGURE 35. B3LYP/6-31G(d) optimized geometries of protonated 1,2-aminoethanol, 1, 1,3-aminopropanol, 2, and 1,4-aminobutanol, 3. [Color figure can be viewed in the online issue, which is available at www.interscience.wiley.com.] 
will be briefly recalled below. As described in Background, the experimental determination of the gas-phase protonation thermochemistry involves proton transfer reactions and consequently the intermediacy of hydrogen bonded association adducts. The stabilization energy of such complexes, up to $130 \mathrm{~kJ} \mathrm{~mol}^{-1}$ (Table 9), allows chemistry to occur and, in a number of cases, may lead to products different to what may be expected from the initial molecular structure. Moreover, when a polyfunctional molecule is involved, these bimolecular interactions may become intramolecular and open new reaction routes. The present section presents a survey of the bimolecular and intramolecular processes, induced by the protonation, that lead to significant structural changes (i.e., dissociation, tautomerization, ring opening/ring forming, internal nucleophilic substitution).

\section{Bond Dissociation Induced by the Protonation}

When an electronegative center $\mathrm{X}$ is protonated, the weakening of the $\mathrm{C}-\mathrm{XH}^{+}$bond may lead to a heterolytic cleavage provided the formed cation is a stable species. This simple dissociation process is a well known reaction explaining a large part of the fragmentations from simple protonated molecules such as halogen derivatives, alcohols, ethers, amines, carboxylic acids, esters, and amides to more complex biochemical polymers such as peptides, polysaccharides or nucleic acids whatever the ionization method is (chemical ionization, electrospray, atmospheric pressure chemical ionization, matrix assisted laser desorption ionization...) and whatever the excitation mode is (internal energy of the ion provided directly by the ionization process or after collisional activation or photon absorbtion...). The reader is thus invited to consult the reviews or books related to these mass spectrometric techniques (Harrison, 1992; Splitter \& Turecek, 1994; Gross \& Caprioli, 2003; Gross \& Caprioli, 2005; Paizs \& Suhai, 2005).

Only a reminder of the essential features of $\mathrm{C}-\mathrm{XH}^{+}$bond dissociations is given here. The, direct, simple $\mathrm{C}-\mathrm{XH}^{+}$ dissociation is observed mainly when the formed carbocation is a stable species: tertiary or resonance stabilized carbocation, and $\mathrm{X}$ an electronegative element. It is also amply demonstrated that, if a more stable cationic product can be produced, the $\mathrm{C}-\mathrm{XH}^{+}$ dissociation is accompanied by hydride ion or alkyl migration. For example, the computed energy barrier for the direct methanol elimination from protonated cis-2-methyl-1-methoxycyclohexane (cis-1 Scheme 20) to give 1-methyl-cyclohexyl ion $\boldsymbol{a}$ is equal to $82 \mathrm{~kJ} \mathrm{~mol}^{-1}$ (Ari et al., 2003). If the departure of the methanol molecule is assisted by a 1,2-hydride ion migration in a trans coplanar concerted mechanism, the critical energy is reduced to $40 \mathrm{~kJ} \mathrm{~mol}^{-1}$. This pathway, favored by $42 \mathrm{~kJ} \mathrm{~mol}^{-1}$ with respect to the direct methanol loss gives ride to the more stable 2-methylcyclohexyl ion $\boldsymbol{a}^{\prime}$.

The lowest energy pathway for methanol elimination from the trans-2-methyl-1-methoxycyclohexane isomer is even more complicated. The first step is an isomerization of trans -1 to 2 by a concerted shift of methanol and ring contraction, the second step is a methanol elimination assisted by a 1,2-hydride ion migration as described in Scheme 21.

The $\mathrm{C}-\mathrm{XH}^{+}$dissociation can be also accompanied by internal nucleophilic substitution if another nucleophilic group is present in the molecule. This has been observed for diols, diamines and aminoalcohols. 1,4-Aminobutanol is a typical example of such behavior. The protonated forms of this molecule expel competitively a molecule of ammonia and a molecule of water, the former reaction being favored at low internal energy of the precursor ions (Bouchoux et al., 2002). Collisional activation experiments demonstrate that the product ions are protonated tetra hydro-furan and protonated pyrollidine, respectively thus demonstrating the intramolecular nucleophilic substitutions depicted in Scheme 22. In addition, molecular orbital calculations show that, even though the products of the water loss are the lowest in energy, their formation is ampered by the passage trough the high energy oxygen protonated form of 1,4-aminobutanol.

The $\boldsymbol{b}$ ions of oxazolone structure are formed from protonated peptides by a similar nucleophilic cyclization (Scheme 23; Paizs \& Suhai, 2005). Nucleophilic attack by a carbonyl oxygen of the carbon of the $N$-terminal neighbor carbonyl leads to the formation of a protonated oxazolone derivative: the ion $\boldsymbol{b}$.

The weakening of the $\mathrm{C}-\mathrm{X}$ bond may also give rise to an elongation without bond cleavage leading to the formation of weakly bound ion-neutral complex from which new reactions may take place such as $\mathrm{H}$ exchange, tautomerism or nucleophilic

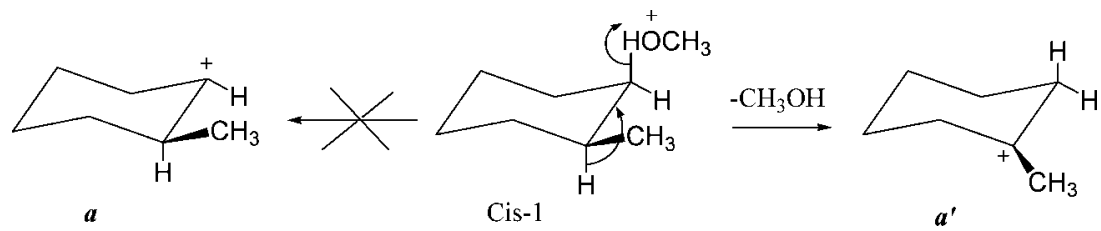

SCHEME 20.

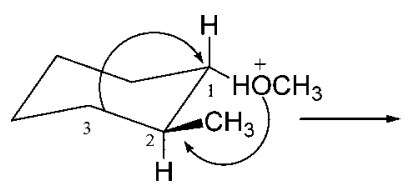

Trans-1

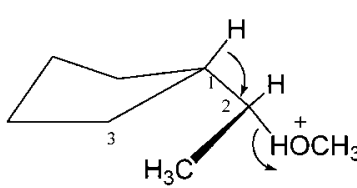

2

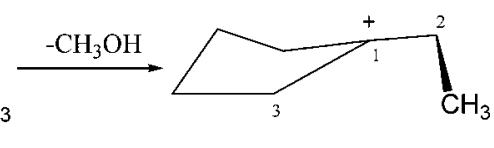

$b$

SCHEME 21. 


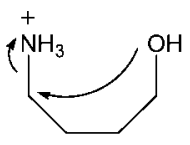

0

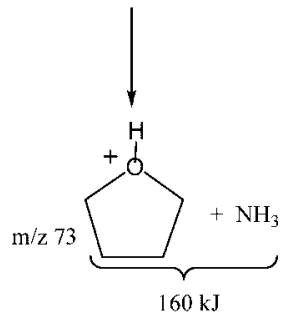

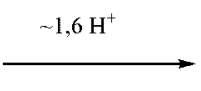

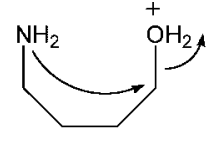

$170 \mathrm{~kJ}$

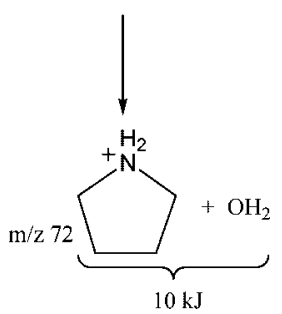

SCHEME 22.

substitution. In the case of the peptide fragmentation presented in Scheme 23, the intermediate ion neutral complex between $\boldsymbol{b}$ and the neutral amine residual, allows a proton exchange and the competitive formation of the $\boldsymbol{y}^{\prime \prime}$ fragment ions.

\section{Tautomerism Induced by the Protonation}

a. Proton transport catalysis. This concept relies to reactions where a proton is translocated inside a cation by means of a neutral partner acting as a formal transporter (Bohme, 1992). The $\mathrm{HOC}^{+} / \mathrm{HCO}^{+}$isomerism is a classical example of such a reaction (Chalk \& Radom, 1997; Collins \& Radom, 2003; Fridgen \& Holmes, 2004). The critical energy for the direct rearrangement of isoformyl $\mathrm{HOC}^{+}$cation to its more stable isomer, the formyl $\mathrm{HCO}^{+}$cation, is approximately $160 \mathrm{~kJ} \mathrm{~mol}^{-1}$. It has been demonstrated that this energy barrier is considerably reduced when a suitable molecule $\mathrm{X}$ intervenes during the overall process (102):

$$
\mathrm{X}+\mathrm{HOC}^{+} \rightarrow\left[\mathrm{XH}^{+} \ldots \mathrm{OC}\right] \rightarrow\left[\mathrm{XH}^{+} \ldots \mathrm{CO}\right] \rightarrow \mathrm{X}+\mathrm{HCO}^{+}
$$

The proton affinity of $\mathrm{X}$ is of crucial importance because the complexation of $\mathrm{X}$ with $\mathrm{HOC}^{+}$or $\mathrm{HCO}^{+}$weakens the bond between $\mathrm{H}$ and $\mathrm{CO}$ as explicited in Equation (102). A systematic study shows that if $\mathrm{X}$ has a proton affinity between that of $\mathrm{O}$ and $\mathrm{C}$ of $\mathrm{CO}$ the barrier for rearrangement completely disappears (Chalk \& Radom, 1997). Other examples of rearrangements of protonated or cationized species involving assisted proton (or even alkyl cation) transport have been reported so far, from small species $\mathrm{HNNO}^{+} / \mathrm{NNOH}^{+}$(Ferguson, 1989), $\mathrm{CH}_{3} \mathrm{CO}^{+} / \mathrm{CH}_{3} \mathrm{OC}^{+}$ (Cunje et al., 1998) to 2-tetrahydropyranol, a model for the study of sugar epimerization (Morpurgo et al., 2000).

b. Generalized tautomerism. Keto/enol tautomerism may be initiated by a proton transfer reaction from an enolizable position to a carbonyl oxygen such as given by Equation (103):

$$
(\mathrm{R})_{2} \mathrm{CO}+\mathrm{CH}_{3}-\mathrm{C}^{+}(\mathrm{OH}) \mathrm{R}^{\prime} \rightarrow(\mathrm{R})_{2} \mathrm{COH}^{+}+\mathrm{CH}_{2}=\mathrm{C}(\mathrm{OH}) \mathrm{R}^{\prime}
$$

If both functions are located on a same molecular species, the proton transfer may be realized by a mechanism formally analogue to the proton transport catalysis evoked above or by other indirect processes. In the example provided by 2,3butanedione (Scheme 24), which is more stable in its diketonic form $\mathbf{I a}$ when unprotonated and in its keto-enol form $\mathbf{1} \boldsymbol{b} \boldsymbol{H}^{+}$when protonated (Akrour et al., 2007), tautomerization occurs within the approach complex by a 1,4-hydrogen migration. The protonated species $\mathrm{BH}^{+}$does not transport the proton but it activates one of the $\mathrm{C}-\mathrm{H}$ bond of 2,3-butanedione by protonating the adjacent carbonyl oxygen. After the 1,4-hydrogen migration leading complex $b$, a rotation around the central $\mathrm{CC}$ bond leads to the complex $c$ which consists in the most stable protonated form $\mathbf{1 b} \mathbf{H}^{+}$in interaction with $\mathrm{B}$.

It is known that, in the gas-phase, aminoacids are more stable in their covalent form $\mathrm{H}_{2} \mathrm{NCHRCO}_{2} \mathrm{H}$ than in their zwitterionic form $\mathrm{H}_{3} \mathrm{~N}^{+} \mathrm{CHRCO}_{2}{ }^{-}$(Harrison, 1997). For example, quantum chemistry calculations indicate that the isolated zwitterionic<smiles>CCCCNC(=O)C(CC)NC(=O)CCCC</smiles><smiles>[R]C1N=C(C[CH2-])OC1=O</smiles><smiles>[R]C1N=C(CC)OC1=O</smiles>

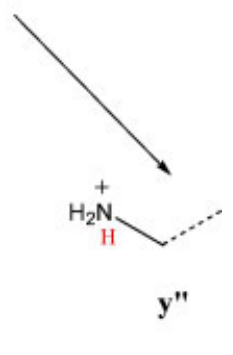

SCHEME 23. 
<smiles>CC(=O)C(=O)OCC(=O)OCc1ccccc1</smiles><smiles>CCC(=O)C(C)=O</smiles>

$1 \mathbf{a}$

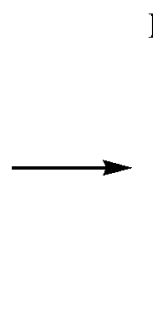

B

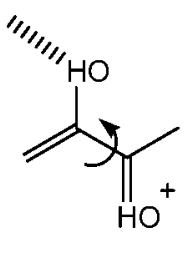

complex $b$

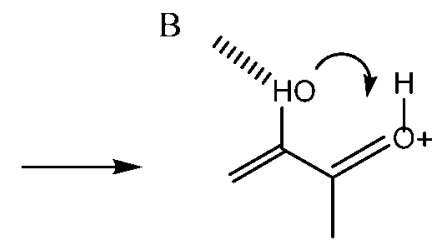

complex $c$<smiles>C=C(O)C(C)=[O+]I</smiles>

$1 \mathbf{b H}^{+}$

SCHEME 24.

structure of glycine $\mathrm{H}_{3} \mathrm{~N}^{+} \mathrm{CH}_{2} \mathrm{CO}_{2}{ }^{-}$is not a minimum in the potential energy surface and evolves spontaneously by a 1,4-H shift toward its neutral $\mathrm{H}_{2} \mathrm{NCH}_{2} \mathrm{CO}_{2} \mathrm{H}$ form, situated $\sim 70 \mathrm{~kJ} \mathrm{~mol}^{-1}$ below the switterion (Jenssen \& Gordon, 1995; Nagaoka, Okuyama-Yoshida, \& Yamabe, 1998). By contrast, in aqueous solution, the reverse stability order is observed and the question of as to whether a comparable situation exists inside protonated clusters may be asked. Indeed several recent studies show that the two forms, covalent and zwitterionic, may intervene in clusters (Julian, Hodyss, \& Beauchamp, 2001; Raspopov \& McMahon, 2005; Wu \& McMahon, 2005). The thermochemistry of gas-phase interaction between protonated glycine and ammonia has been studied both experimentally by high-pressure mass spectrometry and theoretically ( $\mathrm{Wu} \&$ McMahon, 2005). The experimental binding energy for association between $\mathrm{GlyH}^{+}$and ammonia is $97 \mathrm{~kJ} \mathrm{~mol}^{-1}$. Quantum chemical calculations show that essentially two structures, of comparable stabilities, are compatible with this clustering energy (GNcov and GNzw, Fig. 36). The first one is a complex between covalent glycine and protonated ammonia while the second is a complex between the zwitterionic glycine with protonated ammonia. The two hydrogen bonds in this latter structure,

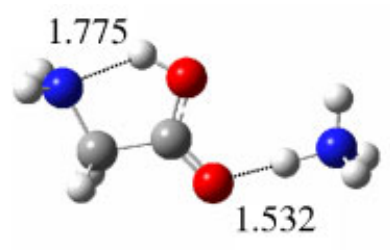

GNcov

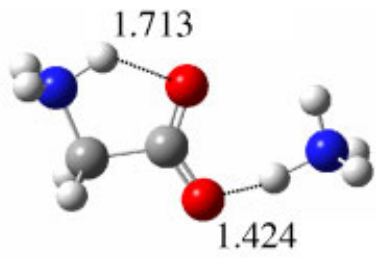

GNzw
FIGURE 36. B3LYP/6-311+G(d,p) optimized geometries of the two most stable complexes between glycine and ammonium cation. [Color figure can be viewed in the online issue, which is available at www.interscience.wiley.com.]
GNzw, appear to be particularly favorable since the acceptor moiety is, formally, negatively charged. As a consequence, the bond length between the ammonium hydrogen and carboxylate oxygen is significantly reduced relative to the analogous bond in GNcov. Interestingly enough, the 1,4 internal proton transfer separating GNcov and GNzw is associated with a negligible critical energy of less than $2 \mathrm{~kJ} \mathrm{~mol}^{-1}$. No doubt that, after comparing this critical energy to the binding energy of the two complexes, a statistical mixture of isomers GNcov and GNzw will be present under thermal equilibrium conditions.

\section{Ring Forming and Ring Opening Induced by Protonation}

a. Cyclization. Formation of a strong intramolecular hydrogen bond in protonated bifunctional molecules is one of the major source of cyclization induced by the protonation in the gas-phase as it has been underlined above and as it will be exemplified in the forthcoming parts of this review. Cyclization by formation of a covalent bond by a nucleophilic attack may also accompany the protonation process.

One example is provided by 2,5-hexanedione which, upon protonation may give rise to an internal hydrogen bonded structure $\boldsymbol{a H}$ or to the furanoyl structure $\boldsymbol{a C}$ (Scheme 25). Quantum chemical calculation conducted at the G2MP2 level shows that the latter ion is more stable than the former by $10 \mathrm{~kJ} \mathrm{~mol}^{-1}$ (Akrour et al., 2007). The proton affinity calculated in the hypothesis of the formation of $\boldsymbol{a C}, 892 \mathrm{~kJ} \mathrm{~mol}^{-1}$, is in complete agreement with the experimental value obtained from variable temperature high-pressure mass spectrometry experiments (Mautner, 1984).

b. Ring opening. During protonation of cyclic compounds, the $\mathrm{C}-\mathrm{X}$ bond elongation may lead to a ring opening without separation of two fragments. It has been for example observed that protonation of lactones occurs preferentially to the carbonyl 


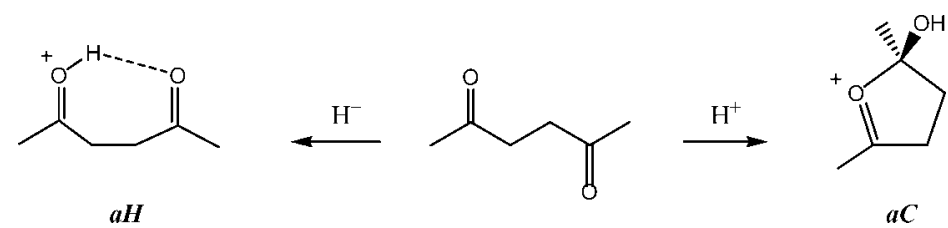

SCHEME 25.

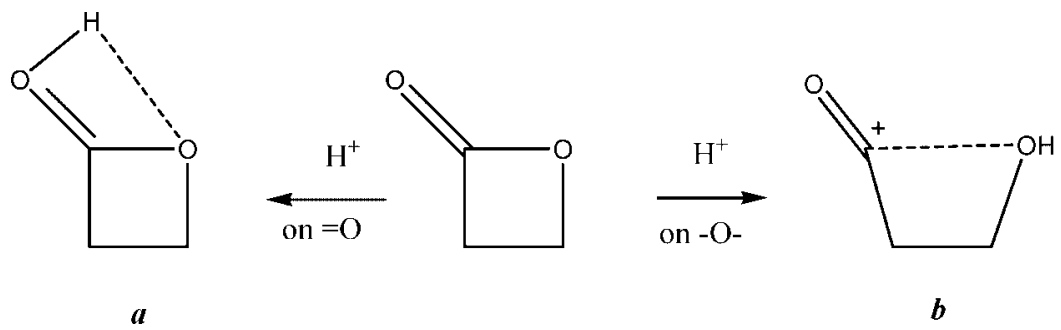

SCHEME 26.<smiles>O=C1CCC(=O)O1</smiles>
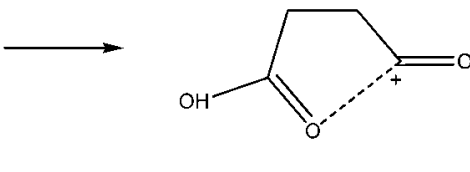

$\mathbf{H}^{\prime}$

SCHEME 27.

oxygen but that protonation at the oxygen ring may give rise to an open form of significant stability (Bouchoux et al., 1995). The most spectacular case is propiolactone for which the two protonated forms are of comparable stabilities ( $\boldsymbol{a}$ and $\boldsymbol{b}$ Scheme 26). From a general point of view, lactones exhibit larger basicities than esters due to a favorable interaction between the incoming proton and the ether-like oxygen, this is also the case for structure $\boldsymbol{a}$. It is also noteworthy that the ring opening is not complete in structure $\boldsymbol{b}$ since an electrostatic interaction between the hydroxyl oxygen and the carbon of the acylium-like carbon stabilizes this conformation.

Similarly, protonation of cyclic anhydrides at one of the carbonyl oxygen results in the fission of the farthest $\mathrm{C}-\mathrm{O}$ bond as exemplified with succinic anhydride in Scheme 27 (Bouchoux et al., 1998). This is supported by molecular orbital calculations but also by the experimental determination of a sizeable entropy gain (up to $30 \mathrm{~J} \mathrm{~mol}^{-1} \mathrm{~K}^{-1}$ ) upon protonation. This bond cleavage, attested by charge density calculations, is however not complete. The conservation of a semi-cyclic arrangement allows a favorable electrostatic interaction between the positive charge of the incipient acylium carbon and the carbonyl oxygen of the newly formed carboxylic acid group. The semi-cyclic form is stabilized by ca. $50 \mathrm{~kJ} \mathrm{~mol}^{-1}$ with respect to its open chain conformer.

Ring opening upon protonation is also predicted for heterocyclic aromatic molecules such as $1 \mathrm{H}$ - or $2 \mathrm{H}-1,2,3-$ triazoles (Kabli et al., 2006) and 1H-tetrazoles (Esseffar et al., 2002).

\section{Internal Nucleophilic Substitution Inside Protonated Clusters}

It is known for a long time that proton bound pairs of alcohols may eliminate a water molecule (Beauchamp \& Caserio, 1972). Numerous experimental and computational studies have established that this elimination reaction is preceded by a nucleophilic substitution occurring in a rearranged cluster (Bouchoux \& Choret, 1997; Fridgen, Keller, \& McMahon, 2001; Fridgen \& McMahon, 2002, 2003). The general reaction routes proposed to explain the observed results are summarized in Scheme 28. It involves a $\mathrm{R}^{+}$cation transfer $\left(\mathrm{S}_{\mathrm{N}} 2\right.$ reaction) between complexes $\boldsymbol{a}_{\boldsymbol{C}}$ and $\boldsymbol{b}_{\boldsymbol{C}}$ with a possible passage through a proton bound dimer $\boldsymbol{a}_{\boldsymbol{H}}$, this latter structure being significantly more stable than $\boldsymbol{a}_{\boldsymbol{C}}$.

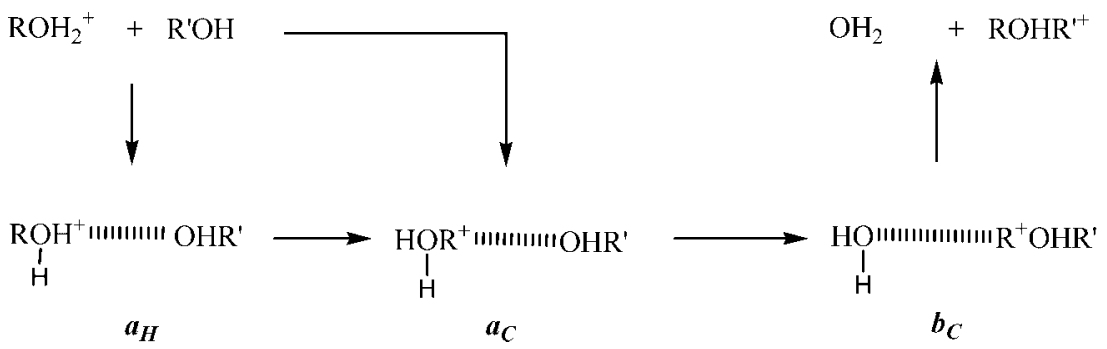

SCHEME 28. 
The barrier for the alkyl cation transfer is sensitive to the size of the migrating group, it is lowered for the larger alkyl groups (Fridgen \& McMahon, 2003). Moreover linear relationships have been devised for evaluating the relative energies of intermediate $\boldsymbol{a}_{\boldsymbol{C}}$ and $\mathrm{S}_{\mathrm{N}} 2$ transition state as a function of the proton affinities of the precursor alcohols (Fridgen \& McMahon, 2003; Grabowy \& Mayer, 2005).

Nitrile-alcohol protonated clusters exhibit also facile water loss thus demonstrating a comparable rearrangement involving isomerization of the most stable approach complex $\mathrm{RCN} \cdots \mathrm{H}+\cdots \mathrm{O}(\mathrm{H}) \mathrm{R}^{\prime}$ to $\mathrm{RCN} \cdots \cdot \mathrm{R}^{\prime} \mathrm{OH}_{2}{ }^{+}$followed by a $\mathrm{S}_{\mathrm{N}} 2$ step $\mathrm{RCN} \cdots \mathrm{R}^{\prime} \mathrm{OH}_{2}{ }^{+} \rightarrow \mathrm{RCNR}^{\prime}{ }^{+} \ldots \mathrm{OH}_{2}$ (Mayer, 1999; Ochran, Annamali, \& Mayer, 2000; Ochran \& Mayer, 2001). More recently, a similar rearrangement has been proposed to explain the $\mathrm{NO}_{2} \mathrm{H}$ loss from nitroalkane proton bound pairs (Poon \& Mayer, 2006).

\section{CONCLUSION}

This first part of a review on the basicity of polyfunctional molecules has been centered on the concepts and methods allowing the understanding of the protonation of mono- and polyfunctional isolated molecules. A survey of the experimental and theoretical methods of determination of protonation thermochemistry is proposed and some insight on the structural changes induced by the protonation of isolated molecules, but also hydrogen bonded clusters, are given. It has been shown that experimental methods (equilibrium, kinetic, thermokinetic) allow generally accurate measurements of gas-phase basicities. However, each have limitations, in particular the equilibrium method is not usable for non-volatile compounds while the kinetic and thermokinetic methods need still improvement to correctly treat the protonation entropy. Theory may provide correct proton affinity estimates and more or less accurate protonation entropy if the size of the molecule is not too large.

Polyfunctional molecules are characterized by the possibilities of interfunctional interactions which, upon protonation, may be dramatically increased and lead to considerable structural changes. These modifications have serious effects on the proton affinity, protonation entropy and consequently gas-phase basicity of these molecules. The large entropy change frequently observed when protonating a polyfunctional molecule is perhaps the most difficult quantity to handle both experimentally and theoretically. A careful examination of the available data is thus necessary.

\section{REFERENCES}

Abboud JLM, Canada T, Homan H, Notario R, Cativiela C, Diaz de Villegas MD, Bordeje MC, Mo O, Yanez M. 1992. Gas-phase basicities of $\beta$-lactams and azetidines, cyclisation effects: An experimental and theoretical study. J Am Chem Soc 114:4728-4736.

Abboud JLM, Notario R, Ballesteros E, Herreros M, Mo O, Yanez M, Elguero J, Boyer G, Claramunt R. 1994. Dissociative attachment of proton to 1fluoro- and 1-chloroadamantane in the gas-phase. J Am Chem Soc 116:2486-2492.
Adams NG, Smith D. 1988. Flowing afterglow and SIFT. In: Farrar JM, Saunders W, editors. Techniques for the study of ion-molecule reactions. New York: John Wiley \& Sons. pp. 165-220.

Akrour A, Chikh Z, Djazi F, Elbannay M, Berruyer F, Bouchoux G. 2007. Gas phase protonation of $\alpha, \beta$ and $\gamma$-dicarbonyls: Thermochemistry and structures. Int J Mass Spectrom (in press).

Alcami M, Mo O, Yanez M, Abboud JLM, Elguero J. 1990. Bond activation by protonation in the gas-phase. Chem Phys Lett 172:471-477.

Alcami M, Mo O, Yanez M. 2001. Computational chemistry: A useful (sometimes mandatory) tool in mass spectrometry studies. Mass Spectrom Rev 20:195-245.

Alcami M, Mo O, Yanez M. 2002. Modeling intrinsic basicities and acidities. J Phys Org Chem 15:174-186.

Alkorta I, Elguero J, Mo O, Yanez M, Del Bene J. 2005. Are resonanceassisted hydrogen bonds "resonance assisted"? A theoretical NMR study. Chel Phys Lett 411:411-415.

Ari JB, Karni M, Apeloig Y, Mandelbaum A. 2003. The role of hydride migration in the mechanism of alcohol elimination from protonated ethers upon chemical ionisation. Experiment and theory. Int J Mass Spectrom 228:297-306.

Armentrout P. 2000. Entropy measurements and the kinetic method: A statistically meaningful approach. J Am Soc Mass Spectrom 371-379.

Aubry C, Holmes JL. 1998. Butyl cations and their gas-phase dissociation chemistry: Uniting experiments with theory. J Phys Chem A 102:64416447.

Aubry C, Holmes JL. 2000. Correlating thermochemical data for gas-phase ion chemistry. Int J Mass Spectrom 200:277-284.

Aue DH, Bowers MT. 1979. Stabilities of positive ions from equilibrium gasphase basicity measurements. In: Bowers MT, editor. Gas phase ion chemistry, Vol. 2. New York: Academic press. pp. 1-51.

Aue DH, Webb HM, Bowers MT. 1973. Quantitative evaluation of intramolecular strong hydrogen bonding in the gas-phase. J Am Chem Soc 95:2699-2701.

Aue DH, Webb HM, Bowers MT. 1976. Quantitative proton affinities, ionization energies and hydrogen affinities of alkylamines. J Am Chem Soc 98:311-317.

Aue DH, Guidoni M, Betowski LD. 2000. Ab initio calculated gas-phase basicities of polynuclear aromatic hydrocarbons. Int J Mass Spectrom 201:283-295.

Bader RFW. 1990. Atoms in molecules. A quantum theory. Oxford: Clarendon Press.

Baer T, Hase WL. 1996. Unimolecular reaction dynamics. Theory and experiments. New York: Oxford University Press.

Barker JR, Shovlin CN. 2004. An approximation for hindered rotor state energies. Chem Phys Lett 383:203-207.

Bartmess JE. 1989. Gas-phase equilibrium affinity scales and chemical ionization mass spectrometry. Mass Spectrom Rev 8:297-343.

Bartmess JE. 1994. Thermodynamic of the electron and the proton. J Phys Chem 98:6420-6424.

Bartmess JE. 2003. The bracketing method for determining the thermochemistry of ion-molecule reactions. Encyclopedia of mass spectrometry, Vol. 1. Amsterdam: Elsevier. pp. 315-319.

Beauchamp JL, Caserio MC. 1972. Ion-molecule reactions of 2-butanol by ion cyclotron resonance spectroscopy. J Am Chem Soc 94:2638-2646.

Bell RP. 1973. The proton in chemistry, 2nd edition. London: Chapman and Hall.

Benoit FM, Harrison AG. 1977. Predictive value of proton affinity. Ionisation energy correlations involving oxygenated molecules. J Am Chem Soc 99:3980-3984.

Benson SW. 1976. Thermochemical kinetics, 2nd edition. New York: John Wiley \& Sons.

Bierbaum VM. 2003. Flow-tubes. Encyclopedia of mass spectrometry, Vol. 1. Amsterdam: Elsevier. pp. 98-109. 
Boggs PT, Byrd RH, Rogers JE, Schnabel RB. 1992. ODRPACK version 2.01 Software for weighted orthogonal distance regression. Report NISTIR 92-4834, National Institute of Standards and Technology, Gaithersburg, MD.

Bohme. 1992. Proton transport in the catalyzed gas-phase isomerization of protonated molecules. Int J Mass Spectrom 115:95-110.

Bohme DK, MacKay GI, Schiff HI. 1980. Determination of proton affinities from the kinetics of proton transfer reactions VII The proton affinities of $\mathrm{O}_{2}, \mathrm{H}_{2}, \mathrm{Kr}, \mathrm{O}, \mathrm{N}_{2}, \mathrm{Xe}, \mathrm{CO}_{2}, \mathrm{CH}_{4}, \mathrm{~N}_{2} \mathrm{O}$ and CO. J Chem Phys 73:49764986.

Bojesen G, Breindahl T. 1994. On the proton affinity of some a-aminoacids and the theory of the kinetic method. J Chem Soc Perkin Trans 2:1029_ 1037.

Bordeje MC, Mo O, Yanez M, Herreros M, Abboud JLM. 1993. Cyclization effects on the gas-phase basicities of esters and ethers. An experimental and MO study. J Am Chem Soc 115:7389-7396.

Bouchoux G. 2006a. Microcanonical modeling of the thermokinetic method. J Phys Chem A 110:8259-8265.

Bouchoux G. 2006b. Evaluation of the protonation thermochemistry obtained by the extended kinetic method. J Mass Spectrom 41:1006-1013.

Bouchoux G, Berruyer-Penaud F. 2003c. Protonation thermochemistry of $\alpha, \omega$-alkanediols in the gas-phase: A theoretical study. J Phys Chem A 107:7931-7937.

Bouchoux G, Buisson DA. 2006. Gas phase basicity of $\mathrm{X}\left(\mathrm{CH}_{2}\right)_{3} \mathrm{Y}$ $\left(\mathrm{X}, \mathrm{Y}=\mathrm{OH}, \mathrm{NH}_{2}\right)$ by the thermokinetic method. Int J Mass Spectrom 249-150:412-419.

Bouchoux G, Choret N. 1997. Reactions between protonated and neutral methanol in the gas-phase: an ab initio molecular orbital study. Rapid Commun Mass Spectrom 11:1799-1807.

Bouchoux G, Houriet R. 1984. Ring size effect on the gas-phase protonation of cyclic ketones. Tetrahedron Lett 25:5755-5758.

Bouchoux G, Drancourt D, Leblanc D, Yanez M, Mo O. 1995. Gas-phase basicities of lactones. New J Chem 19:1243-1257.

Bouchoux G, Salpin JY, Leblanc D. 1996. A relationship between the kinetics and thermochemistry of proton transfer reactions in the gas phase. Int J Mass Spectrom 153:37-48.

Bouchoux G, Gal JF, Maria PC, Szulejko JE, McMahon TB, Tortajada J, Luna A, Yanez M, Mo O. 1998. Gas-phase basicities of acid anhydrides. J Phys Chem A 102:9183-9192.

Bouchoux G, Yanez M, Mo O. 1999. Isomerization and dissociation processes of protonated benzene and protonated fulvene in the gas phase. Int $\mathbf{J}$ Mass Spectrom 185/186/187:241-251.

Bouchoux G, Caunan F, Leblanc D, Nguyen MT, Salpin JY. 2001a. Gas phase basicities of ethyl halides. Chem Phys Chem 1:604-610.

Bouchoux G, Leblanc D, Sablier M. 2001b. Determination of ionization energies by the thermokinetic method. Int J Mass Spectrom 210/211: 189-201.

Bouchoux G, Choret N, Berruyer-Penaud F. 2001c. Protonation thermochemistry of $\alpha, \omega$-alkyldiamines in the gas-phase: A theoretical study. J Phys Chem A 107:7931-7937.

Bouchoux G, Choret N, Berruyer-Penaud F, Flammang R. 2002. Thermochemistry and unimolecular reactivity of protonated $\alpha, \omega-$ aminoalcohols in the gas-phase. Int J Mass Spectrom 217:195230.

Bouchoux G, Djazi F, Gaillard F, Vierezet D. 2003a. Application of the kinetic method to bifunctional bases. MIKE and CID-MIKE test cases. Int J Mass Spectrom 227:479-496.

Bouchoux G, Buisson DA, Bourcier S, Sablier M. 2003b. Application of the kinetic method to bifunctional bases. ESI tandem quadrupole experiments. Int J Mass Spectrom 228:1035-1054.

Bouchoux G, Sablier M, Berruyer-Penaud F. 2004. Obtaining thermochemical data by the extended kinetic method. J Mass Spectrom 39:986-997.
Bouchoux G, Leblanc D, Bertrand W, McMahon TB, Szulejko JE, BerruyerPenaud F, Mo O, Yanez M. 2005. Protonation thermochemistry of selected hydroxy and methoxycarbonyl molecules. J Phys Chem A 109:11851-11859.

Bowers MT, Aue DH, Webb HM, McIver RT. 1971. Equilibrium constants for gas-phase ionic reactions. Accurate determination of relative proton affinities. J Am Chem Soc 93:4314-4315.

Boyd SL, Boyd RJ. 1997. A theoretical study of the effects of protonation and deprotonation on bond dissociation energies of second row elements: Comparison with first row elements. J Am Chem Soc 119:4214-4219.

Boyd SL, Boyd RJ. 1999. A theoretical study of the effects of protonation and deprotonation on bond dissociation energies of third row elements: Homolytic versus heterolytic cleavage. J Phys Chem A 103:70877093.

Boyd SL, Boyd RJ, Bessonette PW, Kerdraon DI, Aucoin NT. 1995. A theoretical study of the effects of protonation and deprotonation on bond dissociation energies. J Am Chem Soc 117:8816-8822.

Brönsted JN. 1928. Acid and basic catalysis. Chem Rev 5:231-338.

Brown JR, Schwerdtfeger P, Schröder D, Schwarz H. 2002. Experimental and theoretical studies of diatomic gold halides. J Am Soc Mass Spectrom $13: 485-492$

Burk P, Koppel IA, Koppel I, Leito I, Travnikova O. 2000. Critical test of performance of B3LYP functional for prediction of gas phase acidities and basicities. Chem Phys Lett 323:482-489.

Campbell S, Beauchamp JL, Rempe M, Lichtenberger DL. 1992. Correlations of lone pair ionization energies with proton affinities of amino acids and related compounds. Site specificity of protonation. Int J Mass Spectrom Ion Proc 117:83-99.

Cao J, Holmes JL. 1999. Determining the proton affinities of nitriles by the kinetic method. Eur J Mass Spectrom 5:19-22.

Cao J, Aubry C, Holmes JL. 2000. Proton affinities of simple amines; entropies and enthalpies of activation and their effect on the kinetic method for evaluating proton affinities. J Phys Chem 104:10045-10052.

Carroll TX, Smith SR, Thomas TD. 1974. Correlation between proton affinity and core-electron ionization potentials for double-bonded oxygen. Site of protonation in esters. J Am Chem Soc 97:659-660.

Cerda BA, Wesdemiotis C. 1996. $\mathrm{Li}^{+}, \mathrm{Na}^{+}$, and $\mathrm{K}^{+}$Binding to the DNA and RNA Nucleobases. Bond energies and attachment sites from the dissociation of metal ion-bound heterodimers. J Am Chem Soc 118:11884-11892.

Chalk AJ, Radom L. 1997. Proton-transport catalysis: A systematic study of the rearrangement of the isoformyl cation to the formyl cation. J Am Chem Soc 119:7573-7578.

Chan B, Del Bene JE, Elgero J, Radom L. 2005. On the relationship between the preferred site of hydrogen bonding and protonation. J Phys Chem A 109:5509-5517.

Chen CC, Bozzelli JW. 2003. Structures, intramolecular rotation barriers and thermochemical properties of methyl, ethyl, methyl isopropyl, and methyl tert-butyl ethers and the corresponding radicals. J Phys Chem A 107:4531-4546.

Chiavarino B, Crestoni ME, Fornatini S. 2003. Gas phase regioselectivity in the deprotonation of $p$-cresol radical cation. Chem Phys Lett 372:183186.

Chuang YY, Truhlar D. 2000. Statistical thermodynamics of bond torsional modes. J Chem Phys 112:1221-1228.

Chuchani G, Mishima M, Notario R, Abboud JL. 1999. Structural effects on gas phase reactivities. Adv Quant Struct Property Relationships 2:35126.

Cioslowsky J, Scott AP, Radom L. 1997. Catastrophes, bifurcations and hysteresis loops in torsional potentials of internal rotations in molecules. Mol Phys 91:413-420.

Collins MA, Radom L. 2003. Proton-transport catalysis, proton abstraction and proton exchange in $\mathrm{HF}+\mathrm{HOC}^{+}$and $\mathrm{H}_{2} \mathrm{O}+\mathrm{HOC}^{+}$and analogous deuterated reactions. J Chem Phys 118:6222-6229. 
Cooks RG, Kruger TL. 1977. Intrinsic basicity determination using metastable ions. J Am Chem Soc 99:1279-1281.

Cooks RG, Wong PSH. 1998. Kinetic method of making thermochemical determinations: Advances and applications. Account Chem Res 31: 379-386.

Cooks RG, Patrick JS, Kotaho T, McLuckey SA. 1994. Thermochemical determinations by the kinetic method. Mass Spectrom Rev 13:287339.

Cooks RG, Koskinen JT, Thomas PD. 1999. The kinetic method of making thermochemical determinations. J Mass Spectrom 34:85-92.

Coote ML, Pross A, Radom L. 2003. Variable trends in R-X bond dissociation energies $(\mathrm{R}=\mathrm{Me}$, Et, I-Pr, t-Bu). Org Lett 5:4689-4692.

Craig SL, Zhong M, Choo B, Brauman JI. 1997. Branching ratio in activated systems. J Phys Chem A 101:19-24.

Cunje A, Rodriquez CF, Bohme DK, Hopkinson AC. 1998. Interconversion of $\mathrm{ROC}^{+}$and $\mathrm{RCO}^{+}\left(\mathrm{R}=\mathrm{H}\right.$ and $\left.\mathrm{CH}_{3}\right)$ : Gas-phase catalysis by argon and dinitrogen. J Phys Chem A 102:478-483.

Curtiss LA, Blander M. 1988. Thermodynamic properties of gas-phase hydrogen bonded complexes. Chem Rev 88:827-841.

Curtiss LA, Pople JA. 1988. Theoretical study of structures and energies of acetylene, ethylene and vinyl radical and cation. J Chem Phys 88:74057409.

Curtiss LA, Raghavachari K. 2002. Gaussian-3 and related methods for accurate thermochemistry. Theor Chem Acc 108:61-70.

Curtiss LA, Raghavachari K, Trucks GW, Pople JA. 1991. A Gaussian-2 theory for molecular energies of first- and second-row compounds. J Chem Phys 94:7221-7230.

Curtiss LA, Raghavachari K, Redfern PC, Pople JA. 2000. Assessment of Gaussian-3 and density functional theories for a larger experimental set. J Chem Phys 112:7374-7383.

Da Silva G, Kim CH, Bozzelli JW. 2006. Thermochemical properties (enthalpy, bond energy, entropy, heat capacity) and internal rotor potentials of vinyl alcohol, methylvinyl ether and their corresponding radicals. J Phys Chem A 110:7925-7934.

Davalos JZ, Herrero R, Quintanilla E, Jimenez P, Gal JF, Maria PC, Abboud JL. 2006. Ab initio and experimental thermodynamic and kinetic study of proton assisted bond activation in gaseous hydrocarbons: Deconvolution of reaction efficiencies in the case of adamantine. Chem Eur J 12:5505-5513.

Davidson WR, Sunner J, Kebarle P. 1979. Hydrogen bonding of water to onium ions. Hydration of substituted pyridinium ions and related systems. J Am Chem Soc 101:1675-1680.

Davis DW, Rabalais JW. 1974. Model for proton affinities and inner-shel electron binding energies based on the Hellmann-Feynman theorem. J Am Chem Soc 96:5305-5310.

Deakyne CA. 2003. Proton affinities and gas-phase basicities: Theoretical methods and structural effects. Int J Mass Spectrom 227:601616

Decker BK, Adams NG, Babcock LM. 1999. Gas-phase reactivity of $\mathrm{HS}_{2} \mathrm{H}^{+}$ and $\mathrm{S}_{2}{ }^{+}$: An investigation of the gas basicity and proton affinity of $\mathrm{HS}_{2}$. Int J Mass Spectrom 185/186/187:727-743.

Decouzon M, Gal JF, Herreros M, Maria PC, Murrell J, Todd JFJ. 1996. On the use of the kinetic method for the determination of proton affinities by Fourier transform ion cyclotron resonance mass spectrometry. Rapid Commun Mass Spectrom 10:242-245.

Deshmukh MM, Gadre SR, Bartolotti LJ. 2006. Estimation of intramolecular hydrogen bond energy via molecular tailoring approach. J Phys Chem A 110:12519-12523.

Desiraju GR, Steiner T. 1999. The weak hydrogen bond in structural chemistry and biology. New York: Oxford University Press.

DeYonker NJ, Cundari TR, Wilson AK. 2006. The correlation consisten approach (ccCA): An alternative to the Gaussian- $n$ methods. J Chem Phys 124:114104
DeYonker NJ, Grimes T, Yockel S, Dinescu A, Mintz B, Cundari TR, Wilson AK. 2006. The correlation consistent approach: Application to the G3/ 99 test set. J Chem Phys 125:104111.

Dopfer O, Solca N, Lemaire J, Maitre P, Crestoni ME, Fornarini S. 2005. Protonation sites of isolated fluorobenzene revealed by IR spectroscopy in the fingerprint range. J Phys Chem 109:7881-7887.

Drahos L, Vekey K. 1999. How closely related are the effective and real temperatures. J Mass Spectrom 34:79-84.

Drahos L, Vekey K. 2003. Entropy evaluation using the kinetic method: Is it feasible? J Mass Spectrom 38:1025-1042.

Drahos L, Peltz C, Vekey K. 2004. Accuracy of enthalpy and entropy determination using the kinetic method: Are we approaching a consensus? J Mass Spectrom 38:1016-1024.

East ALL, Radom ML. 1997. Ab initio statistical thermodynamic model for the computation of third law entropies. J Chem Phys 106:6655-6674.

East ALL, Smith BJ, Radom L. 1997. Entropies and free energies of protonation and proton transfer reactions. J Am Chem Soc 119:90149020 .

El Firdoussi A, Esseffar M, Bouab W, Abboud JLM, Mo O, Yanez M, Ruasse MF. 2005. Density functional theory study of the hydrogen bond interaction between lactones lactams and methanol. J Phys Chem A 109:9141-9148.

Ervin KM. 2000. Microcanonical analysis of the kinetic method. The meaning of the "effective temperature". Int J Mass Spectrom 195/ 196:271-284

Ervin KM. 2001. Experimental techniques in gas phase ion thermochemistry. Chem Rev 101:391-444.

Ervin KM. 2002. Microcanonical analysis of the kinetic method. The meaning of the "apparent entropy". J Am Soc Mass Spectrom 13:435452.

Ervin KM, Armentrout PB. 2004. Systematic and random errors in ion affinities and activation entropies from the extended kinetic method. J Mass Spectrom 39:1004-1015.

Esseffar M, Quintanilla E, Davalos JZ, Abbound JLM, Mo O, Yanez M. 2002. Nitroderivatives of pyrrole, furan and $1 \mathrm{H}$-tetrazole: Ring or nitro bases? New J Chem 26:1567-1574

Exner O. 1988. Correlation analysis of chemical data. New York: Plenum Press.

Fairley DA, Scott GBI, Freeman CG, Maclagan RGAR, McEwan MJ. 1996. Ion-molecule association of $\mathrm{H}_{3} \mathrm{O}^{+}$and $\mathrm{C}_{2} \mathrm{H}_{2}$ : Interstellar $\mathrm{CH}_{3} \mathrm{CHO}$. J Chem Soc Faraday Trans 92:1305-1309.

Ferguson EE. 1989. Reactions of $\mathrm{NNOH}^{+}$and $\mathrm{HNNO}^{+}$ions with $\mathrm{CH}_{4}$ and NO. Chem Phys Lett 156:319-322.

Fridgen TD, Holmes JL. 2004. A study of the methane catalyzed isomerization of $\mathrm{HCO}^{+}$to $\mathrm{HOC}^{+}$and the elimination of methane from metastable metyhoxymethyl cation. Eur J Mass Spectrom 10:747-754.

Fridgen TD, McMahon TB. 2002. Experimental determination of activation energies for gas-phase ethyl and $n$-propyl cation transfer reactions. J Phys Chem A 106:9648-9654.

Fridgen TD, McMahon TB. 2003. Enthalpy barriers for asymmetric SN2 alkyl cation transfer reactions between neutral and protonated alcohols. J Phys Chem A 107:668-675.

Fridgen TD, Keller JD, McMahon TB. 2001. Direct experimental determination of the energy barriers for methyl cation transfer in the reactions of methanol with protonated methanol, protonated acetonitrile and protonated acethaldehyde: A low pressure FTICR study. J Phys Chem A 105:3816-3824.

Gal JF, Maria PC. 1990. Correlation analysis of acidity and basicity: From the solution to the gas phase. Prog Phys Org Chem 17:159-238.

Gal JF, Maria PC, Raczynska ED. 2001. Thermochemical aspects of proton transfer in the gas phase. J Mass Spectrom 36:699-716.

Gilbert RG, Smith SC. 1990. Theory of unimolecular and recombination reactions. Oxford: Blackwell Scientific Publications. 
Gilli G, Gilli P. 2000. Towards an unified hydrogen bond theory. J Mol Struct $552: 1-15$.

Glukhovtsev MN, Bach RD. 1998. Ab initio study on the thermochemistry of vinyl radical and cation. Chem Phys Lett 286:51-55.

Glukhovtsev MN, Pross A, Nicolaides A, Radom L. 1995. Is the most stable gas-phase isomer of the benzenium cation a face-protonated $\pi$ complex? J Chem Soc Chem Commun 2347-2348.

Gordon MS. 2003. Energies and potential energy surfaces: Theoretical methods. In: Gross ML, Caprioli RM, Armentrout P (editors). Encyclopedia of mass spectrometry, Vol. 1. Amsterdam: Elsevier. pp. 47-55.

Gorman GS, Amster IJ. 1993. Kinetic and thermodynamic considerations of the bracketing method: Entropy driven proton transfer reactions in a Fourier transform mass spectrometer. Org Mass Spectrom 28:16021607.

Grabowy JAD, Mayer PM. 2004. Entropy changes in dissociation of proton bound complexes: A variational RRKM study. J Phys Chem A 108: 9726-9732.

Grabowy JAD, Mayer PM. 2005. Predicting ion rearrangement reactions: The energetics of the internal $\mathrm{SN} 2$ reaction in gas-phase proton bond molecular pairs. Can J Chem 83:1864-1871.

Gross ML, Caprioli RM. 2003. In: Armentrout P, editor. The encyclopedia of mass spectrometry. Vol. 1: Theory and ion chemistry. Amsterdam: Elsevier.

Gross ML, Caprioli RM. 2005. In: Nibbering NMM, editor The encyclopedia of mass spectrometry. Vol. 4: Fundamentals of and application to organic (and organometallic) compounds. Amsterdam: Elsevier.

Guthrie JP. 2001. Use of DFT methods for the calculation of the entropy of gas phase organic molecules: An examination of the quality of results from a simple approach. J Phys Chem A 105:8495-8499.

Hahn IS, Wesdemiotis C. 2003. Protonation thermochemistry of $\beta$-alanine. An evaluation of proton affinities and entropies determined by the extended kinetic method. Int J Mass Spectrom 222:465-479.

Hammerum S. 1997. Heats of formation of gas phase ions calculated by composite ab initio procedures. Int J Mass Spectrom Ion Proc 165/ 166:63-69.

Hammerum S. 1999. Heats of formation and proton affinities by the G3 method. Chem Phys Lett 300:529-532.

Hammerum S, Solling TI. 1999. The proton affinities of imines and the heats of formation of immonium ions investigated with composite ab initio methods. J Am Chem Soc 121:6002-6009.

Hammett LP. 1937. The effect of structure upon the reaction of organic compounds. Benzene derivatives. J Am Chem Soc 59:96-103.

Harrison AG. 1992. Chemical ionization mass spectrometry, 2nd edition. Boca Raton: CRC Press.

Harrison AG. 1997. The gas phase basicities and proton affinities of amino acids and peptides. Mass Spectrom Rev 16:201-217.

Harrison AG. 1999. Linear free energy correlations in mass spectrometry. J Mass Spectrom 34:577-589.

Hartmann M, Westmore SD, Radom L. 2001. C-H $\cdots$ X hydrogen bonds of acetylene, ethylene and ethane with first- and second-row hydrides. J Phys Chem A 105:4470-4479.

Hnizdo V, Fedorowicz A, Singh H, Demchuk E. 2003. Statistical thermodynamics of internal rotation in a hindering potential of mean force obtained from computer simulations. J Comput Chem 24:1172-1183.

Holmes JL. 2003. The correlation of thermochemical data. In: Gross ML, Caprioli RM, Armentrout P (editors). Encyclopedia of mass spectrometry, Vol. 1. Amsterdam: Elsevier. pp. 434-440.

Holmes JL, Lossing FP. 1982. Toward a general scheme for estimating the heats of formation of organic ions in the gas-phase. Part II. The effect of substitution at the charge bearing sites. Can J Chem 60:2365-2371.

Holmes JL, Lossing FP. 1991. Ionization energies of homologous organic compounds and correlation with molecular size. Org Mass Spectrom 26:537-541.
Holmes JL, Fingas M, Lossing FP. 1981. Toward a general scheme for estimating the heats of formation of organic ions in the gas-phase. Part I. Odd-electron cations. Can J Chem 59:80-93.

Hunter EP, Lias SG. 1998. Evaluated gas phase basicities and proton affinities of molecules: An update. J Phys Chem Ref Data 27:413-656.

Hunter EP, Lias SG. 2005. NIST Chemistry Webbook. Standard reference database $n^{\circ} 69$. Gaithersburg (http//webbook.nist.gov/chemistry/).

Irikura KK. 1999. Extracting thermochemical information from ab initio data. In: Minas da Piedade ME, editor. Energetics of stable molecules and reactive intermediates. Kluwer Academic Publishers, Dordrecht. pp. 353-372.

Irikura KK, Johnson RD III, Kacker RN. 2005. Uncertainties in scaling factors for ab initio vibrational frequencies. J Phys Chem A 109:84308437.

Jablonsky M, Kaszmarek A, Sadlej AJ. 2006. Estimate of the energy of intramolecular hydrogen bonds. J Phys Chem A 110:10890-10898.

Jeffrey GA. 1997. An introduction to hydrogen bonding. New York: Oxford University Press.

Jeffrey GA, Saenger W. 1994. Hydrogen bonding in biological structures. Berlin: Springer-Verlag.

Jenssen JH, Gordon MS. 1995. On the number of water molecules necessary to stabilize the glycine zwitterion. J Am Chem Soc 117:8159-8170.

Julian RR, Hodyss R, Beauchamp JL. 2001. Salt bridge stabilization of charged zwitterionic arginine aggregates in the gas-phase. J Am Chem Soc 123:3577-3583.

Kabli S, van Beelen ESE, Ingemann S, Henriksen L, Hammerum S. 2006. The proton affinities of saturated and unsaturated heterocyclic molecules. Int J Mass Spectrom 249-250:370-378.

Kebarle P. 1988. Pulsed electron high pressure mass spectrometer. In: Farrar JM, Saunders W, editors. Techniques for the study of ion-molecule reactions. New York: John Wiley \& Sons. pp. 221-286.

Kebarle P. 2003. Ion-molecule equilibria at high pressure. In: Gross ML, Caprioli RM, Armentrout P (editors). Encyclopedia of mass spectrometry, Vol. 1. Amsterdam: Elsevier. pp. 319-338.

Kebarle P, Chowdhury S. 1987. Electron affinities and electron transfer reactions. Chem Rev 87:513-534.

Kellersberger KA, Dearden DV. 2003. Low-pressure ion-molecule equilibrium. In: Gross ML, Caprioli RM, Armentrout P (editors). Encyclopedia of mass spectrometry, Vol. 1. Amsterdam: Elsevier. pp. 338-345.

Koch W, Liu B. 1989. Definitive characterization of the $\mathrm{C}_{3} \mathrm{H}_{7}{ }^{+}$potential energy surface. J Am Chem Soc 111:3479-3480.

Krug RR, Hunter WG, Grieger RA. 1976. Enthalpy-entropy compensation. 1. Some fundamental statistical problems associated with the analysis of van't Hoff and Arrhenius data. J Phys Chem 80:2335-2341.

Kuck D. 1990a. Mass spectrometry of alkyl benzenes and related compounds Part I. Mass Spectrom Rev 9:187-233.

Kuck D. 1990b. Mass spectrometry of alkyl benzenes and related compounds Part II. Mass Spectrom Rev 9:583-630.

Kuck D. 2005. Tropylium and related ions. In: Gross ML, Caprioli RM, Nibbering NMM (editors). The encyclopedia of mass spectrometry. Vol 4. Amsterdam: Elsevier.

Larson JW, McMahon TB. 1982. Formation, thermochemistry, and relative stabilities of proton-bound dimers of oxygen $n$-donor bases from ion cyclotron resonance solvent-exchange equilibrium measurements. J Am Chem Soc 104:6255-6261.

Larson JW, McMahon TB. 1985. Fluoride and chloride affinities of main groups oxides, fluorides, oxofluorides and alkyls. Quantitative scales of Lewis acidities from ion cyclotron resonance halide exchange equilibria. J Am Chem Soc 107:766-773.

Laskin J, Futrell JH. 2000. The theoretical basis of the kinetic method from the point of view of finite heat bath. J Phys Chem A 104:8829-8837.

Legon AC, Millen DJ. 1987. Directional character, strength and nature of the hydrogen bond in gas-phase dimmers. Acc chem Res 20:39-46. 
Lias SG, Bartmess JE. 2000. NIST Chemistry WebBook, NIST standard reference database number 69, Mallard WG, Linstrom PJ (Eds.) http// webbook.nist.gov.

Lias SG, Liebman JF, Levin RD. 1984. Evaluated gas phase basicities and proton affinities of molecules; heats of formation of protonated molecules. J Phys Chem Ref Data 13:695-808.

Lias SG, Bartmess JE, Liebman JF, Holmes JL, Levin RD, Mallard WG. 1988. Gas phase ion and neutral thermochemistry. J Phys Chem Ref Data 17 (Suppl. 1).

Lindh R, Roos BO, Kraemer WP. 1987. A CAS SCF CI study of the hydrogen migration potential in protonated acetylene $\mathrm{C}_{2} \mathrm{H}_{3}{ }^{+}$. Chem Phys Lett 139:407-416

Ling Y, Milburn RK, Hopkinson AC, Bohme DK. 1999. Experimental and theoretical studies of the basicity and proton affinity of $\mathrm{SiF}_{4}$ and the structure of $\mathrm{SiF}_{4} \mathrm{H}^{+}$. J Am Soc Mass Spectrom 10:848-855.

Liu L, Guo QX. 2001. Isokinetic relationship, isoequilibrium relationship and enthalpy-entropy compensation. Chem Rev 101:673-695.

Lynch BJ, Truhlar DG. 2003. Robust and affordable multicoefficient methods for thermochemistry and thermochemical kinetics: The MCCM/3 suite and SAC/3. J Phys Chem A 107:3898-3906.

Maksic ZB, Vianello R. 2002. Quest for the origin of basicity: Initial vs final state effects in neutral nitrogen bases. J Phys Chem A 106:419-430.

Malaspina T, Fileti EE, Riveros JM, Canuto S. 2006. Ab initio study of the isomeric equilibrium of the $\mathrm{HCN} \cdots \mathrm{H}_{2} \mathrm{O}$ and $\mathrm{H}_{2} \mathrm{O} \cdots \mathrm{HCN}$ hydrogen bonded clusters. J Phys Chem A 110:10303-10308.

Martin JML, de Oliveira G. 1999. Towards standard methods for benchmark quality ab initio thermochemistry-W1 and W2 theory. J Chem Phys 111:1843-1856.

Martin RL, Shirley DA. 1974. The relation of core-level binding energy shifts to proton affinity and Lewis basicity. J Am Chem Soc 96:5299-5304.

Mautner M. 1982. Carbon-hydrogen bond dissociation energies in alkylbenzenes. Proton affinities of the radicals and the absolute proton affinity scale. J Am Chem Soc 104:5-10.

Mautner M. 1984. The ionic hydrogen bond and ion solvation. 1. $\mathrm{NH}^{+} \ldots \mathrm{O}$, $\mathrm{NH}^{+} \ldots \mathrm{N}$, and $\mathrm{OH}^{+} \ldots \mathrm{O}$ bonds. Correlations with proton affinity. Deviations due to structural effects. J Am Chem Soc 106:1257-1264.

Mautner M. 2003. The proton affinity scale, and effects of ion structure and solvation. Int J Mass Spectrom 227:525-554.

Mautner M. 2005. The ionic hydrogen bond. Chem Rev 105:213-284.

Mautner M, Sieck LW. 1985. The ionic hydrogen bond and ion solvation. 4 $\mathrm{SH}^{+} \ldots \mathrm{O}$ and $\mathrm{NH}^{+} \ldots \mathrm{S}$ bonds. The correlations with proton affinity. Mutual effects of weak and strong ligands in mixed clusters. J Phys Chem 89:5222-5225.

Mautner M, Sieck LW. 1991a. Proton affinity ladders from variable temperature equilibrium measurements. 1. A reevaluation of the upper proton affinity range. J Am Chem Soc 113:4448-4460.

Mautner M, Sieck LW. 1991b. Equilibrium studies by pulsed high pressure mass spectrometry: A calibration and some pitfalls and solutions. Int $\mathrm{J}$ Mass Spectrom Ion Proc 109:187-208.

Mautner M, Hamlet P, Hunter EP, Field FH. 1980. Internal and external solvation of polyfunctional ions. J Am Chem Soc 102:6393-6399.

Mayer PM. 1999. Unimolecular reactions of proton bound cluster ions: Competition between dissociation and isomerization in the methanolacetonitrile dimer. J Phys Chem A 103:3687-3692.

Mayer PM, Glukhovstev MN, Gauld JW, Radom L. 1997. The effects of protonation on the structure, stability and thermochemistry of carboncentered organic radicals. J Am Chem Soc 119:12889-12895.

McClurg RB, Flagan RC, Goddard WA III. 1997. the hindered rotor density of states interpolation function. J Chem Phys 106:6675-6680.

McIver RT, Jr. 1970. A trapped ion analyzer cell for ion cyclotron resonance spectroscopy. Rev Sci Instrum 41:555-570.

McLuckey SA, Cameron D, Cooks RG. 1981. Proton affinity from dissociations of proton bound dimers. J Am Chem Soc 103:1313-1317.
McMahon TB. 1999. High pressure mass spectrometry. In: Minas da Piedade ME, editor. Energetics of stable molecules and reactive intermediates. Dordrecht: Kluwer Academic Publishers. pp. 259-280.

McMahon TB, Kebarle P. 1985. Bidging the gap. A continuous scale of gasphase basicities from methane to water from pulsed electron beam high pressure mass spectrometry equilibria. J Am Chem Soc 107:26122617.

McQuarrie DA. 1973. Statistical thermodynamics. New York: Harper \& Row Publishers.

Miller KJ. 1990. Additivity methods in molecular polarizability. J Am Chem Soc 112:8533-8542.

Mills BE, Martin RL, Shirley DA. 1976. Further studies of the core binding energy-proton affinity correlation in molecules. J Am Chem Soc 98:2380-2385.

Mo O, Yanez M, Decouzon M, Gal JF, Maria PC, Guillemin J-C. 1999. Gasphase basicity and acidity trends in $\alpha, \beta$-unsaturated amines phosphines and arsine. J Am Chem Soc 121:4653-4663.

Mo O, Yanez M, Eckert-Maksic M, Maksic ZB, Alkorta I, Elguero J. 2005. Periodic trends in bond dissociation energies. A theoretical study. J Phys Chem 109:4359-4365.

Mormann M, Salpin JY, Kuck D. 1999. The gas phase basicities of 6methylfulvene and 6,6-dimethylfulvene as determined by the thermokinetic method. Eur J Mass Spectrom 5:441-447.

Mormann M, Bashir S, Derrick PJ, Kuck D. 2000. Gas-phase basicities of the isomeric dihydroxybenzoic acids and gas-phase acidities of their radical cations. J Am Soc Mass Spectrom 11:544-552.

Morpurgo S, Brahimi M, Bossa M, Morpurgo GO. 2000. A theoretical study on proton transfer in the mutarotation of sugars. Phys Chem Chem Phys 2:2707-2713.

Nagaoka M, Okuyama-Yoshida N, Yamabe T. 1998. Origin of the transition state on the free energy surface: Intramolecular proton transfer reaction of glycine in aqueous solution. J Phys Chem A 102:8202-8208.

$\mathrm{Ng}$ CY, Baer T, Powis I. 1993. Cluster ions. New York: Wiley.

Nguyen VQ, Turecek F. 1997. Gas-phase protonation of pyridine. A variable time neutralization-reionization and ab-initio study of pyridinium radicals. J Mass Spectrom 32:55-63.

Nicolaides A, Rauk A, Glukhovtsev MN, Radom L. 1996. Heats of formation from G2, G2(MP2) and G2(MP2,SVP) total energies. J Phys Chem 100:17460-17464.

Nielsen SB, Bojesen G. 1995. A kinetic analysis of collision experiments in triple quadrupole instruments. Validation of the kinetic method. Eur J Mass Spectrom 1:423-428.

Norrman K, McMahon TB. 1998. Relationship between effective temperature of thermalized ions and ion source temperature. Int $\mathrm{J}$ Mass Spectrom 176:87-97.

Nourse BD, Cooks RG. 1991. Proton affinity determinations using the kinetic method in an ion trap mass spectrometer. Int J Mass Spectrom Ion Proc 106:249-272.

Ochran RA, Mayer PM. 2001. Ion rearrangement at the beginning of cluster formation: Methyl substitution effects on the internal SN2 reaction in the proton bound dimers of acetonitrile and alcohols. Eur J Mass Spectrom 7:267-277.

Ochran RA, Annamali A, Mayer PM. 2000. Unimolecular reactions of proton bound cluster ions: Competition between dissociation and isomerization in the ethanol-acetonitrile dimers. J Phys Chem A 104:85058511.

Ochterski JW, Petersson GA, Montgomery JA, Jr. 1996. A complete basis set model chemistry. V. Extensions to six or more heavy atoms. J Chem Phys 104:2598-2619.

Paizs B, Suhai S. 2005. Fragmentation pathways of protonated peptides. Mass Spectrom Rev 24:508-548.

Parthiban S, Martin JML. 2001. Assessment of W1 and W2 theories for the computation of electron affinities, ionization potentials, 
heats of formation and proton affinities. J Chem Phys 114:60146029.

Pauling L. 1960. The nature of chemical bond. Ithaca: Cornell University Press.

Pepi F, Ricci A, Rosi M. 2003. Gas phase chemistry of NHxCly+ ions; 3. Structure, stability and reactivity of protonated trichloramine. J Phys Chem A 107:2085-2092.

Petrie S. 2005. $\mathrm{CH}_{2} \mathrm{CHOH}_{2}{ }^{+}+\mathrm{PN}$ : A proton transfer triple play. J Phys Chem A 109:6326-6334.

Pham-Tran NN, Bouchoux G, Delaere D, Nguyen MT. 2005. Theoretical and experimental reevaluation of the basicity of $\lambda_{3}$-phosphinine. J Phys Chem 109:2957-2963.

Pitzer KS, Gwinn W. 1942. Energy levels and thermodynamic functions for molecules with internal rotation. J Chem Phys 10:428-440.

Poon C, Mayer PM. 2006. Competing rearrangement reactions in small pasphase ionic complexes. The internal SN2 and nitro-nitrite rearrangements in nitroalkane proton-bound pairs. Int J Mass Spectrom 255256:93-101.

Range K, Silva-Lopez C, Moser A, York DM. 2006. Multilevel and density functional electronic structure calculations of proton affinities and gasphase basicities involved in biological phosphoryl transfer. J Phys Chem A 110:791-797.

Rao JS, Sastry GN. 2006. Proton affinity of five-membered heterocyclic amines: Assessment of computational procedures. Int J Quant Chem 106:1217-1224

Raspopov SA, McMahon TB. 2005. A high-pressure mass spectrometric and density functional theory investigation of the thermochemical properties and structure of protonated dimmers and trimers of glycine. J Mass Spectrom 40:1536-1545.

Ruscic B, Berkowitz J, Pople LA. 1989. The ethyl radical: Photoionization and theoretical studies. J Chem Phys 91:114-121.

Russo N, Toscano M, Grand A, Mineva T. 2000. Proton affinity and protonation site of aniline. Energetic behavior and density functional reactivity indices. J Phys Chem 104:4017-4021.

Salpin JY, Mormann M, Tortajada J, Nguyen MT, Kuck D. 2003. The gasphase basicity and proton affinity of 1,3,5-cycloheptatriene. Energetics, structure and interconversion of dihydrotropylium ion. Eur J Mass Spectrom 9:361-376.

Scheiner S. 1985. Theoretical studies of proton transfers. Acc Chem Res $18: 174-180$

Scheiner S. 1997. Hydrogen bonding. A theoretical perspective. New York: Oxford University Press.

Schröeder D, Loos J, Schwarz H, Thissen R, Dutit O. 2004. Protonated benzene: A case for structural memory effects? J Phys Chem A 108:9931-9931.

Scott AP, Radom L. 1996. Harmonic vibrational frequencies: An evaluation of Hartree-Fock, Moller-Plesset, quadratic configuration interaction, density functional theory and semiempirical scale factors. J Phys Chem 100:16502-16513.

Sebbar N, Bockhorn H, Bozzelli JW. 2005. Thermochemical properties, rotation barriers and group additivity for unsaturated oxygenated hydrocarbons and radicals resulting from reaction of vinyl and phenyl radical systems with $\mathrm{O}_{2}$. J Phys Chem A 109:2233-42253.

Sieber S, Buezk P, Schleyer PvR, Koch W, Walkimar deCarneiro J. 1993. The $\mathrm{C}_{4} \mathrm{H}_{9}{ }^{+}$potential energy surface. J Am Chem Soc 115:259-270.

Sieck LW. 1997. Gas-phase proton affinity and protonation entropy scale between acetaldehyde and methyl acetate. An experimental evaluation and comparative analysis. J Phys Chem A 101:8140-8145.

Slaughter AR, Banna MS. 1988. Core-photoelectron binding energies of gaseous glycine: Correlation with its proton affinity and gas phase acidity. J Phys Chem 92:2165-2167.

Smith BJ, Radom L. 1992. Ethylamine: The remarkable acid strengthening and base weakening effect of the acetylenic linkage. A comparison with ethylamine and methylamine. J Am Chem Soc 114:36-41.
Smith BJ, Radom L. 1993. Assigning absolute value to proton affinities: A differentiation between competing scales. J Am Chem Soc 115:48854888.

Smith BJ, Radom L. 1994. An evaluation of the performance of density functional theory, MP2, MP4, F4, G2(MP2) and G2 procedures in predicting gas phase proton affinities. Chem Phys Lett 231:345-351.

Smith BJ, Radom L. 1995. Calculation of proton affinities using the G2(MP2,SVP) procedure. J Phys Chem 99:6468-6471.

Sobczyk L, Grabowski SJ, Krygowski TM. 2005. Interrelation between H-bond and pi-electron delocalisation. Chem Rev 105:3515-3560.

Speller CV, Mautner M. 1985. The ionic hydrogen bond and ion solvation. 3. Bonds involving cyanides. Correlations with proton affinities. J Phys Chem 89:5217-5222.

Splitter JS, Turecek F. 1994. Applications of mass spectrometry to organic stereochemistry. New York: VCH Publishers.

Stace AJ. 1995. Techniques for the preparation and study of cluster ions. In: Jenning K, editor. Fundamental and applications of gas phase ion chemistry. Dordrecht: Kluwer Academic Publisher. pp. 381-400.

Staley RH, Beauchamp JL. 1974. Relationship of nitrogen lone pair interactions to thermodynamic parameters associated with amine basicities. J Am Chem Soc 96:1604-1606.

Staley RH, Kleckner JE, Beauchamp JL. 1976. Relationship between orbital ionisation energies and molecular properties. Proton affinities and photoelectron spectra of nitrile. J Am Chem Soc 98:2081-2085.

Steinfeld JI, Francisco JS, Hase WL. 1989. Chemical kinetics and dynamics. Englewood Cliffs: Prentice-Hall.

Swart M, Rösler E, Bickelhaupt FM. 2006. Proton affinities of maingroupelements hydrides and noble gases: Trends across the periodic table, structural effects and DFT validation. J Comput Chem 27:1486-1493.

Szulejko JE, McMahon TB. 1993. Progress toward an absolute gas-phase proton affinity scale. J Am Chem Soc 115:7839-7848.

Szymczak JJ, Urban J, Roszak S, Leszczynski J. 2006. The nature of variations of ammonia proton affinity in an Argon environment. J Phys Chem 110:13099-13105.

Taft RW, Topsom RD. 1987. The nature of substituent electronic effects. Prog Phys Org Chem 16:1-83.

Tasi G, Mizukami F, Palinko I, Csontos J, Gyorffy W, Nair P, Maeda K, Toba M, Niwa S, Kiyozumi Y, Kiricsi I. 1998. Enumeration of the conformers of unbranched aliphatic alkanes. J Phys Chem 102:7698-7703.

Truhlar DG. 1991. A simple approximation for the vibrational partition function of a hindered internal rotation. J Comput Chem 12:266270.

Uggerud E. 1992. Properties and reactions of protonated molecules in the gas phase. Experiment and theory. Mass Spectrom Rev 11:389-430.

van Beelen ESE, Koblenz TA, Ingemann S, Hammerum S. 2004. Experimental and theoretical evaluation of proton affinities of furan, the methylphenols and the related anisoles. J Phys Chem A 108:27872793.

van der Rest G, Mourgues P, Leblanc D, Audier HE. 1997. Reactions of the allyl and 2-propenyl cations isomers with D2O: Separation and titration of mixed isomers by Fourier transform ion cyclotron resonance. Eur Mass Spectrom 3:323-326.

Van Speybroeck V, Van Neck D, Waroquier M, Wauters S, Sayes M, Marin GB. 2000. Ab initio study of radical addition reactions: Addition of a primary ethylbenzene radical to ethane. J Phys Chem A 104:1093910950.

Van Speybroeck V, Borremans Y, Van Neck D, Waroquier M, Wauters S, Sayes M, Marin GB. 2001. Ab initio study of radical reactions: Cyclisation pathways for the butylbenszene radical. J Phys Chem A 105:7713-7723

Van Speybroeck V, Van Neck D, Waroquier M. 2002. Ab initio study of radical reactions: Role of coupled internal rotations on the reaction kinetics. J Phys Chem A 106:8945-8950. 
Van Speybroeck V, Vansteenkiste P, Van Neck D, Waroquier M. 2005. Why does the uncoupled hindered rotor model work well for the thermodynamics of $n$-alkanes? Chem Phys Lett 402:479-484.

Vansteenkiste P, Van Speybroeck V, Marin GB, Waroquier M. 2003. Ab initio calculation of entropy and heat capacity of gas-phase $n$-alkanes using internal rotations. J Phys Chem A 107:3139-3145.

Vansteenkiste P, Van Speybroeck V, Verniest G, De Kimpe N, Waroquier M. 2006. Applicability of the hindered rotor scheme to the puckering mode in four membered rings. J Phys Chem A 110:3838-3844.

Vekey K. 1996. Internal energy effects in mass spectrometry. J Mass Spectrom 31:445-463.

Wang Z, Chu IK, Rodriquez CF, Hopkinson AC, Siu KWM. 1999. $\alpha, \omega-$ Diaminoalkanes as models for bases that dicoordinate the proton: An evaluation of the kinetic method for estimating their proton affinities. J Phys Chem 103:8700-8705.

Wesdemiotis C. 2004. Entropy considerations in kinetic method experiments. J Mass Spectrom 39:998-1003.

Wetmore SD, Schofield R, Smith DM, Radom L. 2001. A theoretical investigation of the effects of electronegative substitution on the strength of C-H $\cdots \mathrm{N}$ hydrogen bonds. J Phys Chem A 105:8718-8726.

Witschel W, Hartwigsen C. 1997. Partition function and the level density of the hindered rotor. Chem Phys Lett 273:304-310.

Witt M, Grutzmacher HF. 1997. Proton bond dimers of aliphatic carboxamides: Gas phase basicity and dissociation energy. Int J Mass Spectrom Ion Proc 165/166:49-62.

Wolf JF, Staley RH, Koppel I, Taagepera M, McIver RT, Jr, Beauchamp JL, Taft RW. 1977. Gas phase basicities and relative proton affinities of compounds between water and ammonia from pulsed ion cyclotron resonance thermal equilibria measurements. J Am Chem Soc 99: $5417-5429$

Wu Z, Fenselau C. 1994. Gas phase basicities and proton affinities of lysine and histidine measured from the dissociation of proton bound dimers. Rapid Commun Mass Spectrom 8:777-780.

Wu J, Lebrilla CB. 1993. Gas phase basicities and sites of protonation of glycine oligomers $\left(\mathrm{GLY}_{n} ; n=1-5\right)$. J Am Chem Soc 115:3270-3275.

Wu R, McMahon TB. 2005. An investigation of the ion-molecule interactions of protonated glycine with ammonia by high pressure mass spectrometry and ab initio calculations. Can J Chem 83:1978-1993.

Yanez M. 2003. Energies and potential energy surfaces: Organics. In: Gross ML, Caprioli RM, Armentrout P (editors). Encyclopedia of mass spectrometry, Vol. 1. Amsterdam: Elsevier. pp. 68-77.

Zhang K, Chung-Phillips A. 1998. Conformers of gaseous protonated glycine. J Comput Chem 19:1862-1876.

Zhang K, Zimmerman DM, Chung-Philips A, Cassady CJ. 1993. Experimental and ab initio studies of the gas phase basicities of polyglycines. J Am Chem Soc 115:10812-10822.

Zheng X, Cooks RG. 2002. Thermochemical determination by the kinetic method with direct entropy correction. J Phys Chem A 106:9939-9946.

Zheng X, Cooks RG, Augusti R, Tao WA. 2003. The kinetic method: Thermochemical determinations and chiral analysis. In: Gross ML, Caprioli RM, Armentrout P (editors). Encyclopedia of mass spectrometry, Vol. 1. Amsterdam: Elsevier. pp. 350-362.

Zhu L, Bozzelli JW. 2002. Structures, rotational barriers and thermochemical properties of chlorinated aldehydes and the corresponding acetyl $(\mathrm{CC} .=\mathrm{O})$ and formyl methyl $(\mathrm{C} . \mathrm{C}=\mathrm{O})$ and additivity groups. J Phys Chem A 106:345-355.

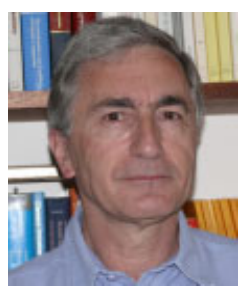

Guy Bouchoux was born in Paris in 1946. He studied chemistry at Orsay University (France) where he is teaching since 1970 and where he is presently a Professor of chemistry. During the same period, he was conducting his research at the Ecole Polytechnique (Palaiseau). His research interests include gas-phase ion chemistry (unimolecular and ion-molecule reactions of cations and radical cations), thermochemistry (protonation energetics, quantum chemical calculations) and mass spectrometry. 